\title{
Werner Kogge
}

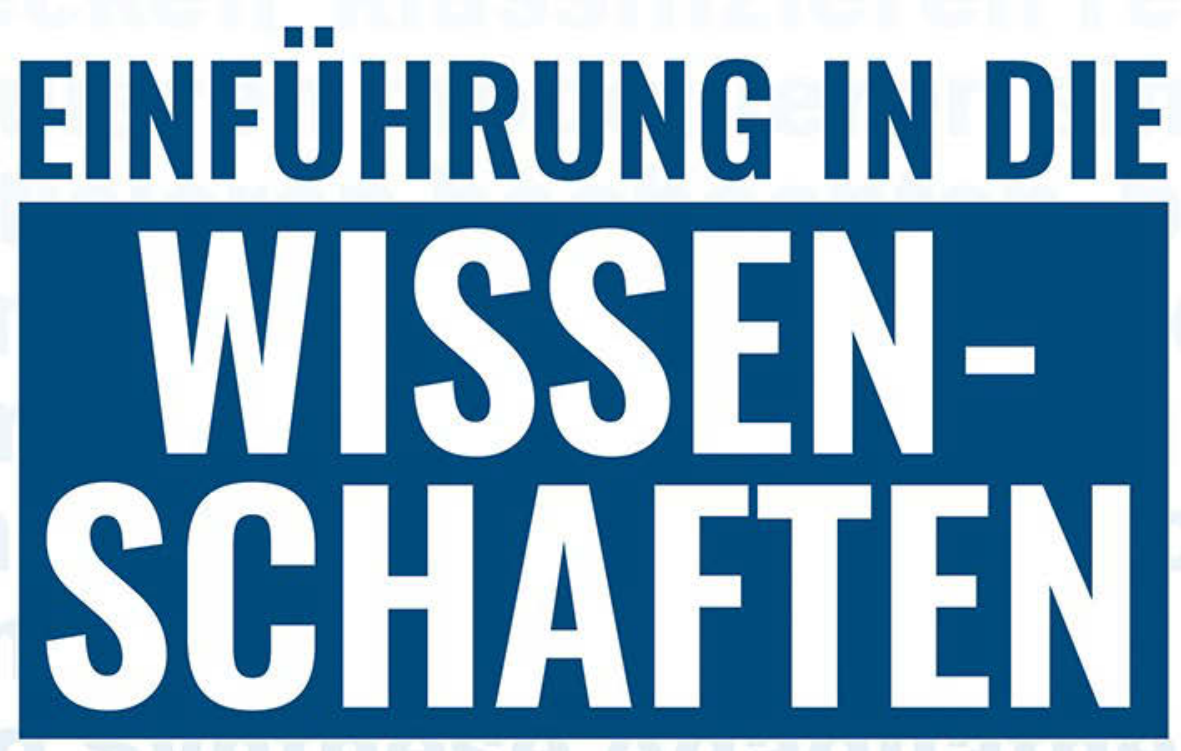

\section{Wissenschaftstypen \\ Deutungskämpfe \\ Interdisziplinäre Kooperation}

[transcript] sciencestudies 
Werner Kogge

Einführung in die Wissenschaften

Science Studies 
Werner Kogge lehrt Philosophie an der Freien Universität Berlin und leitet den Bereich Wissenschaftsphilosophie und Politische Theorie der DFG-Kollegforschungsgruppe "Strategies of Governance and Modes of Participation in the Ancient Near East«. Seine Forschungsschwerpunkte liegen im Feld von Methodologie, Wissenschaftstheorie, Politischer Theorie und Begriffsforschung. 
Werner Kogge

\section{Einführung in die Wissenschaften}

Wissenschaftstypen - Deutungskämpfe - Interdisziplinäre Kooperation 
Die Publikation wurde ermöglicht durch eine Ko-Finanzierung für Open-AccessMonografien und -Sammelbände der Freien Universität Berlin.

\section{Bibliografische Information der Deutschen Nationalbibliothek}

Die Deutsche Nationalbibliothek verzeichnet diese Publikation in der Deutschen Nationalbibliografie; detaillierte bibliografische Daten sind im Internet über http://dnb.d-nb.de abrufbar.

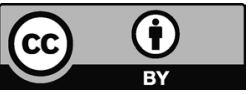

Dieses Werk ist lizenziert unter der Creative Commons Attribution 4.o Lizenz (BY). Diese Lizenz erlaubt unter Voraussetzung der Namensnennung des Urhebers die Bearbeitung, Vervielfältigung und Verbreitung des Materials in jedem Format oder Medium für beliebige Zwecke, auch kommerziell. (Lizenztext:

https://creativecommons.org/licenses/by/4.o/deed.de)

Die Bedingungen der Creative-Commons-Lizenz gelten nur für Originalmaterial. Die Wiederverwendung von Material aus anderen Quellen (gekennzeichnet mit Quellenangabe) wie z.B. Schaubilder, Abbildungen, Fotos und Textauszüge erfordert ggf. weitere Nutzungsgenehmigungen durch den jeweiligen Rechteinhaber.

\section{Erschienen 2022 im transcript Verlag, Bielefeld (c) Werner Kogge}

Umschlaggestaltung: Kordula Röckenhaus, Bielefeld Korrektorat: Jonas Geske, Bielefeld Druck: Majuskel Medienproduktion $\mathrm{GmbH}$, Wetzlar

Print-ISBN 978-3-8376-5970-2

PDF-ISBN 978-3-8394-5970-6

https://doi.org/10.14361/9783839459706

Buchreihen-ISSN: 2703-1543

Buchreihen-eISSN: 2703-1551

Gedruckt auf alterungsbeständigem Papier mit chlorfrei gebleichtem Zellstoff. Besuchen Sie uns im Internet: https://www.transcript-verlag.de Unsere aktuelle Vorschau finden Sie unter www.transcript-verlag.de/vorschau-download 


\section{Inhalt}

Vorwort. 9

Einleitung: Die Wissenschaft und die Wissenschaften 13

I. Drei Traditionen von Wissenschaft und ihre Quellen . 17

1. Quellen moderner Wissenschaften I: Die Traditionslinie Episteme-Scientia - Der Wendepunkt im 12. Jahrhundert ................... 17

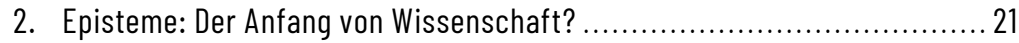

BASISWISSEN 1: Episteme im Kontext............................................ 22

3. Die Verallgemeinerung der Episteme: Wissenschaft als Beweisen.......... 24

4. Deutungskämpfe I: Naturwissenschaften versus Geisteswissenschaften - Erklären gegen Verstehen................. 27

5. Quellen moderner Wissenschaften II: Die Tradition von Hermeneutik

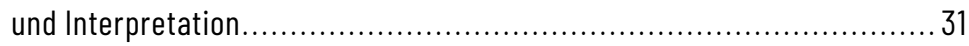

6. Verstehen als Arbeit an sich selbst und den eigenen Konzepten ............ 32 BASISWISSEN 2: Hermeneutik - Verstehende Wissenschaft .................. 33

7. Quellen moderner Wissenschaften III: Die Tradition der Naturalis historia - Beschreiben, Entdecken und Klassifizieren ...................... 36

BASISWISSEN 3: Historia - ein missverständliches Wort .................... 37

II. Auffächerungen in den drei Wissenschaftstraditionen ............... 41

8. Noch einmal zum Beweisen: Die Rolle der Mathematisierung ................. 41

BASISWISSEN 4: Axiome - Grundlagen von Schlussfolgerungen ............ 42

VERTIEFEND: Mathematik versus Logik ..................................... 45

BASISWISSEN 5: Beweise - Zum Verhältnis von Logik und Mathematik ..... 46

9. Die Rolle des Experimentierens und das Schicksal der Induktion .......... 52 BASISWISSEN 6: Induktion und Empirie - der Weg der Erfahrung ............ 56 
10. Laborwissenschaft, Modellierung, Simulation und 'Realexperimente $<$...... 60

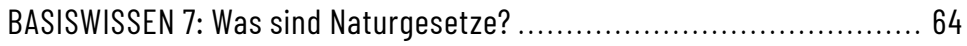

VERTIEFEND: Symbolismen in den Wissenschaften ......................... 67

11. Noch einmal zum Verstehen: Das Forschungsparadigma der Hermeneutik - Was haben Verstehenslehren mit Kriminalistik zu tun? Der Ansatz der Rekonstruktion .................... 69 BASISWISSEN 8: Analyse und Synthese ein Begriffspaar fährt Achterbahn .............................................. 72

12. Adäquatheit - Von der Anpassung der Konzepte

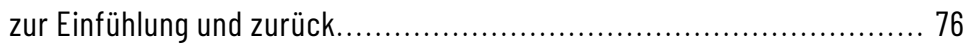

VERTIEFEND: Zur Begriffsgeschichte von Objektivität ....................... 80

BASISWISSEN 9: Was bedeutet Objektivität? ................................ 84

13. Der 'Sehepunkt des Verstehens - das Paradigma der Kritik: Wissenschaftlichkeit durch Reflexivität .................................... 86

14. Noch einmal zum Beschreiben: Das Wissenschaftsideal der Deskription Beobachten, Beschreiben, Tatsachen ......................................... 92

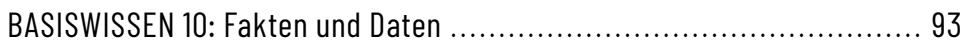

VERTIEFEND: Empirie im Empirismus und Positivismus ....................... 96

15. Wissenschaft und die Ordnung der Dinge: Relationen, Strukturen und die Praktiken des Vergleichens und Typisierens

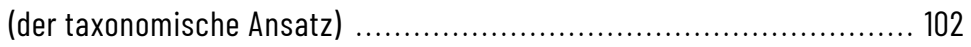
BASISWISSEN 11: Erscheinung und Wirklichkeit die Suche nach einer verborgenen Ordnung..................................... 104 VERTIEFEND: Probleme im Vergleichen ......................................... 106 VERTIEFEND: Strukturalismus und die Ordnung der Differenzen ............ 107

16. Die Rolle der Statistik: Daten, Mengen, Regelmäßigkeiten und eine Irrfahrt ins Reich der Wahrscheinlichkeit ........................... 111

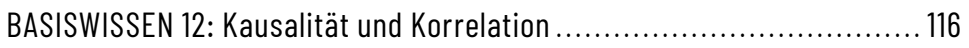
VERTIEFEND: Der (Irr-)Weg der Statistik ................................................... 119

III. Die Pluralität der Wissenschaften: Reflexionen ....................... 131

17. Einteilungen von Wissenschaften I: Frühe Abgrenzungen, Kants Systematisierung und die Bipolarisierung von Geistesund Naturwissenschaften im 19. Jahrhundert ......

18. Deutungskämpfe II: Kontroversen im 20. Jahrhundert:

Einheitswissenschaften, Denkstile, Paradigmen und die Rolle der Wissenschafts- und Technikforschung (STS) 
BASISWISSEN 13: Konstruktivismus -

ein Schlagwort, unterschiedliche Bedeutungen .............................. 154

VERTIEFEND: Spielarten des Konstruktivismus............................... 155

VERTIEFEND: Wissenschaftliches Wissen

in der Wissenschaftssoziologie

159

19. Einteilungen von Wissenschaften II:

Wissenschaftskulturen werden Thema...................................... 162

IV. Wissenschaftstypen als Tiefenstruktur

interdisziplinärer Zusammenarbeit ........................................... 171

20. Typologie der Wissenschaft: Neun Wissenschaftstypen als Typen

mit Familienähnlichkeiten

21. Die Pluralität der Wissenschaften und das Problem

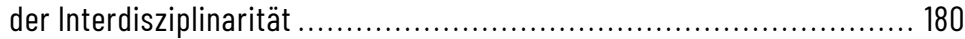

VERTIEFEND: Konzepte der Interdisziplinaritätsforschung ................... 184

22. Noch einmal zum Konzept der Wissenschaftstypen: Idealtypen mit Anschlussstellen: Ein modulares und kombinatorisches

Konzept interdisziplinärer Zusammenarbeit 190

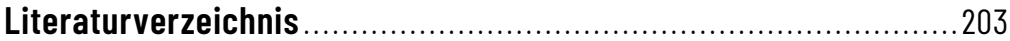

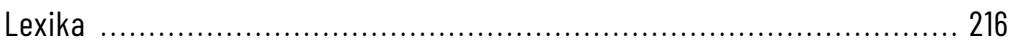

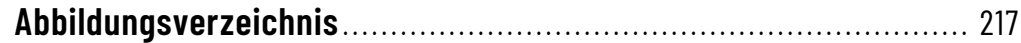

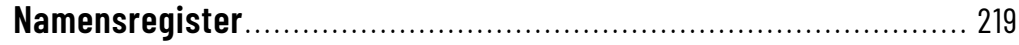

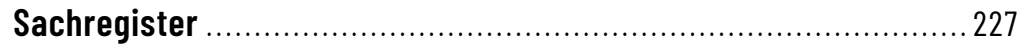





\section{Vorwort}

Pluralistische Wissenschaftstheorie ist kein etabliertes Genre. Bislang nehmen Einführungen in die Wissenschaften stets einen besonderen Blickwinkel ein. Sie erklären Wissenschaft aus der Perspektive bestimmter Vorstellungen und Ausprägungen von Wissenschaft, verbunden mit häufig nur impliziten Wertungen und unbefragten Präferenzen. Was in den Kämpfen um Deutungshoheit auf der Strecke bleibt, ist eine pluralistische und integrative Sicht auf Wissenschaft; eine Sichtweise, die von der Voraussetzung ausgeht, dass unterschiedliche Typen von Wissenschaft existieren, dass diese zunächst einmal als gleichberechtigt anzusehen sind und dass ihnen weder eine hierarchische Staffelung noch eine evolutionäre Entwicklungslinie unterlegt werden kann. Eine pluralistische Sichtweise zielt auf eine radikal egalitäre Darstellung der Wissenschaften als eigenständige Typen, mit je eigenen inneren Motiven und Praktiken.

Deutungskämpfe darum, was Wissenschaft eigentlich ist, zeigen sich in dieser Sichtweise als Resultat nicht nur von Intoleranz, sondern vor allem eines Nichtwissens darum, wie andere Typen von Wissenschaften in sich strukturiert, motiviert und validiert sind. Differenzen zwischen unterschiedlichen Wissenschaftstypen zu verstehen und zu akzeptieren, ermöglicht dagegen eine Form der interdisziplinären Kooperation, die in den Tiefenstrukturen der Wissenschaftstypen ansetzt.

Der Aufbau des Buches setzt auf eine Verknüpfung von historischer Tiefenschärfe und Entfaltung aktueller Realitäten. Im ersten Teil mit dem Titel Drei Traditionen von Wissenschaft und ihre Quellen wird nachgezeichnet, wie aus drei antiken Quellen im Übergang vom Spätmittelalter zur frühen Neuzeit sich drei Traditionen von Forschung herausbildeten, die mit unterschiedlichen Weisen $\mathrm{zu}$ fragen, $\mathrm{zu}$ suchen und $\mathrm{zu}$ antworten verbunden waren. 
Im zweiten Teil wird unter dem Titel Auffächerungen in den drei Wissenschaftstraditionen die weitere Entfaltung dieser Traditionen dargestellt und gezeigt, wie sie noch heute bestimmen, was in den akademischen Disziplinen unter Wissenschaft verstanden und als Forschung praktiziert wird. Dabei lassen sich neun Typen von Wissenschaft unterscheiden.

Im dritten Teil wird das Thema Deutungskämpfe und Klassifikation von Wissenschaften unter dem Titel Die Pluralität der Wissenschaften: Reflexionen historisch eingefangen: Klassische Auseinandersetzungen um die Bestimmung von Wissenschaft, aber auch Ansätze der Typisierung von Wissenschaften werden hier vorgestellt.

Der vierte Teil, Wissenschaftstypen als Tiefenstruktur interdisziplinärer Zusammenarbeit, entwickelt einen Neuansatz zur Interdisziplinarität. Das Problem der Interdisziplinarität kann nicht als gelöst angesehen werden. Es fehlt nach wie vor an überzeugenden Ansätzen, wie unterschiedliche disziplinäre Sicht- und Vorgehensweisen effektiv in eine kooperative Forschungsform integriert werden können. Interdisziplinarität droht deshalb von einem überzeugend motivierten Anliegen zu einer wissenschaftspolitischen Leerformel zu werden. Durch die Entfaltung der Tiefenstruktur von Wissenschaftstypen zeigt sich hier nun die Möglichkeit einer neuen Vorgehensweise: ein modulares und kombinatorisches Konzept interdisziplinärer Zusammenarbeit.

Da die Geschichte der Wissenschaftstheorie von einseitigen und kämpferisch eingesetzten Konzepten geprägt ist, stellen sich Begriffe, die Wissenschaftlichkeit kennzeichnen - wie z.B. Empirie, Fakten, Induktion, Kausalität - in der Praxis häufig als verworren und wenig konturiert heraus. In gesonderten Kästen wird in diesem Buch zu solchen Begriffen innerhalb der historischen Darstellung ein knapper Abriss von BASISWISSEN gegeben, in dem aufgezeigt wird, welche klaren Problemstellungen und Ideen einen Begriff prägten.

Weitere durch Doppelpfeile abgesetzte Kästen bieten VERTIEFUNGEN an. Das sind Textteile, die Zusammenhänge erläutern, die für die Debatten um Wissenschaftlichkeit eine Rolle spielen, aber gleichzeitig so komplex sind, dass sie den Lesefluss unterbrechen würden, wären sie in den Haupttext auf gleicher Ebene inkludiert. Diese Abschnitte können somit in der Lektüre zunächst übersprungen, aber zu jeder Zeit nachgelesen werden, wenn Bedarf an einer vertiefenden Darstellung besteht. 
Das Buch basiert auf zwei Jahrzehnten Erfahrung mit interdisziplinärer Netzwerkarbeit in unterschiedlichen Forschungsverbünden. Es zeigte sich vielfach, dass es in der Projektierung, Leitung und Zusammenarbeit in solchen Verbünden viel Zeit und Energie erfordert, überhaupt zu verstehen, wie andere Formen und Auffassungen von Wissenschaft in sich 'gestrickt s sind. Spannungen und Missverständnisse sind die Folge einer fehlenden Orientierung im vielfältigen Feld der Wissenschaften.

Für alle, die interdisziplinäre Forschung planen und leiten, gibt das Buch eine `Karte an die Hand, um die Landschaft der Wissenschaftstypen zu überblicken und bestimmte Ziele anzusteuern. Das Gleiche gilt aber auch für alle Beteiligten, nicht nur an interdisziplinärer Forschung, die daran interessiert sind, ihre eigene Forschungspraxis im Verhältnis zu anderen Formen von Wissenschaft zu verorten.

Für Einsteiger_innen, die ihren Weg in die Wissenschaften noch suchen, kann das Buch als ein Leitfaden für persönliche Weichenstellungen dienen: Welcher Typus Wissenschaft liegt mir? Wo und wie würde ich gerne arbeiten?

Darüber hinaus stellt es einem breiten Publikum die Möglichkeit zur Verfügung, sich einen Überblick über das ganze Spektrum zu verschaffen, was an Universitäten und Forschungseinrichtungen als Wissenschaft praktiziert und gesellschaftlich finanziert wird. 



\section{Einleitung: Die Wissenschaft und die Wissenschaften}

Klara G. studiert seit Stunden einen Keilschrifttext. Sie sucht nach Zeichensequenzen, die sie identifizieren kann, stellt Vermutungen über mögliche Sinnzusammenhänge und das Textgenre an, konsultiert Zeichenlisten, Wörterbücher und Interpretationen früherer Bearbeiter_innen, prüft, überdenkt, sieht ein neuen Aspekt. Peter P. testet die vierunddreißigste Mischung aus DNA-Fragmenten und Restriktionsenzymen, die er tags zuvor mittels Pipette auf einem Nährmedium aufbrachte, probiert unterschiedliche Temperaturen und Zeitspannen, doch bisher sind die Ergebnisse noch unspezifisch. Melanie N. exzerpiert einen sprachphilosophischen Text zur Bedeutungstheorie und versucht die wichtigsten Thesen und Argumente in übersichtlich gegliederten Notizen aufzuzeichnen, mit dem Ziel, an die Stelle einer unklaren Behauptung eine stichhaltige Argumentation zu setzen. David M. befragt Väter zu ihrer Beteiligung an Kindererziehung und Haushalt und trägt die Ergebnisse in ein Antwortraster ein, das er entwickelt hat. Er unterzieht die Daten einer Analyse mittels statistischer Methoden und passt mehrmals das Forschungsdesign an, um die Signifikanz der Aussagen zu stärken.

Sie alle sind in Forschungsinstituten tätig oder verfassen wissenschaftliche Qualifikationsarbeiten. Doch was verbindet ihre Tätigkeiten? Was macht diese Tätigkeiten zu Wissenschaft? Wo fängt Wissenschaft an und wo hört Wissenschaft auf?

Wissenschaftliche Disziplinen haben sich in ungeahnter Weise vervielfältigt. Studiengangsportale listen, je nach Auflösung, zurzeit 100 bis 500 Studiengänge auf. Studiengänge sind nicht Disziplinen und Disziplinen sind nicht Wissenschaften. Doch wie viele Wissenschaften gibt es? Sind Disziplinen Teile von Wissenschaften? - oder Teile der Wissenschaft? Zuweilen sieht 
es so aus, als gäbe es ein einheitliches Unternehmen mit Namen Wissenschaft. Dann wieder, als existierten nur Disziplinen, die sich unvereinbar zeigen schon darin, was unter Wissenschaft überhaupt zu verstehen sei. Wissenschaftstheorie, Wissenschaftsphilosophie und Wissenschaftssoziologie bemühen sich um Konzepte von Wissenschaft. Doch je genauer man hinsieht, desto komplizierter wird das Bild. Ein Resultat dieser Komplikation ist, dass sich keine klare Idee davon findet, worin sich wissenschaftliche Disziplinen unterscheiden und auf welchen Grundlagen und in welchen Weisen unterschiedliche Disziplinen wissenschaftlich kooperieren können.

Interdisziplinarität ist der Begriff, unter dem sich Bemühungen versammeln, die auf die Differenzierung der Disziplinen reagieren. Als Stichwort inzwischen schon etwas abgestumpft, wird Interdisziplinarität aber mit jedem neuen Forschungsverbund, mit jedem übergreifenden Kooperationsprojekt zur Aufgabe. Doch von Beginn an krankte die Idee daran, dass sich grundsätzliche Fragen als widerspenstig erwiesen: Was ist es denn, was die Disziplinen trennt und was ist es, worin sie sich verbinden könnten? Anders gesagt: Wären alle Disziplinen Teile eines einheitlichen wissenschaftlichen Systems, dann wären sie bereits integriert und ihre Integration könnte durch einfache Maßnahmen intensiviert werden. Zeigten sich Disziplinen aber als jeweils eigengesetzliche Unternehmen der Wissensproduktion, dann stellt sich die Frage, in welchem Medium oder in welchem übergreifend gültigen Regelwerk sie ihre Potenziale verknüpfen können. Es sind also zumindest zwei Beziehungen zu klären, wenn es um Kooperation und Integration wissenschaftlicher Disziplinen geht: zum einen, wie es um Einheit und Vielfalt der Wissenschaft(en) steht; zum anderen, was, im Falle von Wissenschaft(en), Kooperation überhaupt bedeuten kann.

In der Geschichte der Ideen ist sowohl die Einheit der Wissenschaft als auch deren Vielzahl immer wieder herausgestellt worden: Mal sah man das Gemeinsame der Wissenschaft in Prozeduren formaler Sprachen wie bei Descartes, Leibniz und im Logischen Empirismus, mal in vorausgesetzten Strukturen von Erkenntnis und Vernunft wie bei Kant und seinen Nachfolgern, ${ }^{1}$ mal in der Anknüpfung an kosmologische Konzepte der Naturge-

1 Vgl. hierzu: Akademie der Wissenschaften zu Berlin (Bernd Grafräth/Renate Huber/Brigitte Uhlemann): Einheit, Interdisziplinarität, Komplementarität. Orientierungsprobleme der Wissenschaft heute, Berlin/New York 1991. 
schichte wie bei Herder und Humboldt, ${ }^{2}$ mal in einer "Unity of Science,${ }^{3}$ die physikalistisch, ${ }^{4}$ hermeneutisch ${ }^{5}$ oder systemtheoretisch ${ }^{6}$ begründet wurde. Woher stammt dann aber das Problem der Vielheit? Ist dies nur ein Resultat der Einrichtung und Institutionalisierung neuer Disziplinen? »Die Antike und das Mittelalter sehen die Einheit der Wissenschaft gewährleistet in der als selbstverständlich angenommenen kosmischen bzw. göttlichen Weltordnung ${ }^{7}$ - liest man. Doch ist das wahr? Tatsächlich kamen die Philosophie-, die Ideen- und die Institutionengeschichte des Mittelalters zu anderen Ergebnissen - es sei denn, man setzt den Einschnitt zwischen Mittelalter und Neuzeit genau da, wo sich die ersten Universitäten herauszubilden beginnen: im 12. und 13. Jahrhundert. Hier nämlich entsteht der ideelle und institutionelle Komplex, der viele entscheidende Züge dessen aufweist, was sich die Moderne unter Wissenschaft vorstellt. Was traf hier aufeinander, dass eine solch weitreichende Konstellation entstehen konnte? Es ist eine Geschichte von Eroberung und kulturellem Austausch, von verschollenen und wiederentdeckten Schriften, von Verlockungen, durch Wissen Macht zu befestigen und von - bereits hier - Konkurrenzen um die für Wahrheit bürgende Form der Erkenntnis und Weltdeutung.

2 Vgl. Bernhard Sticker: ^Alexander von Humboldt und die Einheit der Wissenschaftt. Studia Leibnitiana, Bd. 2, H. 4 (1970), S. 241-261.

3 Vgl. Paul Oppenheim/Hilary Putnam: Unity of science as a working hypothesis. In: Herbert Feigl (Hrsg.): Concepts, theories and the mind-body problem. Minnesota studies in the philosophy of science. Minneapolis: University of Minnesota Press, 1958, S. 3-36.

4 Vgl. Max Planck: Die Einheit des physikalischen Weltbildes. Vortrag, gehalten am 9. Dezember 1908 in der naturwissenschaftlichen Fakultät des Studentenkorps an der Universität Leiden, Leipzig 1909.

5 Vgl. Karin Knorr-Cetina: Die Fabrikation von Erkenntnis. Zur Anthropologie der Wissenschaft, Frankfurt a.M. 1987. Darin Kapitel 7: Wissenschaft als interpretative Rationalität oder: Die Übereinstimmung zwischen den Natur- und Sozialwissenschaften, S. 245-270.

6 Akademie der Wissenschaften zu Berlin: Einheit, Interdisziplinarität, Komplementarität, 1991, S. 17-27.

7 Akademie der Wissenschaften zu Berlin: Einheit, Interdisziplinarität, Komplementarität, 1991, S. 8. 



\section{Drei Traditionen von Wissenschaft und ihre Quellen}

\section{Quellen moderner Wissenschaften I: Die Traditionslinie Episteme-Scientia - Der Wendepunkt im 12. Jahrhundert}

Im Zentrum des Paradigmenwechsels, der sich im 12. und 13. Jahrhundert vollzog, steht die Idee, dass Wissenschaft durch ein bestimmtes Verfahren gekennzeichnet sei, nämlich durch das Beweisen (lateinisch: demonstrare). Diese Idee ist - nicht nur zu dieser Zeit - alles andere als selbstverständlich, aber dafür umso folgenreicher. Warum prägte sie sich gerade in dieser Zeit aus?

Grund hierfür sind Verwicklungen in der Überlieferungs- und Übersetzungsgeschichte der aristotelischen Schriften. Aristotelische Texte wurden früh ins Lateinische übersetzt und das Mittelalter hindurch kontinuierlich tradiert. Dies gilt auch für die logischen Schriften aus dem sogenannten Organon - mit einer Ausnahme: die Analytica Posteriora (Zweite Analytik), die auch unter dem Titel Lehre vom Beweis geführt werden. Zwei Punkte sind hier für die Rezeption und Umsetzung in ein neues Wissenschaftskonzept entscheidend: Zum einen fand sich in diesem Werk ein Bild von Wissenschaft als einem zusammenhängendem Feld, das sich in verschiedene Bereiche unterteilt; zum anderen war dieses Vorgehen dadurch gekennzeichnet, dass es notwendige Zusammenhänge aufzeigt, also Beziehungen, für die es unmöglich ist, dass es sich anders verhält. ${ }^{1}$ Wissenschaft als eine Erkenntnisform, die sämtliche Wissensbereiche durchdringt und auf zwangsläufige

1 Vgl. Otfried Höffe: Einführung in die Wissenschaftstheorie der Zweiten Analytik. In: Aristoteles: Lehre vom Beweis oder Zweiten Analytik (Organon IV), übers.u. m. Anm. vers. v. Eugen Rolfes; m. neuer Einl. u. Bibliographie v. Otfried Höffe, Hamburg 1990, S. VII-XLII, hier XIXff. 
Weise Wahrheiten zutage fördert - das Potenzial, das hier schlummerte, liegt auf der Hand.

Dieses Verständnis einer ebenso allumfassenden wie zwingenden Vorgehensweise prägte eine wirkungsgeschichtlich mächtige und bis heute zentrale Auffassung von Wissenschaft. Wir werden noch sehen, dass diese Auffassung, trotz aller Selbstverständlichkeit, die sie in sich versammelt, keineswegs konkurrenzlos ist und dass sie auch keineswegs als eine alternativlose Deutung der aristotelischen Erkenntnislehre gelten kann. Wir werden sehen, dass der Begriff, um den sich hier alles dreht, bei Aristoteles eine ganz besondere Bedeutung hatte und eine besondere Wissensform bezeichnete. Episteme - dies ist das griechische Wort, das in den lateinischen Übersetzungen mit scientia, in modernen auch mit Wissenschaft wiedergegeben wird, ist alles andere als ein problemloser Vorläufer moderner Vorstellungen. Dazu kommt, dass Aristoteles eine Reihe anderer Erkenntnisformen kennt, die - noch bis ins 12. Jahrhundert - gleichberechtigt neben der Erkenntnisform episteme stehen, wie etwa Klugheit (phronesis) und Könnerschaft (techné). ${ }^{2}$ Letzteres, die Fertigkeit im Herbeiführen oder Herstellen, ist im lateinischen Mittelalter in ihrer Übersetzung als Kunst (ars) die Bezeichnung für das Bildungsgut, das es zu erwerben und vermitteln galt. Die Septem Artes, die Sieben freien Künste (Grammatik, Rhetorik. Dialektik, Arithmetik, Geometrie, Musik und Astronomie), gliederten die Ausbildung in den Schulen und Hochschulen, wohl geeint unter dem Dach einer sie umfassenden Theologie. ${ }^{3}$

Der wohlsituierte Status der aristotelischen episteme im Kontext eines differenzierten Feldes von Erkenntnis- und Wissensformen geriet erst ins Wanken, als sich im 12. und 13. Jahrhundert auch auf anderem Weg eine aristotelische Wissenschaftskonzeption im christlichen Abendland durchzusetzen begann. Die kastilische Stadt Toledo, 1085 erobert von den Mauren, entwickelte sich zu dieser Zeit zu einem intellektuellen Zentrum Europas. Gelehrte aus aller Welt kamen hier zusammen. Im Austausch jüdischer, christlicher und muslimischer Traditionen entstand eine einzigartige Kons-

2 Vgl. Aristoteles: Nikomachische Ethik. 6. Buch, 3-7, 1139b-1141b.

3 Vgl. Martina Roesner: Amor Scientiae und Studium generale. Die Geburt der Idee der Universität im 12./13. Jahrhundert. In: Ludger Honnefelder (Hrsg.): Albertus Magnus und der Ursprung der Universitätsidee. Die Begegnung der Wissenschaftskulturen im 13. Jahrhundert und die Entdeckung des Konzepts der Bildung durch Wissenschaft, Weilerswist 2017, S. 51-76, hier: S. 62f.; Aurelius Augustinus: Die christliche Bildung (De doctrina christiana) [426/427], Stuttgart 2002, Zweites Buch. 
tellation der Wissensvermittlung. Es wird berichtet, »der Engländer Daniel von Morley (1140-1210) [sei], gelangweilt von den einschläfernden Pariser Magistern und ihren sunerträglichen Büchern gen Toledo [aufgebrochen], um die wahre, durch die Araber vermittelte Wissenschaft zu suchen. ${ }^{4}$ Diese Wissenschaft war aber nichts anderes als eine eigenständige Überlieferungslinie der aristotelischen Schriften, verarbeitet beispielsweise von al-Fārābī (ca. 870-950) in seinem Buch Über die Wissenschaften. Dieses Buch wurde in Toledo sowohl von Gerhard von Cremona (1114-1187) als auch von Dominicus Gundissalinus (1110-1190) unter dem Titel De scientiis ins Lateinische übersetzt. Gundissalinus verarbeitete es zudem in seinem eigenständigen Werk De divisione philosophiae (Über die Einteilung der Philosophie), das im 13. Jahrhundert eine entscheidende Rolle in der »disziplinären und v.a. institutionellen Ausdifferenzierung an der Pariser Universität« spielte. ${ }^{5}$ Das entscheidende Moment dieser Traditionslinie liegt nun darin, dass sie die Breite der aristotelischen Wissensgebiete integriert zeigt und unter dem methodischen Primat der Beweislehre ordnet: Bereits al-Fārābī deutete eine Rangfolge der Wissenschaften hinsichtlich dessen an, »in wie strenger Weise die einzelnen Wissenschaften der Beweistheorie des Aristoteles entsprechen. $\ll^{6}$ Dem folgend greift Gundissalinus »die aristotelischen Überlegungen zum Habitus der Apodeixis [Beweis] als der wissenschaftlichen Haltung par excellence auf, die er zugleich differenziert in Hinblick auf die verschiedenen Beweisformen der einzelnen Wissenschaften hin interpretiert. ${ }^{7}$

Diese neue Konstellation konnte für das Verhältnis von christlicher Theologie einerseits und antiken Wissensformen und Philosophie andererseits nicht ohne Wirkung bleiben. Der umfassende Geltungsanspruch dieser

\footnotetext{
4 Alexander Fidora: Aristotelische Wissenschaft als Netzwerk von Wissenschaften. Die Rezeption der aristotelischen Wissenschaftstheorie bei al-Fārābī und Domenicus Gundissalinus. In: Ludger Honnefelder (Hrsg.): Albertus Magnus und der Ursprung der Universitätsidee. Die Begegnung der Wissenschaftskulturen im 13. Jahrhundert und die Entdeckung des Konzepts der Bildung durch Wissenschaft, Weilerswist 2017, S. 77-96, hier: S. 77.

5 Dominicus Gundissalinus: De divisione philosophiae. Über die Einteilung der Philosophie. Hrsg., übers., eingel. und mit Anm. vers. von Alexander Fidora u. Dorothée Werner, Freiburg 2007, S. 41; vgl. S. 49.

6 Franz Schupp: Einleitung. In: Al-Fārābī: Über die Wissenschaften. De scientiis. Nach der lateinischen Übersetzung Cerhards von Cremona, mit einer Einl. u. kommentierenden Anmerkungen hrsg. und übers. v. Franz Schupp, Hamburg 2005, S. XI-LXXXIV, hier S. XLI.

7 Dominicus Gundissalinus: De divisione philosophiae, Freiburg 2007, S. 29.
} 
systematischen Philosophie und ihre Gewissheit und Wahrheit verbürgende Methodologie ließ sich nicht mehr einfach in das platonisch-christliche Bild fügen, in dem die Theologie die gegenständlichen Wissensbereiche der artes als sapientia (Weisheit) überspannte. Eine Reaktion darauf war der Versuch, Teile der aristotelischen Schriften aus den Lehrplänen der Pariser Universität zu verbannen. Eine andere Strategie bestand darin, sich dieses neue Verfahren zunutze zu machen: Insbesondere in Texten aus dem späten 12. und frühen 13. Jahrhundert findet man Versuche, theologische Probleme durch teils ausdrücklich auf Aristoteles rekurrierende - Beweisverfahren zu lösen. Hintergrund sind die Auseinandersetzungen mit anderen Glaubensrichtungen und `Häresien<, die es der Theologie der Zeit geboten erscheinen ließ, neben »Mitteln göttlicher Autorität« auch "gestärkt durch die Waffen notwendiger Beweisgründe « >Irrtümer und >falsche Lehrsätze« zu bekämpfen. ${ }^{8}$

Mit der Rezeption der aristotelischen Beweis- und Wissenschaftslehre eröffnete sich die Möglichkeit, "sich auf einen Boden zu stellen, der nicht mehr primär biblisch-historischer und somit kontingenter Natur, sondern apriorisch und universalgültig ist, [...] mittels lückenloser axiomatischer Darlegung «. ${ }^{9}$ Doch das Schwert der Beweisführung war für die Theologie stets ein zweischneidiges: Wenn ein philosophisch fundiertes Verfahren die theologische Wahrheit zu sichern vermochte, dann war die übergeordnete Stellung der Theologie in ihren Grundfesten zutiefst erschüttert - eine Erschütterung, die letztlich mit einem modernen Wissenschaftsverständnis zugleich die Idee der Universität hervorbrachte - wie es treffend beschrieben wurde: Die Theologie, die die "primär texthermeneutisch orientierte Schriftauslegungstradition seit Augustinus" durch begrifflich-deduktive Vorgehensweisen ersetzt hatte, sah sich in einem zweiten Schritt gezwungen, »in ihrem deduktiven Fortschreiten früher oder später auch die Inhalte der diversen philosophischen Traktate in Betracht zu ziehen, in denen Aristoteles seine umfassende Deutung der Natur, des Menschen und der Wirklichkeit in ihrer Gesamtheit entwirft.« Die Annahme einer einheitlichen Methode führte somit - wir folgen hier den Ausführungen von Martina Rösner - dazu, »dass nun auch Gott als Gegenstand der Theologie prinzipiell mittels derselben Kategorien erkannt werden kann, die für jede andere Form

8 Mechthild Dreyer: More Mathematicorum. Rezeption und Transformation der antiken Cestalten wissenschaftlichen Wissens im 12. Jahrhundert, Münster 1996, S. 149; vgl. insgesamt S. 144-167.

9 Martina Roesner: Amor Scientiae und Studium generale. Weilerswist 2017, S. 66. 
wissenschaftlicher Gegenständlichkeit überhaupt Gültigkeit besitzen.« Und diese neue Konstellation schlägt sich auch institutionell nieder:

»Die Tatsache, dass selbst in der christlichen Theologie keine grundlegend andere facultas cognoscendi zum Einsatz kommt als in den übrigen Wissenschaften, ist somit die ideelle Voraussetzung dafür, dass auch im Rahmen der Universität die im institutionellen Sinne verstandene facultas theologica nicht über, sondern neben den anderen Fakultäten zu stehen kommt und nur noch im Rahmen des ihr eigenen Cegenstandsgebiets, nicht jedoch im Hinblick auf die anderen wissenschaftlichen Disziplinen eine Urteilskompetenz in Bezug auf die Relevanz und Cültigkeit des jeweiligen wissenschaftlichen Diskurses besitzt«. ${ }^{10}$

\section{Episteme: Der Anfang von Wissenschaft?}

Doch halten wir kurz inne - wovon sprechen wir? Es geht uns um Wissenschaft(en) in unserer gegenwärtigen Situation. Um diese im Ansatz zu verstehen, haben wir einen Blick geworfen in die Zeit um 1200 als einer zentralen Umbruchsphase. Es findet sich zwar schon in dieser Zeit ein mittelhochdeutsches Wort wizzen(t)schaft, das ganz allgemein »Wissen, Kenntnis, Kunde « bezeichnete. ${ }^{11}$ Der Diskurs um 1200 wurde jedoch nicht deutschsprachig, sondern lateinisch geführt. Vokabeln wie doctrina (Lehre), artes (Künste) und disciplina spielten hier eine Rolle. Geprägt wurde die Transformation um 1200 aber vom Begriff der scientia ${ }^{12}$, ein Wort, das sich im Englischen und den romanischen Sprachen als science, cientia, ciencia, szientia erhalten hat. Scientia wiederum, das vom Verb scire (wissen; etymologisch von scheiden, unterscheiden) her gebildete Substantiv, diente schon in der Antike zur Übersetzung der griechischen Vokabel episteme. Als nun mit der Übersetzung der Zweiten Analytik die episteme in einer schärferen und

10 Martina Roesner: Amor Scientiae und Studium generale, Weilerswist 2017, S. $75 f$.

11 Siehe Eintrag >Wissen in: Etymologisches Wörterbuch des Deutschen. Q - Z; erarbeitet von einem Autorenkollektiv des Zentralinstituts für Sprachwissenschaft und der Leitung von Wolfgang Pfeifer, Berlin 1989, S. 1984.

12 Vgl. hierzu den Eintrag /Wissenschaft< in: Historisches Wörterbuch der Philosophie, hrsg. v. Joachim Ritter, Bd. 12, S. 902-948, hier insbes. S. $903 \mathrm{ff}$. 
spezifischeren Bedeutung erschien, da prägte sich auch scientia in diesem Sinne aus. Heißt dies jedoch, dass moderne Wissenschaft auf scientia und scientia auf episteme zurückgeht?

In der Wissenschaftsphilosophie und Philosophie der Antike wird dies als selbstverständlich angenommen und fraglos reproduziert. Episteme wird - häufig recht inkonsequent - ins Deutsche mal als Wissen, mal als Wissenschaft übersetzt. Wo es als Wissenschaft übersetzt und verhandelt wird, scheint es zuweilen so, als ob alle Bereiche geistiger Tätigkeit als Wissenschaft gelten, meist aber, dass nur speziell jene Erkenntnis, die zu sicherem Wissen notwendiger Verhältnisse führt, mit diesem Begriff zu bezeichnen sei. Aristoteles hatte sich hier viel klarer ausgedrückt als seine Interpreten: Diese nämlich ergänzen bereits in den Übersetzungen das Wort Wissenschaft an Stellen, an denen es im griechischen Text gar nicht steht und übersetzen zudem ganz unterschiedliche aristotelische Begriffe mit Wissen und Wissen-

\section{BASISWISSEN 1: Episteme im Kontext}

Episteme ist das griechische Wort, das ins Lateinische mit scientia und im deutschen mit den Vokabeln Wissenschaft und Wissen, in die romanischen Sprachen und ins Englische mit science, cientia, ciencia, szientia übersetzt wird. In diesen Übertragungen spiegelt sich eine Traditionslinie der Auffassung von Wissenschaftlichkeit wider, die Wissenschaft mit logischer Beweisführung (apodeixis; demonstratio) identifiziert. Um die Komplexität dieses Überlieferungsgeschehens zu verstehen, ist es allerdings wichtig, sich die Eigenständigkeit und Besonderheit des griechischen Konzepts vor Augen zu führen. Eine Passage aus der Nikomachischen Ethik (einem späten Werk des Aristoteles), macht den Gesamtzusammenhang klar, wie er in die abendländische Tradition eingegangen ist:

Fünf geistige Bereiche seien es, die dem Menschen Wahrheit erschließen: »Kunst [techné], Wissenschaft [episteme], Klugheit [phronesis], Weisheit [sophia] und Verstand [nous] «. Bereits in der Wortstellung zeigt sich in dieser Eröffnung episteme als ein Vermögen unter anderen. Und zwar:

»Was die Wissenschaft [episteme] sei, erhellt, wenn wir die Worte genau nehmen [...], aus folgendem. Wir alle halten dafür, daß das, was man weiß, sich nicht anders verhalten kann, was sich aber anders verhalten kann, von dem weiß man, sobald man es nicht mehr vor Augen hat, nicht, 
schaft. Dies hat den Effekt, dass bereits in die Übersetzungen an vielen Stellen sich moderne Vorstellungen von Wissenschaft eingeschlichen haben, die sich erst hunderte von Jahren später allmählich ausbildeten.

Episteme als ein bestimmter Ausschnitt der Erkenntnisformen des Menschen ist aber noch in anderer Hinsicht etwas ganz anderes als Wissenschaft im modernen Sinne: Sie umfasst nämlich weder die Erfahrung (empeiría), auf der sie gleichwohl basiert, noch das Wissen um die Prinzipien (arché), von denen sie in der Beweisführung ausgeht. Beides findet sich in modernen Vorstellungen von Wissenschaft aber unbedingt integriert. Zu beiden Punkten später mehr. Die Überzeugung, dass es eine einzige Wissensform gibt, die der einzige und alleinige Weg zu wahrer Erkenntnis ist - diese für die Moderne typische Vorstellung - ist also jedenfalls nicht aristotelisch. Unsere heutige Situation ist viel enger an die Entwicklungen um 1200 gebunden als an den aristotelischen Begriff der epistéme.

ob es noch ist oder nicht. Mithin ist, was Gegenstand des Wissens ist, aus Notwendigkeit. Mithin ist es ewig; denn alles, was schlechthin aus Notwendigkeit ist, ist ewig, das Ewige aber ist ungeworden und unvergänglich.« (Aristoteles, Nikomachische Ethik, 6. Buch, 3.2, 1139b, 18ff.)

Und weiter:

»Was sich anders verhalten kann, ist teils Gegenstand des Herstellens [poiesis], teils Gegenstand des Handelns [praxis]«. Klugheit im Handeln und Kunstfertigkeit im Hervorbringen stehen gemäß dieser Konzeption als durch >Vernunft‘ geleitete - wörtlicher wäre: logos-basierte - Haltungen [hexis] auf gleicher Stufe neben der episteme, die ihrerseits einen bestimmten Umgang mit dem logos pflegt.

Für Aristoteles steht also episteme neben anderen Bereichen von Erkenntnis und Vernunft im menschlichen Leben. Wissen, in unserem heutigen Gebrauch des Wortes, findet sich in allen diesen Bereichen, episteme ist dagegen aus der Perspektive unseres heutigen Sprachgebrauchs eine sehr spezielle Form von Wissen, ein Wissen von Gegenständen, unter der Voraussetzung, dass sie nicht anders sein können. Wer Wissenschaft in dieser Traditionslinie betrachtet, impliziert auch ein solches Bild von Erkenntnisgegenständen als notwendige und zeitlose Gegebenheiten (sub specie aeternitatis - in der Gattung des Ewigen (Spinoza)). 


\section{Die Verallgemeinerung der Episteme: Wissenschaft als Beweisen}

Nimmt man ein beliebiges wissenschaftsphilosophisches Studienbuch der Gegenwart zur Hand und schlägt nach, was unter einer wissenschaftlichen Erklärung zu verstehen sei, so trifft man üblicherweise auf eine solche Darstellung: ${ }^{13}$

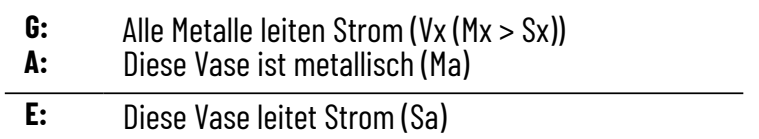

Die so darstellbare Form der wissenschaftlichen Erklärung wird nach Carl Gustav Hempel und Paul Oppenheim, die 1948 einen seitdem tausende Mal zitierten Text zu diesem Thema verfassten, das HO-Schema oder auch das Deduktiv-Nomologische (DN) Modell der Erklärung genannt. Die Gründe, warum dieser Ansatz in der Mitte des 20. Jahrhunderts eine solche Wirkkraft entfalten konnte, liegen in der speziellen Konstellation der Zeit. Sie liegen nicht darin, dass hier etwas bahnbrechend Neues gesagt wäre. Tatsächlich nämlich reproduziert das DN-Modell nichts anderes als das Verfahren der apodeixis, wie es Aristoteles in der Zweiten Analytik ausführlich darstellt. So analysiert etwa Aristoteles das Phänomen, dass Planeten nicht funkeln, so: »es sei etwa C Planeten, B das Nahesein, das A das Nicht-Funkeln; so trifft also das B [Nahesein] auf das C [Planeten] zu und das A [Nicht-Funkeln] auf das B [Nahesein], so dass auch das $C$ auf das A zutrifft [dass also Planeten nicht funkeln] ${ }^{14}$ Das Prinzip, dass Lichter, die nahe sind, nicht funkeln, vermittelt zwischen den beobachteten Planeten und der Aussage, dass sie nicht funkeln, in der Weise, dass die allgemeine Regel die Ursache dafür liefert, warum sie nicht funkeln: weil sie nahe sind. Ganz ähnlich wird die Mondfinsternis auf die Stellung der Erde und auf die Regel, dass Licht durch Gegenstände verringert werden kann, zurückgeführt..$^{15}$

13 Gerhard Schurz: Wissenschaftliche Erklärung. In: Wissenschaftstheorie. Ein Studienbuch, hrsg. v. Andreas Bartels u. Manfred Stöckler, Paderborn 2007, S. 69-88, hier S. 72.

14 Aristoteles: Lehre vom Beweis oder Zweiten Analytik (Organon IV), übers., mit einer Einl. u. Anm. hrsg. v. Wolfgang Detel, Hamburg 2011, Buch I, 13, 78b.

15 Aristoteles: Lehre vom Beweis oder Zweiten Analytik, Buch II, 2, 90 a. 
Es ist angemerkt worden, dass die Wiederentdeckung der Idee der aristotelischen Beweislehre »in der analytischen Philosophie des 20. Jahrhunderts [nur] aufgrund historischer Ignoranz als bedeutende Innovation gefeiert « werden konnte. ${ }^{16}$ Andere vertreten die Ansicht, das DN-Modell sei als eine genauere Ausführung der aristotelischen Überlegungen zu betrachten. ${ }^{17}$ Doch es bleibt dabei: die Grundfigur des Verfahrens, das heute erklären heißt, ist das aristotelische Beweisen, die apodeixis. Bis in die Gegenwart ist der Zusammenschluss von apodeixis-erklären-Wissenschaft in weiten Bereichen der Wissenschaftsphilosophie schlicht vorausgesetzt: »Die Entdeckung der Möglichkeit von Beweis ist zugleich die Entdeckung der Möglichkeit von Wissenschaft «, liest man etwa in einem einschlägigen Lexikonartikel. ${ }^{18}$

Philosophiegeschichtlich lässt sich diese bemerkenswerte Kontinuität gut nachvollziehen. Ausgehend von den Interpretationen der Zweiten Analytik im 13. Jahrhundert wurde das Motiv des Erschließens von Wirklichkeit mittels logischer Ableitung zum Standard dessen, was an Universitäten unter scientia verstanden wurde. Die im 13. Jahrhundert etablierte Vorstellung von Wissenschaften als axiomatisch-deduktive Systeme findet sich wieder bei Petrus Ramus (1543) und René Descartes, der in seinen Regulae ad directionem ingenii (vor 1628) schreibt, dass Wissenschaft aus Prinzipienkenntnis und Deduktion bestehe; Deduktion verstanden als Erkenntnis, die "aus anderem sicher Erkannten mit Notwendigkeit erschlossen wird. ${ }^{19}$ Thomas Hobbes

16 Wolfgang Detel: >Einleitung`zu: Aristoteles: Lehre vom Beweis oder Zweite Analytik, 2011, S. XXXII, Fn. 46.

17 Vgl. Schurz: >Wissenschaftliche Erklärung`2007, S. 69.

18 Vgl. hierzu den Eintrag >Beweis in: Historisches Wörterbuch der Philosophie, hrsg. v. Joachim Ritter, Bd. 1, S. 882-886, hier S. 885.

19 Descartes anerkennt nur zwei Formen von Erkenntnis: Prinzipienerkenntnis durch Intuition und Deduktion; zum Verhältnis der beiden gibt folgende Textstelle Aufschluss: »weshalb wir hier außer der Intuition noch eine andere Art der Erkenntnis angefügt haben, die durch Deduktion geschieht, worunter wir all das verstehen, was aus etwas anderem sicher Erkannten mit Notwendigkeit erschlossen wird. Aber das musste so geschehen, weil das meiste zuverlässig gewußt wird, obgleich es selbst nicht evident ist, wofern es nur aus wahren und erkannten Prinzipien durch eine zusammenhängende und nirgendwo unterbrochene Tätigkeit des Denkens, welches das einzelne deutlich in der Intuition sieht, deduziert ist, nicht anders als wenn wir das letzte Glied einer langen Kette mit dem ersten zusammenhängend erkennen, obgleich unsere Augen nicht mit einem und demselben Blick auf alle Zwischenglieder, von denen jener Zusammenhang herrührt, achten, wenn sie sie nur alle eins nach dem anderen durchmustert haben, und 
bezeichnet (1651) Wissenschaft als Verknüpfung »zu Syllogismen, den Verbindungen einer Behauptung mit einer anderen, bis wir alles kennen«, und stellt klar: »Und eben dies nennt man Wissenschaft. $\aleph^{20}$ Das Konzept einer deduktiv verfahrenden Wissenschaft zieht sich, mit einigen Schwankungen, durch bis in die Philosophie des 20. Jahrhunderts, in dessen logisch-empiristischer Tradition das DN-Modell wissenschaftlichen Erklärens steht.

Doch was hat es mit der Idee von Wissenschaft als Beweistätigkeit auf sich? Im Kanon der Wissenschaftsphilosophie ist die Verknüpfung von Wissenschaft und Beweisen so omnipräsent und selbstverständlich, dass es fast unmöglich scheint, Wissenschaft in irgendeiner Weise anders zu denken. Und doch zeigen sich Bruchlinien in diesem Gefüge, sobald man etwas näher hinsieht. Diese Bruchlinien haben mit dem Verhältnis von Logik und Mathematik ebenso zu tun wie mit den Begriffen Erfahrung, Empirie und Experiment. Auf diese einschneidenden, aber nicht ganz einfach zu sortierenden Unterschiede kommen wir später zu sprechen. Ein Unterschied aber scheint ganz deutlich und unproblematisch zu sein. Wir kennen ihn z.B. schon aus der Einteilung von Schulen und Fächern und es sieht so aus, als würde er ganz natürlich eine Einteilung der Welt der Wissenschaften in zwei Bereiche abbilden: den Bereich der Natur- und den Bereich der Geisteswissenschaften. In den Naturwissenschaften wird erklärt, so heißt es, und in den Geisteswissenschaften verstanden. Erklären und Verstehen: zwei Verfahren, die auf zwei Gattungen von Gegenständen abgestellt zu sein scheinen. Ganz einfach - aber ist das wirklich so natürlich und einfach? Was hat es mit dieser Zweiteilung auf sich? Wie kam es dazu?

wir uns erinnern, daß die einzelnen Clieder mit ihren Nachbargliedern vom ersten bis zum letzten zusammenhängen. «Intuitio bestimmt Descartes offensichtlich im Anschluss an das antik griechische Konzept des geistigen Erfassens, nous (vgl. Kurt von Fritz: Die Rolle des Noein. Um die Begriffswelt derVorsokratiker, Darmstadt 1968. Siehe auch o. BASISWISSEN 1: Episteme im Kontext): »Unter Intuition verstehe ich nicht das schwankende Zeugnis der sinnlichen Wahrnehmung oder das trügerische Urteil der verkehrt verbindenden Einbildungskraft, sondern ein so müheloses und deutlich bestimmtes Begreifen des reinen und aufmerksamen Geistes, daß über das, was wir erkennen, gar kein Zweifel zurück bleibt«. René Descartes: Regulae ad directionem ingenii. Regeln zur Ausrichtung der Erkenntniskraft, krit. revid., übers. u. hrsg. v. Heinrich Springmeyer, Lüder Gäbe, Hans Günter Zekl, Hamburg, 1973, Regel III, Abs. 8 und 5, S. 17-21.

20 Thomas Hobbes: Leviathan, Frankfurt a.M. 1994, S. 36. 


\section{Deutungskämpfe I: Naturwissenschaften versus Geisteswissenschaften - Erklären gegen Verstehen}

Die Standardversion der Geschichte dieser Zweiteilung der Wissenschaften sieht so aus: Die Naturwissenschaften hatten sich mit ihren Forschungen seit Kepler, Galilei und Newton bereits als Wissenschaften etabliert. Als dann im 19. Jahrhundert Sprach-, Geschichts- und Gesellschaftswissenschaften auf den Plan traten und sich ebenfalls als Wissenschaften zu behaupten suchten, lehnten sie das Erklärungsmodell der Naturwissenschaften mit seinen Ableitungen aus allgemeinen Naturgesetzen ab und konturierten eine neue wissenschaftliche Methode, nämlich das Verstehen. ${ }^{21}$

An solchen geläufigen Erzählungen ist viel Wahres, doch meist genügen sie nicht, um begreiflich zu machen, wo der entscheidende Punkt liegt. Wenn das Motiv des Erklärens - soweit wir bisher sehen - im Beweisen, also in der zwingenden Ableitung aus allgemeinen Prinzipien und Gesetzen besteht, worin besteht dann das Motiv des Verstehens? Was macht Verstehen aus? Bei der Charakterisierung kommen schnell wertende Vokabeln ins Spiel: naturwissenschaftliches Erklären gilt als >exakt<, geisteswissenschaftliches Verstehen als >vage‘; im Erklären gehe es rational zu, Verstehen habe dagegen mit Einfühlung zu tun. Solche Wertungen verknüpfen sich mit der Frage, ob Verstehen überhaupt als wissenschaftliches Verfahren gelten kann.

Im Jahr 1862 stellte Johann Gustav Droysen, einer der bedeutenden Historiker des 19. Jahrhunderts, fest:

»wenn es eine Wissenschaft der Ceschichte geben soll, [muss] diese ihre eigene Erkenntnisart, ihren eigenen Erkenntnisbereich haben [...]; und glücklicherweise gibt es zwischen Himmel und Erde Dinge, die [...] nicht erklärt, sondern verstanden werden wollen. $\ll^{22}$

21 Vgl. zu einer Darstellung aus der Perspektive philosophischer Wissenschaftstheorie: Georg H. von Wright: Erklären und Verstehen, Frankfurt a.M. 1993 (3. Aufl.).

22 Johann Gustav Droysen: Historik. Rekonstruktion der ersten vollständigen Fassung der Vorlesungen (1857), Grundriß der Historik in der ersten handschriftlichen (1857/1858) und in der letzten gedruckten Fassung. Textausgabe v. Peter Leyh, Stuttgart/Bad Cannstatt 1977, Beilage: >Erhebung der Ceschichte zum Rang einer Wissenschaft<, S. 451-469, hier S. 461. 
Jede Wissenschaft sei »eine Betrachtungsweise ${ }^{23}$ Die Physik betrachte ihren Gegenstand als »das im Wechsel Gleiche [...]: die Regel, das Gesetz, den Stoff, die Raumerfüllung « ${ }^{24}$; die Geschichtswissenschaft dagegen das »im Gleichen Wechselnde« - zwei Weisen der Betrachtung.

Gut dreißig Jahre später, in seiner Rektoratsrede Geschichte und Naturwissenschaft von 1894, wird Wilhelm Windelband Droysens Überlegungen aufnehmen und mit den Begriffen nomothetisch und idiographisch ebenfalls zwei nicht gegenstandsbedingt, sondern methodisch verschiedene wissenschaftliche Verfahrensweisen unterscheiden. Der Unterschied ist ein Unterschied der Richtung: während in den Naturwissenschaften »das einzelne gegebene Objekt « nur als »Typus, als Spezialfall eines Gattungsbegriffs [...] zur Einsicht in eine gesetzmäßige Allgemeinheit« relevant sei, betrachte die Geschichtswissenschaft »ein Gebilde der Vergangenheit in seiner ganzen individuellen Ausprägung «. ${ }^{25}$ Wie Droysen sieht Windelband den Unterschied der beiden Typen von Wissenschaft nicht als sachlichen, sondern als einen Unterschied in der Sicht- und Herangehensweise:

»Es bleibt möglich und zeigt sich in der Tat, dass dieselben Gegenstände zum Objekt einer nomothetischen und daneben auch einer idiographischen Untersuchung gemacht werden können. Das hängt damit zusammen, dass der Gegensatz des Immergleichen und des Einmaligen in gewissem Betracht relativ ist. Was innerhalb sehr grosser Zeiträume keine unmittelbar merklichen Veränderungen erleidet und deshalb auf seine unveränderlichen Formen hin nomothetisch behandelt werden darf, kann sich darum doch vor einem weiteren Ausblick als etwas nur für einen immerhin begrenzten Zeitraum Cültiges, d.h. als etwas Einmaliges erweisen. ${ }^{26}$

Beispiele, die Windelband anführt, sind Formgesetze von Sprachen, die Physiologie, Formationen der Geologie und die »Entwicklungsgeschichte« der Lebewesen. ${ }^{27}$ Damit wird die Pointe Windelbands klar: Ein wissenschaft-

23 Droysen: Historik. Beilage `Natur und Geschichte〈, S. 470-479, hier S. 477.

24 Droysen: Historik. Beilage `Natur und Geschichte`, S. 470-479, hier S. 474.

25 Wilhelm Windelband: Ceschichte und Naturwissenschaft, Straßburger Rektoratsrede 1894, Straßburg 1904, S. 16.

26 Windelband: Ceschichte und Naturwissenschaft, S. 12.

27 Vgl. Windelband: Geschichte und Naturwissenschaft, S. $12 f$. 
liches Phänomen, wie z.B. die Entstehung des Lebens, kann entweder als historisches Ereignis aufgefasst und beschrieben werden - man versucht dann diesen Prozess, wie er sich tatsächlich zugetragen hat, möglichst detailliert und umfassend zu rekonstruieren -, oder aber als gesetzmäßiger Zusammenhang erklärt werden - was bedeutet, die Bedingungen zu formulieren, unter denen Leben immer und auf die gleiche Weise entstehen würde. Es ist eine Frage des Erkenntnisinteresses, der Blickrichtung, der inneren Ausrichtung und Struktur - damit scheint der Gedanken auf, dass es Unterschiede der Typen von Wissenschaft gibt.

Doch, weit davon entfernt, eine Gleichwertigkeit unterschiedlicher Typen $\mathrm{zu}$ akzeptieren, blieb die Idee der Wissenschaft ein umkämpftes Feld. Zunächst hat sich der englische Sprachgebrauch seit Mitte des 19. Jahrhunderts mehr und mehr auf eine Seite gestellt: Science hießen zumeist nur noch die Naturwissenschaften vom Typ Physik, alle anderen Disziplinen fielen unter $\mathrm{Hu}$ manities. ${ }^{28}$ Und diese Verengung des Begriffs hatte weitreichende Folgen. In England nämlich ging es seitdem nicht um das Verhältnis von zwei Arten von Wissenschaft, es ging nicht um ein Verhältnis auf Augenhöhe, es ging vielmehr um einen Kampf zwischen zwei kulturellen Idealen: dem der naturwissenschaftlich-technischen und dem der klassisch-humanistischen Erziehung.

Nicht zwei Arten von Wissenschaften, sondern zwei Kulturen prallten hier aufeinander - und dies war, als C. P. Snow 1959 den Begriff von den Two Cultures prägte, längst vorbereitet. Bis Mitte des 19. Jahrhunderts nämlich war die klassische Bildung als "proper education of a gentleman ${ }^{29}$ an den englischen Universitäten allein bestimmend. Erst 1850 wagte Cambridge, einen Kurs in natural science anzubieten. 1880 forderte dann Thomas H. Huxley, ein Biologe in den Fußstapfen von Darwin und Hume, das klassizistische Bildungsideal heraus und klagte eine Anerkennung der Naturwissenschaften in ihrem geistigen Wert und in ihrem Beitrag zum nationalen Wohlstand ein. ${ }^{30}$ Wie im Fall Snows einige Jahrzehnte später folgte prompt eine Antwort von Seiten der eta-

28 Zur Geschichte des Gegensatzes von Science und Humanities vgl. Benjamin R. Cohen: Science and humanities: across two cultures and into science studies. In: Endeaver, 1(2001), S. 8-12.

29 Stefan Collini: Introduction. In: C. P. Snow: The two Cultures, Cambridge University Press 1998, S. vii-Ixxii, hier S. xiii

30 vgl. Collini, 1998, S. xiv. 
blierten Bildung: Der »leading man of letters in Victorian England $\aleph^{31}$, Matthew Arnold, konzedierte, dass beide, Wissenschaft und klassische Bildung, ihren Platz haben sollten, dass aber, zur Heranbildung eines »educated man $\aleph^{32}$ das Studium der Literatur, insbesondere der antiken Literatur unverzichtbar sei.

Damit war der Rahmen vorgezeichnet für den Einsatz C. P. Snows, der in seinem scharfen Plädoyer eben nicht zwei Typen der Wissenschaft einander gegenüberstellte, sondern zwei Kulturen, von denen eine das neue Selbstbewusstsein der modernen Naturwissenschaften repräsentierte, die andere die untergehende Welt der gesellschaftlichen Eliten mit ihren traditionellen Bindungen und ihren aristokratischen und großbürgerlichen Bildungsidealen.

Und dieser Unterschied zwischen Science einerseits und gehobener Bildung andererseits liegt auch noch den Debatten um Wissenschaft zugrunde, die in den 1990er Jahren unter dem Titel Science Wars zuerst in den USA geführt, dann auch international zum Thema wurden. Zwar spielen hier auch andere Motive eine Rolle - nämlich die Weise, in der Naturwissenschaften in der Perspektive postmodernen Denkens verstanden wurden (Stichwort: Konstruktivismus - dazu später mehr) - doch ging es noch immer um die Kluft zwischen Science einerseits und anderen intellektuellen Feldern andererseits. Natürlich hatten sich die Machtverhältnisse inzwischen nicht nur in den USA gründlich gewandelt. Als in Deutschland das Bundesministerium für Bildung und Forschung 2007 ein गJahr der Geisteswissenschaften< ausrief, waren dem ein \Jahr der Physik (2000), eines der Lebenswissenschaften (2001), der Geowissenschaften (2002), der Chemie (2003) der Technik (2004), wieder der Physik (2005) und der Informatik (2006) vorangegangen, das Jahr der Mathematik folgte; Geisteswissenschaften als ein einziger großer Topf und unter ferner liefen, wobei Sozialwissenschaften ganz fehlten.

Muss man also sagen, dass die Konkurrenz zwischen Erklären und Verstehen nur eine Episode war? Dass Wissenschaft nun weltweit zu natural science zusammengeschmolzen ist? Ein Blick in die Realität der Universitäten und Forschungseinrichtungen zeigt ein völlig anderes Bild: geistes- und sozialwissenschaftliche Disziplinen und Studiengänge florieren neben naturwissenschaftlichen in großer Breite und Intensität. Ist es also nur eine merkwürdige Prägung des Blicks, eventuell bestimmt durch die angelsäch-

31 Collini, 1998, S. xiv

32 Collini, 1998, S. xiv 
sische Situation und Debatte, die dieses Faktum so leicht vergessen und die Verknüpfung so geläufig macht, dass sie sich fast automatisch einstellt: wer Wissenschaft denkt, denkt - zuerst und zumeist - Naturwissenschaft. Was ist zu tun, um diesen heimlichen Automatismus aufzubrechen? Jedenfalls ist es nötig, sich die Seite des Verstehens gründlicher anzusehen, zu begreifen, wie sie in sich strukturiert ist, wie sie sich im 19. Jahrhundert als alternative Wissenschaftsform konstituierte und wie sie zu dem steht, was sich im 12 . und 13. Jahrhundert als scientia herausschält.

\section{Quellen moderner Wissenschaften II: Die Tradition von Hermeneutik und Interpretation}

Als sich zu Beginn des 13. Jahrhunderts in Paris, Bologna, Oxford und anderen Städten Universitäten zu formieren begannen und sich durch die Rezeption der Zweiten Analytik ein neuer Begriff von Wissenschaft als Beweisführung ausprägte, war das Feld, in dem dies stattfand, natürlich schon bestellt. Denn Erkenntnis, auf die es ankommt, war Erkenntnis im Modus von Textverstehen, von Hermeneutik und Interpretation.

Auch hier vordergründig eine erstaunliche Kontinuität: Sowohl das griechische Verb hermeneuein als auch das lateinische Verb interpretari bezeichnen übersetzen, deuten, vermitteln, auslegen. Während die griechische Vokabel theoretisch marginal blieb, ${ }^{33}$ wurde sowohl in der lateinischen Philologie und Rhetorik als auch insbesondere in der Jurisprudenz das Verb interpretari mitsamt seinen Ableitungen interpres und interpretatio »seit Plautus [254-184] ständiger Besitz der gesamten lateinischen Literatur «. ${ }^{34}$ Des Weiteren wurde die aristotelische Schrift Peri Hermeneias (dt. Lehre vom Satz) ${ }^{35}$ ins Lateinische mit De In-

33 Hans-Georg Gadamer führt in seinem Artikel Hermeneutik (Historisches Wörterbuch der Philosophie, hrsg. v. Joachim Ritter, Bd. 3, S. 1062-1073) nur wenige verstreute Textstellen auf.

34 Manfred Fuhrmann: Interpretatio. Notizen zur Wortgeschichte. In: Sympotica Franz Wieacker, Cöttingen 1970, S. 80-110, hier S. 81.

$35 \mathrm{Ob}$ der Titel von Aristoteles stammt, ist umstritten, auch der Zusammenhang zur Themenstellung der Interpretation ist nur, aber immerhin, indirekt zu rekonstruieren. Vgl. Meinrad Böhl/Wolfgang Reinhard/PeterWalter (Hrsg.): Hermeneutik: Die Ceschichte der abendländischen Textauslegung von der Antike bis zur Cegenwart, Wien u.a. 2013, S. 14-15. 
terpretatione übersetzt, ebenso die Vokabel hermeneia aus der Paulus Textstelle 1. Korinther 12,10 in der Vulgata (um 400 n. Chr.) mit interpretatio. ${ }^{36}$

Gegenstände der Praxis interpretari sind stets Texte oder textförmig gedachte Strukturen, die der besonderen Vermittlung bedürfen. Dies wird in zahllosen Belegstellen deutlich, z.B. wenn Cicero erklärt, dass für das wahre, naturrechtliche Gesetz »nicht nach einem Erklärer oder Ausleger [explanator aut interpres] « gesucht werden müsse ${ }^{37}$ oder, etwa 600 Jahre später, wenn in den von Iustitian in Auftrag gegebenen Institutionen (entworfen als Einführung und Lehrbuch des römischen Rechts) zu lesen ist: »Von Alters her war es nämlich so eingerichtet, dass es Männer gab, die das Recht öffentlich auslegen sollten [publice interpretarentur] «. ${ }^{38}$ Diese Longue-Durée-Kontinuität, die sich beliebig über Bacon in die Neuzeit mit Belegstellen verlängern ließe, ${ }^{39}$ zeigt, dass eine bestimmte Konzeptualisierung von Erkenntnis nicht erst in der modernen Bibelauslegung und Philologie, sondern schon mit der Rezeption insbesondere der lateinischen Juristik und Rhetorik sich als eine eigenständige Könnerschaft, eine ars etabliert hatte.

\section{Verstehen als Arbeit an sich selbst und den eigenen Konzepten}

Für die Tradition der christlichen Hermeneutik spielte De Doctrina Christiana, ein Werk, das Augustinus um 400 n. Chr. verfasste, eine entscheidende Rolle. ${ }^{40}$ Der Titelbegriff doctrina ist nicht leicht zu übersetzen, da es in dem Werk weniger um eine Lehre im Sinne einer Doktrin, denn um eine methodische Reflexion geht. ${ }^{41}$ Diese Methode setzt Augustinus explizit ins Verhältnis zu scientia, die er als eine bestimmte Stufe in den Erkenntnisweg der doctrina

36 Vgl. Gerhard Kurz: Hermeneutische Künste. Die Praxis der Interpretation, Stuttgart 2018, S. 7.

37 Marcus Tullius Cicero: De re publica. Vom Staat. Übers.u. hrsg. v. Michael von Albrecht, Stuttgart 2013, 3. Buch, Kap. 22; S. 201.

38 Corpus luris Civilis. Die Institutionen, hrsg. v. Rolf Knütel, Berthold Kupisch, Sebastian Lohsse u. Thomas Rüfner, Heidelberg u.a. 2013 (4. Aufl.), Erstes Buch, Zweiter Titel, 8, S. 4.

39 Vgl. hierzu: Kurz: Hermeneutische Künste 2018; Böhl u.a.: Hermeneutik 2013.

40 Zur Rolle von Augustinus in der Hermeneutik vgl. Böhl u.a.: Hermeneutik 2013, S. 162-164.

41 Karla Pollmann: Nachwort. In: Aurelius Augustinus: Die christliche Bildung (De doctrina christiana). Übersetzung, Anmerkung und Nachwort v. Karla Pollmann, Stuttgart 2013, S. $260 f$. 


\section{BASISWISSEN 2: Hermeneutik - verstehende Wissenschaft}

Der Begriff Hermeneutik wurde im 17. Jahrhundert eingeführt zur Bezeichnung eine Methoden- oder Kunstlehre des Verstehens (Erster Titel: Hermeneutica Sacra sive Methodus exponendarum S. Literarum, Dannhauer 1654). Mit >Kunst $<$ ist hier im Anschluss an das griechische techné und das lateinische ars eine Könnerschaft gemeint, die eine innere Systematik aufweist, somit lehr- und lernbar ist. Im Anschluss an diesen Impuls entwickelte sich eine umfassende Tradition der Verstehenslehren, die mit dem Begriff der Hermeneutik assoziiert war. Sie bündelte die Traditionsstränge der antiken und mittelalterlichen Interpretationslehren wie sie in der Philosophie, Philologie, Juristik und Rhetorik bereits vorhanden waren.

Begrifflich und systematisch nahm sie dabei ein Grundmotiv aus der aristotelischen Schrift Peri Hermeneias in Gebrauch, nämlich das Problem der Übermittlung und des Nachvollzugs eines ursprünglichen Satzsinns. Als eine Bewegung der Systematisierung und Verwissenschaftlichung prägte sie schon früh die hermeneutischen Grundmotive: die Interpretation aus der Beziehung von Teil und Ganzem, die sich anpassende, nachahmenden Rekonstruktion und die kritische Reflexion, auch des eigenen Standpunkts.

Im 19. Jahrhundert wurde die philologische Hermeneutik (Schleiermacher) und die juristische Hermeneutik (Savigny) systematisch ausgebaut, daneben entstand eine hermeneutische, interpretative Geschichtswissenschaft (Droysen); im 20. Jahrhundert eine ,Verstehende Soziologie`(Weber) und >Hermeneutische Ethnologie، (Geertz). Die >Philosophische Hermeneutik^ (Heidegger, Gadamer, Ricoeur, Taylor) entfaltet eine Interpretation des menschlichen Weltverhältnisses als eines, das von Grund auf durch Verstehen konstituiert wird. In ihrer Weiterentwicklung wurden auch das Nicht-Verstehen und die Grenzen des Verstehens zu zentralen Themen.

integriert. Unter scientia fallen sämtliche Wissensdisziplinen der Antike wie Geschichtsschreibung, Astronomie, Dialektik und Mathematik. Doch keine 
dieser Disziplinen ist für Augustinus Selbstzweck, sie stellen lediglich Kontextwissen zum Verständnis der Heiligen Schrift bereit.

Das antike Motiv der Deutungsbedürftigkeit von Texten wird in der christlichen Tradition so transformiert, dass deren Interpretationsbedürftigkeit auf eine Steigerung des Erkenntnisbemühens hin angelegt ist. Augustinus beantwortet die Frage danach, warum Gottes Rede mit dunklen Stellen und Allegorien versetzt sei, so:

»Die nämlich nicht geradewegs finden, was sie suchen, mühen sich unter Hunger ab. [...] Denn prächtig und heilsam hat der HI. Geist die HI. Schrift so umgeformt, dass er mit klareren Stellen dem Hunger begegnet, mit den dunkleren aber den Überdruß vertreibt. Fast nichts nämlich wird in jenen dunklen Stellen aufgestöbert, was nicht anderswo in klarster Weise ausgedrückt gefunden werden kann.« ${ }^{42}$

Gut tausend Jahre später, inzwischen hatte sich eine protestantische Verstehenslehre ausgebildet, handelt Matthias Flacius in ganz ähnlicher Weise von den »Ursachen der Schwierigkeit der Heiligen Schrift« und kommt zu dem Schluss:

»Vieles ist selbst den Frommen verborgen, damit sie um so eifriger die Heilige Schrift erforschen und eine klarere Offenbarung erstreben. [...] Schließlich sollen wir hier Gott und seine Mysterien wie in einem Rätsel und unvollkommen erkennen, im anderen Leben aber vollständig und von Angesicht zu Angesicht sehen. $^{43}$

»Wie in einem Rätsel«: Diese Formulierung bringt ein zentrales Motiv in der Tradition der Verstehenslehren zum Ausdruck. Denn wenn ein Erkenntnisgegenstand in rätselhafter Weise gegeben ist, dann erfordert dies nicht nur intensive, sich hineinversetzende Beschäftigung mit ihm, es erfordert auch eine bestimmte Forschungsweise: Während das Erklären im Sinne des Beweis-

42 Augustinus: Die christliche Bildung (Dedoctrina christiana) [426/427], Stuttgart 2002, Zweites Buch, VI.7.14, S. 51.

43 Matthias Flacius Illyricus: De ratione cognoscendi sacras literas. Über den Erkenntnisgrund der Heiligen Schrift, übers., eingel. u. m. Anm. vers. v. Lutz Geldsetzer, [es handelt sich bei dieser Textausgabe um den Anfang des zweiten Teils der Clavis scripturae sacris von 1567, vgl. das Vorwort des Herausgebers], Düsseldorf 1968, S. 23. 
schlusses so etwas wie eine geistige Maschine entwirft, in der sich das eine aus dem anderen zwangsläufig ergibt, bewegt sich das Rätsellösen im Gestus des Suchens. Stets muss nach Möglichkeiten gesucht werden, es muss arrangiert und probiert werden, bis sich eine Lösung zeigt. Friedrich Schleiermacher drückt die Zugangsweise des Verstehens in der Formulierung aus, dass wir

»öfter vom Ende zum Anfang zurückkehren und das Auffassen ergänzend von neuem beginnen; je schwieriger die Cliederung des Ganzen zu fassen ist, desto mehr suchend ihr vom Einzelnen aus auf die Spur [...] kommen, je reichhaltiger und bedeutsamer das Einzelne ist, um so mehr suchend es vermittelst des Ganzen in all seinen Beziehungen [auffassen]. ${ }^{44}$

Suchen heißt im Griechischen zetein. Um dieses Moment des Suchens im Verstehen herauszustellen, spricht man auch von einer "zetistischen «, einer "forschenden " Interpretation. ${ }^{45}$ Dieses suchende Moment sei nämlich gemeint, wenn win der hermeneutischen Literatur von der Erfahrung, dem Wissen, der Kongenialität, dem Takt und >Fingerspitzengefühl, von Intuition und Divinationsgabe des Hermeneuten gesprochen wird «. ${ }^{46}$ Das Konzept des Forschens stammt nicht aus den Beweislehren, in denen es ums Demonstrieren geht, es stammt aus den Verstehenslehren: denn die Worte research und forschen bezeichnen eine intellektuelle Haltung des Suchens und des Fragens, die dann auftaucht, wenn man wissen will, wie Dinge miteinander zusammenhängen, wie sich aus Teilen ein Gesamtzusammenhang ergibt oder woraus ein Zusammenhang besteht.

Vor diesem Hintergrund ist offensichtlich, welches Spannungsfeld sich eröffnete, als die Zweite Analytik zu versprechen schien, über die formale Korrektheit logischer Schlüsse hinaus einen Weg zu bieten, auch inhaltlich aus wahren Prinzipien auf wahre Folgerungen übergehen zu können. Für Augustinus bestand scientia (bestenfalls) in hilfreichen Kenntnissen, nun konnte es so scheinen, als ob in scientia selbst der Weg zur Wahrheit liege.

44 Friedrich Schleiermacher: Über den Begriff der Hermeneutik mit Bezug auf F.A. Wolfs Andeutungen und Asts Lehrbuch. In: Hermeneutik und Kritik, hrsg. u. eingel. v. Manfred Frank, S. 309-346, hier S. 333f.

45 Vgl. Lutz Geldsetzer: >Einleitung‘zu Matthias Flacius Illyricus: De ratione cognoscendi sacras literas. Über den Erkenntnisgrund der Heiligen Schrift, Düsseldorf 1968, S. XIVff.

46 Geldsetzer: >Einleitung〈1968, S. XV. 
Genauer: als ob es eine intellektuelle Technik gebe, die, richtig angewandt, in gleichsam mechanisch-zwingender Weise Wahrheit freilegt.

Für das Verhältnis von Erklären und Verstehen ist der Kontrast zwischen einer scientia, die als Technik Wahrheiten demonstriert und einer sapientia, die als Einsicht aus einem Weg der Selbstveränderung und Selbstläuterung resultiert, von grundlegender Bedeutung. Denn es ist dieses Moment der Selbstveränderung des Erkennenden in der Erkenntnis, das als ein Grundmotiv der Tradition der Verstehenslehren in Rechnung zu stellen ist. Erkennen bedeutet hier, sich auf einen Weg zu machen, auf dem das System der eigenen Überzeugungen grundlegend verwandelt wird.

\section{Quellen moderner Wissenschaften III: Die Tradition der Naturalis historia - Beschreiben, Entdecken, Klassifizieren}

Mit dem Erklärungs- und dem Verstehensparadigma ist es nicht getan: Es gibt noch einen dritten Strang, in dem sich Ideen zu Wissenschaft gebündelt und tradiert haben. War das erste Modell charakterisiert durch die Verben beweisen, demonstrieren und erklären, das zweite durch verstehen, rätsellösen, rekonstruieren, so treffen wir nun auf eine dritte Familie von Vokabeln: beobachten, beschreiben, sammeln, zuordnen, klassifizieren. Wer beobachten und sammeln will, muss finden und unterwegs sein. Deshalb gehört zu diesem Thema der Begriff des Entdeckens und damit auch der Entdeckungsreise, der Forschungs- und Erkundungsfahrt, der Expedition. Dass Wissenschaft in erster Linie mit Entdeckungen und dem Sammeln und Ordnen von Fakten $\mathrm{zu}$ tun hat, diese Idee ist sicherlich nicht weniger tief verankert als diejenige, dass es bei Wissenschaft ums Beweisen oder Verstehen geht. Deshalb ist auch zu erwarten, dass sie ebenfalls über eine lange und ehrwürdige Tradition verfügt. Und tatsächlich finden wir hier einen kanonischen Text, der für die gesamte Traditionslinie namensgebend wurde: die Naturalis Histori$a e$, verfasst von Plinius Secundus (der Ältere), einem römischen Gelehrten in der Zeit bis 77 n. Chr. In 37 Bänden versammelt das Werk weite Bereiche des Wissens der Antike aus den Sachgebieten Kosmologie, Astronomie, Meteorologie, Geologie, Geographie, Anthropologie, Zoologie, Botanik, Pharmakologie, Mineralogie, Metallurgie, Plastik und Malerei. Grundlage der Abhandlungen sind tausende von Exzerpten von über 400 Autoren, die auch 


\section{BASISWISSEN 3: Historia - ein missverständliches Wort}

Mit unserem Wort Historie verbinden wir Gedanken an Geschichte im Sinne eines zeitlichen Ablaufs. Das griechische Wort historia bedeutet aber ganz allgemein das Erforschen und das durch Befragen und Beobachten Erkannte und damit auch die Kenntnis, die Kunde und auch der Bericht von etwas. Die Libros Naturalis Historiae des Gaius Plinius Secundus sind also als Naturgeschichte im Sinne einer $\mathrm{Zu}$ sammenstellung von Erkenntnissen der Naturforschung, im Sinne einer Naturkunde und ihrer Darstellung zu verstehen.

Anknüpfend an Plinius entfaltete sich seit dem 18. Jahrhundert ein Wissenschaftstypus, der in der Sammlung und Systematisierung von Naturphänomenen bestand, besonders der Botanik, Zoologie, Mineralogie, Medizin und Anthropologie. Die Begriffe Naturgeschichte, Histoire Naturelle und Natural History begegnen heute vielfach im Zusammenhang mit naturwissenschaftlichen Sammlungen und naturkundlichen Museen.

Eine verzeitlichende Verwendung des Begriffs findet sich prominent erst im 18. Jahrhundert, etwa in Kants Allgemeine Naturgeschichte und Theorie des Himmels (1755) - und zwar in der Programmatik, die >Ordnung der Natur durch >Auswicklung`, ausgehend von einem chaotischen Anfangszustand zu begreifen, somit: »sich ein wohlgeordnetes Ganze erzeugen zu sehen« (S. 232; A XXII; XXIII).

In der Folge konnte sich Naturgeschichte mit dem Konzept einer Evolution (>Auswicklung`) verbinden: Naturgeschichte ließ sich nun als Entwicklungsgeschichte des Weltalls, der Lebewesen, des Menschen und menschlicher Eigenschaften verstehen.

namentlich genannt werden. Auch eigene Kenntnisse, teils erworben auf weitläufigen Reisen, flossen ein - und Plinius bedauerte, dass die griechische Kultur der Forschungsreisen in Rom aus der Mode gekommen war. ${ }^{47}$

47 Vgl. Arno Borst: Das Buch der Naturgeschichte: Plinius und seine Leserim Zeitalter des Pergaments (Abhandlungen der Heidelberger Akademie der Wissenschaften/Philosophisch-historische Klasse), Heidelberg 1994, S. 19 u. 24. 
Das umfangreiche Werk wurde durch zahlreiche Abschriften das gesamte Mittelalter durch tradiert und blieb auch in der Neuzeit ein wichtiger Bezugspunkt naturwissenschaftlichen Wissens ${ }^{48}$ Allein zwischen 1469 und 1799 sollen 2222 komplette und 281 Auswahlausgaben $\star^{49}$ erschienen sein. Die Verbreitung des Werks begründete eine Tradition, die ihren Höhepunkt im 18. und frühen 19. Jahrhundert erreichte.

Am Anfang einer Kette großer und bis heute für die Wissenschaften wichtiger Namen steht Carl von Linné, der schwedische Gelehrte, der ab 1737 in den zwölf - jeweils ausgeweiteten - Auflagen seines Werk Systema Naturae schließlich etwa 7700 Pflanzen-, 6200 Tier- und 500 Mineralienarten beschrieb und klassifizierte. Parallel verfasste der französische Naturforscher Georges Louis Leclerc Buffon zwischen 1749 und 178836 Bände der (später noch ergänzten) Histoire naturelle générale et particulière. 1755 erschien Immanuel Kants Allgemeine Naturgeschichte und Theorie des Himmels und 1790 machte sich Alexander von Humboldt zunächst mit dem Naturforscher und Weltreisenden Georg Forster auf zu einer Forschungsreise an den Niederrhein, dann zu einer großangelegten Amerikareise mit verschiedenen Expeditionen zwischen 1799-1804, die schließlich in das 1845-1862 publizierte Werk Kosmos - Entwurfeiner physischen Weltbeschreibung mündete.

Diese Epoche des Reisens und Beschreibens war zugleich eine der großen Sammlungen. Gesteine, Pflanzen, Insekten, Muscheln, Geräte, anatomische Präparate: nicht nur der Adel repräsentierte sich durch große Kunst- und Naturalienkabinette, sondern auch das Bürgertum entwickelte eine Leidenschaft, Sammlungen aller Art anzulegen. ${ }^{50}$ Die Rolle, die die Sammlungen für die Wissenschaft spielten, lässt sich wiederum an Linné belegen: Sein flexibler Herbarschrank, der so angelegt war, dass seine Fächer nicht fix waren, sondern nach Bedarf eingeteilt und ergänzt werden konnten, kann als ein Werkzeug der Systematisierung betrachtet werden. So zeigt sich in diesem Möbelstück »eine neue empirische Wissenschaft, die sich auf Beob-

48 Vgl. Gerhard Winkler: Anhang; Naturalis Historia, in: Plinius Secundus, Gaius: Naturkunde (lateinisch - deutsch), hrsg. u. übers. v. Roderich König in Zus. m. Joachim Hopp u. Wolfgang Glöckner, Düsseldorf/Zürich 1997 (2. Aufl.), S. 353-385, hier S. 373.

49 Winkler: Anhang; Naturalis Historia, S. 373.

50 Vgl. Henning Bock: BürgerlicheSammlungen im18. Jahrhundert. Bildungdurch Anschauung. In: Annemarie Gethmann-Siefert/Elisabeth Weisser-Lohmann/Bernadette CollenbergPlotnikov (Hrsg.): Kunst als Kulturgut. 2, »Kunst«und»Staat«., München 2011, S. 33-50. 
achten und Beschreiben, Sammeln und Klassifizieren stützte und die damit im 18. Jahrhundert die Grundlage der modernen Systematik auf Grund von Fakten und Objekten legte. $\aleph^{51}$

Naturgeschichte, Histoire naturelle, Natural History: solche Titel lassen an längst vergangene Wissenswelten denken. Aber tatsächlich ist das Erheben von Daten und ihre Integration in Ordnungssystemen (Taxonomien) nach wie vor ein Kernbereich wissenschaftlicher Tätigkeit. Wenn es in der biologischen Systematik z.B. heute darum geht, mittels DNA-Analysen Verwandtschaftsbeziehungen zwischen Pflanzen- und Tierarten nachzuzeichnen, dann geschieht hier, was die Aufgabenstellung anbelangt, etwas sehr Ähnliches wie in den Hochzeiten der Naturgeschichte. Einblick und Überblick über die Ordnung der Dinge - so der deutschsprachige Titel eines Werks von Michel Foucault, in dem er das taxonomische Denken des 18. Jahrhunderts analysierte ${ }^{52}-$ zu gewinnen, ist nach wie vor das Bestreben dieses Typs wissenschaftlicher Arbeit. Schon die Naturalis Historiae ist keine bloße Zusammenstellung von Wissen, sondern folgt einer inneren Systematik der Gegenstände. ${ }^{53} \mathrm{Zu}$ den frühen Klassifikationssystemen der belebten und unbelebten Natur sind inzwischen eine Vielzahl neuer Systematiken hinzugekommen: Sprachen, chemische Elemente, Krankheiten, literarische Texte, Ethnien, Artefakte - für alle diese Sachen gibt es Einteilungen und tabellarische Gliederungen, die Ordnungssysteme darstellen.

Als mediale Form erlebte die Anordnung von "Information in tabellarischer Disposition $\aleph^{54}$ im 18. Jahrhundert ihre Blüte. Mit dem Anwachsen von Daten - 1623 habe man, so wird berichtet, 6000 botanische Arten, 1694 dann schon 10146 und nur zehn Jahre später 18655 gezählt ${ }^{55}$ - kommt allerdings auch die tabellarische Darstellung in vielen Bereichen an ihre Grenzen. Das gab Anlass, von einem »Ende der Naturgeschichte« im 19. Jahrhundert zu sprechen. ${ }^{56}$

51 Bock 2011, hier S. 33.

52 Michel Foucault: Die Ordnung der Dinge. Eine Archäologie der Humanwissenschaften [Les mots et les choses], Frankfurt a.M. 1971 [1966].

53 Vgl. Eugenia Lao: Taxonomic Organization in Pliny's Natural History. In: Papers of the Langford Latin Seminar, 16. (2016), S. 209-246.

54 ArndtBrendecke:Information intabellarischer Disposition. In: FrankGrunert/AnetteSyndikus (Hrsg.): Wissensspeicher der Frühen Neuzeit: Formen und Funktionen, Berlin 2015, S. 43-59.

55 Wolf Lepenies: Das Ende der Naturgeschichte: Wandel kultureller Selbstverständlichkeiten in den Wissenschaften des 18. und 19. Jahrhunderts, München 1976, S. 55.

56 Wolf Lepenies: Das Ende der Naturgeschichte München 1976. 
Doch was hier zu Ende ging, war lediglich die Idee, dass sich die Ordnung der Natur, zusammengefasst in einem umfassenden Werk, überblickshaft als Ganzes darstellen lässt. Was dagegen seit dem 17. Jahrhundert immer deutlicher Kontur gewann, war die Methode, möglichst umfangreiche Daten zu erheben und aus der Organisation dieser Daten Erkenntnisse zu gewinnen. Natürlich kam hier seit dem 19. Jahrhundert auch die Statistik ins Spiel. Zunächst war Statistik nur die Aufzeichnung von verwaltungstechnisch relevanten Daten wie Bevölkerungszahl, Geburten und Sterbefälle gewesen. Doch mit dem Einsatz mathematischer Verfahren entwickelte sich daraus ein methodisches Arsenal, das tief in unterschiedliche Wissenschaften hineinwirkte und auch darauf zielte, in Datenmaterial Regelmäßigkeiten aufzufinden. Auf das Verhältnis von Statistik und Wissenschaft kommen wir später noch ausführlicher zu sprechen.

Fassen wir zusammen: In der Traditionslinie der Naturgeschichte zeichnet sich ein Bild von Wissenschaft ab, das von den Konzeptionen der Scientia und der Verstehenslehren deutlich unterschieden ist. Wissenschaft als Erhebung und Darstellung von Faktenwissen ist offensichtlich eine ganz andere Vorstellung als die eines Beweisens, das durch Ableitung aus Prinzipien zustande kommt, oder die eines Verstehens, das sich in einen Prozess des suchenden Verknüpfens und Rätsellösens involviert.

Alle drei Bilder von Wissenschaft haben tiefe Wurzeln in der abendländischen Geschichte des Denkens und ebenso offensichtlich tragen alle drei nach wie vor Früchte. Müssen wir also von drei Gattungen von Wissenschaft sprechen? Die durch unüberschreitbare Schranken voneinander getrennt sind? Doch wie sollte sich diese Dreiteilung äußern? Offensichtlich lässt sich ja das Spektrum der gegenwärtigen wissenschaftlicher Disziplinen kaum in dieses Raster fügen. Vielleicht müssen wir noch einen Schritt weiter gehen, und unter den Oberflächen dieser Traditionen den Motiven und Methoden nachspüren, die die wissenschaftliche Praxis noch konkreter prägen. Was hat es mit der Mathematisierung als Kennzeichen von Wissenschaft auf sich? Was mit dem Experiment? Was bedeutet Analyse und Synthese? Was Induktion und Deduktion? Wohin gehört das Rekonstruieren und wohin das Modellieren? Welche Rolle spielt das Interpretieren? Welche die Kritik? Es ist Zeit, hier Klarheit und Orientierung zu finden - und dazu bietet das Gerüst der drei Wissenschaftstraditionen eine gute Voraussetzung. 


\section{Auffächerungen in den drei Wissenschaftstraditionen}

\section{Noch einmal zum Beweisen: Die Rolle der Mathematisierung}

»Alles was Gegenstand des wissenschaftlichen Denkens überhaupt sein kann, verfällt, sobald es zur Bildung einer Theorie reif ist, der axiomatischen Methode und damit unmittelbar der Mathematik. ${ }^{1}$

- das lesen wir bei David Hilbert, einem der führenden Vertreter der modernen Mathematik zu Beginn des 20. Jahrhunderts. Dass Wissenschaft idealerweise exakte Wissenschaft sein soll, und Exaktheit sich in mathematischen Verfahren ausdrückt, ist aber auch hundert Jahre später verbreitete Meinung. Doch was hat es mit dieser Meinung auf sich? Worin begründet sie sich? Und was ist das Kernmotiv dieser Auffassung?

Auch hier wird uns eine historische Perspektive, ein Blick auf die Genese dieser Denkfigur helfen. Die axiomatische Methode, von der Hilbert spricht, hat eine ihrer wichtigsten Quellen - wie könnte es anders sein - in der Zweiten Analytik des Aristoteles. Allerdings geht es dort, wie wir gesehen haben, nicht um eine im engeren Sinne mathematische, sondern um eine allgemeine Beweislehre. Beispiele aus der Geometrie und aus der Arithmetik stehen hier neben Beispielen aus der Astronomie und der Politik. Im Kontext seiner allgemeinen Beweislehre gibt Aristoteles dem Begriff axioma in der Zweiten Analytik eine spezifische Bedeutung, nämlich den eines Ausgangspunktes und einer Voraussetzung von erschließbarem Wissen. ${ }^{2}$

1 David Hilbert: Axiomatisches Denken (1918). In: David Hilbert: Hilbertiana. FünfAufsätze von David Hilbert, Darmstadt 1964, S. 1-11, hier S. 11.

2 Wie grundsätzlich diese Voraussetzungen zu verstehen sind, darüber streitet die Aristoteles-Forschung. Sind damit die jeweiligen Prinzipien gemeint, wie, in den Beispielen oben, 


\section{BASISWISSEN 4: Axiome - Grundlagen von Schlussfolgerungen}

Bei Aristoteles hieß axioma zunächst schlicht: der im Disput eingesetzte Satz, für den Zustimmung eingefordert wird - im Sinne von >Wir können doch davon ausgehen, dass .... (vgl. Topik 155b) In den Analytiken wird der Begriff nun zugespitzt auf dasjenige, woraus bewiesen wird. (vgl. 76b 14) Es geht dabei um die allgemeinen und prinzipiellen Voraussetzungen, die einem beweiskräftigen Syllogismus zugrunde liegen. In der Zweiten Analytik wird der Begriff Axiom so eingeführt: Ein Axiom sei ein im logischen Schließen (Syllogismos) eingesetztes Prinzip, das zum Wissenserwerb notwendig ist. (vgl. 72a 15ff.) Dabei werde das Wissen im Modus des Beweisens durch dreierlei bestimmt:

1. das Gesetzte (die vorausgesetzten Gegenstände, um deren Eigenschaften es geht);

2. die Axiome, aus denen bewiesen wird, und

3. die Eigenschaften, die das Bezeichnete annimmt. (Vgl. 76bi3ff.)

Hören wir uns - im Originalton Aristoteles - an, wie sich die drei Dinge in der Erkenntnis durch ein logisches Beweisverfahren wiederfinden. Frage ist (es geht um den Perserkrieg) (vgl.94a 36f.)

»Was war die Ursache, dass die Athener mit Krieg überzogen wurden? Weil sie mit denen aus Eritrea die Stadt Sardis angriffen. Denn das gab

dass Lichter nur in der Ferne funkeln oder dass beleuchtete Gegenstände Schatten werfen, oder nur Voraussetzungen allgemeinster Art wie der Satz vom Widerspruch, oder, dass ein Abzug des Cleichen vom Gleichen wiederum Gleiches ergibt? Jedenfalls geht es um Wahrheiten, die - gleichsam - für sich selbst sprechen und als Crundlage einer logischen Schlussfolgerung (Syllogismos) dienen - sei es im Disput, sei es in einem Beweisverfahren. Umstritten ist auch, ob der Begriff des Axioms aus der Mathematik in die Philosophie oder aus der Philosophie in die Mathematik gelangte. Die Geschichte der wechselseitigen Übernahmen ist schon in der Antike ziemlich verwickelt. Vgl. Árpád Szabo: Anfänge der griechischen Mathematik, Wien 1969, S. 302-416; Aristoteles-Handbuch. Leben-Werk-Wirkung, hrsg. v. Christof Rapp u. Klaus Corcilius, Stuttgart 2011, insbes. S. 47-52. 
den ersten Anstoß. Krieg soll A sein, zuerst angreifen B, Athener das C. Es kommt also B dem C zu, zuerst angreifen den Athenern, $A$ aber dem B; denn man führt gegen diejenigen Krieg, die zuerst eine Unbilde zugefügt haben. Mithin kommt dem B das A zu, daß man gegen die, die zuerst angefangen haben, Krieg führt, dieses $B$ aber den Athenern, weil sie zuerst angefangen haben.«(94a,b übers. Rolfes)

Legt man diesen Syllogimos in die drei genannten Elemente aus, dann stellt sich das folgendermaßen dar:

1. Die Gegenstände sind: Krieg A; Zuerst angreifen B, Athener C.

2. Das Axiom (axioma) ist: Wer zuerst angreift und Unrecht tut, wird mit Krieg überzogen

3. Zukommende Eigenschaften: den Athenern kommt die Eigenschaft $\mathrm{zu}$, mit dem Krieg begonnen zu haben.

So bilden die drei genannten Elemente gleichsam die Spielaufstellung. Nun muss nur noch gespielt werden. Dabei ist (3.) schon der erste Zug, aus dem sich das Folgende notwendig ergibt: Denn setzt man die Athener als Kriegsbeginner ein, so ergibt sich unter dem allgemeinen Prinzip, dass, wer Wind sät, Sturm erntet (um eine sprichwörtlich gewordene Version des Grundsatzes einzusetzen), dass die Athener Kriegsgewalt erleiden - und zwar notwendig.

Die bereits in der Antike nicht ganz einfach zu entwirrenden Verwicklungen um den Begriff Axiom traten in eine neue Phase, als im 12. Jahrhundert nicht nur die Zweite Analytik wieder auftauchte, sondern zeitgleich auch die Elemente des Euklids, das einflussreichste Lehrbuch der antiken Geometrie, vollständig übersetzt und zugänglich wurde. ${ }^{3}$

3 Vgl. Mechthild Dreyer: More Mathematicorum. Rezeption und Transformation der antiken Gestalten wissenschaftlichen Wissens im 12. Jahrhundert, Münster 1996, S. 82-91. 
Robert Grosseteste (1168-1253), der in Oxford einen der frühesten Kommentare zur Zweiten Analytik verfasste, gilt - gemäß einer einflussreichen Studie aus den 1950er Jahren- als einer der Begründer der modernen, experimentellen Wissenschaften. ${ }^{4}$ Diese enthusiastische Einschätzung ist zwar inzwischen einem nüchternen Blick gewichen, ${ }^{5}$ aber an einem Punkt bricht Grosseteste tatsächlich mit dem aristotelischen Denken - und dies ist der Punkt der Rolle der Mathematik. Waren für Aristoteles die Beispiele aus der Geometrie Beispiele unter anderen für das übergreifende Verfahren der apodeixis, so wird bei Grosseteste das geometrische Demonstrieren zur Idealform der Wissensgenese. Im Rahmen der umfassenden aristotelischen Wissenschaftslehre, wie sie im 12. und 13. Jahrhundert aufgefasst wurde, rückt nun die Geometrie an die Stelle einer sprachlichen Syllogistik. ${ }^{6}$ Nach der Weise der Geometrie - »more geometrico « zu verfahren, wird jetzt zu einem Slogan. Hinter diesem zunächst kaum merklichen Paradigmenwechsel steht eine Zusammenführung mit der Euklidischen Geometrie: »Grosseteste is not simply giving a reading of Aristotle's Posterior Analytics alone. Indeed, it is a reading of Aristotle-Euclid together «. ${ }^{7}$

Doch was hat es mit Euklid und dem geometrischen Verfahren auf sich?

Euklid, wohl etwas jünger als Aristoteles, hatte das mathematische Wissen seiner Zeit in einer systematischen Darstellung integriert. Zwar taucht in diesem Text das Wort Axiom nicht auf, doch hatten sich die historischen Verwicklungen in systematische Parallelen niedergeschlagen, die dann in der

4 A. C. Crombie: Robert Grosseteste And the Origins of Experimental Science. 1100-1700, Oxford 1953.

5 Vgl. Andreas Speer: Scientia demonstrativa et universaliter ars faciens scire. Zur methodischen Grundlegung einer Wissenschaft von der Natur durch Robert Grosseste. In. Erfahrung und Beweis. Experience and Demonstration, hrsg. v. Alexander Fidora u. Matthias Lutz-Bachmann, Berlin 2007, S. 25-40.

6 Allerdings ist ebenfalls festzustellen, dass sich Grossetestes Ausführungen zum experimentum durchaus im Rahmen des aristotelischen Erfahrungsbegriffs bewegen. Vgl. Jürgen Sarnowsky: »Expertus - experientia - experimentum. Neue Wege der wissenschaftlichen Erkenntnis im Spätmittelalter«, Das Mittelalter 17, 2: 47-59, doi: https:// doi.org/10.1524/mial.2012.0021.

7 Jeremiah Hackett: Robert Grosseteste and Roger Bacon on the Posterior Analytics. In: Pia Antolic-Piper/Alexander Fidora/Matthias Lutz-Bachmann (Hrsg.): Erkenntnis und Wissenschaft/Knowledge and Science: Probleme der Epistemologie in der Philosophie des Mittelalters/ Problems of Epistemology in Medieval Philosophy, Berlin 2004, S. 161-212, hier S. 166. 
Neuzeit für den Prozess der Mathematisierung von scientia entscheidend werden sollten.

Schauen wir uns die beiden Beispiele - das politische und das geometrische - im Vergleich an, dann fällt das neue Moment, das in der Mathematisierung liegt, sofort ins Auge: Das angegebene Verfahren der Konstruktion lässt sich als Beweis der Aussage verwenden, dass dieses Dreieck gleichseitig ist (»Also ist das Dreieck ABC gleichseitig.»). Der Beweis ist aber zugleich eine präskriptive Anleitung, ein Rezept: durch das vorgeführte Verfahren lassen sich gleichseitige Dreiecke herstellen. Und dass sie sich herstellen lassen, hat einen einfachen Grund: die Längen-, Lagen- und Formverhältnisse sind allesamt reine Produkte von Regelverhältnissen aus den verwendeten Definitionen, Postulaten und Denkvoraussetzungen. Man spricht hier häufig davon, dass die Mathematik mit idealisierten Entitäten arbeite, aber der Kern der Sache ist nicht einmal, dass es auf die reale (immer imperfekte) Zeichnung gar nicht ankommt, sondern dass sie mit Elementen arbeitet, die selbst vollkommen durch Regeln bestimmt sind, sich also in durchgängigen Regelverhältnissen bewegt. So schließt sich der mathematische Beweis gegenüber der Wirklichkeit, während sich der logische Beweis zur Wirklichkeit hin öffnet, indem er eine Regelform des Wirklichen auszudrücken sucht.

\section{$\checkmark$ VERTIEFEND Mathematik versus Logik}

Die Frage nach dem Verhältnis von Mathematik und Logik ist ein Problem, das die Philosophie seit langem beunruhigt. Spätestens seit dem frühen 17. Jahrhundert, seit Descartes und Galilei, hatte sich ein Zweig der Philosophie mit dem Prozedere des mathematischen Operierens eng verbunden. In deren Folgeketten schien die Notwendigkeit der Ableitungen gewährleistet, dadurch sicheres Wissen ermöglicht. Dass die Folgerichtigkeit des mathematischen Vorgehens und die Folgerichtigkeit des logischen Denkens einer weltbezogenen Sprache sich unterscheiden, kam dabei aber kaum in den Blick. Das 20. Jahrhundert war beherrscht von philosophischen Schulen, die mit einem logischen Aufbau der Sprache das leisten und absichern wollten, was mathematische Verfahren in den Wissenschaften leisten. Tatsächlich ist der Unterschied zwischen den mathematischen Verfahren und dem logischen Denken nicht leicht zu fassen. Ein Aspekt dieses Unterschieds lässt sich beleuchten, wenn wir die Rolle des Präskriptiven - also des Rezepthaften - weiter ausleuchten. Beweise zeigen Folgeketten. Sie zeigen aber nicht, was de facto woraus folgte, sondern: 


\section{BASISWISSEN 5: Beweise - Zum Verhältnis von Logik und Mathematik}

Schauen wir uns dieses Verhältnis unter der Lupe an: Die drei Elemente der Ausgangsstellung, wie sie in der Zweiten Analytik kombiniert wird, führen notwendig zu bestimmten Schlüssen. Wie sieht die Ausgangsstellung in der Geometrie, in den euklidischen Elementen aus? Auch hier finden wir eine Dreiteilung der Voraussetzungen:

1. horoi (Definitionen): z.B.: Eine Linie hat eine Länge, aber keine Breite.

2. aitemata (Postulate):z.B.:Eine gerade Strecke ist beliebigverlängerbar.

3. koinai ennoiai (gemeinsame Denkvoraussetzungen): z.B.: Gleichem das Gleiche hinzugefügt ergibt Gleiches.

Die beiden griechischen Wörter axioma und aitemata sind in ihren Ursprüngen fast gleichbedeutend: es sind allgemeine Prinzipien, deren $\mathrm{Zu}$ stimmungsfähigkeit vorausgesetzt wird. Der dritte Punkt bei Euklid entspricht den grundsätzlichsten solcher Prinzipien, die von vielen Interpreten als Axiome im aristotelischen Sinne verstanden werden. Ob das Wort Axiom bei Aristoteles nun aber die jeweiligen oder aber die grundsätzlichsten Prinzipien meint - jedenfalls finden sich bei Euklid zwei andere sprachliche Ausdrücke, die in der Überlieferung beide als Reformulierung für Axiom aufgefasst wurden. So viel zu den begriffsgeschichtlichen Verwicklungen. Von der Sache her zeigt sich nun aber etwas, das von weit größerer Tragweite ist, wenn wir uns nun einem Euklidischen Beispiel zuwenden, und zwar sogleich dem ersten Beweis in den Elementen:

»Der Beweis zeigt uns, was herauskommen soll«. So formuliert es der Philosoph, der sich sicherlich am tiefgründigsten mit Beweisen auseinandergesetzt hat, Ludwig Wittgenstein, in seinen Bemerkungen über die Grundlagen der Mathematik. Und er erklärt: »da jede Reproduktion des Beweises das Nämliche demonstrieren muss, so muss sie einerseits also das Resultat automatisch reproduzieren, anderseits aber auch den Zwang, es zu erhalten. ${ }^{8}$ Das

8 Ludwig Wittgenstein: Bemerkungen über die Grundlagen der Mathematik, Werkausgabe Bd. 6, Frankfurt a.M. 1984, S. 187. 
»\$ 1 (A. 1) Über einer gegebenen Strecke ein gleichseitiges Dreieck [zu] errichten.

Die gegebene Strecke sei $A B$. Man soll über der Strecke $A B$ ein gleichseitiges Dreieck errichten.

Mit A als Mittelpunkt und $\mathrm{AB}$ als Abstand zeichne man den Kreis B C D , ebenso mit B als Mittelpunkt und B A als Abstand den Kreis A C E; ferner ziehe man vom Punkte $C$, in dem die Kreise einander schneiden, nach den Punkten A, B die Strecken C A, C B (Post. 1)

Da Punkt A Mittelpunkt des Kreises C D B ist, ist A C =A B (I, Def. 15); ebenso ist, da Punkt B Mittelpunkt des Kreises C A E ist, B C = B A. Wie oben bewiesen, ist auch $C A=A B$; also sind $C A$ und $C B$ beide $=A B$. Was aber demselben gleich ist, ist auch einander gleich (Ax. 1); also ist auch $\mathrm{C} \mathrm{A}=\mathrm{C}$ B; also sind C A, A B, B C alle drei einander gleich.

Also ist das Dreieck A B C gleichseitig (I, Def. 20); und es ist über der gegebenen Strecke A B errichtet - dies hatte man ausführen sollen.«

Euklid: Die Elemente. Buch I-VIII. Nach Heibergs Text aus dem Griechischen übersetzt und herausgegeben von Clemens Thaer, Darmstadt 1973, S. 3-4., Abb.1.

Zwingende im Beweisen kommt aber auf unterschiedliche Weise zustande, im mathematischen Beweisen anders als im logischen. Das zeigt sich, wenn wir die Analogie zu Rezepten ausbuchstabieren:

In einem sehr lockeren Sinne ist jedes Rezept, nach dem etwas hergestellt wird, ein Beweis. Denn wenn jemand nach einem Rezept, das heißt nach Vorgaben zu Zutaten und Angaben zum Vorgehen, etwa eine SacherTorte backt, ist zugleich bewiesen, dass eben diese Zutaten und Prozesse folglich das Ergebnis einer Sacher-Torte herbeiführen, sollte diese gelingen. Das Besondere des mathematischen Beweises liegt nun in folgendem Unter- 
schied: Im Fall des Kochens und Backens wird etwas NACH Rezept, nach einer Vorschrift hergestellt: auf der einen Seite steht das Rezeptbuch, auf der anderen die Zutaten und Instrumente (dazwischen eine mehr oder weniger ausgeprägte Geschicklichkeit). In der mathematischen Konstruktion ist es anders: dabei wird nicht NACH einem Regelwerk gekocht, gebacken etc., hier wird IM Regelwerk selbst konstruiert; es ist, als würde man im Rezept selbst backen; als würde man nicht Eier, Zucker, Mehl handhaben, sondern die Buchstaben, Worte und Sätze der Rezeptur zusammenstellen, umformen, erweitern - bis eine andere Gestalt entsteht, deren Eigenschaften sich schlüssig aus den Eigenschaften der eingesetzten Elemente ergibt: z.B. Gleichseitigkeit eines Dreiecks aus der gleichen Länge der eingesetzten Strecken. Die Mathematik ist Regelwerk - in seiner Anwendung auf sich selbst. Weil Mathematik stets im eigenen Medium operiert, ist jede Konstruktion zugleich ein Beweis: es lässt sich nämlich durch zeichenhafte Operationen schlüssig eine infrage stehende Struktureigenschaft auf bekannte Struktureigenschaften zurückführen, indem aus den bekannten die infrage stehende durch Konstruktion hergestellt wird.

Im Backen dagegen liegt das Gelingen des Kuchens noch an etwas ganz anderem als im Einhalten der Regeln des Rezepts: nämlich an den Eigenschaften und dem Verhalten der eingesetzten Zutaten und Hilfsmittel - und wer je gebacken hat, weiß, wieviel dabei auf oft recht undurchschaubare Weise misslingen kann. Dies ist kein gradueller, sondern - praktisch betrachtet - ein kategorialer Unterschied: das Eine ist ein Operieren in einem Regelsystem, das Andere die Anwendung des Regelsystems auf eine andere Sphäre, auf einen Realitätsbereich eigener Art, mit allen Schwierigkeiten, die das Anwenden mit sich bringen kann.

Genau in diesem Sinne unterscheidet sich nun der syllogistische, aristotelische Beweis vom mathematischen. Denn das Wissen um die Kriegsentstehung ist ein realitätsbezogenes Wissen. Die logische Form zeigt Regelzusammenhänge auf, doch diese Regelzusammenhänge stehen in einem Verhältnis zu einer äußeren Realität, an der sie sich bewähren müssen. Man kann hier fragen: trifft es überhaupt zu, dass ..., unter welchen Bedingungen trifft zu, dass ... etc. Die aristotelische apodeixis weist logische Implikationen auf, die wahr sind unter der Bedingung, dass die Prinzipien, aus denen bewiesen wird, wahr sind. Ob diese wahr sind und ob sie als Axiome vorausgesetzt werden können, muss aber unabhängig, durch Erfahrung und Einsicht gewonnen werden und bemisst sich an dieser (siehe unten: Basiswissen Induktion). 
Das ist anders im mathematischen Beweisen: Definitionen, Postulate und allgemeine Regeln bilden hier ein vollständiges und in sich geschlossenes System, das sich von jeder äußeren Realität abzukoppeln vermag. Weil der geometrische Beweis mit abstrakten Eigenschaften operiert, die auf keine Gegenstandssphäre beschränkt sind, kann auch keine Gegenständlichkeit ihre je eigenen Bedingungen und Tendenzen ins Spiel bringen. Genau durch diese Abkopplung kann er zu einem operativen System werden, ${ }^{9}$ in dem aus Elementen und Konstellationen immer weitere Elemente und Konstellationen erschlossen werden können, ohne dass sich dies an etwas anderem bewähren müsste als an innerer Folgerichtigkeit.

Nur in vollständig formalen Zeichensprachen wie den mathematischen lassen sich Systeme von Strukturen und Regeln entfalten, die völlig unabhängig davon sind, was an spezifischen Zusammenhängen in der Wirklichkeit besteht oder nicht. Das bedeutet nicht, dass mathematisches Operieren ein bloßes Spiel ist; denn die abstrakten Größen- und Mengenverhältnisse sind Eigenschaften, die wir der Wirklichkeit entnehmen und die wir teils recht erfolgreich auf Wirklichkeit applizieren können. ${ }^{10}$ Doch innerhalb des mathematischen Operierens spielt dieser Bezug keine Rolle; die Systematizität dieses Operierens resultiert gerade aus der Autonomie, also daraus, dass die Operationen mit Zeichen keiner Wirklichkeit verpflichtet ist, außer ihrer eigenen.

Der hier deutlich werdende Unterschied hat sich - kaum merklich - in unterschiedliche Konzepte von Wissenschaft eingeschlichen. Ein Aspekt der mathematischen Beweislehre als Konstruktionslehre ist, dass sich die mathematische Konstruktion als eine eigene Technik von Strukturherstellung - als ein operativer Symbolismus - im Sinne eines ganzen Arsenals von methodischen Werkzeugen und Verfahren verstehen lässt.

Was dieser Punkt für eine bestimmte Wissenschaftsform bedeutet, hat niemand so prägnant zum Ausdruck gebracht wie der britische Wissenschaftsphilosoph Stephen Toulmin, der in seinem Buch Philosophy of Science

9 Vgl. Sybille Krämer: Symbolische Maschinen. Die Geschichte der Formalisierung im historischen Abriß, Darmstadt 1988.

10 Vgl. Torsten Wilholt: Zahl und Wirklichkeit. Eine philosophische Untersuchung überdie Anwendbarkeit der Mathematik, Paderborn 2004, S. $284 \mathrm{f}$. 
von 1953 am Beispiel des physikalischen Theorems, dass Licht sich geradlinig durch den Raum bewegt, erläutert:

»Entscheidende Entdeckungen in den Naturwissenschaften bestehen im Kern aus der Entdeckung neuer Darstellungsmethoden und Folgerungstechniken, mit deren Hilfe man Schlüsse ziehen kann - und zwar auf eine Weise, die sich den untersuchten Phänomenen anpaßt. « ${ }^{11}$

Diesen Zugang, „die Phänomene auf eine neue Weise zu sehen und neue Darstellungstechniken auf sie anzuwenden«, unterscheidet Toulmin kategorisch vom »Aufstellen empirischer Verallgemeinerungen ${ }^{12}$ Als empirische Verallgemeinerungen sind Prinzipien wie das Geradlinigkeitsprinzip von Licht »einfach falsch «; ${ }^{13}$ sie haben in den Wissenschaften tatsächlich eine ganz andere Funktion:

»wenn man sie akzeptiert, gibt man zu erkennen, dass man sich die Erklärungstechniken der geometrischen Optik zu eigen gemacht hat, nämlich das Modell, nach dem das Licht von der Quelle ausgeht und sich bis zu den beleuchteten Gegenständen durch den Raum bewegt, und die Verwendung geometrischer Diagramme, aus denen man erschließt, welche Phänomene unter gegebenen Umständen zu erwarten sind. $^{14}$

Das Akzeptieren, Einüben und Denken in einer bestimmten symbolischen Darstellungstechnik ist nicht auf die geometrische Optik beschränkt, vielmehr ist sie für Toulmin ein elementarer Charakterzug moderner Wissenschaft. Bezugnehmend auf das abgebildete Diagramm der geometrischen Optik schreibt er: »Natürlich ist es nicht besonders wichtig, ob unser Physiker sein Diagramm genau so zeichnet, wie wir es hier angenommen haben, oder ob er trigonometrische Formeln benutzt. Wesentlich ist nur, daß er bestimmte mathematische Formeln oder eine andere Darstellungstechnik verwendet. ${ }^{15}$

11 Stephen I. Toulmin(im Orig. 1953): Einführung in die Philosophie der Wissenschaft, Göttingen 1969, S. 33.

12 Toulmin, 1969, S. 43.

13 Toulmin, 1969, S. 23.

14 Toulmin, 1969, S. 29.

15 Toulmin, 1969, S. 25. (Herv. im Orig.). 
Abb. 2: Aus Toulmin: Einführung in die Philosophie der Wissenschaft, S. 26.

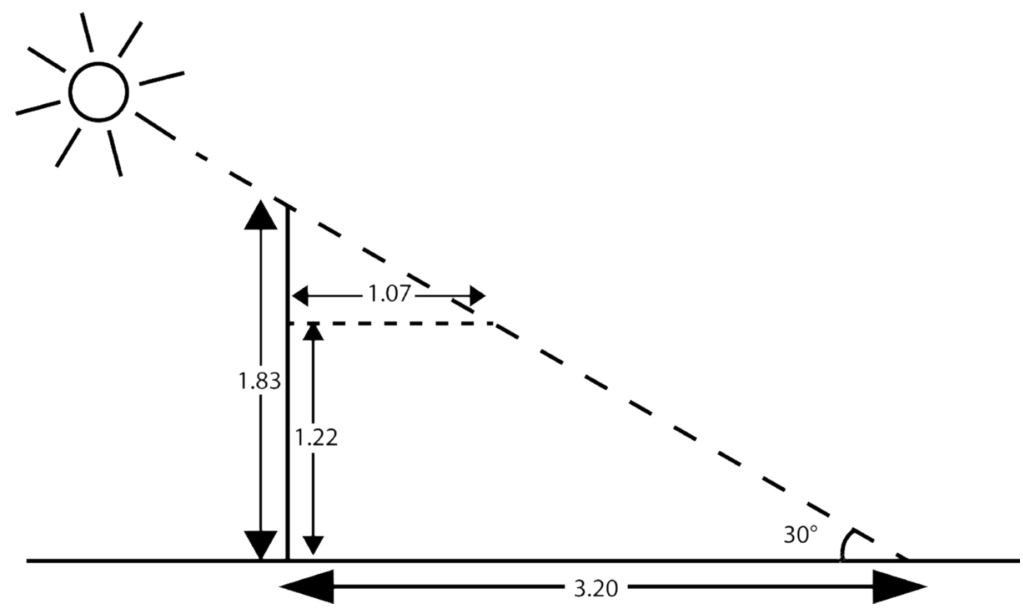

Aus Toulmins Formulierungen lassen sich zwei entscheidende Schlüsse ziehen: Erstens ist es demnach ein charakteristischer Zug zumindest einiger Wissenschaften, dass sie phänomenale Zusammenhänge in symbolischen Formalismen zur Darstellung bringen und zweitens dienen diese Formalismen nicht nur der retrospektiven Darstellung, sondern fungieren außerdem als - wie Toulmin es treffend ausdrückt - »Folgerungstechniken « ${ }^{16}$. Das heißt: Innerhalb des Symbolismus können durch Operationen regelförmiger Art strukturell neue Zusammenhänge erzeugt werden, die erst im Nachhinein mit phänomenalen Zusammenhängen rückvermittelt werden. Toulmins Beschreibung der Wissenschaften vom Typ Physik - er selbst grenzt sie gegen »naturgeschichtliche« Disziplinen $a b^{17}$ - gibt uns also ein Bild davon, welche Rolle mathematische Formalismen in den Wissenschaften spielen können. An der Frage, ob eine solche Entfaltung symbolischer Formalismen ein eigenständiger Zugang zu Erkenntnis ist oder lediglich ein Instrument in der experimentellen Forschung, scheiden sich die Geister - wie wir sogleich sehen werden. Festhalten können wir hier, dass das wirkmächtige Galileische Paradigma der Wissenschaft genau dadurch geprägt ist: das mathematische Schlussfolgern ist in dieser Konzeption der genuine Weg zu

16 Toulmin, 1969, S. 31.

17 Toulmin, 1969, S. 44. 
wahren Erkenntnissen, da nämlich die Natur selbst als eine mathematische Ordnung aufgefasst ist. Genau dies wird sich als Problem erweisen, wenn es nun um das `Schicksal der Induktion geht.

\section{Die Rolle des Experimentierens und das iSchicksal der Induktionı}

Der Status der Mathematik in den Wissenschaften ist ein viel diskutiertes Thema. Aber klar ist: die Mathematik gilt entweder als Idealbild oder als Sonderfall einer Wissenschaft, dem Normalfall entspricht sie jedenfalls nicht. Dazu fehlt ihr jenes Moment der Empirie, der Verbindung mit einem spezifischen Gegenstandsfeld, von dem sie Wissen produzieren könnte. Eine Standardauffassung besagt, dass der empirische Bezug in den Wissenschaften durch Experimente hergestellt wird. Doch die Idee, dass Wissenschaft im Kern durch Experimente gekennzeichnet ist, kommt zunächst ganz ohne Bezug zur Mathematik aus. Eine typische Formulierung lautet:

»Das richtig angestellte und richtig verstandene Experiment bildet zusammen mit der Beobachtung den Grund, von dem wir durch Induktion zur Erkenntnis der Naturgesetze gelangen. Im Altertum und Mittelalter finden wir in der Regel noch keine auf Experimente gegründete Naturerkenntnis, sondern an ihrer Stelle metaphysische Spekulationen. Erst Galilei und besonders Bacon von Verulam [Francis Bacon] schlugen den Weg der Erfahrung ein und begründeten damit eine wissenschaftliche Naturerkenntnis. ${ }^{18}$

Das Zitat stammt aus dem Eintrag »Das Experiment in den Wissenschaften" in einem aktuellen Studienbuch der Wissenschaftstheorie, das wiederum ein Konversationslexikon von 1890 zitiert und folgendermaßen kommentiert:

»Bei der entscheidenden Rolle, die hier dem Experiment bei der Erzeugung von Wissen zugesprochen wird, muss es verwundern, dass sich in dieser Zeit in der Wissenschaftsphilosophie eine deutlich andere Auffassung als die zitierte durchsetzt und insgesamt das Interesse am Experiment stark abge-

18 Michael Heidelberger: Das Experiment und die Wissenschaften. In: Wissenschaftstheorie. Ein Studienbuch, Paderborn 2007, S. 155-176, hier S. 155. 
nommen hat. Mit der Umdeutung des Experiments in der philosophischen Diskussion [...] verlagerte sich der Schwerpunkt auf den Begriff der Theorie und ihre Funktion. ${ }^{19}$

Die Überzeugung, dass wissenschaftliche Erfahrung ein Weg des Experimentierens ist, ist auf eigentümliche Weise zugleich selbstverständlich und alles andere als selbstverständlich. Doch die Ambivalenz, von der hier die Rede ist, ist schon viel älter: sie lässt sich zurückverfolgen bis zu einer Wendung, die über Vorstufen in der Renaissance, etwa bei Leonardo da Vinci, schließlich Galilei und Descartes der Sache gaben - wovon auch der Begriff der Induktion betroffen war.

Wo befinden wir uns? 1620 erschien Francis Bacons Werk Novum Organum - ein großangelegter Versuch, die aristotelische Lehre in eine Experimentaltheorie zu überführen; 1623 Galileo Galileis Il Saggiatore - die Goldwaage, 1632 sein Dialog über die beiden hauptsächlichen Weltsysteme, 1637 Descartes Discours de la méthode und 1644 seine Principia philosophiae. Innerhalb von gut 20 Jahren sind also eine ganze Reihe grundlegender Arbeiten zum Verhältnis von Wissenschaft und Experiment erschienen. Bemerkenswerterweise findet in dieser Reihe aber eine fundamentale Umdeutung statt. Denn während Francis Bacon das Experiment zur eigentlichen Methode wissenschaftlicher Erkenntnis erhebt, degradieren es Galilei und Descartes zu einem Instrument, durch das Erkenntnisse, die auf ganz anderem Wege gewonnen werden, nur noch geprüft werden.

Bacon prägt den lateinischen Begriff experimentum so, dass solche Erfahrungen, die "geschickt und kunstvoll ausgedacht und angewendet werden $«,{ }^{20}$ die einzig geeigneten Mittel sind, allgemeine Prinzipien der Natur nicht nur vorauszusetzen, sondern allererst herzuleiten. Dabei wendet er sich ausdrücklich gegen eine Verkürzung von Wissenschaft auf Beweislehre (»[d]iese Arbeit, Untersuchung und Durchwanderung der Welt kann durch keinen Scharfsinn, kein Nachdenken und keine Beweisführung ersetzt oder ausgeglichen werden ${ }^{21}$ ) und gegen eine Verkürzung auf Naturgeschichte (»die Beobachtung ist nachlässig, unregelmäßig und gleichsam zufällig [...] die Naturgeschichte [...] ist leichtfertig und armselig - alle diese haben den

19 Heidelberger, 2007, S. 155.

20 Francis Bacon: Neues Organon, Hamburg 1999, S. 49.

21 Bacon, 1999, S. 53. 
Geist nur mit dem mangelhaftesten Stoff für die Philosophie und Naturwissenschaft [hier steht im Orig. nur »scientia«] versorgt.«). ${ }^{22}$ Bacon konzipiert dagegen eine wissenschaftliche Methode, die einen Mittelweg zwischen sammelnder Empirie und konstruktiver Rationalität einschlägt. ${ }^{23}$ Das Experimentieren beruht auf der Verknüpfung von beidem: es ist eine Interaktion zwischen Überlegung zur Einrichtung der Erfahrung und der Erfahrung, die sich daraus am Material ergibt.

Ganz anders Galilei und Descartes: Beide sind - in diesem Punkt wesentlich konservativer - dem Ideal der Beweisführung, wie es sich seit dem 12. Jahrhundert ausprägte, verpflichtet, und zwar in seiner mathematisch transformierten Form. Wissenschaft ist für beide zunächst formale Ableitung: Da die »Wahrheit, deren Erkenntnis durch die mathematischen Beweise vermittelt wird, dieselbe ist, welche die göttliche Weisheit erkennt «, ${ }^{24}$ könne, so Galilei, das menschliche Erkennen zwar nicht wie das göttliche auf einen Blick, aber doch »schrittweise von Schluss zu Schluss ${ }^{25}$ voranschreiten und durch »Folgerungen ..., welche streng bewiesen sind ${ }^{26}{ }^{26} \mathrm{zur}$ Wahrheit gelangen. Und auch für Descartes stellen »[j]ene langen Ketten ganz einfacher und leichter Begründungen, die die Geometer zu gebrauchen pflegen, um ihre schwierigsten Beweise durchzuführen« das Ideal wissenschaftlicher Methode dar. Das Experiment dagegen tritt für beide erst auf den Plan, wenn dieser Erkenntnisweg abgeschlossen ist: Galilei bestimmt die Rolle des Experiments als die einer zusätzlichen Bestätigung »in den Wissensgebieten, in welchen auf natürliche Consequenzen mathematische Beweise angewandt werden; so sieht man es bei Allen, die Perspective, Astronomie, Mechanik, Musik und Anderes betreiben; diese alle erhärten ihre Prinzipien durch Experimente «. ${ }^{27}$ Ebenso überlässt Descartes dem Experiment lediglich die Rolle des Entscheiders über verschiedene rational erschlossene Möglichkeiten: In der Schwierigkeit nämlich, dass »der Bereich des von Na-

\footnotetext{
22 Bacon, 1999, S. 53.

23 Vgl. Bacon, 1999, S. 211.

24 Galilei, Galileo: Dialog über die Weltsysteme. In: ders., hrsg. u. eingel. v. Hans Blumenberg: Sidereus Nuncius. Nachricht von neuen Sternen, Frankfurt a.M. 1965, S. 157

25 Galilei, 1965, S. 157.

26 Galilei, 1965, S. 158.

27 Galileo Galilei: Unterredungen und mathematische Demonstrationen über zwei neue Wissenszweige, die Mechanik und die Fallgesetze betreffend, Erster bis sechster Tag, hrsg. von Arthur Oettingen, Darmstadt 1964, S. 162.
} 
tur Möglichen so groß und so weit ist und daß diese Prinzipien so einfach und allgemein sind, daß ich fast keine besondere Wirkung mehr beobachte, von der ich nicht gleich anfangs erkenne, daß sie auf mehrere verschiedene Weisen davon abgeleitet werden kann ${ }^{28}{ }^{28}$ weiß Descartes »keinen anderen Ausweg als wiederum etliche Beobachtungen anzustellen [chercher ... quelques expériences; WK.] und zwar solche, die anders ausfallen, je nachdem die eine oder die andere Erklärungsart richtig ist. $\aleph^{29}$

Damit wurde der Nachwelt eine wenig beachtete, aber umso virulentere Frage übermittelt: Sind Experimente nur der Prüfstein für Erkenntnisse, die durch mathematische Demonstrationen gewonnen werden oder bilden sie das eigentliche Zentrum von Wissenschaft? Diese Frage scheidet in der Folgezeit die Geister. Auf der einen Seite steht die Wissenschaftsphilosophie, die in der Tradition Galileis und Descartes in weiten Teilen eine Standardauffassung zugrunde legt, die so vertraut ist, dass sie als Gemeinplatz erscheint. So liest man etwa in einem populärwissenschaftlichen Magazin Autor ist ein Wissenschaftsphilosoph:

»Im Cegensatz zu seinem antiken Vorgänger ist der moderne Atomismus echte Naturwissenschaft. Er formuliert Cesetze, mit deren Hilfe man das Verhalten der Teilchen berechnen und diese Berechnungen experimentell überprüfen kann. «30 (Herv. WK.)

Ausgangspunkt sind Gesetze - die ideengeschichtlichen Erben der Axiome und Prinzipien ${ }^{31}$-; aus ihnen wird rechnerisch konstruiert und, wenn so ein bestimmtes Verhalten gefolgert wurde, wird im Nachhinein noch experimentell geprüft. Bacons Erkenntnisweg war genau umgekehrt. Aus der Arbeit am Material und der experimentellen Erzeugung bestimmter fraglicher Zustände werden Annahmen sukzessive verfeinert, bis schließlich ein gut fundiertes Verständnis der zugrundeliegenden Prinzipien und Gesetze sich herausschält.

28 René Descartes: Discours de la méthode, Hamburg 1990, S. 105.

29 Descartes, 1990, S. 107.

30 Michael Esfeld: Wissenschaft, Erkenntnis und ihre Grenzen. In: Spektrum der Wissenschaft 8, (2017), S. 12-18, hier S. 14.

31 Michael Hampe: Eine kleine Geschichte des Naturgesetzbegriffs, Frankfurt a.M. 2007, S. 64ff. 


\section{BASISWISSEN 6: Induktion und Empirie - Der Weg der Erfahrung}

Im Kontext der Diskussion um die verschiedenen Rollen des Experimentierens steht auch das >Schicksal der Induktion<. Der Widerstreit zwischen den unterschiedlichen Auffassungen von Erfahrung und Experiment hat nämlich auch diesen Begriff, der so grundlegend in der Wissenschaftstheorie ist, einbezogen und zu Verwirrungen geführt, die wir in groben Umrissen an dieser Stelle klären sollten.

Erstens ist das Wort inductio die lateinische Übersetzung der griechischen Vokabel epagogé. Epagogé ist ein zentraler Begriff der aristotelischen Erkenntnislehre. Er meint die Herausbildung allgemeiner, auf das Ganze (katholou) bezogener Einsichten aus der Flut einzelner Wahrnehmungen, und zwar über die Stationen Erinnerung und Erkennen von Ähnlichkeiten. Diese Kompetenz, in einem Komplex von Fällen relevante Ähnlichkeiten zu sehen, nennt Aristoteles, Erfahrung (empeíria). Epagogé ist daher bei Aristoteles ein im ursprünglichen Sinne sempirischer Erkenntnisweg, auf dem sich Menschen in ihrem Erkenntnisstreben gleichsam naturwüchsig bewegen. Als Weg zu Erfahrenheit und Einsicht (bei Aristoteles: nous), entsteht ein allgemeines Wissen, das schon empirisch gegeben ist, wenn es durch logische Schlussfolgen (syllogismos) und Beweisverfahren (apodeixis) expliziert wird. Epagogé ist deshalb bei Aristoteles Voraussetzung für alle logisch-formalen Erkenntnisprozesse.

Der Grundgedanke einer dem Beweisen vorgeschalteten Erfahrung blieb als Bezugspunkt zum Ideal der scientia im Hochmittelalter und in der frühen Neuzeit stets erhalten. Ausdrücke für diesen Zusammenhang, nämlich«via experientiae und via rationis oder ratio et experimentum (Köhler 129-133) waren stehende Wendungen. Sämtliche die Debatte prägenden Autoren der Zeit wie Robert Grosseteste, Albert Magnus, Thomas von Aquin oder Roger Bacon rezitierten regelmäßig die aristotelischen Formulierungen zur Erfahrungsbildung, die jeder Beweisführung vorausging. Und auch noch das Novum Organum des Francis Bacons kann als Wiederbelebung dieser aristotelischen Konzeptionen gegen die sich allmählich ausbreitende szientifische Überformung, gegen ihre Logifizierung gelesen werden. 
Diese Tendenz der Logifizierung machte nämlich aus der empirischen aristotelischen Induktion ein Schlussverfahren: Im Zuge der Herausbildung eines Wissenschaftsideals, das sich am Begriff der episteme orientierte, konnte es so erscheinen, als müsste das, was ja eigentlich Voraussetzung für die Möglichkeit logischer Beweisführung war, selbst ein auf logischer Zwangsläufigkeit beruhendes Verfahren sein. Die Verwirrungen, die durch diesen in sich widersprüchlichen Begriff einer logischen Induktion entstanden sind, wirkten bis weit ins 20. Jahrhundert nach. Die logisch-empiristische Wissenschaftsphilosophie verstrickte sich immer tiefer in das Dilemma, einerseits Wissenschaft als durchgängig begründeten Gewissheitszusammenhang sehen zu wollen, andererseits der Tatsache nicht entgehen zu können, dass noch so viele Einzelbelege einer These keine allgemeine Behauptung beweisen können. »Allgemeine wissenschaftliche Gesetze gehen immer über die endliche Menge der vorhandenen, sie unterstützenden Beobachtungen hinaus und können daher niemals in dem Sinn bewiesen werden, dass sie sich logisch aus dem Evidenten ableiten lassen.«(39) So hat es der Wissenschaftstheoretiker Alan F. Chalmers ausgedrückt und bringt damit auf den Punkt, »dass wissenschaftliche Erkenntnis nicht aus Tatsachen abgeleitet werden kann, wenn sabgeleitet als >logisch erschlossen interpretiert wird.«(37) Der Induktivismus könne deshalb nicht mit Bezug auf Logik, sondern nur mit Bezug auf Erfahrung gerechtfertigt werden (42f.) - genau dies war bereits die Einsicht, die Aristoteles zur Konzeption einer gegenüber der Syllogistik vorgängigen und radikal eigenständigen epagogé geführt hatte.

Theodor W. Köhler: Sachverhaltsbeobachtung und axiomatische Vorgaben. Zur Struktur wissenschaftlicher Erfassung konkreter Äußerungsweisen des Menschlichen im 13. Jahrhundert. In: Antolic-Piper u.a. (Hrsg.): Erkenntnis und Wissenschaft, Berlin u.a. 2004, S. 125-150. Alan F. Chalmers: Wege der Wissenschaft. Einführung in die Wissenschaftstheorie, Berlin u.a. 2001 (5. überarb. u. erw. Auflage). 
Beide Auffassungen wurden tradiert, seit Mitte des 19. Jahrhundert dominierte allerdings die Cartesianisch-Galileische Auffassung das öffentliche Bewusstsein, bis im 20. Jahrhundert sich, zuerst nur punktuell, dann auf breiter Front, die Baconsche Auffassung wieder Gehör verschaffte. Und zwar geschah dies, indem zuerst einige praktizierende Wissenschaftler ein ganz anderes Bild ihrer Tätigkeit zeichneten als es die Wissenschaftsphilosophie vorsah, ${ }^{32}$ sodann Forschungen in Wissenschaftsgeschichte und -soziologie zeigten, dass wissenschaftliche Erkenntnis in sehr anderer Weise zustande kommen kann, als dies im Galileischen Modell angenommen wurde. Diese Einsicht hat schließlich auch die Wissenschaftsphilosophie erreicht, die im Laufe ihrer Entwicklung im 20. Jahrhundert »einen immer größeren Erfahrungsbezug « ${ }^{33}$ ausbildete, bis sie schließlich ihr kanonisches Werk in Thomas S. Kuhns The Structure of Scientific Revolutions fand. Kuhn selbst hatte zwar das Experiment eher stiefmütterlich behandelt, doch sein Gedanke, dass Wissenschaften in Paradigmen, also in musterhaften Konstellationen, arbeiten, legte auch nahe, diese Muster nicht als Regelwissen, sondern als »wissenschaftliche Betätigung « ${ }^{34}$ aufzufassen (was bereits Kuhn in seinem Postskriptum von 1969 ausdrücklich tut). Aufbauend darauf entwickelte sich eine Strömung, die unter dem Titel New Experimentalism prominent wurde. Für sie ist Wissenschaft "Wissensbildung durch technisch-intervenierendes Handeln ${ }^{35}$ Die technische Seite des Experiments, die Instrumente, die materiellen Versuchsgegenstände, die Labore, die Verfahren und die Fertigkeiten der Experimentatoren wurden zum zentralen Thema. Entsprechend wurde herausgearbeitet, dass diese praktischen Welten der Experimentalsysteme ein Eigenleben ${ }^{36}$ führen, aus dem viele theoretische Fragen erst entstehen. Im Sinne eines Primats des Experimentierens haben eine ganze Reihe von Wissenschaftstheoretiker wie

32 Valer Ambrus: Vom Neopositivismus zur nachanalytischen Philosophie, Frankfurt a.M. 2002, S. 34-40.

33 Ambrus: Vom Neopositivismus zur nachanalytischen Philosophie, 2002, S. 40.

34 Thomas S. Kuhn: Die Struktur wissenschaftlicher Revolutionen, Frankfurt a.M. 1988, S. 203.

35 Michael Heidelberger: Die Erweiterung der Wirklichkeit im Experiment. In: Micheal Heidelberger/Friedrich Steinle (Hrsg.): Experimental Essays = Versuche zum Experiment, Baden Baden 1998, S. 11.

36 Ian Hacking: Do Thought Experiments Have a Life of Their Own? Comments on James Brown, Nancy Nersessian and David Cooding. In: PSA: Proceedings of the Biennal Meeting of the Philosophy of Science Association. Vol. Two: Symposia and Invited Papers, 1992, S. 302-308. 
Ian Hacking, ${ }^{37}$ Holm Tetens, ${ }^{38}$ Bruno Latour, ${ }^{39}$ Andrew Pickering, ${ }^{40}$ Don Ihde, ${ }^{41}$ Hans-Jörg Rheinberger ${ }^{42}$ und Joseph Rouse ${ }^{43}$ die spezifische Praxisform des Experimentierens herausgestrichen und deutlich gemacht, dass es für forschungsförmiges, also auf neue Erkenntnis gerichtetes, Experimentieren charakteristisch ist, einen ergebnisoffenen Prozess auszutragen ${ }^{44}$ - und gerade nicht nur vorgefasste theoretische Möglichkeiten zu prüfen.

37 Ian Hacking (Orig. 1983 (Representing and Intervening)): Einführung in die Philosophie der Naturwissenschaften, Stuttgart 1996.

38 Holm Tetens: Experimentelle Erfahrung, Hamburg 1987.

39 Bruno Latour: Science in Action: How to Follow Scientists and Engineers through Society, Cambridge MA 1987.

40 Andrew Pickering: The Mangle of Practice: Time, Agency, and Science, Chicago 1995.

41 Don Ihde: Instrumental Realism: The Interface between Philosophy of Science and Philosophy of Technology, Bloomington/Indianapolis 1991.

42 Hans-Jörg Rheinberger: Experiment. Differenz. Schrift: Zur Ceschichte epistemischer Dinge, Marburg an der Lahn 1992; Hans-Jörg Rheinberger: Experimentalsysteme und epistemische Dinge. Eine Ceschichte der Proteinsynthese im Reagenzglas, Cöttingen 2001.

43 Joseph Rouse: Engaging Science: How to Understand its Practices Philosophically, Ithaca/ Cornell 1996.

44 Bruno Latour betont in Bezug auf das Experimentieren: »es hat etwas Neues, von der Veranstaltung Unabhängiges herauszukommen, sonst ist die ganze Unternehmung vertan« (Bruno Latour: The force and the reason of experiment. In: H. E. Le Grand (Hrsg.): Experimental Inquieries, Dodrecht 1990, S. 49-80, hier: S. 64. (Übersetzt in Hans Jörg Rheinberger: Experiment. Differenz. Schrift: Zur Geschichte epistemischer Dinge. Marburg an der Lahn 1992, S. 16.); Hans-Jörg Rheinberger schreibt: »Wenn danach gefragt wird, was den Forschungsprozeß treibt, ist es ratsam, mit einer Charakterisierung der Experimentalsysteme, ihrer Struktur und ihrer Dynamik zu beginnen, und nicht von einem ursprünglichen, unhintergehbaren, wie auch immer formulierten Primat der Theorie. [...] Was zur Debatte steht, ist ... eine durch instrumentelle Randbedingungen ausgerichtete Bewegung, in der das Räsonnieren gewißermaßen ins Spiel der materiellen Entitäten gerissen wird." (Rheinberger 1992, S. 22). Die Bestimmung des Wissenschaftsobjekts im experimentellen Prozess beschreibt Rheinberger so: »Man verfehlt seine Spezifik, wenn man diesen Vorgang als den der stheoretischen< Abbildung einer wie immer gearteten ,Wirklichkeit betrachtet. Was praktisch im Forschungsprozess abläuft, ist die Realisierung, also Produktionvon Wissenschaftsobjekten mitHilfevon Dingen, die bereits als hinreichend stabile Materialformen von Wissen betrachtet und gehandhabt werden können. [...] Dieser Prozeß ist keineswegs von vornherein zielgerichtet. Er muß durch jene Vorgänge ertastet werden, die [François] Jacob beschrieben hat als sdie gescheiterten Anläufe, die verfehlten Experimente, die Stottereien, die blödsinnige Probierereien<. Letztlich bleibt der einzige Führer durch diese Landschaft, wie Goethe gesagt haben würde, ১die Verfahrensart selbst.، Sie allein produziert die Hinweise, in welche Richtung man sich zu wenden und wo man 
So zeigen sich auch innerhalb der Experimentaltheorie zwei grundsätzlich verschiedene Auffassungen von Wissenschaft. Was ist Wissenschaft? Ein gedankliches Operieren, eine intellektuelle Tätigkeit, die Kraft logischer Schlussfolgerung und mathematischer Berechnung zu Ergebnissen kommt, die sich in Experimenten prüfen lassen - wie bei Sherlock Holmes die >Theorieく im Geständnis des Delinquenten. Oder ist Wissenschaft das, was sich am deutlichsten in Laboren zeigt, nämlich der Versuch, die Bedingungen von Prozessen in den Griff zu bekommen, sodass sie systematisch variiert und regelhafte Verläufe beobachtet werden können - Experimentalsysteme, bei denen die Tücke stets im Detail liegt und die eine hoch spezialisierte Ausbildung, Erfahrung und Könnerschaft verlangen, um die Versuchsanordnungen so weit zu stabilisieren, dass signifikante und valide Ergebnisse gewonnen werden können?

\section{Laborwissenschaft, Modellierung, Simulation und Realexperimenter}

\section{Was macht die Wissenschaft im Labor?}

Die Antwort lautet: sie operiert mit Modellen an Modellen. Und zwar operiert sie mit physischen Modellen an symbolisch-operativen (häufig mathematischen, aber auch anderen Formelsprachen) und mit symbolisch-operativen an physischen Modellen. Das Verhalten von Wellen, Blitzen, Stadtflüchtigen oder Viren lässt sich auch in der offenen >Natur < beobachten - es ins Labor zu holen, bedeutet vor allem eines: es von der Umwelt abzutrennen, es als Komplex zu isolieren und kontrollierbar, kurz: es zum Modell zu machen. Laborbedingungen einzurichten bedeutet, die physischen Prozesse so zu präparieren, dass einzelne Bedingungen gezielt variiert werden können. Genau dadurch lässt sich das Verhalten der Forschungsgegenstände in Formeln ausdrücken, deren Umformung Prognosen erlaubt: die chemische Reaktionsgleichung zeigt nicht nur, wie sich Substanzen verhalten haben, sondern auch wie sie sich verhalten werden (- unter Laborbedingungen - wenn

umzukehren hat.«(Rheinberger 1992, S. 29f.). Zu erwähnen ist hier auch, dass diese Autoren damit die klassisch moderne Position, die etwa Liebig gegen Bacon vertritt, revidieren und in mancherlei Hinsicht zum Baconschen Ansatz zurückkehren. 
alles funktioniert; dazu später mehr). Umgekehrt werden die Formelsysteme den beobachteten $>$ Naturprozessen $<$ so angepasst und weiterentwickelt, dass sie dem Verhalten der Forschungsgegenstände immer besser entsprechen: der Weg der Formelsprachen und der mathematischen Modellierung.

Das typische Labor ist also ein doppeltes Labor: einerseits arbeitet es im Medium der symbolisch-operativen Sprache, andererseits im physischen Medium des Forschungsgegenstandes. Ein Beispiel: um Zellprozesse zu verstehen, versucht man, elementare Prozesse der lebenden Zelle im Labor zu reproduzieren und zugleich diese Erkenntnisse in ein Computermodell der Zelle zu integrieren - »bis eine gute Übereinstimmung zwischen den experimentellen Daten und den modellierten Vorhersagen erreicht ist. « ${ }^{45}$

An diesem Beispiel lässt sich noch mehr zeigen: die ursprüngliche Formelsprache der Molekularbiologie ist die der Genexpression, also: wie die (in Buchstabenfolgen dargestellten) DNA-Abschnitte in (ebenfalls in Buchstabenfolgen dargestellten) Ketten der Proteine übersetzt werden. Flankiert wurde dieser Grundformelbestand durch einige Regeln der Biochemie. Je weiter aber die Einsicht in die Komplexität zellulärer Prozesse gedieh, desto größer wurden die Ansprüche an die Modellierung der Prozesse; die elementaren Formeln der Genexpression bilden zwar noch immer das theoretische Zentrum, den Kern der molekularbiologischen Forschung, doch lassen sich die Vielzahl der voneinander abhängigen und rückgekoppelten Einflussfaktoren nur noch in ebenfalls sehr komplexen und raffiniert aufgebauten Computermodellierungen reproduzieren. So versucht man, im Medium von Computerberechnungen, also in silico, herzustellen, was in der lebenden Zelle, in vivo, geschieht; ein Geschehen, das nicht unmittelbar beobachtbar ist, sondern aus Effekten in Laborversuchen in vitro (im Reagenzglas) rekonstruiert wird.

45 E. Klipp/R. Herwig/A. Kowald/C. Wierling/H. Lehrach (Hrsg.): Systems Biology in Practice. Concepts, Implementation and Application, Weinheim 2004, S. 4 [Übersetzung der Verfasser]. Das Zitat lautet im Kontext: »Systembiologie beruht auf der Integration von Experiment, Datenverarbeitung und Modellierung. Idealerweise ist dies ein sich wiederholender Prozess. Experimentell gewonnenes Wissen über das untersuchte System führt, gemeinsam mit offenen Fragen, zu einem Ausgangsmodell. Dieses Ausgangsmodell erlaubt Vorhersagen, die in neuen Experimenten verifiziert und falsifiziert werden können. Unstimmigkeiten stimulieren den nächsten Schritt der Modellentwicklung, was wiederum zu experimentell überprüfbaren Vorhersagen führt. Diese Wiederholung wird weiter geführt, bis eine gute Übereinstimmung zwischen den experimentellen Daten und den modellierten Vorhersagen erreicht ist.«. 
Am besten gelingt dieses Forschungsmodell, wo sich unter Laborbedingungen die Komplexität so weit reduzieren lässt, dass sie in symbolsystemischen Modellierungen formelhaft zu reproduzieren ist. Wie weit dies jeweils gelingen kann, ist umstritten, ${ }^{46}$ und stets ist auch die Antwort auf die weitergehende Frage offen, wie sich Ergebnisse aus dem Labor auf Verhältnisse in >freier Wildbahn übertragen lassen.

Modellierungen ohne Labor gehören ebenfalls zu diesem Forschungstyp. Im Falle der Modellierung von Klimaveränderung, Verstädterung oder einer Pandemie lassen sich die >In-vivo-Prozesse nicht oder nur sehr eingeschränkt ins Labor bringen. Die Seite der Forschung im physischen Material wird hier durch beobachtende Datensammlung realisiert - anstelle von experimenteller. Da die Modellierung hier nicht auf die kontrollierte Reproduktion physischer Prozesse zurückgreifen und diese vielmehr nur im Medium des mathematischen Modells darstellen kann, spricht man von Simulation. ${ }^{47}$

Laborwissenschaft, Modellierung und Simulation verknüpfen also experimentelle Praxis mit formaler Folgerungstechnik. Doch wer glaubt, dass diese raffinierte Erfindung eines Forschungstyps sich bruchlos als Synthese aus den älteren Paradigmen der Episteme-Scientia-Tradition ergibt, der verkennt die wissenschaftsphilosophische Sprengkraft, die in diesem Typus liegt. Denn, da Mathematik im Spiel ist, sieht es zwar so aus, als ob hier Beweisverfahren ausgeführt werden - doch tatsächlich haben die Folgerungstechniken hier eine ganz andere Funktion; und da Experimente im Spiel sind, sieht es zwar so aus, als ob Gesetzmäßigkeiten geprüft und bestätigt werden, doch tatsächlich fügt sich das reale Experimentieren ebenso wenig in dieses Bild - wie der letzte Abschnitt schon zeigte.

Die theoretische Sprengkraft dieser Ausprägung des Episteme-ScientiaTypus liegt darin begründet, dass ein Schlüsselbegriff der Wissenschaften davon betroffen ist: Naturgesetze. Naiv stellen wir uns den Zusammenhang

46 Eine Kritik aus Insider-Perspektive ist: ]. D. Sterman: A Skeptic's Guide to Computer Models. In: C. O. Barneyet al. (Hrsg.): Managing a Nation: The Microcomputer Software Catalog. Boulder, CO 1991, S. 209-229.

47 Vgl. Gabriele Gramelsberger: Simulation and Systems Understanding. In: Hanne Andersen/Dennis Dieks/Wenceslao ]. Gonzalez/Thomas Uebel/Gregory Wheeler (Hrsg.): New Challenges to Philosophy of Science, The Philosophy of Science in a European Perspective, Bd. 4, Dordrecht 2013, S. 151-161; Petra Gehring/Michael M. Resch/Andreas Kaminski (Hrsg,): The Science and Art of Simulation. Exploring - Understanding - Knowing, Heidelberg/ New York 2017. 
etwa so vor (ich zitiere hier die prägnante Formulierung der Wissenschaftsphilosophin Sandra Mitchell):

»[Laws] are what science supposedly seeks to discover. They are supposed to be the codifications of knowledge about the world that enable us to explain why what happens, happens, to predict what will happen in the future or in other circumstances and provide us the tools to intervene in the world in order to reach our pragmatic goals. ${ }^{48}$

Was Naturgesetze sind, war allerdings nur innerhalb der klassischen Beweislehre der Episteme-Scientia-Tradition klar. Hier spielen sie die Rolle von universal gültigen Prinzipien, die entdeckt werden können und aus denen sich - durch Folgerungstechnik abgeleitet und durch Experimente bestätigt, Erklärungen und Vorhersagen erstellen lassen. Doch je genauer man auf die Realität der Forschung blickt, desto unklarer wird auch der Begriff des Naturgesetzes. So kommt C. A. Hooker im Eintrag zu »Laws, natural« der Routledge Encyclopedia of Philosophy zu der Konklusion:

»The existence and nature of laws is a complex problem. [...] There is no uncontroversial theory of laws: all face difficulties. As fast as we scientifically unravel nature's mysteries, so fast does the nature of that understanding become mysterious. ${ }^{49}$

Ein knappes Jahrzehnt später hat sich an dieser Sachlage offensichtlich wenig geändert. Andreas Hüttemann resümiert: »Eine restlos überzeugende Theorie der Naturgesetze gibt es nicht. $\aleph^{50}$

48 Sandra D. Mitchell: Ceteris Paribus: An Inadequate Representation for Biological Contingency. In: Erkenntnis. An International Journal of Analytic Philosophy, 57(2002), S. 329-350, hier S. 329.

49 Routledge Encyclopedia of Philosophy (1998): Eintrag »Laws, natural«. Vol. 5, S. 474.

50 Andreas Hüttemann: Naturgesetze. In: Andreas Bartels/Manfred, Stöckler (Hrsg.): Wissenschaftstheorie, Paderborn 2007, S. 135-154, hier S. 153. 


\section{BASISWISSEN 7: Was sind Naturgesetze?}

Will man den Naturgesetzbegriff verstehen, so muss man sich zunächst klar machen, dass dieser Begriff selbst nicht naturwissenschaftlich begründet ist. In den Formulierungen von Gesetzen der Bewegung, Kraft, Optik oder Wärmelehre kommt der Gesetzesbegriff selbst nicht vor. (Vgl. Hampe 46; Vollmer) Diese Formulierungen werden als Gesetze betrachtet, eine naturgesetzliche Definition des Gesetzesbegriffs gibt es aber nicht.

In der Begriffswelt der griechischen Antike bilden Natur (physis) und Gesetz (nomos) zunächst ein Gegensatzpaar. Natur als das, was von selbst entsteht, vergeht und sich bewegt, steht im Gegensatz zu den Gesetzen, die Menschen erlassen und einrichten. Ausdrücklich metaphorisch konnte zwar auch schon in der klassischen Antike die Natur als gleichsam gesetzlich geordnet beschrieben werden, doch durch solche rhetorischen Wendungen wurde der Gegensatz nicht aufgehoben, sondern in Gebrauch genommen. Das änderte sich erst mit der Stoa und schließlich mit dem Konzept eines christlichen Schöpfergottes, der die Welt als eine gesetzmäßige Ordnung erschafft und einrichtet.

Die entscheidende Wendung kam aber erst mit der Etablierung der geometrischen Beweislehre in der frühen Neuzeit. Der Naturgesetzbegriff rückte nun allmählich an die Stelle des Axiomsbegriffs. Noch Kepler (und später auch Newton) sprechen von Axiomen, um die Sätze zu bezeichnen, aus denen sie Planetenbewegungen und -konstellationen geometrisch ableiten. In diesen Ableitungen kommt nun aber auch der Gesetzesbegriff zum Einsatz: »er bezeichnet vor allem diejenige Regel, die zur Beschreibung geometrischer Proportionen nötig ist«. (Hampe 66) Ob Gesetze in den natürlichen Elementen und ihren Beziehungen selbst liegen oder ob sie ihnen von außen auferlegt sind (etwa durch eine göttliche Macht), ob sie real sind oder ideelle Denkvoraussetzungen - diese Fragen sind seitdem umstritten. (vgl. Hampe 71-76) Für die moderne Wissenschaftstheorie wurde vor allem die Frage zentral, wie sich Naturgesetze zu empirischer Allgemeinheit verhalten. Denn der Naturgesetzbegriff transportiert in seiner Herkunft aus der geometrischen Beweis- 
lehre das Moment der Notwendigkeit mit sich. Naturgesetze in diesem Sinne bezeichnen keine bloßen Regelmäßigkeiten der Natur, sondern drücken einen Moment des Zwangs aus: eine Bewegung vollzieht sich in einer bestimmten Form, weil sie sich, gemäß des Naturgesetzes, nicht anders verhalten kann (vgl. Hüttemann 149). Eine empirische Allgemeinheit wie die Aussage, dass salle Schwäne weiß sind (Chalmers, s. BASISWISSEN 6), kann nicht als Naturgesetz gelten, da jede weitere Beobachtung tatsächlich ein anderes Resultat liefern könnte. Andererseits kann der naturgesetzliche Zwang aber auch nicht mit dem Zwang logischer Schlussfolgerung identifiziert werden. Denn die natürlichen Erscheinungen verhalten sich nur unter ganz bestimmten Bedingungen so, wie die naturgesetzliche Formulierung es vorsieht. Galileis Fallgesetz gilt z.B. nur im Vakuum. Man spricht daher von Naturgesetzen als Ceteris-Paribus-Gesetzen (von lat. »anderes gleichbleibend«) und so liegt der Schluss nahe: »Naturgesetze sind typischerweise Ceteris-Paribus-Gesetze. Sie beschreiben das Verhalten von Gegenständen unter spezifischen Umständen.« (Hüttemann 152). Der Verweis auf spezifische Umstände zeigt an, wie sehr das Konzept von Naturgesetzen an die Realisierung von idealen Bedingungen gebunden ist, die in der Natur kaum je und annäherungsweise nur im Labor gegeben sind.

Michael Hampe: Eine kleine Geschichte des Naturgesetzbegriffs, Frankfurt a.M. 2007.

Gerhard Vollmer: Was sind und warum gelten Naturgesetze? In: Philosophia naturalis. Journal for the Philosophy of Nature. Band 37/2 (2000). Andreas Hüttemann : >Naturgesetze . In: Andreas Bartels/Manfred Stöckler(Hrsg.): Wissenschaftstheorie, Paderborn 2007, S. 135-154. 


\section{Wo liegt das Problem?}

Je umfassender die Wissenschaftstheorie in den Blick nahm, wie mathematisierte und experimentierende Wissenschaft tatsächlich arbeitet, desto mehr zerfiel ihr das beweistheoretische Bild und desto stärker veränderte sich ihr der Begriff des Naturgesetzes. Schon um die Jahrhundertwende um 1900 geriet das Bild von Gesetzen, die auf empirischem Wege erschlossen werden können, ins Wanken. Neben Ernst Mach und Henri Poincaré war es vor allem Pierre Duhem, der argumentierte, dass die Physik keineswegs induktiv von der Erfahrung zur Theorie voranschreitet. Am Beispiel von Ampères Theorie der Elektrodynamik zeigt Duhem, dass die experimentelle >Basis`für theoretische Schlussfolgerungen zumeist rudimentär ist, was impliziere, dass die stheoretische Fassung der Phänomene nicht empirisch vorbestimmt sein kann. In diesem Zusammenhang schreibt Duhem nun von der »Notwendigkeit für den Physiker, die experimentellen Tatsachen symbolisch auszudrücken ${ }^{51}$ und erläutert: "Die Tatsachen des Experiments wären, roh, wie sie von Natur aus sind, nicht der mathematischen Behandlung zugänglich gewesen. Um diese Behandlung zu ermöglichen müssen sie umgebildet und in symbolische Form gebracht werden. $\aleph^{52}$ Die unzureichende experimentelle Basis bedeutet nun aber für die Form der Symbolisierung, dass sie unabhängig vom Empirischen festgelegt werden muss: "Bei so wenig genauen Experimenten bleibt dem Physiker die Sorge überlassen, unter einer Unzahl gleich guter symbolischer Übersetzungen zu wählen. ${ }^{53}$ Eine solche Übersetzung ist für Duhem keine Ausnahme, sondern die Regel, sie lässt sich etwa auch in der Übertragung der Keplerschen Gesetze in die »symbolische Form « ${ }^{54}$ der Newtonschen Dynamik nachweisen. ${ }^{55}$ Diese Denkfigur einer Übersetzung aus dem

51 Pierre Duhem (im Orig. 1906): Ziel und Struktur der physikalischen Theorien, Hamburg 1998 , S. $262 f$.

52 Duhem, 1998, S. $261 f$.

53 Duhem, 1998, S. 264.

54 Duhem,1998, S. 259.

55 Instruktive Studien, wie die Multiplizierung von Möglichkeiten durch operative Symbolismen im Forschungsprozess wirksam wird, finden sich auch in den Arbeiten von Ursula Klein. Ihre Untersuchungen zum Einsatz der Formelschreibweise in der Chemie zeigen beispielsweise, wie durch Transformation der Summenformel sich immer neue Konfigurationen bilden ließen, die jeweils unterschiedliche Interpretationen über die enthaltenen Stoffe implizierten und wie dadurch eine Fülle von Ansätzen für weitere experimen- 
Empirischen ins Symbolische anstelle einer Beweisführung ist im 20. Jahrhundert von vielen Wissenschaftstheoretikern ausbuchstabiert worden.

\section{$\checkmark$ VERTIEFEND Symbolismen in den Wissenschaften}

Unter anderen haben Elisabeth Anscombe, Georg Henrik v. Wright und Thomas S. Kuhn ${ }^{56}$ Beispiele und subtile Argumente beigetragen, in jüngerer Zeit hat die Physikerin und Philosophin Nancy Cartwright die Problematik in einige prägnante Formulierungen gefasst.

Cartwrights How the Laws of Physics Lie von 1983 beschreibt das Verhältnis von Theorie, Empirie und Wirklichkeit in einem dreistufigen Schema: »The route from theory to reality is from theory to model, and then from the model to the phenomenological law. The phenomenological laws are indeed true of the objects in reality - or might be; but the fundamental laws are true only of

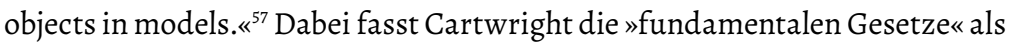
symbolische Form einer mathematischen Theorie. Der Transfer in den sym-

telle Untersuchungen entstanden. Ursula Klein (2003): Experiments, Models, Papertools. Cultures of Organic Chemistry in the Nineteenth Century, Stanford.

56 Vgl. Gertrud E. M. Anscombe: Causality and Determination. In: Metaphysics and the Philosophy of Mind (The Collected Philosophical Papers: 2), Oxford 1981; Georg H. Wright: >Laws of Nature In: Truth, Knowledge and Modality, Philosophical Papers. 3 (1984), S. 135-149; Thomas S. Kuhn: Die Entstehung des Neuen: StudienzurStrukturderWissenschaftsgeschichte, Frankfurta.M.1984. Eine besonders instruktive Darstellung findet sich in Holm Tetens Buch Experimentelle Erfahrung (Hamburg1987):DasTheoremvon Lakatos, dasswissenschaftliche»Forschungsprogramme» einen »harten Kern« enthalten, zurückverfolgend, führt Tetens den Begriff der Exhaustion von Hugo Dingler ein. Unter Exhaustion versteht Dingler die Einführung gewisser »fundamentale(r) Begriffe«»in die Wirklichkeit« und zwar »geistig, indem die Begriffe und Vorgänge, soweit sie einschlägig, in Ansatz gebracht werden und alles, was durch sie noch nicht gedeckt ist, als überlagernde Erscheinung definiert wird. Manuell geschieht sie, indem die Umstände so lange variiert werden, bis die genaue Wirkung eintritt. Dadurch erscheint dieses `Variieren der Umstände`als ein `Fortschaffen störender Umständeく. (Dingler, zitiert nach Tetens S. 8.) Tetens führt diese Überlegungen zum Justieren des Experimentalsystems folgendermaßen weiter aus: »Widersprechen experimentelle Befunde zunächst einem Fundamentalgesetz, so versuchen die Experimentatoren die [...] unterstellten störenden Faktoren durch Variieren bis dahin noch nicht berücksichtigter Umstände zu identifizieren und durch ihre Isolation oder ihr Konstanthalten, also durch technischen Umbau der apparativen Versuchsanordnung unwirksam zu machen, so daß der im Experiment schließlich herbeigeführte Verlauf immer vollkommener, störungsfreier nach den Fundamentalgesetzen des Forschungsprogramms abläuft.«Tetens, 1987, S. 9.

57 Cartwright, Nancy: How the Laws of Physics Lie, New York 1983, S. 4. 
bolischen Formalismus ist für Cartwright nicht einfach eine Repräsentation, vielmehr eine Übersetzung in ein anderes mediales System eigener Gesetzmäßigkeit. Cartwright beschreibt dies (nun in umgekehrter Richtung) folgendermaßen: »I think theory entry proceeds in two stages. We start with an unprepared description, which gives as accurate a report as possible of the situation. The first stage converts this into a prepared description. At the second stage the prepared description is matched to a mathematical representation from the theory. Ideally the prepared description should be true to the unprepared. But the two activities pull in opposite directions, and a description that is adequate to the facts will seldom have the right mathematical structure. ${ }^{58}$

Betrachtet man den Symbolismus als eigenständige mathematische Struktur, als Darstellungs- und Folgerungstechnik, so ist klar, dass er nicht schlechthin die Ordnung der Wirklichkeit zu repräsentieren vermag, dass er vielmehr ein Ordnungssystem darstellt, das eigenen Regeln und Strukturbedingungen gehorcht.

Die Rolle von "präparierten Beschreibungen« oder »Modellen« als Mediatoren zwischen Empirie und Theorie, wie Cartwright sie beschreibt, wurde in der Folgezeit zu einem viel diskutierten Thema. ${ }^{59}$ Dass diese Rolle eine doppelte ist, hatte bereits Thomas S. Kuhn in seinem Postscriptum (von 1969) zu seiner Theory of Scientific Revolutions gezeigt: Kuhn legt auseinander, dass "symbolische Verallgemeinerungen «, also jene »Formeln, die problemlos von allen Gruppenmitgliedern gebraucht werden und ohne weiteres z.B. auf die logische Form (x) (y) (z) $\phi(x, y, z)$ gebracht werden können « ${ }^{60}$ für die Wissenschaftler sowohl die Rolle von Naturgesetzen als auch definitorische Funktion haben. Das heißt, dass sie sowohl die Rolle von Instrumenten als auch die Rolle von Resultaten spielen können - und zwar so, dass sie zwischen diesen beiden Rollen auch wechseln können, je nachdem, wie das »disziplinäre System « einer Wissenschaft ausgeprägt ist. ${ }^{61}$

58 Cartwright, 1983, S. 15.

59 Ulrich Gähde: Modelle der Struktur und Dynamik wissenschaftlicher Theorien. In: Andreas Bartels/Manfred Stöckler (Hrsg.): Wissenschaftstheorie, Paderborn 2007, S. 45-68, hier S. 64. Siehe dort zu weiterer Literatur zum Thema.

60 Thomas S. Kuhn: Die Entstehung des Neuen: Studien zur Struktur der Wissenschaftgeschichte, Frankfurt a.M.; S. 194.

61 Kuhn, 1988b, S. 198. 
Wir können für den Typus Laborwissenschaften also festhalten:

Theoretische Modelle sind in den Laborwissenschaften keine Repräsentationen der Wirklichkeit, sondern Übersetzungen in ein System, das seine eigene Logik hat. Soweit dies nur die Einsicht in die Unabhängigkeit zumindest einiger Teile einer jeweiligen Theorie von empirischen Befunden betrifft, ist dieser Punkt für die Wissenschaftsphilosophie seit Duhem trivial geworden. Doch die Einsicht, dass diese Unabhängigkeit besteht wie sie besteht, weil die Übersetzung eine Übersetzung in ein Symbolsystem mit symbolsystemischen Implikationen ist, ist alles andere als trivial. Denn zu diesen symbolsystemischen Implikationen gehört, dass sie als Folgerungstechniken zu gebrauchen sind, das heißt: Was herkömmlicherweise Theorie heißt, stellt sich hier als ein eigenständiges Feld der Praxis dar. Wir haben es mit zwei Feldern von Praxis zu tun, die auf eine für den Typus Laborwissenschaften charakteristische Weise miteinander korreliert sind.

\section{Noch einmal zum Verstehen: Das Forschungsparadigma der Hermeneutik - Was haben Verstehenslehren mit Kriminalistik zu tun? Der Ansatz der Rekonstruktion}

Ebenso wie die Scientia-Tradition bildet auch die Verstehenslehre einen Komplex von Ideen und Verfahren, der nur aus der Ferne wie eine geschlossene Einheit aussieht. Schauen wir näher hin, dann erkennen wir auch hier mindestens drei verschiedene Ansätze dafür, was Wissenschaft im Kern ausmacht.

Einer dieser Ansätze ist mit dem Begriff der Rekonstruktion, mit der Beziehung von Teil und Ganzem - und damit auch mit Analyse und Synthese verbunden. Um welche Disziplinen geht es, und um welche Zeit?

Herausgebildet hat sich dieser Ansatz in der ersten Hälfte des 19. Jahrhunderts, und zwar in den - zu dieser Zeit erstarkenden - Philologien und Geschichtswissenschaften, mit Ausstrahlung in die Rechtswissenschaften, aber auch in Naturphilosophie und Erkenntnistheorie. Seine populäre und wirkmächtige Form fand er schließlich gegen Ende des 19. Jahrhunderts in Wilhelm Diltheys Abgrenzung einer Methodik der Geisteswissenschaften von einer der Naturwissenschaften. Doch hatte sich hier längst das Paradigma der hermeneutischen Forschung mit einer ganzen Reihe anderer Motive vermengt, sodass seine Konturen nur mehr schwer zu erkennen sind. Sehen 
wir genau hin und lassen uns nicht irritieren von einer Sprache, die uns heute unvertraut ist.

Viele der uns in diesem Feld begegnenden Vokabeln - wie Intuition, Divination, Empathie, Mitwisserschaft, Mitgefühl ${ }^{62}$ - erwecken den Anschein, dass es sich beim Verstehen um eine irrationale Sache des Einfühlens handele. Doch dem Forschungsparadigma der Hermeneutik liegen sehr klare, strukturelle Überlegungen zugrunde. So bedeutet z.B. Divination, ein Begriff, den Friedrich Schleiermacher, der gemeinhin als Begründer einer wissenschaftlichen Hermeneutik gilt, ${ }^{63}$ prominent machte, schlicht vermuten und erraten (von lateinisch: divinare). Und dass ein solches Moment nötig ist, liegt schon an der Aufgabenstellung: Texte bestehen aus Teilen, Wörtern, Sätzen, Abschnitten. Die Bedeutung eines Textes, also wie er zu verstehen ist, hängt von seinen Teilen ab. Umgekehrt hängt aber die Bedeutung eines jeden Teils von seinem jeweiligen Kontext, letztlich vom Ganzen ab. Teil und Ganzes bestimmen sich gegenseitig. Das heißt, dass jeder Teil mit Blick auf seine möglichen Rollen im Ganzen und die Auffassung des Ganzen aus möglichen Arrangements der Teile entwickelt wird. Weder Teile noch Ganzes sind fix vorgegeben, sodass in die eine oder andere Richtung schlicht abgeleitet werden könnte.

Die für das Textverstehen charakteristische Bewegung zwischen Teil und Ganzem hat auch in den Geschichtswissenschaften einen zentralen Stellenwert. Denn auch hier gibt es, so Johann Gustav Droysen, wichtigster Methodologe der Historik im 19. Jahrhundert, keinen fixen Ausgangspunkt, von dem her sich ein historisches Phänomen erklären ließe. Droysen lehnt jedes Denken $a b$, das einen absoluten Anfang, einen Ursprung oder ein Wesen einer historischen Erscheinung annimmt. »[E]s ist einfach eine methodische Gedankenlosigkeit «, schreibt er, »wenn man forschend zu einem Punkt gelangen zu können meint, der anders als relativ der Anfang wäre, d.h. der sich anders als in dem, was daraus geworden ist, als Anfang ausweist $« .{ }^{64}$ Das

62 Meinrad Böhl/Wolfgang Reinhard/Peter Walter (Hrsg.): Hermeneutik. Die Geschichte der abendländischen Textauslegung von der Antike bis zur Gegenwart, Wien u.a. 2013, S. 442-548, hier S. 385.

63 Vgl. dazu den Eintrag >Verstehen<, in: Joachim Ritter (Hrsg.): Historisches Wörterbuch der Philosophie, Bd. 11, Basel 2001, S. 47291-47350, hier S. 47298-47302.

64 Johann Gustav Droysen: Historik. Band 1. Historisch-kritische Ausgabe von Peter Leyh, Stuttgart 1977 [1857/1858], S. 160. 
bedeutet, »dass wir [...] das Nacheinander, zu dem wir uns forschend das Gewordene rekonstruieren, nur nachzuahmen versuchen «. ${ }^{65}$

Dieser Begriff des Rekonstruierens ist zentral für das Forschungsparadigma der Hermeneutik. Im Wort Rekonstruktion steckt schon, dass der Erkenntnisgegenstand zusammengefügt (wörtlich: zusammengeschichtet) ist. Das Bedürfnis zu rekonstruieren kann nun aus zwei Gründen entstehen. In dem einen Fall sind von einem ehemaligen Zusammenhang nur Reste, Fragmente oder indirekte Zeugnisse wie Indizien, Symptome oder Spuren gegeben. Rekonstruieren bedeutet dann - wie bei einem Puzzle - die ursprüngliche Zusammensetzung so weit wie möglich wieder herzustellen.

Im anderen Fall bedeutet Rekonstruieren, zu einer gegebenen komplexen Erscheinung die Bedingungen ihrer Entstehung zu rekonstruieren, also: diese Bedingungen so weit aufzuklären, dass das Zustandekommen der Erscheinung verständlich, als schlüssige Zusammenführung der sie konstituierenden Faktoren erscheint. Der erste Fall kann als synthetische, der zweite als analytische Rekonstruktion bezeichnet werden. Doch in welchem Sinne Synthese und in welchem Analyse? Droysen hat, den zweiten Fall im Blick, eine prägnante Formulierung gefunden, was analytisches Verstehen in Abgrenzung vom szientifischen Paradigma bedeutet:

»Wir erklären nicht. Interpretation ist nicht Erklärung des Späteren aus dem Früheren, des Cewordenen als ein [notwendiges] Resultat der historischen Bedingungen, sondern ist Deutung dessen, was vorliegt, gleichsam ein Lockermachen und Auseinanderlegen dieses unscheinbaren Materials nach der ganzen Fülle seiner Momente, der zahllosen Fäden, die sich zu einem Knoten verschürzt haben, das durch die Kunst der Interpretation gleichsam wieder rege wird und Sprache gewinnt. ${ }^{66}$

Analyse (von analysis: Auflösung) bedeutet hier also nicht eine Aufteilung in einzelne, konstitutive Elemente, sondern ein Sichtbarmachen der Verhältnisse zwischen den Teilen, deren Beziehung dabei erhalten bleibt. Es geht um das Aus-Legen des Materials, um seine Ent-Faltung, um explicare (ausfalten), nicht um explanare (ausebnen).

65 Droysen, 1977, S. 159.

66 Droysen, 1977, S. 163. 


\section{BASISWISSEN 8: Analyse und Synthese - ein Begriffspaar fährt Achterbahn}

Das Begriffspaar Analyse und Synthese gehört zu den kompliziertesten Konzepten der Philosophie- und Wissenschaftsgeschichte. Dabei sind nicht die Worte das Problem: die griechische Vokabel analysis bedeutet Auflösung, synthesis Zusammensetzung. Kompliziert wird die Sache dadurch, dass sich in ihr Grundausrichtungen der Erkenntnistheorie niederschlagen - die Gegensätze von aristotelischer, euklidischer und atomistischer Auffassung von Erkenntnis prägen das Paar.

Zur Begriffsgeschichte: Das Begriffspaar Analyse/Synthese stammt, anders als häufig angenommen, nicht aus der antiken Philosophie. Nur analysis kann als ein Schlüsselbegriff bei Aristoteles gelten - titelgebend für zwei seiner Hauptwerke (Erste Analytik und Zweite Analytik). Doch es findet sich in den aristotelischen Werken keine Textstelle, in der analysis und synthesis auch nur gemeinsam erwähnt werden. Liest man zuweilen, Aristoteles habe methodische Leitlinien aus den Euklidischen Elementen übernommen, so steht dem schon die Chronologie entgegen.

Die Zusammenstellung von Analyse und Synthese taucht vermutlich erstmals in einem Zusatz zu Die Elemente von Euklid auf, der von dem um $100 \mathrm{n}$. Chr. lebenden Mathematiker und Ingenieur Heron von Alexandrien stammt. Dort liest man:

»Was ist eine Analysis und was eine Synthesis? Eine Analysis ist die Zugrundelegung des Gefragten als anerkannt um seiner auf anerkannt Wahres führenden Folgerungen willen. Eine Synthesis ist die Zugrundelegung des Anerkannten um seiner auf Vollendung oder Ergreifung des Gefragten führenden Folgerungen willen.«

Unschwer ist zu erkennen, dass der Kontext hier die geometrische Beweislehre ist, die als Erkenntnis more geometrico in der frühen Neuzeit Furore macht und sich von der aristotelischen Beweislehre genau in dem Punkt unterscheidet, dass Folgerungen bei Aristoteles keine Konstruktionsschritte sind, die das Gefolgerte tatsächlich produzieren (siehe oben 
BASISWISSEN 5). Deshalb ist der Komplementärbegriff zu Analysis bei Aristoteles nicht synthesis, sondern syllogismos, das heißt, die Zusammenstellung von Aussagen zu logischen Schlussfolgerungen. Synthesis wiederum verwendet Aristoteles in Kontexten der konkreten Zusammensetzung von Elementen, z.B. von Buchstaben zu Silben oder von Bausteinen zu einem Haus. Diese Verwendungsweise erklärt auch, warum in der aristotelischen Erkenntnislehre Synthese nicht das Gegenbild zu Analyse sein kann: An Elementen wie Buchstaben oder Bausteinen lässt sich nicht ablesen, wie sie zu sprachlichen Einheiten oder Gebäuden gefügt werden können, wer nicht über die Prinzipien der Sprache oder der Architektur verfügt. Diese Prinzipien sind deshalb das Ziel der aristotelischen analysis - nicht die synthetisierbaren Elemente (was einer atomistischen Erkenntnistheorie entspräche).

Die Idee einer Synthesis aus Prinzipien konnte nur über den Sonderfall des Mathematischen, in dem eine Schlussfolgerung zugleich eine Realkonstruktion aus Elementen, nämlich mathematischen Formen oder Buchstaben, bedeutet, in die Neuzeit vermittelt werden. Verbunden mit der christlichen Welt eines Schöpfergottes und mit dem neuen Selbstverständnis des Menschen als Homo faber konnte nun Wissenschaft als Disziplin der technischen Herstellung auf Grundlage mathematischer Konstruktion verstanden werden. Und dennoch: Der Glaube an die synthetische Herstellbarkeit beliebiger Produkte ist und bleibt ein Glaube, der die Geister scheidet. (Kogge/ Richter 2013)

Gabriele Gramelsberger/Peter Bexte/Werner Kogge (Hrsg.), Synthesis. Zur Konjunktur eines philosophischen Begriffs in Wissenschaft und Technik, Bielefeld 2013.

Werner Kogge/Michael Richter: »Synthetic biology and its alternatives:

Descartes, Kant and the Idea of Engineering Biological Machines«. In: Studies in History and Philosophy of Biological and Biomedical Sciences. Special Issue: >Philosophical Perspectives on Synthetic Biology<, Vol. 44, Issue 2, June 2013, S. 181-190. 
In der von Droysen pointierten Bewegung einer Analyse als ein Auseinanderziehen zeigt sich auch deutlich der Unterschied zur Auffassung von Wissenschaft als Beweisen und Erklären: Prinzipien und Axiome stehen dort als Ausgangspunkte fest, während im Verstehen die organisierenden >Prinzipien $<$ des Textes allererst erschlossen werden. Verstehen charakterisiert sich deshalb als eine suchende Bewegung des probeweisen Vorwegnehmens in einem Prozess über mehrere Stufen. Typischerweise läuft ein solcher Prozess etwa so ab: Wenn wir a als $\mathrm{x}$ auffassen, dann stellt sich der Zusammenhang so ... dar, was wieder bedeuten würde, dass b als y aufzufassen wäre und c als z, was aber nicht plausibel ist, weil ... eher könnte ... usw. Die Struktur kennen wir vom Rätsellösen und von vielen anderen Konstellationen, in denen es um Rekonstruktion von Zusammenhängen geht, etwa in der Aufklärung von Kriminalfällen und in der historischen Forschung.

Wie ein roter Faden zieht sich das so beschriebene Grundmotiv durch die verzweigte Geschichte der Verstehenslehren. Dass es auf den jeweiligen Kontext ankommt, erklärt schon Augustinus, ${ }^{67}$ und die Rolle der ScientiaDisziplinen besteht für ihn in nichts anderem als darin, Kontextwissen zum Verständnis des rätselvollen Zusammenhangs der Heiligen Schrift bereitzustellen. Zwar geht von Augustinus auch die Vorstellung aus, unklare Textstellen durch Vergleich mit klaren zu verstehen; und damit auch die Vorstellung von einer Kunst der Auslegung, die nach Regeln verfährt. Doch sind auch die Kunstlehren des Verstehens, die explizit auf rationale Verfahren setzen, dem forscherischen Motiv des Verstehens verpflichtet. Friedrich Schleiermacher drückt die Konstellation des Verstehens in der Formulierung aus, dass wir »öfter vom Ende zum Anfang zurückkehren und das Auffassen ergänzend von neuem beginnen; je schwieriger die Gliederung des Ganzen zu fassen ist, desto mehr suchend ihr vom Einzelnen aus auf die Spur [...] kommen, je reichhaltiger und bedeutsamer das Einzelne ist, um so mehr suchend es vermittelst des Ganzen in all seinen Beziehungen [auffassen]. ${ }^{68}$

Überhaupt spielt in diesem Forschungsparadigma das Detail in seiner Aussagekraft für das Ganze eine entscheidende Rolle: feine Unterschiede

67 Aurelius Augustinus: Die christliche Bildung (De doctrina christiana) [426/427], Stuttgart 2002, Zweites Buch, XII, 42; Drittes Buch XXV, 37.82 (S. 60 u. S. 128).

68 Friedrich Schleiermacher: Über den Begriff der Hermeneutik mit Bezug auf F.A. Wolfs Andeutungen und Asts Lehrbuch. In: ders.: Hermeneutik und Kritik, Frankfurt a.M. 1977, S. 309-346, hier S. 333f. 
sind hier wichtig. Carlo Ginzburg, italienischer Historiker und Kulturwissenschaftler, publizierte im Jahr 1979 Spie. Radici di un paradigma indiziaro. Ginzburg entwickelt hier den Begriff eines Indizienparadigmas als einen neuen Typ von Wissenschaft - dessen Verwandtschaft mit dem Forschungsparadigma der Hermeneutik aber auf der Hand liegt: wenn der Kunsthistoriker Giovanni Morelli Gemälde an wenig beachteten Details identifiziert, wenn Sherlock Holmes einen Tathergang aus der Beschaffenheit von Zigarettenasche erschließt und Freud psychische Konstellationen vom Lapsus her interpretiert, dann werden anhand von Details, Relikten und Unwillkürlichkeiten Rückschlüsse auf Gesamtzusammenhänge gezogen. Auch Ginzburg spricht davon, dass es in diesem Typ von Wissen darum geht, mithilfe »kleinster Indizien [etwas zu] rekonstruieren « ${ }^{69}$

Die Arbeiten von Gustav Droysen und Carlo Ginzburg trennen gut hundert Jahre. Und auch ansonsten scheint sie nicht viel zu verbinden. Doch betrachtet man beide in Hinblick auf das für die Geschichtswissenschaften zentrale Verfahren der Rekonstruktion, dann zeigen sie sich gemeinsam als Vertreter eines wissenschaftlichen Paradigmas - nämlich des Forschungsparadigmas der Hermeneutik. Ginzburg hebt sein Indizienparadigma vom Galileischen Paradigma der Wissenschaft ab und schreibt:

»An diesem Punkt eröffnen sich zwei Möglichkeiten: entweder man opfert die Erkenntnis des individuellen Elementes zugunsten der (mehr oder weniger streng mathematisch formulierbaren) Verallgemeinerung, oder man versucht - sich langsam vortastend - ein anderes Paradigma zu erarbeiten, das sich auf die wissenschaftliche Erkenntnis des Individuellen stützt (wobei es sich um

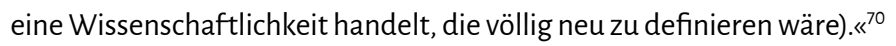

Dass diese Form der Wissenschaftlichkeit weder neu noch erst zu definieren wäre, haben wir gesehen. Aber halten wir fest: In einer Hauptlinie lässt sich die hermeneutische Tradition als Kern von Forschung im buchstäblichen Sinne auffassen. Re-Search/Forschung: wo geforscht wird, wird gesucht,

69 Carlo Ginzburg: Spurensicherung. Der ]äger entziffert die Fährte, Sherlock Holmes nimmt die Lupe, Freud liest Morelli - Die Wissenschaft auf der Suche nach sich selbst. In: ders.: Spurensicherung. Über verborgene Geschichte, Kunst und soziales Gedächtnis, München 1988, S. 78-125, hier S. 88 [Herv. WK] (im Orig.: Spie. Radici di un paradigma indiziaro, Torino 1979). 70 Ginzburg, 1988, S. 108. 
probiert, variiert, angepasst, neu konzipiert - unabhängig davon, ob die Teil-Ganzes-Relation zwischen Textstelle und Textganzem, zwischen historischem Akteur und politischem Geschehen oder zwischen Mikroorganismus und Krankheit methodisch hin- und hergeht. Das Kreuzworträtsel und das Puzzle sind Modelle einer solchen Konzeption. Und daher ist es ist kein Zufall, dass Thomas S. Kuhn das Puzzle-Solving als die Praxis wissenschaftlichen Forschens schlechthin angesehen hat. ${ }^{71}$ In jedem Fall geht es - wie in einem Kriminalfall - darum, Rätselhaftes zu entschlüsseln, indem es einem Gesamtzusammenhang schlüssig integriert wird, der selbst erst zu rekonstruieren ist. Das hermeneutische Verfahren stellt sich im Paradigma der Rekonstruktion - analytisch oder synthetisch - als ein Hin- und Hergehen in der Teil-Ganzes-Relation dar. Dabei ist keiner der beiden Pole der Bewegung fix gegeben, vielmehr erschließt sich der Sinn des Ganzen sukzessive aus den Details und die Rolle der Details aus ihrer Position im Zusammenhang.

\section{Adäquatheit - Von der Anpassung der Konzepte zur Einfühlung und zurück}

In der Tradition von Interpretation und Hermeneutik verschachteln und verzweigen sich aber auch andere Motive. So ist es eine der ältesten und uns auch geläufigsten Vorstellungen, dass Erkenntnis darin besteht, das Auffassen in Übereinstimmung mit dem Gegenstand zu bringen. Ob wir Konzept, Begriff, Repräsentation, Beschreibung oder - traditionellerweise Idee oder Intellekt dazu sagen: Immer geht es darum, dass Form und Struktur, worin wir etwas auffassen, dem entspricht oder sich zumindest dem angleicht, was aufgefasst wird.

71 »Die Metapher des >Rätsel-Lösen [puzzle-solving]< zur Charakterisierung der normalen Wissenschaft spielt in Kuhns Schriften an vielen Orten eine zentrale Rolle. Kuhn hat als Quelle der Analogie etwa Kreuzworträtsel, Zusammensetzspiele (Puzzles) und Schachprobleme im Blick. Für Kuhn ist die Analogie der normalwissenschaftlichen Forschungsarbeit mit dem Rätsellösen so tiefgehend, dass er am ehesten in dieser Analogie ein Abgrenzungskriterium sieht, mit dem die Wissenschaften von anderen kreativen Unternehmen - wie den Künsten in einem weiten Sinn oder der Philosophie unterschieden werden können.«Paul Hoyningen-Huene: Die Wissenschaftsphilosophie des Thomas S. Kuhns. Rekonstruktion und Crundlagenprobleme, Braunschweig 1989, S. 168, mit einer Fülle von Belegen. 
Die Idee des Verstehens durch Angleichung lässt sich zurückverfolgen bis zu Textstellen bei Platon, sie findet eine Widerspiegelung in der Schrift perí hermēneías des Aristoteles (Verstehen als sympatheia: „Verstehen [...] findet dann statt, wenn der verstehenden Seele dasselbe widerfährt, was der Seele des Sprechers widerfuhr ${ }^{7^{2}}$ ) sie lässt als homoion-homoio-Prinzip (»Gleiches zu Gleichem «) an diversen Stellen im antiken Denken nachweisen, ${ }^{73}$ taucht beim Neuplatoniker und Aristoteles-Kommentator Porphyrios in dem Satz, "alle Erkenntnis sei eine Verähnlichung mit dem Erkannten « ${ }^{74}$ auf und wird im Mittelalter zur stehenden Wendung: »jede Erkenntnis erfolgt durch eine Art Angleichung [assimilatio] des Erkennenden an das Erkannte ${ }^{75}$, schreibt Thomas von Aquin und Nikolaus von Kues spricht von einer »vis assimilativa« als einer »Fähigkeit, sich selbst den Gegenständen zu verähnlichen (sse assimilare $) \ll \cdot{ }^{76}$ Die »adaequatio rei et intellectus«, die Thomas von Aquin als eine klassische Wahrheitskonzeption referiert, gehört ebenfalls hierher. ${ }^{77}$

Dieser Gedanke einer Angleichung oder Anpassung des Intellekts an die Sache blieb aber stets im Kontext der Angleichung an die Struktur einer als göttlich oder kosmologisch gedachten Weltordnung. Was nun im 19. Jahrhundert neu ist, sich zumindest hier erst deutlich ausprägt, ist die Übertragung dieses Motivs auf das Verstehen individueller Autoren oder historischer Akteure.

Schleiermacher hatte als Aufgabe bestimmt, »den ganzen inneren Verlauf der komponierenden Tätigkeit des Schriftstellers auf das vollkommenste nachzubilden ${ }^{78}{ }^{78}$ Dies ließ sich unter dem Aspekt umsetzen, dass man versteht, indem man den Entstehungsprozess von den Anfängen zum Ganzen rekonstruiert, aber auch so, dass man versteht, indem man sich dem Gegenstand anverwandelt. Zwei Relationen: zum einen die von Teil und

72 Matthias Jung/Arne Moritz/Magnus Schlette: Philosophie. In: Meinrad Böhl/Wolfgang Reinhard/Peter Walter Hermeneutik. Die Geschichte der abendländischen Textauslegung von der Antike bis zur Gegenwart, Wien u.a. 2013, S. 442-548, hier S. 447f.

73 Axel Horstmann: Das Fremde und das Eigene - >Assimilation< als hermeneutischer Begriff. In: Archiv für Begriffsgeschichte, Bd. 30, Bonn 1986/87, S. 7-43.

74 Vgl. Horstmann, 1986-87, S. 15.

75 Vgl. Horstmann, 1986-87, S. 15.

76 Vgl. Horstmann, 1986-87, S. 18.

77 Vgl. den Eintrag >Wahrheit< in: Joachim Ritter, Joachim (Hrsg.): Historisches Wörterbuch der Philosophie, Bd. 12, Basel 2005, S. 48552-48784, hier S. 48612.

78 Schleiermacher 1977, S. 321. 
Ganzem, zum anderen die von Ich und $\mathrm{Du}$. Die zweite bestimmte die romantische Hermeneutik und das Bild, das in der Folgezeit die Vorstellung vom Verstehen als Einfühlen beherrscht. So formulierte etwa Droysen (allerdings nur in Bezug auf einen Typ Interpretation - die spsychologische`), dass wir um »zu verstehen gleichsam in ihre Seele [der schöpferisch Tätigen, Anmerkung des Verfassers] hineinzuversetzen suchen müssen, um den Tatbestand [...] auch in seinem Werden durch den Willen und die Leidenschaften der Führenden und Handlenden zu erkennen. $\ll^{79}$ Radikaleres lesen wir aus dieser Zeit bei dem Historiker Leopold v. Ranke (»Ich wünschte mein Selbst gleichsam auszulöschen ... $\ll^{80}$ ) und bei dem Altphilologen August Boeckh (»[es] wäre aber nöthig, dass man vollständig in eine fremde Individualität einginge $\ll^{81}$ ).

Ein Feld, in dem diese Differenz von Wissenschaftskonzeptionen im 20. Jahrhundert ausgetragen wurde, ist eine wissenschaftstheoretische Kontroverse in der Ethnologie und Cultural Anthropology. Ausgangspunkt war das Bemühen, die Ethnologie stärker wissenschaftlich auszurichten, und zwar sollte dies - so Bronislaw Malinowski, der führende Akteur dieser Bewegung in den 1910 bis 1940er Jahren - durch Feldforschung einerseits und durch funktionale Analyse der Strukturen einer Gesellschaft andererseits geschehen.

Allerdings wurde dieser Ansatz seit Ende der 1960er Jahre mehr und mehr infrage gestellt: Ist es überhaupt möglich, eine angemessene Beschreibung einer fremden Kultur zu erstellen, wenn diese Beschreibung doch stets in der jeweils eigenen Kultur des Wissenschaftlers erfolgt? Projizieren wir nicht nur unsere eigenen kulturellen Denkmuster auf die Gesellschaft, die wir untersuchen?

Als Reaktion auf dieses Dilemma kristallisieren sich zwei Forschungsansätze heraus, die den beiden hermeneutischen Paradigmen der forschenden Rekonstruktion und der Adäquation entsprechen. Zum einen nämlich hat Clifford Geertz, ein US-amerikanischer Anthropologe, der in den 1960er bis 1990er Jahren die Debatte mitprägte, einen hermeneutischen Ansatz ent-

79 Droysen, 1977, S. 165.

80 Leopold von Ranke: Sämmtliche Werke, 54 Bde (Leipzig 1867-90) Bd. 15, 103. Zitiert nach Horstmann, 1986/87, S. 26.

81 August Boeckh: Encyclopädie und Methodologie der philologischen Wissenschaften, hrsg. v. Ernst Bratuscheck, Wiesbaden 1886, S. 140. 
wickelt, der das rekonstruktive Forschungsparadigma wieder aufrief: Kulturen seien »selbstgesponnene [...] Bedeutungsgewebe «, ${ }^{82}$ die sich aus dem Selbst- und Weltverständnis ihrer Mitglieder ergeben. Ethnographische Forschung bestehe daher im Kern darin, das Gewebe dieses Verständnisses zu rekonstruieren. Im Unterschied zum Forschungsansatz der Malinowski-Schule gibt es für Geertz keine universalen sozialen Strukturen, die am Gegenstand aufgedeckt werden können, sondern je spezifische - individuelle - Bedeutungswelten, die mit Sinn fürs Detail und Gespür für weite Zusammenhänge nachvollzogen, oder - wie Geertz es ausdrückt, »über die Schultern derjenigen, für die sie eigentlich gedacht sind, zu lesen « sind..$^{83}$

Vom Denken der romantischen Hermeneutik des 19. Jahrhunderts grenzt sich Geertz mit solchen Worten ab: »Es geht nicht darum, eine innere Korrespondenz mit seinen Informanten herzustellen, die daraufhin ohnehin keinen besonderen Wert legen, da sie, wie wir alle, ihre Seele lieber als ihre eigene Angelegenheit betrachten. Es geht vielmehr darum herauszufinden, wie sie sich überhaupt selbst verstehen. ${ }^{84}$

Doch auch die andere Seite entwickelte eine in sich kohärente Argumentation: Wenn nämlich klar ist, dass jede Interpretation auch eine Übersetzung in den Verständnishorizont einer wissenschaftlichen Community ist und dass damit, trotz aller Bemühung, den Gegenstand aus sich heraus zu verstehen, ein Moment der Projektion eingeht, dann bleibt jede Interpretation hinter ihrem selbstgesteckten Objektivitätsanspruch zurück. Es bleibt dann nur, wie Vincent Crapanzano schreibt, sich in einem Prozess »beständiger Reinterpreatation und Neuanpassung ${ }^{85} \mathrm{zu}$ halten. So manifestierte sich in der Ethnologie das Gebot der Anpassung der Konzepte an den Gegenstand als ein radikal selbst-kritisches und gerade deshalb - in einem bestimmten avancierten Sinne - wissenschaftliches Unternehmen.

Im Resultat führte diese Radikalisierung wissenschaftlicher Objektivität aber dazu, dass hinter der Fokussierung auf das Gepräge des Erkenntnisvor-

82 Clifford Geertz (im Orig. 1973): Dichte Beschreibung. Beiträge zum Verstehen kultureller Systeme, Frankfurt a.M. 1991, S. 9.

83 Geertz,1991, S. 259. Vgl. dazu BirgitGriesecke: Japandicht beschreiben. Produktive Fiktionalität in der ethnographischen Forschung, München 2001.

84 Geertz, 1991, S. 292.

85 Vincent Crapanzano:, Tuhami. Porträt eines Marokkaners, Stuttgart 1983, S. 184. Vgl. dazu Werner Kogge: Die Grenzen des Verstehens: Kultur-Differenz-Diskretion, Weilerswist 2002, S. 143 f. u. S. 153-162. 
gangs der Gegenstand - hier: die untersuchte Kultur - in den Hintergrund rückte. Auch das wiederum erwies sich als schwer vereinbar mit Geboten von Wissenschaftlichkeit. So zeigt sich, dass Ansprüche an Wissenschaftlichkeit ab einem bestimmten Punkt ihrer Radikalisierung mit anderen solchen Ansprüchen in Konflikt geraten können.

Das sieht nach Dilemma aus. Doch, pragmatisch gewendet, läuft das Adäquationsparadigma schlicht auf die Frage zu: Wie muss ich meine eigenen Begriffe, mein Vorwissen, die Struktur meiner Überzeugungen und Auffassungen befragen und neu formieren, um den Eigenarten meines Erkenntnisgegenstandes gerecht $\mathrm{zu}$ werden ${ }^{86}$ Und dies ist sicherlich ein Wissenschaftsideal von eigenem Wert.

\section{$\checkmark$ VERTIEFEND Zur Begriffsgeschichte von sObjektivitätı}

Die Idee, einer Sache wissenschaftlich gerecht zu werden, indem die eigenen Formen der Auffassung verändert und der Sachlage angepasst werden - diese Idee ist eng mit der Begriffsgeschichte von Objektivität verbunden. Werfen wir einen Blick auf diese Geschichte, um zu sehen, welche Verwicklungen es so schwierig machen zu klären, was es bedeutet, wenn sich Wissenschaft heute auf Objektivität beruft.

Das Wort Objektivität geht auf das mittelalterlich-lateinische Adjektiv obiective und das wiederum auf das Nomen obiectum zurück. Die spannende Wendung im Begriff Obiectum liegt darin, dass ein spätantiker Aristoteleskommentator und frühchristlicher Autor, nämlich Anicius Manlius Severinus Boethius (ca. 480-526), die griechisch-antike Erkenntnistheorie mit diesem neuen Begriff versah und ihr damit einen ganz neuen Dreh gab.

Der Grundgedanke der klassisch-antiken Erkenntnistheorie besteht darin, Erkenntnis als ein Streben (orexis) anzusehen, bei dem das Zu-Erkennen-

86 Bemerkt werden soll an dieser Stelle, dass die Hermeneutik des 19. Jahrhunderts mit ihrem lebensphilosophischen Rückgriff das Problem süberwand, indem sie es schlicht aussetzte und zwar, indem sie eine Kontinuität in der menschlichen Natur und Ceschichte voraussetzte (Dilthey: »Das Verstehen ist ein Wiederfinden des Ich im Du ... (Dilthey, Wilhelm (im Orig. 1910): Der Aufbau der geschichtlichen Welt in den Ceisteswissenschaften, Frankfurt a.M. 1993, S. 235), worauf wiederum die philosophische Hermeneutik des 20. Jahrhunderts (Heidegger, Gadamer, Ricoeur, Taylor) im Sinne vertiefter Problemwahrnehmung reagierte. Vgl. Werner Kogge: Verstehen und Fremdheit in der philosophischen Hermeneutik: Heidegger und Gadamer, Hildesheim 2001. 
de die Erkenntnisbemühung in Bewegung setzt und auf sie anziehend wirkt, sodass das Erkennen wiederum sich auf das Zu-Erkennende als Zielpunkt ausrichtet. Wir haben es also mit einer Denkfigur der Entelechie (wörtlich: 'Ins-Ziel-Haltens) und der Angleichung des Erkennens an das Erkennbare zu tun. Platons Sonnengleichnis in der Politeia (VI. Buch) und Aristoteles Ausführungen Über die Seele (Buch II u. III) liefern ausführliche Darstellungen dieser Denkfigur.

Für das Strebensziel des Erkennens gibt es im griechischen Denken aber kein Wort wie Gegenstand oder Objekt. Die Texte sprechen stets vom Wahrnehmbaren und Erkennbaren (erst die Übersetzungen ergänzen hier regelmäßig Objekt). Ein Begriff für ein solches Gegenüber (wörtlich: Entgegengeworfenes; Entgegenstehendes) hat erst viele Jahrhunderte später eben jener Boethius geprägt, indem er das in vielen Kontexten geläufige lateinische Wort obiectum in diesen Kontext einführt:

„Wenn aber bei der Wahrnehmung der Körper (in corporibus sentiendis), obwohl hierbei von aussen (forinsecus) entgegentretende Beschaffenheiten (obiectae qualitates) die Sinneswerkzeuge (instrumenta sensuum) beeinflussen (afficiant) und das leidende Empfinden (passio) des Körpers der Kraft des tätigen Ceistes vorausgeht, was die Tätigkeit des Geistes auf sich lenkt (provocet) und die inzwischen innen (intrinsecus) ruhenden Formen erweckt (excitet), wenn [...] bei der Wahrnehmung der Körper der Geist nicht durch Leiden Eindrücke erfährt, sondern aus eigener Kraft die vom Körper abhängige Empfindung (subiectam corpori passionem) beurteilt, um wieviel mehr folgt das, was von allen Berührungen durch Körper frei ist, beim unterscheidenden Erkennen nicht dem von aussen Entgegentretenden (obiecta extrinsecus), sondern setzt vielmehr die Tätigkeit des eigenen Ceistes in Bewegung. (87 $^{87}$

87 Anicius Manlius Severinus Boethius: Consolatio philosophiae III, 7. Prosa-10. Prosa. In: Trost der Philosophie. Übers. u. hrsg. v. Karl Büchner. Mit einer Einf. v. Friedrich Klingner, Stuttgart 1971. Zitiert nach: Kurt Flasch: Ceschichte der Philosophie in Text und Darstellung. Mittelalter, Stuttgart 1982, S. 125. 
Indem Boethius in »dieser beachtenswerten Kritik an der stoischen Abbildtheorie ${ }^{88}$ die Produktivität des menschlichen Denkens $\ll^{89}$ herausarbeitet, liefert er den scholastischen Debatten um die Erkenntnisfähigkeit der Seele ein Konzept, das dort weithin Wirkung entfaltete. Im 13. und 14. Jahrhundert ist die Philosophie intensiv mit dieser Konzeption befasst. ${ }^{90}$ Zwei sich überlagernde Problemkomplexe entfalteten ihre Wirkung: zum einen das Bild vom Weg der Erkenntnis von den Wahrnehmungen, bei denen sie beginnt, zu den Einsichten, auf die sie zielt, zum anderen das Verhältnis von passiver Affiziertheit und aktiver Denkleistung. Zusammen spannen sie eine Konzeption auf, in deren Mittelpunkt jeweils das >Obiectum< steht.

Entscheidend für die langfristige Entwicklung des Objektivitätskonzepts wird dabei die Semantik sein, die Boethius am Rande anklingen lässt: das Innere und das Äußere. Der Zielpunkt (obiectum), auf den sich Erkenntnis richtet, liegt in der Außenwelt, der Austragungsort, in dem sich Erkenntnis vollzieht, ist die innere Welt. Das christliche Denken hatte - mit großer

88 Die zitierte Passage schließt an einen poetischen Textteil an, eines der Cedichte (Carmina), mit denen Boethius die prosaischen Textteile versetzt: »Einstmals brachte der Stoa Kreis/ Alte, neblige Männer hervor,/Die da meinten, es sei dem Geist/Von den Körpern von außen her/Bild und Sinne so aufgeprägt,/Wie der emsige Griffel oft/Auf die ebenen Tafel, die/ Noch von Zeichen nicht eine Spur/Auf sich trägt, seine Lettern setzt./Doch wie drückte lebendger Geist/ohne eigne Bewegung sich aus,/Wenn er selbst nur geduldig liegt,/ Sich dem Eindruck der Körper fügt,/Wenn er tot wie ein Spiegelglas/Gibt ein Abbild der Außenwelt? Woher käme das Wissen dem Geist,/Das ihn stark macht, das All zu schaun?/ Wo die Kraft, die das Einzelne sieht/Und die aufteilt, was sie erkennt,/Das Ceteilte von neuem eint,/Daß sie wechselnd die Wege wählt,/Jetzt dem Höchsten das Haupt gesellt,/ Jetzt zum Tiefsten heruntersteigt,/Wieder dann zu sich selber kehrt/Und mit Wahrheit das Falsche besiegt?/Dies ist der mächtig bewirkende Grund,/Weit über jenen andern hinaus,/Der in der Art des trägen Stoffs/Jeden Eindruck nur duldend trüg,/Freilich geht ihr erregend vor,/Was des Geistes Kräfte bewegt:/Lebender Körper Empfänglichkeit,/Wenn das Licht in die Augen fällt/Und die Stimme im Ohr schallt./Dann erweckt auch des Geistes Kraft,/Was an innerer Schau er trägt, Ruft zu gleicher Bewegung auf, Paßt es äußerem Eindruck an/Und vermählt im Innern nun/der verborgenen Form das Bild. «195f. Boethius führt hier die aktive Geistestätigkeit des dialektischen Unterscheidens (siehe Kapitel 14: Kritik) so ins Feld, dass durch diese methodische Tätigkeit die geistigen Formen aktiv auf die empfangenen Eindrücke angewandt werden (die Übersetzung ist hier recht frei, im Lateinischen lesen wir: »Tum mentis vigor excitus/quas intus species tenet/ad motus similis vocans/notis applicat exteris/introrsumque reconditis/formis miscet imagines.«

89 Flasch, 1982, S. 108.

90 Vgl. Historisches Wörterbuch der Philosophie, hrsg. v. Joachim Ritter, 〉Objekt<, Bd. 6, insbes. 1026-1035. 
Wucht schon bei Augustinus (354-430) - im Unterschied zum antik-griechischen Denken den Selbstbezug des Menschen als Hinwendung auf die eigene Seele, also auf einen emphatisch aufgeladenen Innenraum, konzipiert. ${ }^{91}$

Das Begriffspaar Subjekt/Objekt bildet sich auf dieser Grundlage ebenfalls in der Scholastik aus. Boethius hatte das aristotelische $>$ Zugrundeliegende< (hypokeimenon) mit ssubiectum übersetzt und damit (gemäß der aristotelischen Kategorienschrift) das Verhältnis von Wesen (Substanz) und zufälligen Eigenschaften (Akzidenz) bestimmt: Eigenschaften können nur in einem Zugrundeliegenden (subiectum), einer Substanz auftreten. Subjekt bezeichnet also Substanz als Träger von Eigenschaften. Entsprechend bedeuten die Adjektive subiektive/obiektive in der Hochscholastik zwei Weisen oder Stadien, in denen etwas im Intellekt bzw. der Seele sein kann: als Erkenntnis dessen, was einem Träger akzidentell zukommt (wie das weiß einer Oberfläche) (»Esse in intellectu subiective est inhaerere ipsi, sicut accidens suo subiecto, ut albedo superficiei«) und als Erkenntnisakt, wie er sich vollendet. (»Esse in intellectu obiective est terminare actum intellectus $)^{92}$

Obwohl bis weit in die moderne Philosophie die Unterscheidung von Trägersubjekt (mitsamt zukommender Eigenschaften) und Zielobjekt des Erkenntnisakts erhalten blieb, wurde mehr und mehr das Moment des Zufälligen im Verhältnis von Trägersubstanz und Akzidenz semantisch wirksam, zumal das zugrundeliegende teleologische (zielorientierte) Erkenntnismodell in der Neuzeit durch ein mechanistisches ersetzt wurde - was die Beziehung von Geist zu Erkenntnisgegenstand aus der vormaligen Integration löste und einem fundamentalem Zweifel aussetzte (Descartes). In diesem neuen Paradigma konnte das Subjektive bald als Ort willkürlicher Zuschreibung, das Objektive als Instanz eines begründeten Urteils aufgefasst werden - so etwa beim Jesuiten Alfonso Antonio de Sarasa (1618-1667). Dort heißt es:

»Diejenige Gewißheit, die aus einer positiv vorliegenden Evidenz oder aus Gründen entspringt, welche hinreichend sind, damit man rationalerweise

91 Birgit Criesecke: Der Innenraum der Erfahrung. Selbstbezug, Selbstobjektivierung und die Genese des Selbstexperiments. In: Nicolas Pethes/Birgit Criesecke/Marcus Krause/ Katja Sabisch: Menschenversuche. Eine Anthologie 1750-2000, Frankfurt a.M. 2008, S. 37-44 (mit Rekurs auf Werner Kogge: Begreifen von Erfahrung. Über den Verlust der Erfahrung in der Geschichte des Denkens, unveröffentl. Manuskript eines Vortrags in Leipzig 2007).

92 Zitiert nach Historisches Wörterbuch der Philosophie, hrsg. v. Joachim Ritter, sSubjekt/ Objekt, subjektiv/objektiv«: Bd. 10, S. 401; mit unklarer Quellenangabe. 


\section{BASISWISSEN 9: Was bedeutet `Objektivität‘?}

Objektivität steht so sehr für Wissenschaftlichkeit, dass >objektive Wissenschaft fast schon nach einer Verdopplung klingt. Wissenschaft objektiviert, was subjektiv empfunden oder gemeint ist. So jedenfalls wird heute gedacht und gesprochen. Doch welcher Gedanke steckt in diesen Worten?

Die Wissenschaftsgeschichte rekonstruiert für das 18. und 19. Jahrhundert, wie eine ontologische Auffassung von Objektivität durch moderne Konzepte von Objektivität ergänzt und abgelöst wurde. Doch >ontologisch trifft den historischen Kern des Begriffs nicht: vielmehr als um einen Begriff der Seinslehre handelt es sich um einen der Erkenntnistheorie.

Der Begriff Objekt stammt aus einer Interpretation der antik-griechischen Erkenntnistheorie durch Boethius (ca. 480-526). Mit Objekt wird gemäß dieser Interpretation das Strebensziel des Erkennens bezeichnet, und zwar als ein Gegenüber (wörtlich: Entgegengeworfenes; Entgegenstehendes), das gegenüber den im Intellekt befindlichen Auffassungsformen äußerlich ist.

Das Begriffspaar objektiv/subjektiv wurde in der Hochscholastik gebildet, indem der Ausrichtung auf das Strebensziel (auf das Objekt gerichtet: objektiv) die Trägersubstanz gegenübergestellt wurde, deren Eigenschaften $\mathrm{zu}$ bestimmen sind. Als Bestimmungsgrund hieß die Trägersubstanz schon bei Boethius Subjekt (Untenstehendes; übers. v. griech. hypokeimenon) und so wurde das Zu-Bestimmende, wie es im Intellekt zunächst gegeben ist, zum Subjektiven.

urteilen kann, daß es sich tatsächlich so verhält, wie es sich dem Ceist darstellt, ist für >objektiv<, d.h. dem Objekt entsprungen, zu betrachten [...] Die andere Cewißheit stammt aus dem Subjekt. Sie liegt vor, wenn einer irgendeiner Meinung oder These verbissen anhängt [...] Diese Cewißheit [...] rührt 
Diese semantische Konstellation bildet die Grundlage für die moderne Auffassung von Objektivität. Das Konzept der Objektivität prägt sich als aperspektivische (auch: soziale) und als mechanische (auch: methodische) Konzeption von Objektivität aus. Aperspektivische oder soziale Objektivität ist mit der Überwindung individueller Perspektive durch Pluralisierung der `Standpunkteく, durch die Herstellung von Öffentlichkeit, durch wissenschaftliche Professionalisierung und Verfahren der Prüfung in der scientific community verknüpft; mechanische (auch: methodische) Objektivität mit der Automatisierung von Wahrnehmung und Datenverarbeitung, also mit dem Einsatz von Messinstrumenten, Quantifizierung und mathematischen Verfahren. (Daston 1992; Heintz 2000; Daston/Galison 2007)

Die mit dem Begriff Objektivität verbundene Validierung ließ sich sodann im Zeichen der Kritik (siehe Kapitel 13) weiter ausführen: Objektivität ist demnach nur so weit erreichbar ist, wie sich das Subjekt ebenso einer kritischen Prüfung unterzieht. (Harding 1995)

Lorraine Daston: Objectivity and the Escape from Perspective. In: Social Studies of Science, Vol. 22, No.4. 1992. S. 597-618.

Lorraine Daston/Peter Galison: Objektivität. Frankfurta.M. 2007.

Bettina Heintz: In der Mathematik ist ein Streit mit Sicherheit zu entscheiden. Perspektiven einer Soziologie der Mathematik. In: Zeitschrift für Soziologie, No. 5 (2000), S. 339-360.

Sandra Harding: >Strong Objectivity : A Response to the New Objectivity Question. In: Synthese,3 (1995), S. 331-349.

offensichtlich aus dem Subjekt selber her, d.h. aus dem sich Gewißheit fabrizierenden Menschen «. ${ }^{93}$

Wir befinden uns in der Mitte des 17. Jahrhunderts. Wenn die Wissenschaftsgeschichte für das 18. und 19. Jahrhundert eine aperspektivische (auch: so-

93 Zitiert nach Historisches Wörterbuch der Philosophie, hrsg. v. Joachim Ritter, sSubjekt/ Objekt, subjektiv/objektiv«: Bd. 10, S. 404. 
ziale) und eine mechanische (auch: methodische) Konzeption von Objektivität unterscheidet, so waren die Grundmotive von Objektivität in Abgrenzung von subjektiver Willkür und subjektiver Privatheit also längst vorbereitet.

Was die wissenschaftsgeschichtlichen Beobachtungen aber zeigen, ist, wie auf dieser Grundlage Objektivität immer mehr zur Verfahrensform wurde: Eine mechanische Erfassung des Gegenstandes durch Einsatz von Messinstrumenten, Quantifizierung und standardisierten Verfahren wurde zum Ideal; Sozialität durch wissenschaftliche Professionalisierung und durch Verfahren der Prüfung in der scientific community wurde zur Bedingung. Dass in diesem Umschlag in die Verfahrensform, dass im Namen von Objektivität die Sache, der es gerecht zu werden gälte, leicht aus dem Blick geraten kann - das liegt auf der Hand. Objektivität ohne Affektion durch das `Zielobjekt< des Forschens kann ihrerseits in Willkür umschlagen.

Dies ist der Einsatz einer reflexiven Objektivität im Zeichen des Paradigmas der Kritik (siehe Kapitel 13). Objektivität ist demnach nur so weit erreichbar, wie sich das Subjekt ebenso einer kritischen Prüfung unterzieht. ${ }^{94}$

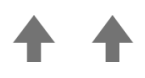

\section{Der "Sehepunkt» des Verstehens - das Paradigma der Kritik: Wissenschaftlichkeit durch Reflexivität}

Wenn die Beweislehre wissenschaftstheoretisch die prominenteste Traditionslinie der Wissenschaftskonzeptionen ist, so ist die der Kritik die verborgenste - und dennoch ist sie in der Praxis ebenso relevant. Im Kern geht es in dieser Traditionslinie darum, im schon Bekannten die Bedingungen, Merkmale und Konstitutionsmomente herauszuschälen, die für ein Phänomen entscheidend sind.

94 Vgl. Sandra Harding: Whose Science? Whose Knowledge? Thinking from Women's Lives, Ithaca 1991 (deutsch: Das Geschlecht des Wissens: Frauen denken die Wissenschaft neu, Frankfurt a.M. 1994); Sandra Harding: sStrong Objectivity«: A Response to the New Objectivity Question. In: Synthese, 3(1995), S. 331-349. 
Der Gedanke, dass Wissenschaft letztlich darin besteht, jede >Erkenntnis kritisch (von griechisch krinein: unterscheiden) daraufhin $\mathrm{zu}$ befragen, ob sie tatsächlich Bestand hat, dieser Gedanke ist der vielleicht am tiefsten verankerte, aber am wenigsten bewusste Gedanke zur Bestimmung von Wissenschaftlichkeit. Von der Platonischen Formel gnosthenta krinai (das Eingesehene unterscheiden) ${ }^{95}$ über die Aristotelische Konzeption eines Unterscheidungsvermögens als Kern der Erkenntnisfähigkeit ${ }^{96}$ und reflexiven Klugheit, ${ }^{97}$ über die Stoa, Cicero und Quintilian in die Neuzeit zu Petrus Ramus wird in bemerkenswerter Kontinuität diese Denkfigur entfaltet.

Ihren eminenten Stellenwert erhielt die Kritik dadurch, dass sie als Methodik der beiden grundlegenden Erkenntniswege der antiken Philosophie angesehen werden konnte. Sie liegt sowohl im Kern der Platonischen Dialektik mit ihrem Abschälverfahren als auch in dem der Aristotelischen Analysis, die ebenfalls auf Unterscheidungsfähigkeit aufbaut. Auf dieser Basis konnten z.B. die Stoiker Dialektik und Kritik identifizieren und von der Grammatik so absetzen, dass es in der Kritik wie schon bei Platon um kritische Reflexion von Gedachtem, nämlich »um die inhaltliche Beurteilung des von der Grammatik festgestellten Textes geht. ${ }^{98}$

In Reaktion darauf wiederum konnte Cicero - die Einteilung des aristotelischen Organons aufnehmend - gegen eine (unterstellte) Fixierung auf den Weg der Kritik (»via iudicandi«: »die Wissenschaft (scientia), die Dialektik genannt wird «) eine ars inveniendi (Erfindungskunst) im Sinne der Topik einfordern. ${ }^{99}$ Dieser Gegensatz von Kritik und Erfindungskunst wurde über Boethius und die Scholastik tradiert und blieb auch in der frühen Neuzeit

95 Platon: Politikos (Übersetzung von Friedrich Schleiermacher). Sämtliche Werke in zehn Bänden, Frankfurt/Leipzig 1991, 259c.

96 Aristoteles: Über die Seele, auf Grundl. d. Übers. v. W. Theiler hrsg. v. Horst Seidl Hamburg 1995, 432a 16. Vgl. zu Platon und Aristoteles den Eintrag ’Kritik« in: Historisches Wörterbuch der Philosophie, hrsg. v. Joachim Ritter, Bd. 4, insbes. S. 15195-15199.

97 Aristoteles: Nikomachische Ethik, übers. u. hrsg. v. Ursula Wolf, Reinbek bei Hamburg 2006, 1143a.

98 Eintrag «Kritikı in: Historisches Wörterbuch der Philosophie, hrsg. v. Joachim Ritter, Bd. 4, insbes. S. 15206.

99 Vgl. dazu: Historisches Wörterbuch der Philosophie, hrsg. v. Joachim Ritter, Bd. 4, S. 15207. Quintilian konnte mit revidierendem Rekurs auf Cicero resümieren: »Denn in jenen [den dialektischen Unterredungen] suchen gelehrte Menschen unter Gelehrten nach dem Wahren, durchforschen alles bis ins Kleinste und mit ängstlicher Genauigkeit und führen es zur Gewißheit und zum allgemein Zugestandenen, so daß sie für sich sowohl den Teil 
intelligibel, sodass im 16. Jahrhundert Petrus Ramus an die antike Denkfigur der sunterscheidenden Beurteilung von Eingesehenem formulieren konnte, dass »wir natürlicherweise zuerst gedanklich erfassen, was erörtert werden soll, und dann dieses Angeordnete unterscheidend behandeln ${ }^{100}$

Doch - wie unterscheiden wir eigentlich? Dieser Frageaspekt trat in den Vordergrund, als in der Neuzeit der Gang der Erfahrung desto prekärer erschien, je mehr man ihrer jeweiligen ssubjektiven Bedingungen gewahr wurde. Eine Ausprägung dieses Gewahrwerdens lässt sich im englischen Empirismus beobachten. So hat John Locke programmatisch die Aufgabe bestimmt, die »Betrachtung der Ideen und Wörter als der hauptsächlichsten Hilfsmittel der Erkenntnis« so "gründlich« voranzubringen, dass »sie uns vielleicht eine andere Art von Logik und Kritik liefern als bisher bekannt ist. ${ }^{101}$ Von da aus spitzte sich die Frage nach den Erkenntnisbedingungen $\mathrm{zu}$, und zwar über Hume bis hin zu Kant, für den das Wort Kritik titelgebend wurde für seine Hauptschriften. Eine Kritik der Vernunft zu schreiben, bedeutete für Kant, »ein System der Vorsicht und Selbstprüfungsberichte [der Vernunft], vor welchem kein falscher vernünftelnder Schein bestehen kann $\ll^{102}$ zu verfassen. Damit war der Gedanke der Kritik als spezifisches Verfahren etabliert: nämlich als Methode der Rückfrage auf die eigenen Grundlagen und Vorgehensweisen.

Parallel zu dieser erkenntnistheoretischen Entwicklung fand der Begriff der Kritik eine nähere Bestimmung in der philologischen Tradition. Tatsächlich waren beide Strömungen stets eng verflochten, denn sie standen in einem komplizierten Diskursgewebe zwischen Logik, Grammatik und Textauslegung miteinander in Verbindung. In der Textauslegung, also im Kontext der Verstehenslehren, ging es darum, zunächst einmal die Sachlage

der Findung wie den der Beurteilung in Anspruch nehmen, deren eine sie Topik, deren andere sie Kritik nennen.« (a.a.O. S. 15209).

100 Historisches Wörterbuch der Philosophie, hrsg. v. Joachim Ritter, Bd. 4, S. 15213-15217; Übers. wk.

101 John Locke führt die «Kritik« in seinem Kapitel »Über die Einteilung der Wissenschaften« unter der»Lehre von den Zeichen « (semeiotika) neben den physika (Naturwissenschaften) und den practica (Lehre von den menschlichen Handlungen; Ethik). Versuch über den menschlichen Verstand, Buch IV, Kap. XXI, Abs. 4, Hamburg 1988. Zitat auf S. 439.

102 Immanuel Kant: Kritik der reinen Vernunft 2. Werkausgabe Bd. 4, hrsg. v. Wilhelm Weischedel, Frankfurt a.M. 1974, S. 612 (A 711; B 739). 
zu sichern. So stellte etwa der Thesaurus Criticus (1602-1623) eine frühe Form dessen dar, was später philologische Kritik heißen würde, nämlich eine Vervollständigung, Korrektur und Kommentierung antiker Texte. ${ }^{103}$ Kritik bedeutet hier eine Prüfung von Texten und Quellen hinsichtlich dessen, wofür sie überhaupt stehen können; also welche Aussagen und Interpretationsansätze sie in Bezug auf angenommene Herkunft, Autorschaft, Zeit, Kontext und kulturellem Hintergrund überhaupt erlauben. Bis zum 19. Jahrhundert hatte sich diese Fragerichtung zur Wissenschaftsform der historischen Kritik ausgeprägt. Das lässt sich in aller Deutlichkeit wiederum bei Droysen nachlesen:

»Aus dem großen Aufschwung der Philologie, und namentlich nach der Seite der Altertumsstudien, ergaben sich die großen kritischen Forschungen [...] Ihr Wesen war, daß man die Erkenntnisquellen, aus denen die Kunde des Altertums zu erforschen war, zum Gegenstand der Forschung machte. [...] Die Ansicht ist hier, daß die historische Kritik den objektiven Tatbestand zu ermitteln habe, dass dieser aus der strengen Untersuchung und Vergleichung der Berichte zu gewinnen sei, daß die Kritik so die Berichte authentisch zu machen habe. «104

Die Wendung auf die Erkenntnisquellen erfolgte in diesem Bereich des Diskursgeflechtes nur etwas anders als im englischen Empirismus. Während dort das Subjekt als ein Apparat der Verarbeitung von Sinneswahrnehmungen hinsichtlich der Zuverlässigkeit dieses Verarbeitungsprozesses in Zweifel geriet, ${ }^{105}$ waren es hier die historischen und kulturellen Bedingungen, die als spezifische Bezogenheiten von Erkenntnis in den Blick kamen. Zunächst ging es noch um die persönlichen Lebensumstände, die bei der Interpretation zu berücksichtigen seien. Johann Martin Chladenius, dessen Einleitung zur richtigen Auslegung vernünftiger Reden und Schriften (1742) für die hermeneutische Tradition wichtig wurde, schreibt:

103 Vgl. Historisches Wörterbuch der Philosophie, hrsg. v. Joachim Ritter, Bd. 4, S. 15237.

104 Johann Gustav Droysen: Historik. Rekonstruktion der ersten vollständigen Fassung der Vorlesungen (1857), Grundriß der Historik in der ersten handschriftlichen (1857/1858) und in der letzten gedruckten Fassung. Textausgabe v. Peter Leyh, Stuttgart/Bad Cannstatt 1977, S. 113.

105 Thomas Hobbes: Leviathan, Frankfurt a.M. 1994, S. 14. 
»Diejenigen Umstände unserer Seele, Leibes und unserer ganzen Person, welche machen oder Ursach sind, dass wir uns eine Sache so und nicht anders vorstellen, wollen wir den Sehe-Punckt nennen«, diesen Sehe-Punkct sodann definierend als »der innerliche und äußerliche Zustand eines Zuschauers, insofern daraus eine gewisse und besondere Art, die vorkommenden Dinge anzuschauen und zu betrachten, fließt. «106

Spätestens hier war Idee des Perspektivismus geboren - und zwar aus dem Geiste der Kritik. Bei Schleiermacher lesen wir dann einige Jahrzehnte später:

»Der Sprachschatz und die Geschichte des Zeitalters eines Verfassers verhalten sich wie das Ganze, aus welchem seine Schriften als das Einzelne müssen verstanden werden, und jenes wieder aus ihm. ${ }^{107}$

Wir sehen also: Im Zusammenhang von erkenntnistheoretischer Radikalisierung und hermeneutischem Bewusstsein entsteht ein Gedanke, der die Berücksichtigung der Besonderheit und Unterschiedlichkeit von persönlichen und kulturellen Hintergründen zu einem zentralen Element ernsthafter Wissenschaft erklärte. Die Frage, wodurch Erkenntnis bedingt ist, lenkte den Blick sowohl auf die eigene Erkenntnisweise als auch auf die Differenz zwischen unterschiedlichen Bedingungen von Erkenntnis. Beides zusammen ergab dann das Konglomerat, aus dem sich im 19. Jahrhundert sowohl die Kritik des Marxismus (die klassenmäßigen Bedingungen des Erkennens) als auch der Perspektivismus Nietzsches (die triebmäßigen Bedingtheiten des Erkennens) speisten.

Im 20. Jahrhundert prägte sich das Grundmotiv der Kritik auf verschiedene Weisen weiter aus. Es findet sich früh schon entwickelt in der Phänomenologie Husserls und Heideggers mit ihrer Kernbotschaft, dass Erkenntnis mehr sei als Gegenstandswissen, nämlich ein Wissen um Gegenstände in der Art und Weise ihres Gegebenseins; es findet sich in der Kritischen Theorie, die im Wissen die Momente von Herrschaft herausstellt, in soziologisch und strukturalistisch gewendeten Wissens- und Wissenschaftstheorien etwa bei Ludwik Fleck, Thomas S. Kuhn und Michel Foucault, die zeigen, wie wissen-

106 Johann Martin Chladenius: Einleitung zur richtigen Auslegung vernünftiger Reden und Schriften (Leipzig 1742), Düsseldorf 1969, S. 309.

107 Schleiermacher, 1977, S. 95. 
schaftliche Erkenntnis stets innerhalb je spezifischer sozialer, praktischer und materialer Konstellationen steht; sie findet sich bei Autoren wie Maturana, Varela, Heinz von Förster und Niklas Luhmann, die, inspiriert von biologischen Konzepten, darlegen, wie Wissen in Systemen produziert wird (seien es biologische oder soziale); sie findet sich auch in den jüngst noch als avancierteste Form der Erkenntnistheorie geltenden Positionen der Postmoderne und `Dekonstruktion<, am deutlichsten in Jacques Derridas Ansatz, Denksysteme auf ihre in sich nicht mehr legitimierte Grundentscheidungen zurückzuführen und so ihrer letztgültigen Autorität zu berauben.

Eine besonders pointierte Auffassung zur Herangehensweise der Kritik formuliert der französische Philosoph und Wissenshistoriker Michel Foucault:

»Von der Romantik bis zur Frankfurter Schule wurde immer wieder die Rationalität mit dem ihr eigentümlichen Gewicht der Macht in Frage gestellt. Nun besteht die Kritik der Erkenntnis, die ich Ihnen vorlegen werde, nicht nur darin, das beständig [...] unterdrückende Moment der Vernunft anzuprangern, denn schließlich, glauben sie mir, ist die Unvernunft genauso unterdrückend. [...] Die Kritik, die ich Ihnen vorschlage, besteht darin zu bestimmen, unter welchen Bedingungen und mit welchen Auswirkungen sich eine Veridiktion vollzieht. [...] Das Problem besteht darin, die Bedingungen sichtbar zu machen, die erfüllt sein mußten, damit man über den Wahnsinn - aber dasselbe gilt für die Delinquenz und für die Sexualität - sprechen, Diskurse halten kann, die wahr oder falsch sein können, und zwar nach den Regeln der Medizin oder der Beichte oder, darauf kommt es nicht an, der Psychoanalyse.« ${ }^{108}$

Kritik als wissenschaftliche Methode in diesem Sinne ist durchaus auch gängige Praxis in Einzelwissenschaften. So beschreibt die Biologin Evelyn Fox Keller die Entwicklung in der modernen Molekularbiologie als den Vorgang einer konzeptuellen Verdeckung des Gegenstandes: »Der Gen-Diskurs hatte eine räumliche Karte, die das Zytoplasma vom wissenschaftlichen Standpunkt aus unsichtbar machte. ${ }^{109}$ Reflexion im Genre der Kritik findet

108 Michel Foucault: Die Geburt der Biopolitik Geschichte der Gouvernementalität II, Frankfurt a.M. 2006, S. 61.

109 Evelyn Fox Keller: Das Leben neu Denken. Metaphern der Biologie im 20. Jahrhundert, München 1998, S. 43. 
auch statt, wo es z.B. um »Missverständnisse um chemische Konzepte« oder um »Alternative Metaphors for Artificiel Intelligence " geht. ${ }^{110}$

Halten wir fest: Im Wissenschaftstypus der Kritik wird der Anspruch erhoben, einen strengeren, tiefgreifenderen und umfassenderen Wissenschaftsbegriff einzulösen, als es ein einfacher, nur auf den Erkenntnisgegenstand bezogener leisten kann, und zwar, indem die Bedingungen von Wissensbildung in den Erkenntnisprozess aktiv und reflexiv einbezogen werden.

\section{Noch einmal zum Beschreiben: Das Wissenschaftsideal der Deskription - Beobachten, Beschreiben, Tatsachen (Bezüge zu Empirismus und Positivismus)}

Einige wissenschaftliche Disziplinen tragen das Ideal der Deskription schon in ihrem Namen - etwa Geographie und Ethnographie (graphein: das griechische Wort für ritzen, schreiben). In vielen anderen Disziplinen ist das Deskriptive mehr eine Tiefenüberzeugung: Im Kern bestehe Wissenschaft in genauer Beobachtung, in der Feststellung von Tatsachen und in ihrer möglichst detailgetreuen Beschreibung. So sieht ein Bild von Wissenschaft aus, das vor allem auf nüchterne Betrachtung setzt und sich gegen jede Art von Spekulation wendet. Die klassischen Gegenüberstellungen sind die von Beschreiben versus Interpretieren und Beschreiben versus Erklären. Das Beschreiben will »Zurück zu den Sachen selbst! «111, es fordert: »Denke nicht,

110 Martin Jansen/Ulrich Wedig: Das Teil im Ganzen - Missverständnisse um chemische Konzepte. In: Angewandte Chemie: 2008, 120, S. 10176-10180; David M. West/Larry E. Teravis: From Society to Landscape. Alternative Metaphors für Artificial Intelligence. In: Al Magazine, 2 (1991), S. 69-83.

111 Dieses der phänomenologischen Philosophie zugeschriebene Motto findet sich bei Edmund Husserl als abgrenzenden Anschluss an den Empirismus, dem er eine Verwechslung von originärer Erfahrung und Natursachen vorwirft. Was anstelle eines naiven Sachbezuges aber gefordert ist, so Husserl: „sich nach den Sachen selbst richten bzw. von den Reden und den Meinungen auf die Sachen selbst zurückgehen, sie in ihrer Selbstgegebenheit befragen und alle sachfremden Vorurteile beiseitetun." Eine Selbstgegebenheit der Sachen >vor allem Diskurs anzunehmen, die allerdings als Gegebenheit nicht einfach >da< ist, sondern der Befragung bedarf, dies kann als der genuine Einsatz der philosophischen Phänomenologie gelten. Edmund Husserl: Ideen zu einer reinen Phänomenologie und phänomenologischen Philosophie (1913), Ges. Schriften Bd. 5, hrsg. v. Elisabeth Ströker, Hamburg 1992, (Husserliana Bd. III,1, V), S. $41 f$. 


\section{BASISWISSEN 10: Fakten und Daten}

Die Tradition der Naturalis Historiae hat als eine ihrer Ausprägungen eine Denkfigur hervorgebracht, die Wissenschaft als Feststellung, Sicherung und Beschreibung von Tatsachen bzw. Fakten betrachtet. Was hat es mit diesen - bei genauerem Hinsehen eigenartigen - Worten auf sich?

Fakten erscheinen im heutigen Sprachgebrauch als das sicherste und härteste im Wissen überhaupt. Bedenkt man aber die Herkunft des Wortes, nämlich das lateinische facere-machen, dann stellt sich die Frage, wie gerade etwas Gemachtes das sicherste Fundament von Wissen bilden soll. Plausibel wird dieser Zusammenhang aber dann, wenn wir nachvollziehen, dass die matter offacts, die ins Deutsche mit Tatsachen übersetzt wurden, als die Tatprodukte eines Schöpfergottes gedacht waren, also gerade als dasjenige, was, ohne dass es >notwendig zu sein bräuchte, als von einer übergeordneten Macht hergestellt schlicht besteht - gottgegeben sozusagen. (vgl. Historisches Wörterbuch der Philosophie, hrsg. v. J. Ritter, 10, 42.233) Als solche Gegebenheiten erscheinen Fakten als das Rohmaterial (brute facts) von Erkenntnis, das noch keiner intellektuellen und interpretativen Transformation unterzogen, noch keinem sallgemeinen Gesetz« subsumiert wurde. Und wenn Wissenschaft die Welt - im Gegensatz zu allen autoritären und spekulativen Deutungen - realistisch darstellen soll, dann kann es plausibel erscheinen, sie auf die Identifizierung und Zusammenstellung von Fakten bzw. Tatsachen verpflichten zu wollen.

Was aber sind Daten? Das Wort ist ein eingedeutschter Plural von lateinisch datum, das Partizip Perfekt von dare, ein Verb mit der Bedeutung geben. Seit dem 18. Jahrhundert hat sich daraus in der Wissenschaftssprache die Bedeutung gegebene Größe, Angabe, Beleg herauskristallisiert (vgl. Eintrag >Datum in: Etymologisches Wörterbuch des Deutschen, A-G, Akademie Verlag Berlin 1989). In diesem Kontext wurden Daten im 20. Jahrhundert zum wissenschaftsphilosophischen Problem. Es wurde argumentiert, dass Daten ebenso wenig wie brutefacts schlichtes Rohmaterial sind, sondern stets unter Bedingungen erhobene Daten. Man sprach von einem Myth of the Given (Sellars: Empiricism and the Philosophy of Mind, 1956) und diskutierte die Theoriegeladenheit (Theory-ladenness) der Beobachtung (Hanson: Patterns of Discovery, 1958). 
sondern schau! «112 So drückt sich diese Auffassung im philosophischen Denken zu Beginn des 20. Jahrhunderts aus, und zwar in zwei Ansätzen, die im Beschreiben eine besonders disziplinierte und kritische Form von Erkenntnis erblickten. Insofern ist das Beschreiben eng verwandt mit den Positionen der Kritik, die wir im letzten Abschnitt behandelt haben; und durchaus auch mit dem Paradigma der Adaequation, das wir uns davor angeschaut haben: Nur, dass die Annäherung an den Gegenstand gerade nicht durch eine fortgesetzte Anpassung der Interpretation, sondern - im Gegenteil - durch eine weitestmögliche Rücknahme von Interpretation erfolgen soll; eine entgegengerichtete Geste also.

Grundzüge einer faktenbezogenen Wissenschaftsauffassung waren für zwei philosophische Strömungen maßgeblich, die die Wissenschaftstheorie im 19. und frühen 20. Jahrhundert prägten: Empirismus und Positivismus. Doch aus beiden Strömungen gingen sehr heterogene, ja, in manchen Aspekten sogar widersprüchliche Überzeugungen in unsere heutigen Begriffe von Wissenschaft ein. Die Ausdrücke empirische Wissenschaft und positive Wissenschaft implizieren sicherlich Ideale von Wissenschaftlichkeit. Doch was für welche? Die Geistesgeschichte hat in diesen Begriffen einige Verwicklungen produziert, deren Fäden wir auseinanderziehen müssen, um Klarheit über diese Konzeptionen zu gewinnen.

Packen wir das Problem an der Wurzel: Unser Begriff Empirie geht zurück auf das griechische Wort empeiría, das, wie erläutert (Abschnitt BASISWISSEN 6: Induktion und Empirie) bei Aristoteles eine zentrale Rolle spielt.

112 Ludwig Wittgensteins Spätphilosophie kann insgesamt als deskriptive Wende der Philosophie verstanden werden. In diesem Kontext steht die Forderung: »Denke nicht, sondern schau! (Ludwig Wittgenstein: Philosophische Untersuchungen. In: Werkausgabe Bd. 1, Frankfurt a.M. 1982, § 66). Konzepte und Begriffsordnungen werden hier nicht mehr als intellektuell unmittelbar zugängliche Ordnungen verstanden (»denke nicht!«), sondern als beschreibbare soziale Praktiken. Durch Untersuchung der Sprachpraxis zeigt sich z.B. das Feld der sSpiele` als ein durch Familienähnlichkeiten gewobener Zusammenhang von Fällen, dessen Strukturen in einer Untersuchung der Sprachpraxis sichtbar werden (schau!). Dass es dabei nicht um die empirisch vorfindliche, sondern um die durch strukturelle Analogien angelegte Sprachpraxis geht, wird in Teilen der Wittgenstein-Forschung zu wenig berücksichtigt. Vgl. Werner Kogge/Birgit Criesecke: Ein Arbeitsprogramm, kein Abgesang. Wittgensteins grammatische Methode als Verfahren experimentellen Denkens. In: Stefan Tolksdorf/Holm Tetens: In Sprachspiele verstrickt, oder, Wie man der Fliege den Ausweg zeigt: Verflechtungen von Wissen und Können, Berlin/New York 2010, S. 101-126. 
Übersetzt wurde dieses Wort ins Lateinische mit experientia und experimentum - Worte, die im Deutschen sowohl mit Erfahrung als auch mit Experiment wiedergegeben worden sind. Hier müssen wir genauer hinsehen und Bedeutungsunterschiede festhalten, die in der modernen Wissenschaftstheorie häufig übergangen werden. In der Wissenschaftstheorie kennt man nämlich Erfahrung seit der frühen Neuzeit lediglich als Sinneswahrnehmung oder als künstlich eingerichtetes Experiment. Die aristotelische empeiría ist aber etwas Drittes. Empeiria ist für Aristoteles ein eigenständiges Potenzial, das sich im Menschen naturwüchsig, durch sein erprobendes Tätigsein, ausprägt. Älter zu werden bedeutet zumindest potentiell, Unterscheidungsfähigkeit zu gewinnen. Aristotelisch gedacht wäre eine Erfahrungswissenschaft also eine, die durch die Erfahrenheit ihrer Beteiligten geprägt ist (ein Gedanke, der in der Wissenschaftsforschung seit Luwik Fleck und Michael Polanyi mit ihren Überlegungen zu Denkstil und Tacit Knowledge durchaus aktuell ist).

Doch der historische Empirismus, der sich seit dem 17. Jahrhundert ausprägte, ist völlig anders gestrickt: Er kehrt das Muster des aristotelischen Erfahrungsprozesses an entscheidender Stelle um. Der Empirismus ist einerseits mit dem Ideal der unmittelbaren sinnlichen Wahrnehmung verbunden, zugleich liegt im Moment seines Entstehens der radikalste Zweifel an der Möglichkeit von Erkenntnis aus sinnlicher Wahrnehmung. Das bedeutet im Resultat: Aus der sinnlichen Wahrnehmung kann die Erkenntnis nur das verarbeiten, was festgehalten, gesichert, am besten sogleich überführt werden kann in ein System zeitloser Einheiten und Relationen. Aus dem Leiden an der Sinnlichkeit entsteht so die Leidenschaft für formale Logik und Mathematik. Empirismus profitiert zwar noch von seinem nominellen Bezug zu Erfahrung, tatsächlich aber ist der sinnliche Bezug zur Materialität des Gegenstandes nur noch Apercu; der Empirismus zeigt sich somit als Begleitideologie der Mathematisierung (und es sind erst die jüngeren Strömungen der Wissenschaftsforschung und Experimentaltheorie, die das aristotelische Moment der Empirie wieder aufrufen).

Eng verbunden mit der Geschichte des Empirismus ist die Entwicklung des Positivismus. Beide Strömungen beziehen ihr Renommee zwar von der scheinbaren Nähe zu Tatsachen und Fakten, propagieren in der Tat aber massiv die Übersetzung ins mathematische Medium, an Stelle einer Annäherung an das Untersuchungsmaterial - wie ein vertiefender Blick zeigt. 


\section{VERTIEFEND Empirie im Empirismus und Positivismus}

$\mathrm{Zu}$ besichtigen ist dies gleichsam in Echtzeit bei einem der ersten Vertreter des Empirismus, bei Thomas Hobbes. Sehen wir uns die einschlägige Textstelle in Hobbes Hauptwerk, dem Leviathan, an. Wie für Aristoteles beginnt für Hobbes die Erfahrung mit der Sinneswahrnehmung. Und genau wie Aristoteles bestimmt Hobbes Erfahrung als »[v]iel Erinnerung oder Erinnerung an viele Dinge«. Doch anders als bei Aristoteles entsteht aus dieser Erinnerung keine sich anreichernde Erfahrenheit - im Gegenteil: Hobbes denkt Erinnerung als »zerfallende Empfindung« im Sinne eines Abbaus: »[D] ie ständige Veränderung des menschlichen Körpers zerstört bald die Teile, die bei der Empfindung bewegt worden waren «. ${ }^{113}$ So führt für Hobbes zwar Erfahrung zu »Klugheit ${ }^{114}$ - so weit folgt er Aristoteles - Klugheit aber steht für Hobbes - anders als die aristotelische phrónēsis - außerhalb eines wissenschaftlichen Ansprüchen genügenden Wissens: »Die Zeichen der Klugheit sind alle unsicher «, schreibt er, »denn es ist unmöglich, durch Erfahrung $\mathrm{zu}$ beobachten und sich an alle Umstände zu erinnern, die einen anderen Ausgang bewirken können. ${ }^{115}$ Wissenschaft baut daher bei Hobbes gerade nicht auf Erfahrung, sondern darauf, durch »exakte Definitionen ${ }^{116}$ ein quasi-mathematisches System des logischen Schließens in Gang zu setzen.

Sehen wir uns nun die Entwicklung des Positivismus an, die eine sehr ähnliche Wendung nimmt wie die des Empirismus.

Worum geht es? 1876 schreibt Gustav Kirchhoff in der Vorrede zu seinen Vorlesungen über Mathematische Physik: Ich stelle »es als Aufgabe der Mechanik hin, die in der Natur vor sich gehenden Bewegungen zu beschreiben, und zwar vollständig und auf die einfachste Weise zu beschreiben. Ich will damit sagen, dass es sich nur darum handeln soll, anzugeben, welches die Erscheinungen sind, die stattfinden, nicht aber darum, ihre Ursachen zu ermitteln. $\aleph^{117}$ Dieses Ideal einer möglichst einfachen und klaren Deskription physikalischer Phänomene, die ausdrücklich auf die Vorstellung zugrundeliegender Ursachen verzichtet, fand so prominente Anhänger wie Heinrich

113 Thomas Hobbes: Leviathan, Frankfurt a.M. 1994, S. 14.

114 Hobbes, 1994, S. 37.

115 Hobbes, 1994, S. 38.

116 Hobbes, 1994, S. 38.

117 Gustav Kirchhoff: Vorlesungen über mathematische Physik. Mechanik, Leipzig 1876, S. III. 
Hertz ${ }^{118}$ und hatte so berühmte Vorläufer wie Ernst Mach. Den Grundgedanken, »den Blick von den bewegenden Ursachen« abzuwenden, »um einzig und allein die hervorgebrachte Bewegung zu betrachten« und die »dem Körper inhärierenden Kräfte« als »dunkle, der Metaphysik angehörigen Begriffe $\ll^{119} \mathrm{zu}$ verbannen, hatte aber auch schon Jean d'Alembert, einer der Begründer des Positivismus, ${ }^{120} 150$ Jahre früher vermerkt.

Ernst Mach schildert sehr anschaulich wie der Versuch, in der Physik ohne den Kraftbegriff auszukommen, motiviert ist.

»Betrachten wir ein magnetisches Stahlstück neben einem sonst gleich beschaffenen unmagnetischen. Während letzterer sich gegen Eisenfeile gleichgültig verhält, zieht ersterer dieselbe an. Auch wenn die Eisenfeile nicht vorhanden ist, müssen wir das magnetische Stück in einem anderen Zustand denken als das unmagnetische. Denn, dass das bloße Hinzubringen der Eisenfeile nicht die Erscheinung der Anziehung bedingt, zeigt ja das andere, unmagnetische Stück. Der naive Mensch, dem sich zur Vergleichung sein eigener Wille als bekannteste Kraftquelle anbietet, denkt sich in dem Magnet eine Art Ceist. «121

Mach schildert weiter, wie dann in einer ersten wissenschaftlichen Sichtweise aus der dämonischen Kraft eine Art Stoff wird, dessen Bewegung das Phänomen hervorbringt, bis sich schließlich die indirekte Beschreibung (z.B. über dämonische und stoffliche Ursachen) in eine direkte Beschreibung überführen lässt, »welche nichts Unwesentliches mehr enthält, und sich lediglich auf die begriffliche Fassung der Tatsachen beschränkt.«122 Und er wagt die Prognose: »Es zeigt sich hier deutlich der Weg, auf dem sich eine allgemeine,

118 Heinrich Hertz: Die Prinzipien der Mechanik, Leipzig 1894.

119 Jean d'Alembert: Abhandlung über Dynamik; in welcher die Cesetze des Cleichgewichts und der Bewegung der Körper auf die kleinstmögliche Zahl zurückgeführt und in neuer Weise abgeleitet werden, und in der ein allgemeines Princip zur Auffindung der Bewegung mehrerer Körper, die in beliebiger Weise aufeinander wirken, gegeben wird, Übers. u. Anm. v. A. Korn, Thun/Frankfurt a.M. 1997 [im Orig. 1743], S. 13.

120 Vgl. Georg Misch: Zur Entstehung des französischen Positivismus, Darmstadt 1969.

121 Ernst Mach: Die Vergleichung als wissenschaftliches Princip. In: Die Principien der Wärmelehre. Historisch-kritisch entwickelt von Ernst Mach, Leipzig 1896 (3. Aufl. 1919), S. 396-405, hier S. 400.

122 Mach, 1896, S. 401. 
alle Gebiete umfassende physikalische Phänomenologie, eine hypothekenfreie Darstellung der Physik entwickeln wird. ${ }^{123}$ Mach selbst hat ein solches Programm in gewisser Weise verfolgt - andere Vertreter der positivistischen Physik und die Hauptströmung der Wissenschaftstheorie allerdings bewegten sich in Richtung dessen, was Mach in Bezug auf Hertz bemerkt: "Die Physik gewöhnt sich allmählich ohnehin, die Beschreibung der Tatsachen durch Differentialgleichungen als ihr eigentliches Ziel anzusehen«, wodurch »die allgemeine Anwendbarkeit der Hertzschen mathematischen Aufstellung anerkannt [ist], ohne daß man sich auf weitere Interpretation der Kräfte oder Verbindung einlassen müsste. ${ }^{124}$ So war die positivistische Konzeption auch schon bei d'Alembert angelegt, der »insbesondere die Mechanik auf die mathematische Beschreibung der Wirkungen einschränkt, ohne auf bewegende Ursachen einzugehen«; mit dieser Darstellung, »welche die auf Phänomene reducierte Erkenntniss weiter einschränken auf Erfassung blosser Abhängigkeitsverhältnisse rein logischer Art [...] ist der Standpunkt des Positivismus gegeben.« ${ }^{125}$

Wir sehen also: In der Physik konnte die Restriktion auf Beschreibungen und Tatsachen so gedeutet werden, dass sie ein möglichst sparsames Set an mathematisch-logischen Formen zum Ausgangspunkt nahm. Damit schlägt auch der Positivismus um zum Paradigma der apodeixis in seiner mathematisierten Form. Tatsachen zeigen sich in Abhängigkeit und Funktion einer forciert reduzierenden mathematischen "Aufstellung«, sie selbst sind nicht das Ziel der wissenschaftlichen Bemühung.

Ebenso wie der Begriff der empirischen Wissenschaft bezeichnet auch jener der positiven Wissenschaft gerade nicht die Hinwendung auf den untersuchten Gegenstand in seiner phänomenalen Materialität, sondern deren Reduktion auf eine mathematisch >beschreibbare< Form - und das heißt in diesem Fall: auf eine Formalisierung. Das Paradigma der Beweislehre erscheint hier nur in einem neuen Gewand.

123 Mach, 1896, S. 403.

124 Ernst Mach: Die Mechanik in ihrer Entwicklung. Historisch-kritisch dargestellt. Hrsg. u. m. einem Anh. vers. v. Renate Wahsner u. Horst-Heino von Borzeszkowski, Berlin 1988 [1883], S. 285.

125 Misch, 1969, S. 36. 
Doch der Wissenschaftstypus des Beschreibens prägte durchaus eine eigene Form aus: Sie zeigt sich in all den Verfahren, die den gegebenen Sachverhalt nicht reduktiv in Bezug auf eine vorgegebene Theorieform, sondern möglichst umfassend aufzuzeichnen und zu sichern versucht. Es wurde bemerkt, dass Wissenschaft analog zur kriminalistischen Spurensicherung verfährt, wenn sie in einem Feld möglichst viele Details wahrzunehmen und aufzuzeichnen sucht. ${ }^{126}$ Das Beschreiben geht dabei in zwei Richtungen: in Richtung Fülle und Anspruch aufVollständigkeit und in Richtung Sicherung und Verdauerung. Beide Motive greifen Hand in Hand.

Wie die wissenschaftliche Praxis des Beschreibens sich auf den Aspektreichtum eines Phänomens richten kann, deutet sich z.B. in Formulierungen zur Konzeption einer Dichten Beschreibung beim Ethnographen Clifford Geertz an. ${ }^{127}$ Er notiert in Bezug auf Bedingungen der Wissenschaftlichkeit der Ethnographie:

»Zunächst einmal muss die Theorie [hier] näher am Boden der Tatsachen bleiben, als dies sonst bei Wissenschaften der Fall ist, die sich eher der imaginativen Abstraktion überlassen können. [...] Darin liegt die erste Bedingung für die Kulturtheorie: sie ist nicht ihr eigener Herr. Da sie von den unmittelbaren Momenten der dichten Beschreibung nicht zu trennen ist, bleibt ihre Möglichkeit sich nach Maßgabe einer inneren Logik zu formen, ziemlich beschränkt. Die Allgemeinheit, die sie möglicherweise erreicht, verdankt sich der Cenauigkeit ihrer Einzelbeschreibungen, nicht dem Höhenflug ihrer Abstraktionen. « ${ }^{128}$

Allgemeine kulturtheoretische Terminologie wird in der dichten Beschreibung eher heuristisch eingesetzt, sie kann nur zu aufschlussreichen Sichtweisen führen, wenn sie »vermöge einer präzisen Charakterisierung der

126 Gabriele Mante: Spuren lesen. Die Relevanz kriminalistischer Methoden für die archäologische Wissenschaft. In: Ulrich Veit/Tobias L. Kienlin/Christoph Kümmel/ Sascha Schmidt (Hrsg.): Spuren und Botschaften. Interpretationen materieller Kultur. New York/München/Berlin 2003, S. 157-172.

127 Dass diese Konzeption in ihrer Haupttendenz ein Beispiel für das Adaequationsparadigma der Hermeneutik ist, haben wir oben ausgeführt (s. Kap. 12). Doch tatsächlich findet sich dieses Paradigma bei Geertz verknüpft mit stark deskriptiven Elementen.

128 Clifford Geertz: Dichte Beschreibung. Beiträge zum Verstehen kultureller Systeme, Frankfurt a.M. 1991 (im Orig. 1973), S. 35. 
Tatsachen $\aleph^{129}$ auf die Beschreibungsebene bezogen bleibt. Wegen der Fülle der Details sind genaue sprachliche Formulierungen und nüchterne Darstellungsformen charakteristisch: »Die Beschreibung liebt die lexikalische Liste, die Nomenklatur, sie akkumuliert und summiert«, bemerkt Klaus Scherpe, der das Genre des Beschreibens als literarische Form untersucht. ${ }^{130}$

Solche Formen dienen zugleich der Gegenstandssicherung: in Formaten wie Listen, Karten, Chroniken und Datenbanken werden Beobachtungen aufgezeichnet und auf Dauer gestellt. Werfen wir mit dem Wissenschaftstheoretiker und Molekularbiologen Hans-Jörg Rheinberger einen Blick auf zwei Manifestationsformen solcher Praktiken: Herbarien und Präparate.

In einigen wissenschaftlichen Disziplinen besteht die Arbeit zu einem großen Teil in der Präparierung von Erkenntnisgegenständen, ja: der Sinn der Wissenschaft besteht hier geradezu darin, Gegenstände auf solche Weisen $\mathrm{zu}$ fixieren und zu bestimmen. Was hier - im Unterschied zu den Auseinandersetzungen um Empirismus und Positivismus - außer Frage steht ist, dass es sich bei diesen Gegenständen nicht um unmittelbare, rohe Fakten handelt. Viel zu groß ist die Anstrengung, ihre Beschaffenheiten dem Lauf der Zeit zu entreißen, als dass hier übersehen werden kann, dass ihre jeweilige Erscheinungsweise durch die Wissenschaften erst hervorgebracht ist. Rheinberger verweist auf den französischen Wissenschaftsphilosophen Gaston Bachelard und schreibt, dieser habe

»eindringlich darauf hingewiesen, dass die modernen Naturwissenschaften [für welche ihm in den zwanziger und dreißiger Jahren des vorigen Jahrhunderts die zeitgenössische Mikrophysik Pate stand] eine ganze >Phänomenotechnik, eine Technik der Erscheinungen - man müsste vielleicht korrekter sagen, eine Technik des In-Erscheinung-Bringens - in Szene setzen. « ${ }^{131}$

Es sind bestimmte Wissenschaften, in denen sich diese Vorgänge am deutlichsten zeigen:

129 Geertz, 1991, S. 40.

130 Klaus R. Scherpe: Beschreiben, Nicht Erzählen! Beispiele zu einer ästhetischen Opposition. In: Zeitschrift Für Germanistik, 2(1996), S. 368-383, hier S. 370.

131 Hans-Jörg Rheinberger: Epistemologica: Präparate. In: Anke von Heesen/Petra Lutz (Hrsg.): Dingwelten. Das Museum als Erkenntnisort., Schriften des deutschen HygieneMuseums Dresden, Köln/Weimar/Wien 2005, S. 65-76, hier S. 66. 
»Die vorwiegend deskriptiven, systematisierenden Wissenschaften lösen in der Regel ihre Gegenstände aus ihrem angestammten Naturzusammenhang heraus und stellen sie in einen anderen hinein. Ein botanischer Garten, eine Gesteinssammlung oder ein Herbarium sind Beispiele für einen solchen Vorgang. ${ }^{132}$

Auch eine Schlüsseltechnologie der modernen Naturwissenschaft, das Mikroskopieren, erfordert eine solche Präparierung der Erkenntnisgegenstände. Durch Verfestigung, Haltbarmachung, Fixierung und Färbung wird das Untersuchungsmaterial überhaupt erst untersuchbar - ein Vorgang, bei dem, wie Rheinberger anmerkt, »die Unterscheidung zwischen Fakt und Artefakt zur epistemologischen Schlüsselfrage wird «. ${ }^{133}$ Auch die Streifenmuster, die die molekularbiologische Elektrophorese bildet, sind chromatographische Präparate, Aufzeichnungen der DNA, die im Material selbst erzeugt werden.

Alle Erkenntnisgegenstände dieser Art unterscheiden sich kategorial von wissenschaftlichen Modellen.

»Das Modell hält sich gleichsam in einem anderen Medium auf, es ist geradezu definiert durch den Übergang vom Gegenstand, den es modelliert, in ein anderes Medium.«Im Unterschied dazu »hat das Präparat gewißermaßen teil an der Materialität des untersuchten Sachverhalts. Es ist, wenn man so will, aus dem gleichen Stoff gemacht wie dieser. Es ist eine Figuration, die diesem Stoff abgerungen ist. $^{134}$

Legt man diesen Gedanken zugrunde, ist leicht zu erkennen, dass auch in anderen Disziplinen präparierte Gegebenheiten eine zentrale Rolle spielen. Der gesicherte archäologische Fund, der Bestand der Quellen in den Geschichtswissenschaften, das Interviewmaterial in der Soziologie, die Protokolle von Beobachtungen und Messungen - all die Formen, in denen solche Daten aufgezeichnet und dargestellt sind. Radikale Anhänger des Deskriptivismus wünschen sich, es würde bei Wissenschaft stets bei solchen Aufzeichnungen bleiben.

\footnotetext{
132 Rheinberger, 2005, S. 65.

133 Rheinberger, 2005, S. 71.

134 Rheinberger, 2005, S. 67.
} 


\section{Wissenschaft und die Ordnung der Dinge: Relationen, Strukturen und die Praktiken des Vergleichens und Typisierens (der taxonomische Ansatz)}

Während im deskriptiven Bild von Wissenschaft die Identifikation, Erfassung und Charakterisierung von Gegenständen im Zentrum steht, verschiebt sich der Blickwinkel im taxonomischen Ansatz der Naturalis Historia auf die Beziehungen zwischen den Dingen und den Ordnungen, die sie bilden. Während im deskriptiven Ansatz die Dinge als einzelne Vorkommnisse, lediglich in ihren Eigenschaften und Verortungen in Raum und Zeit zum Gegenstand werden, geht der taxonomische (von gr. taxis: Ordnung) davon aus, dass die wissenschaftlich erschließbare Welt in sich geordnet ist. Die Frage ist hier, wie die Welt geordnet ist, welche Beziehungen bestehen, welche Unterschiede und welche Gemeinsamkeiten zwischen den Dingen festzustellen sind. Begriffe wie Art, Gattung, Typus, Kriterium und Verwandtschaft bilden die Grundlage dieses Bildes von Wissenschaft und Praktiken wie vergleichen, einordnen und klassifizieren prägen es.

Neben den traditionell naturgeschichtlichen Fächern wie Botanik, Zoologie und Mineralogie entstanden in diesem Paradigma eine Reihe anderer Fächer wie vergleichende Geschichtswissenschaften, vergleichende Kunstgeschichte, vergleichende Rechtswissenschaft, vergleichende Politikwissenschaft, International vergleichende Soziologie und ein Zweig der Linguistik: die Sprachtypologie. Auch viele andere Klassifikationen wie die der Fundstücke der Archäologie oder der Systematik der Bakteriologie stehen in diesem Horizont.

Doch auch dieses Bild von Wissenschaft ist von einigen verwirrenden Bruchlinien durchzogen. Auch hier sind es insbesondere zwei geistesgeschichtliche Strömungen, die mit taxonomischem Denken assoziiert sind, im Grunde aber auf andere Wissenschaftsparadigmen hindeuten. Zum einen ist dies der Ansatz einer Universal-generativen Grammatik, zum anderen die wirkmächtige Strömung des Strukturalismus. Sehen wir uns diese beiden tendenziell anders gearteten Verwandten des taxonomischen Denkens an:

Eine Grundfrage der Sprachwissenschaften: Gibt es unbegrenzt viele unterschiedliche Arten von Sprachen oder handelt es sich um Varianten, die letztlich auf nur eine einzige Sprachform zurückzuführen sind?

Die Suche nach Grundformen aller Sprachen, nach Sprachuniversalien, wurde im 20. Jahrhundert angetrieben durch die Arbeiten des US-amerika- 
nischen Linguisten Noam Chomsky, dessen Transformationsgrammatik auf der Annahme beruht, dem Spracherwerb liege ein "genetisch determiniertes Prinzipiengerüst ${ }^{135}$ zugrunde, dessen Möglichkeiten in verschiedenen Sprachen auf verschiedene Weisen ausgeprägt werden. Der Sprachenvergleich dient hier dazu, hinter den Oberflächenstrukturen der sprachlichen Varianten das Regelgerüst freizulegen, das als Tiefenstruktur ihre Erzeugung bestimmt (Chomsky spricht hier von generativer Syntax).

Mit der Annahme eines »Spracherwerbsmechanismus« zeigt sich aber ganz deutlich, dass wir uns hier nicht im Spektrum der naturgeschichtlichen Ansätze, sondern in demjenigen des Cartesianisch-Galileischen Wissenschaftsbildes bewegen. Während es der taxonomischen Wissenschaftsauffassung darum geht, eine Übersicht über die Gegenstände innerhalb ihrer eigenen Ordnung zu gewinnen, sucht die Cartesianisch-Galileische Wissenschaftsauffassung nach einer Ordnung hinter den Dingen, nach einem Regelsystem, das diese bestimmt und in ihren Grundformen determiniert, wenngleich sie nie unmittelbar, sondern nur in den Erscheinungen hervortritt.

Kommen wir zurück zum taxonomischen Wissenschaftstypus. Die Dominanz des Bildes, das Wissenschaft mit der Suche nach im Verborgenen wirkenden Regelsystemen identifiziert, führte innerhalb des taxonomischen Denkens zu tiefreichenden Verunsicherungen. Sie zeigen sich darin, dass die Operation Vergleichen ${ }^{136}$ (und mit ihr das Ordnen und Klassifizieren) sehr unterschiedlich bewertet wird. Während sie den einen als grundständiges und auch kritisches Verfahren der Wissenschaften gilt, durch das neue Phänomene in Abgrenzung und Beziehung zu bekannten Phänomenen erschlossen werden, gilt sie den anderen als unkritische Angleichung und Rubrizierung von Gegenständen in vorgefertigte Schubladen. Wird das >Schubladensystem ‘ als universale Ordnung verstanden, die allen Phänomenen in einem Bereich zugrunde liegt, so reizt dies zum Widerspruch: werden hier überhaupt Anstrengungen unternommen, dem Gegenstand in seiner Eigenheit gerecht zu werden? Und wird hier nicht aus dem Blick ver-

135 Hadumod Bussman: Lexikon der Sprachwissenschaften. Eintrag >Generative Syntax [Auch: Cenerative Grammatik, (Cenerative) Transformationsgrammatik], Stuttgart 2008, S. 222.

136 So der Titel eines viel beachteten Aufsatzes von Joachim Matthes (1992): The Operation Called `Vergleichen<. In: ders.: Zwischen den Kulturen? Die Sozialwissenschaften vor dem Problem des Kulturvergleichs, (Soziale Welt Sonderband 8), Göttingen 1992, S. 75-102. 


\section{BASISWISSEN 11: Erscheinung und Wirklichkeit - die Suche nach einer verborgenen Ordnung}

Die Idee, dass es hinter den Dingen eine eigene Ordnung gibt, die diese Dinge und ihr Verhalten hervorbringt, diese Idee spielt eine entscheidende, aber kaum beachtete Rolle in der Wissenschaftstheorie. Was ist das für eine Idee?

Eine anschauliche Darstellung findet sich in Martin Hollis Kapitel Die Vernunft aufder Suche nach einer verborgenen Ordnung: Hollis zitiert den Cartesianer Bernard de Fontanelle (1657-1757) mit den Worten, »daß die Natur einem Opernhaus ganz ähnlich ist, in dem man von seinem Standort die Bühne nicht so sieht, wie sie wirklich ist. Bei deren Anlage werden Vorteile ausgenutzt, so daß das ganze Räderwerk und die Bewegungen unsichtbar bleiben«. (Hollis 1995, 45)

Diese Konzeption hatte eine mächtige Vorläuferin: Platons Weltbild, dem gemäß hinter den Phänomenen eine nur näherungsweise erkennbare Ordnung der Ideen steht, die vollkommen ist und an der die Erscheinungen nur teilhaben (methexis). Betrachtet man, wie in der Neuzeit geschehen, diese Ideenordnung als Ordnung mathematischer Formen und interpretiert Mathematik wiederum im Geiste der Beweislehre als mechanische Folgerungstechnik, so kommt es zum Bild des Opernhauses: die ideale, wahre Ordnung wird jetzt zum Mechanismus, der die Erscheinungen allererst hervorbringt.

Von da aus schreibt sich die Idee eines generativen Mechanismus in alle die Wissenschaften, in denen nach zugrundeliegenden universalen Gesetzmäßigkeiten für gegebene Erscheinungen gesucht wird (so z.B. in die generative Grammatik der Sprachwissenschaften, in die evolutionäre Kulturtheorie und auch in der Annahme, dass sich menschliches Ver-

loren, dass jede Zuordnung zu Typen, Klassen, Arten, Epochen, Kulturen ein erkenntnistheoretisch prekärer Akt ist? Die Verunsicherung durch solche Fragen hat sich tief in die empirischen Geschichts- und Kulturwissenschaften eingegraben. Sie führt dort zu so defensiven Wendungen wie etwa, dass »es sich bei Typen um Abstraktionen bzw. mentale Konstrukte, nicht aber 
halten oder politische Prozesse auf einen historischen Mechanismus von Klassenzugehörigkeit zurückführen lässt). Am wirkmächtigsten blieb aber stets die Galileische Deutung dieser Hintergrundslogik: es ist die Mathematik, in der das wahre Buch der Natur geschrieben ist, und Wissenschaft zu treiben bedeutet, herauszufinden, wie die mathematische Struktur der Welt die Erscheinungen hervorbringt.

Schon Aristoteles hatte die Platonische Konzeption mit dem Hinweis auf die darin eingeschriebene Verdopplung der Welt mitsamt einer Kluft (chorismos) zwischen Ideen und Erscheinungen kritisiert und es war gerade diese Konzeption, gegen die sich die deskriptive Wissenschaftsauffassung stets gewandt hatte. Für die Beschreibungslehren ist es reine Metaphysik, hinter dem positiv Gegebenen und seiner Ordnung eine zweite, verborgene, >wahre Ordnung zu postulieren - eine doppelte Ontologie.

$\mathrm{Zu}$ unterscheiden ist diese doppelte Ontologie vom erkenntnistheoretischen Dualismus, bei dem es um den Gegensatz von Subjekt und Objekt, von Geist und Welt, kurz: um die Frage geht, wie die Dinge zu Wissen werden; wie sich Wirklichkeit im menschlichen Geist oder der Sprache abbildet. Während die doppelte Ontologie das Sein selbst verdoppelt (in eine Seite der Erscheinungen und in eine der dahinterliegenden Ordnung), problematisiert der erkenntnistheoretische Dualismus das Verhältnis von Wirklichkeit und ihrer Auffassung - auch eine Art Verdopplung, doch in dieser Verdopplung gibt es keine wahre Ordnung hinter den Dingen, sondern eine problematische Ordnung der Repräsentation der Dinge (in Geist, Subjekt, Sprache). Mehr dazu bei BASISWISSEN 13: Konstruktivismus.

Martin Hollis: Soziales Handeln. Eine Einführung in die Philosophie der Sozialwissenschaften, Berlin 1995.

um reale Objekte oder historische Entitäten handelt« (so zu lesen in einer Einführung zur Prähistorischen Archäologie). ${ }^{137}$

137 Manfred K.H. Eggert: Prähistorische Archäologie. Konzepte und Methoden, Tübingen/Basel 2001, S. 139. 


\section{VERTIEFEND Probleme im Vergleichen}

Die Lage verkompliziert sich dadurch, dass neben den drei genannten eine vierte Deutungsoption ins Spiel kommt: Denn fragt man danach, worauf Gemeinsamkeiten und Unterschiede beruhen, die ein Vergleich zu Tage fördert, gibt es nicht nur die drei Antworten, die wir bisher in den Blick genommen haben. Bisher haben wir neben der (1.) eigentümlich taxonomischen Antwort, dass sich in ihr die Ordnung der Dinge, wie sie nun einmal gegeben ist, zur Darstellung bringt, (2.) die (von diesem Standpunkt betrachtet) extrem defensive Auffassung kennengelernt, dass solche Klassifikationen mentale (oder soziale) Konstrukte sind und die dritte, dass sie generiert ist aus einem verborgenen, universalen Regelwerk der Natur (3.). Nun gibt es aber noch eine vierte Variante: zwei oder mehr Gegenstände können Gemeinsamkeiten aufweisen nicht, weil sie schlicht einander ähnlich sind und auch nicht, weil wir sie als ähnlich ansehen oder weil ein quasi-naturgesetzlicher Mechanismus zugrunde liegt, sondern weil sie in realer Beziehung stehen. Überraschende Gemeinsamkeiten z.B. zwischen Kulturen, Sprachen oder Lebewesen können auch dadurch zustande kommen, dass die eine von der anderen oder beide wechselseitig charakteristische Züge übernommen haben. Adaption und Entlehnung sind Vokabeln, die diese Variante der Auffassung von Gemeinsamkeit ausdrücken.

Es ist dieser Einfluss des einen auf das andere, der in der Suche nach Universalien gerade ausgeschlossen werden soll. Hierher gehört das Stichwort Galton's problem - das eigentlich nicht Galtons Problem war, sondern das des von ihm kritisierten Edward Tylor, der glaubte, aus Daten zu Heiratsinstitutionen aus 350 Kulturen einen evolutionären Trend von maternalen zu paternalen Abstammungslinien ablesen zu können. ${ }^{138}$ Galton hatte eingewandt, dass dieser ,Trend ebenso gut aus gemeinsamen Ursprüngen der Kulturen oder Entlehnungen zwischen den Kulturen zurückgeführt werden könnte - also auf ein historisches Faktum und nicht auf eine verborgene universale Regularität hinweist.

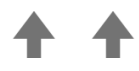

138 Einen guten Überblick gibt Wikipedia im Eintrag: Galton's problem. In: Wikipedia, The Free Encyclopedia, geprüft 12. August 2020. Zur Problematik allgemein: Hartmut Kälble/ Jürgen Schriewer (Hrsg.): Vergleich und Transfer. Komparatistikin den Sozial-, Geschichts- und Kulturwissenschaften, Frankfurt a.M. u.a. 2003. 
Verbunden mit dieser Verunsicherung ist die Verwicklung des taxonomischen Denkens mit einem weiteren geistesgeschichtlichen Paradigma, das die taxonomische Ordnung der Dinge als Ordnungen des Denkens interpretierte und letztlich ins Wissenschaftsparadigma der Kritik führte: der Strukturalismus.

Strukturalismus bezeichnet ein wissenschaftliches und intellektuelles Paradigma, das im 20. Jahrhundert ausgehend von den Sprachwissenschaften auch in der Ethnologie, in der Psychologie, Literaturwissenschaft und Philosophie wirksam wurde. Der Strukturalismus ist ein Spross der naturgeschichtlichen Wissenschaftsauffassung, insofern er Beziehungssysteme zum Gegenstand hat. Allerdings radikalisiert er dieses Bild in einer ganz bestimmten Weise: Es geht hier nicht mehr um Ordnungen, die durch die Eigenschaften der Dinge gebildet werden, sondern um Ordnungen, die die Dinge in ihren wesentlichen Eigenschaften erst hervorbringen und bestimmen. Die Relation wird primär, das Relationierte in Abhängigkeit davon bestimmt.

\section{VERTIEFEND Strukturalismus und die Ordnung der Differenzen}

$\mathrm{Zu}$ beobachten ist dieser Übergang bereits im Ursprung des modernen Strukturalismus, in Ferdinand de Saussures Cours de linguistique générale, ein Werk, das aus Mitschriften dreier Vorlesungen zwischen 1906 und 1911 hervorging. Saussure erläutert dort seine Auffassung, dass Sprache kein Gebilde von in sich positiv bestimmten Elementen, sondern ein System von Relationen sei, so: WWie sich die Laute einer Sprache in ihrer Unterscheidbarkeit $\mathrm{zu}$ anderen Lauten bestimmen, so auch die Bedeutungswerte einer Sprache. Das Wort sheep im Englischen habe einen anderen sprachlichen Wert als das Wort mouton im Französischen, obwohl beide sich auf das gleiche Tier beziehen. Da für Schaf als Speise im Unterschied zum Französischen im Englischen noch ein weiteres Wort existiert, nämlich mutton, verändert sich auch die Funktion des Wortes sheep. Der Inhalt eines Wortes sei deshalb erst »richtig bestimmt nur durch Mitwirkung dessen, was außerhalb seiner vorhanden ist. ${ }^{139}$

139 Ferdinand de Saussure: Grundlagen der allgemeinen Sprachwissenschaft, Berlin 1967 (1906-1911), S. 138. 
So kommt Saussure zu der Auffassung,

»daß es in der Sprache nur Verschiedenheiten gibt. Mehr noch: eine Verschiedenheit setzt im allgemeinen positive Einzelglieder voraus, zwischen denen sie besteht.; in der Sprache aber gibt es nur Verschiedenheiten, ohne positive Einzelglieder. $\ll^{140}$

Der Gedanke, dass die »Sprache ein System ist, dessen Glieder sich alle gegenseitig bedingen «, ${ }^{141}$ hatte vielfältige und weitreichende Wirkungen für die taxonomische Wissenschaftsauffassung im 20. Jahrhundert. Die Ordnung der Dinge erschien nun immer weniger als die der einen kosmologischen Ordnung. ${ }^{142}$ Vielmehr lag es nun nahe anzunehmen, dass es nicht nur unterschiedliche Typen von Sprache, sondern zugleich unterschiedliche Ideensysteme ${ }^{143}$, unterschiedliche Weisen der Welterzeugung ${ }^{144}$ gibt, die in sich als differentielle Systeme bestimmt sind. Die Taxonomien, die sie ausbilden, bilden nicht mehr die Welt oder bestimmte Weltausschnitte $a b$, sondern zeigen sich nun daraufhin geformt - so drückt es der strukturalistische Anthropologe Claude LéviStrauss aus -, dass sie "gut zu denken sind $« .{ }^{145}$ So führte gerade eine Renaissance des Taxonomischen zu dessen fundamentalster Umdeutung: aus der Erforschung der Ordnung der Welt wurde die Erforschung der Ordnungen des Denkens, das die Ordnung der Welt zum Gegenstand hat. Taxonomisches Denken wurde so zu einem Nachdenken und Forschen über unterschiedliche Formen und Möglichkeiten des Denkens und Ordnens. Ein Streit um die Universalität und Relativität kultureller Strukturen brandete auf - Stichwort

140 Saussure, 1967, S. 143.

141 Saussure, 1967, S. 136.

142 Ordnung der Dinge ist der deutschsprachige Titel von Michel Foucaults einschlägiger Monographie Les mots et les choses, Paris 1966.

143 Peter Winch hat Wittgensteins Sprachspieldenken in eine Konzeption von Ideensystemen transformiert (The Idea of a Social Science, London 1958). Vgl. dazu: Werner Kogge: Die Grenzen des Verstehens. Kultur - Differenz - Diskretion, Weilerswist 2002, S. $102-109$.

144 Weisen der Welterzeugung ist der deutschsprachige Titel von Nelson Coodmans Buch Ways of Worldmaking, Indianapolis 1978.

145 Claude Lévi-Strauss: Das Ende des Totemismus, Frankfurt a.M., 1965, S. 116. Vgl. zur Intelligibilität klassifikatorischer Ordnungen auch Claude Lévi-Strauss: Das wilde Denken, Frankfurt a.M. 1973. 
Rationality-Debatte ${ }^{146}$ - und die Frage nach einer zeitgemäßen Ordnung des Seienden (griechisch: Ontologie), die nicht mehr von wesenhaften Eigenschaften der Dinge ausgeht (Substantialismus) beschäftigte seitdem die Gemüter.

Die Problemstellung des Strukturalismus erreichte allerdings die in ihrer Praxis tatsächlich vergleichend und ordnend arbeitenden Wissenschaften nur als punktuelle Verunsicherungen in der Weise, wie sie sich in der oben zitierten Einführung in die prähistorische Archäologie niederschlug. Man folgt der Einsicht, dass Typisieren und Ordnen keine unproblematischen wissenschaftlichen Aktivitäten sind, die sich aus den Eigenschaften der Dinge selbst ergeben, aber es fällt schwer, eine Abgrenzung zu dem am besonderen Gegenstand der Sprache gewonnenen Bild von Ordnung allererst generierender Ordnungen zu gewinnen. Das Wort Konstrukt, das sich in Folge des strukturalistischen und poststrukturalistischen Denkens Bahn brach, wird hier zur Selbstversicherungsformel wissenschaftlicher Reflexivität. Das Thema Konstruktivismus behandeln wir unten nochmal ausführlicher (siehe BASISWISSEN 13: Konstruktivismus - ein Schlagwort, unterschiedliche Bedeutungen).

Hier ist festzuhalten: die Verwirrungen um das Mega-Thema Konstruktivismus sorgten dafür, dass die Frage, wie ein kritisches Herangehen im Typisieren und Ordnen aussieht, häufig in den Hintergrund geriet. Löst man aber das taxonomische Denken von den großen Fragen nach der Beschaffenheit der Welt und der Einheit oder Vielfalt wahrer Weltauffassungen, so kommt ein methodologischer Kern zum Vorschein, der für unsere Fragestellung nach unterschiedlichen Wissenschaftstypen bedeutsam ist:

Schon Aristoteles hatte methodologische Fragen des Ordnens in Taxonomien angeschnitten, etwa in seiner Schrift Über die Teile der Lebewesen, auch Carl von Linné stellt seiner Systema Naturae methodische Überlegungen zu Begründetheit der taxonomischen Begriffe Klasse, Ordnung, Gattung und Art voran. ${ }^{147}$

146 Vgl. dazu Sigrid Frethlöh: Relativismus vs. Universalismus. Zur Kontroverse über Verstehen und Übersetzen in der angelsächsischen Sprachphilosophie: Winch, Wittgenstein, Quine, Aachen1989; Werner Kogge: Die Grenzen des Verstehens. Kultur - Differenz - Diskretion, Weilerswist 2002, Kap.: II.3.2 >Die Debatte um die Rationalität der Kulturen<, S. 109-117.

$147 \mathrm{Vgl}$. zu diesem Textteil in der komplexen Editionsgeschichte von Linnés Systema Naturae: Eintrag »Methodus«. In: Wikipedia, Die freie Enzyklopädie. Bearbeitungsstand: 
Einige für die vergleichenden und klassifizierenden Wissenschaften entscheidende Punkte hat der Historiker Marc Bloch in seinem Text Für eine vergleichende Geschichte der europäischen Gesellschaften (sowie einem Lexikonartikel zum Stichwort Vergleich) ${ }^{148}$ herausgestellt: Einer davon betrifft die Frage der Vergleichseinheiten. In den historischen Wissenschaften, so Bloch, werden häufig unreflektiert politische Einheiten vorausgesetzt, sodass etwa deutsche und französische Städte des Mittelalters miteinander verglichen werden, ohne dass beachtet wird, dass einige dieser Städte in ihrer Einheit von Bistum und Handelsstadt über Grenzen hinweg viel stärkere Gemeinsamkeiten aufweisen als mit anderen Städten der vorausgesetzten politischen Einheit. Dieses Beispiel zeigt, dass das Vergleichen und Klassifizieren nicht einfach als ein Sortieren in vorgefertigte Schubladen hinein betrachtet werden darf, vielmehr als eine Suche nach angemessenen Vergleichseinheiten, die auf Kenntnis und Klugheit beruht. Ein zweiter Punkt Blochs ist, dass das Vergleichen nicht mit einer naiven Suche nach Ähnlichkeiten, die sich allenthalben finden lassen, identifiziert werden darf. Ebenso wichtig wie Gemeinsamkeiten sind im Vergleichen Unterschiede. Gerade da, wo unkritisch »scheinbare Ähnlichkeiten ${ }^{149}$ - »Wieviele Unterschiede verbergen sich nicht allein hinter dem Wort `Adel!! s, schreibt Bloch - angenommen werden, kann der Vergleich Einheiten sprengen und lehren, neue Zusammenhänge zu sehen. Fern davon, das taxonomische Denken als naiv-substantialistisches Weltbild abtun zu können, halten wir fest, dass auch in ihm ein eigenes forscherisches Potential liegt; die Entstehung und Blüte einer Vielzahl komparatistischer Disziplinen lässt sich auch so verstehen.

18. Mai 2014.

148 Beide Texte finden sich abgedruckt in: Marc Bloch: Aus der Werkstatt des Historikers. Zur Theorie und Praxis der Geschichtswissenschaften, Frankfurt a.M. u.a. 2000. >Vergleich (im Orig. 1930); S. 113-121; >Für eine vergleichende Geschichte der europäischen Gesellschaften< (im Orig. 1928), S. 122-159.

149 Bloch, 2000, S. 121. 


\section{Die Rolle der Statistik: Daten, Mengen, Regelmäßigkeiten - und eine Irrfahrt ins Reich der Wahrscheinlichkeit}

Wenden wir uns nun der Statistik zu. Was ist Statistik? Vor allem: Haben wir es hier mit einer neuen Variante von Wissenschaft zu tun? Ist Statistik mehr als das, was wir vom taxonomischen Ansatz schon kennen: Daten nach Kategorien unterteilen und in Rubriken einordnen; typisieren und klassifizieren?

Ein Unterschied fällt ins Auge: beim Erstellen von Statistiken wird gezählt. Quantitative Verhältnisse und Prozentsätze erscheinen geradezu als Wesensmerkmal des Statistischen. Und dabei bleibt es nicht: Statistik ist heute nicht nur der Name für quantitative Datenerhebungen, sondern auch für eine mathematische Disziplin. Diese Mathematik ist es, die in vielen Statistik-Kursen, z.B. in der Psychologie, der Soziologie oder der Epidemiologie gelehrt und gelernt wird. Haben wir es also mit einer Anwendung mathematischer Verfahren auf Gegenstandsbereiche zu tun, ähnlich wie in der Physik? Oder ist der Einsatz solcher Verfahren eher eine Ausweitung der Methoden im sammelnden und ordnenden Wissenschaftsparadigma der Naturalis historiae?

Blicken wir auf gegenwärtige Debatten in diesem Feld, so fällt zweierlei auf: Erstens, dass das Erheben, Speichern und Verarbeiten von Daten mit steiler Konjunktur zugenommen hat; es sieht aus, als wäre statistisches Wissen auf ungebremstem Vormarsch. Zweitens aber wird Kritik an Standardverfahren der Statistik immer deutlicher vernehmbar: »Vorsicht Statistik! «150 heißt ein aktueller Titel eines Wissenschaftsmagazins, Das Einmaleins der Skepsis ein Buchtitel ${ }^{151}$, Significance Tests as Socery ein wissenschaftlicher Artikel. ${ }^{152}$ Vom »Fluch des P-Werts « liest man, von »statistischen Ritualen«, "mindless statistics« und "null science $« .{ }^{153}$ Ein Resumee zu einer breit angelegten Studie zu Statistik im Forschungsprozess lautet:

150 Spektrum der Wissenschaft. Physik, Mathematik, Technik, Spezial: Vorsicht Statistik! Vom Gesetz der großen Zahlen bis zu Klimarekorden, Heft 3(2017).

151 Gerd Gigerenzer: Das Einmaleins der Skepsis. Über den richtigen Umgang mit Zahlen und Risiken, München/Berlin 2015.

152 Charles Lambdin: >Significance tests as sorcery: Science is empirical - significance tests are not. In: Theory \& Psychology 22, 1 (2012), S. 67-90.

153 Uwe Saint-Mont: Statistik im Forschungsprozess. Eine Philosophie der Statistik als Baustein einer integrativen Wissenschaftstheorie, Heidelberg u.a. 2011, S. 93. 
»Im großen und ganzen stehen Naturwissenschaftler der >offiziellen Statistik« recht reserviert gegenüber [...] In den Sozialwissenschaften inklusive der Medizin wurden jedoch im Fahrwasser des Erfolgs quantitativer Methoden auch Hypothesentests und einige andere statistische Verfahren zum de facto Standard. Es sollte jedoch nicht verwundern, dass die Einsichten, welche man mithilfe der [...] >kochbuchartigen Schulstatistikı gewonnen hat, eher dürftig sind. [Man] verwendet [...] zwar fleißig ihre Begriffe und berechnet darüber hinaus emsig $p$-Werte und andere Statistiken, betrachtet dieses jedoch nicht ganz zu unrecht als lästige Pflichtübung. Statistik nach strengen Regeln betrieben hört sich wissenschaftlich an, beeindruckt mit exakten Zahlen, schwer verständlichen Argumenten und macht Arbeiten vor allem publikationsfähig. Primär aus diesen Gründen wird sie wohl von vielen Wissenschaftlern benutzt, nicht weil man ihren intrinsischen Forschungswert schätzte. ${ }^{154}$

Was ist hier schiefgelaufen? Zumindest ein Teil von Statistik scheint eher die Funktion $\mathrm{zu}$ haben, wissenschaftsförmige Effekte $\mathrm{zu}$ simulieren als wissenschaftliche Erkenntnisse zu produzieren. Und doch ist klar, dass so wichtige wissenschaftliche Vorhaben wie z.B. Klimamodelle, Bevölkerungsprognosen oder medizinische Studien auf Statistiken angewiesen sind. Die Situation - in sich so widersprüchlich - gibt zu vermuten, dass hier ein Fall von Selbstmissverständnis in den Wissenschaften vorliegt, eine Art Kategorienfehler in der Anlage von Studien, der durch unpassende Erwartungen erzeugt wird.

Sehen wir uns die Entstehung der aktuellen Situation an: Zunächst lässt sich feststellen, dass das, was wir heute Statistik nennen, aus der Kreuzung zweier unterschiedlicher Quellen hervorgegangen ist und sich mit der Zeit in mehrere, sehr verschiedenartige Felder ausdifferenziert hat. Die eine dieser beiden Quellen ist die sich in der Neuzeit ausprägende staatswissenschaftliche Statistik, die im Sammeln und Verarbeiten staatsrelevanter Daten besteht: Bevölkerungszahlen, Geburten, Todesfälle, Heiraten, Suizide, Körpergrößen von Rekruten - eine Vielzahl solcher Daten wurden erhoben und mussten dann auch geordnet, ausgewertet und dargestellt werden.

Diese Geschichte der Verwaltungspraktiken durch Daten verband sich nun im 19. Jahrhundert mit einer ganz anderen Entwicklung, nämlich der der Wahrscheinlichkeitstheorie oder Probabilistik. Diese wiederum hatte 
ihre Quelle in der mathematischen Behandlung von Glücksspielen und so nimmt es kaum Wunder, dass das Wort Statistik heute, neben seiner staatspolitischen Bedeutung, auch einen Zweig der Mathematik bezeichnet. ${ }^{155}$

Die gegenwärtige Rolle der Statistik wird aber erst verständlich, wenn wir ein drittes Moment in Rechnung stellen: nämlich das Entstehen einer Wissenschaft vom Sozialen. Zu Beginn des 19. Jahrhunderts formierten sich Anstrengungen, aus dem Wissen von der Gesellschaft eine veritable Gesellschaftswissenschaft zu formen, eine Physique Sociale sollte entstehen - Vorläufer dessen, was sich schließlich als 'Sociologie etablierte. Der Begründer der Physique Sociale war nun aber ein Statistiker. Adolphe Quetelet, zuvor Mathematikdozent und Direktor eines astronomischen Observatoriums (was für das Folgende nicht unwesentlich ist), bekleidete seit 1841 das Amt des Präsidenten der statistischen Zentralkommission Belgiens. Sehen wir uns an, wie Quetelet das Neuartige seines wissenschaftlichen Ansatzes darstellt:

Er fragt: „Erfolgen die moralischen und intellektuellen Handlungen des Menschen nach bestimmten Gesetzen? ${ }^{156}$ Und gibt als methodische Leitlinie an:

»Vor allem müssen wir vom einzelnen Menschen abstrahieren und dürfen ihn nurmehr als einen Bruchteil der ganzen Gattung betrachten. Indem wir ihn seiner Individualität entkleiden, beseitigen wir alles, was nur zufällig ist; die individuellen Besonderheiten, die wenig oder keinen Einfluß auf die Masse haben, verschwinden dann von selbst und lassen uns zu allgemeinen Ergebnissen gelangen. $\ll^{157}$

An dieser Stelle nun bietet Quetelet eine Analogie dar, die recht deutlich macht, welche Art von Wissen dieser Typ von Statistik hervorbringt. Er schreibt:

155 Zur Begriffsgeschichte und Diskursgeschichte vgl. Eintrag sStatistik in: Historisches Wörterbuch der Philosophie, Bd. 10, S. 39750-39768 (v. Petra Cehring).

156 Adolphe Quételet: Soziale Physik oder Abhandlung über die Entwicklung der Fähigkeiten des Menschen; n. d. Ausg. letzter Hand (1869) [im Orig. 1835] übers. v. Valentine Dorn u. eingel. v. Heinrich Waentig, 1. Bd., Jena 1914, S. 103.

157 Quételet, 1914, S. $103 f$. 
»So würde, um die Art unseres Verfahrens durch ein Beispiel sinnfällig zu machen, derjenige, welcher einen kleinen Abschnitt einer auf einer Fläche gezogenen sehr großen Kreislinie zu nahe prüfen würde, in diesem Bruchteil nichts weiter sehen, als eine bestimmte Menge materieller Punkte, die mehr oder weniger bizarr, mehr oder weniger willkürlich, mag die Linie im übrigen auch noch so sorgfältig gezogen sein, wie von ungefähr vereinigt sind. Aus größerer Entfernung würde sein Auge eine größere Anzahl Punkte überblicken, die er bereits regelmäßig auf einen Bogen von bestimmter Ausdehnung verteilt sehen würde; noch weiter zurücktretend, würde er bald keinen von ihnen mehr einzeln sehen, aber er würde das Cesetz begreifen, nach dem sie im allgemeinen angeordnet sind, und die Natur der gezogenen Kreislinie erkennen. $\ll^{158}$

Sehen wir uns das Erkenntnisparadigma an, das in dieser Analogie zum Ausdruck kommt: Die regelmäßige Form erscheint erst dann, wenn eine große Zahl von Elementen gleichzeitig in den Blick genommen wird. Dass dies hier durch Entfernung geschieht, ist nur der Analogie geschuldet. Entscheidend ist die große Zahl. Während das einzelne Element sich scheinbar regellos verhält, werden mit Blick auf eine große Zahl gleichartiger Elemente Regelmäßigkeiten sichtbar. In diesen Regelmäßigkeiten sieht Quetelet soziale Gesetze ausgedrückt. Doch um was für einen Begriff von Gesetz handelt es sich hier?

Quetelets erstes Beispiel sind Kriminalstatistiken aus französischen Gerichten. Die frappierend regelmäßige Anzahl der Morde und die gleiche Verteilung der dazu verwendeten Mittel offenbaren, so Quetelet, Gesetzmäßigkeiten der Gesellschaft. Doch wie drückt sich diese Gesetzmäßigkeit aus?

Die Analogie zur Kreislinie aus Punkten kann uns hier auf die richtige Spur bringen: Quetelet sucht weder - in der Tradition Euklids - nach einer Konstruktionsregel für Kreise, noch - in der Tradition Aristoteles' - nach prinzipiellen Bestimmungen von Kreisförmigkeit, vielmehr ist die Kreisform hier selbst das gesuchte Gesetz. Für ihn bedeutet Wissenschaft: in einer unübersichtlichen Mannigfaltigkeit die »Ordnung im Gange der Natur ${ }^{159}$ sichtbar zu machen. Wissenschaftliche Erkenntnis heißt hier also: die Wahrnehmung gestalthafter Ordnung in einer großen Zahl individueller Elemente. Und weil es bei dieser Form von Wissenschaft um Ordnungsgestalten geht, kann es auch nicht verwundern, dass Quetelet die Frage, ob 
Abb. 3: Verteilung von Verbrechensarten.

\begin{tabular}{|c|c|c|c|c|c|c|}
\hline & 1826 & 1827 & 1828 & 1829 & 1830 & 1831 \\
\hline Morde im allgemeinen. & 241 & 234 & 227 & 230 & 205 & 266 \\
\hline mittels Gewehr und Pistole. & 56 & 64 & 60 & 61 & 57 & 88 \\
\hline $\begin{array}{c}\text { Säbel, Degen, Stilett, } \\
\text { Dolch usw. . . . . }\end{array}$ & 15 & 7 & 8 & 6 & 12 & 30 \\
\hline Messer . . . . . . & 39 & 40 & 34 & 46 & 44 & 34 \\
\hline Stock, Rohr usw. & 23 & 28 & 31 & 24 & 12 & 21 \\
\hline $\begin{array}{l}\text { Steine } \\
\text { schneidender, stechender } \\
\text { und zerschmetternder }\end{array}$ & 20 & 20 & 21 & 21 & 11 & 9 \\
\hline Instrumente . . . . & 35 & 40 & 42 & 45 & 46 & 49 \\
\hline $\begin{array}{l}\text { Erdrosselungen } \\
\text { durch in die Tiefe stürzen und }\end{array}$ & 2 & 5 & 2 & 2 & 2 & 4 \\
\hline ertränken . . . . . & 6 & 16 & 6 & 1 & 4 & 3 \\
\hline durch Fuß- und Fauststöße & 28 & 12 & 21 & 25 & 17 & 26 \\
\hline durch Feuer . . . . . . & 28 & 1 & 21 & 1 & 17 & 26 \\
\hline auf unbekannte Art und Weise & 17 & 1 & 2 & 1 & 2 & 2 \\
\hline
\end{tabular}

Quelle: Adolphe Quételet: Soziale Physik, S. 106.

die Statistik »als eine Kunst oder eine Wissenschaft anzusehen ${ }^{160}$ ist, mit Verweis auf die Botanik beantwortet, die sich durch ihre, über das bloße Sammeln und Beschreiben hinausgehende "tiefgründige Klassifikation « ${ }^{161}$ zur Wissenschaft erhob.

Gesetzmäßigkeit als Ordnungsgestalt: unschwer ist zu erkennen, dass wir es hier wieder mit dem Gegensatz zu tun haben, den wir im vorletzten Abschnitt als den zwischen einer beweisenden und einer deskriptiven Physik kennengelernt haben. Für die Deskriptivisten war entscheidend, jegliche nicht phänomenal auftretenden Kräfte als metaphysische Annahme zurückzuweisen. In diesem Sinne ist Quetelet zunächst reiner Deskriptivist. Er schreibt:

"Die Statistik beschäftigt sich mit einem Staate während eines bestimmten Zeitabschnittes; sie sammelt die auf das Leben dieses Staates bezüglichen Elemente, macht sie vergleichbar und stellt sie so zusammen, wie es für die

160 Quételet, 1914, S. 111.

161 Quételet, 1914, S. 112. 


\section{BASISWISSEN 12: Kausalität und Korrelation}

Die Problematik des Unterschiedes von Korrelation und Kausalität tritt beim Übergang von darstellender zu erklärender Statistik auf. Bereits bei Quetelet findet sich der Gedanke, dass durch Vergleich verschiedener Zahlenreihen sich verschiedene Ursachen für Phänomene erschließen lassen müssten. Beobachtet man z.B., dass die mittlere Lebensdauer an unterschiedlichen Orten unterschiedlich ist, so lässt sich danach fragen, welche Faktoren und Einflüsse diese Unterschiedlichkeit hervorbringen. Klima, Wohlstand, Ernährung, Städtebau: Der Vergleich der Daten zur Lebensdauer in Hinblick auf Unterschiede in diesen Faktoren könnte, so der Gedanke, Ursachen der Unterschiede in der Lebensdauer offenbaren. Doch schon Quetelet deutet an, welche Schwierigkeit in diesem Unterfangen liegt: »bis jetzt kennt man nur die Resultate verschiedener Kräfte, welche man nicht nur im einzelnen nicht messen, sondern nicht einmal vollständig aufzählen könnte.« (Quételet, 1914, S. 164)

Die Frage ist aber, ob im genuin statistischen Modell der Erkenntnis ein solcher Begriff von Ursache überhaupt eine Rolle spielen kann. Heinrich Waentig, ein deutscher Nationalökonom, der die Einleitung zu Quetelets Werk von 1914 verfasste, zieht es vor, nicht von Ursachen, sondern von Umständen zu sprechen. In der statistischen Wahrscheinlichkeitsberechnung werde nämlich der Ausdruck Ursache

»ohne irgend eine Bezugnahme auf eine vermutliche Kraft angewendet, die imstande wäre, ein gegebenes Resultat vermöge einer von ihr unzertrennlichen Tätigkeit hervorzubringen. Er bezeichnet nur die Veranlassung für die mehr oder weniger häufige Wiederholung eines Resultates und kann ebensogut in der Entfernung eines Hindernisses wie in einer direkten Handlung bestehen.«(Quételet, 1914, S. 12)

Erkenntnis aller Tatsachen, die sie uns enthüllen können, am vorteilhaftesten ist. ${ }^{162}$

162 Quételet, 1914, S. 112. 
Leider ist das Wort Umstand aus der Mode gekommen und man spricht stattdessen von Korrelationen. Der Unterschied zwischen Korrelation und Kausalität wird gerne an sinnfälligen Beispielen demonstriert, wie dem der statistisch auffälligen Korrelation zwischen Storchenpopulation und Geburtenrate: weniger Störche - weniger Geburten. Wer darin keinen Kausalzusammenhang vermuten möchte, der kann immerhin nach gemeinsamen Umständen suchen - wie etwa Urbanisierung, Industrialisierung etc.

Folgende Abbildung stammt aus einer Sammlung absurder Korrelationen, deren Korrelationskoeffizient hoch ist, ohne dass ein Zusammenhang vermutet werden könnte. Die Entwicklung nicht-kommerzieller Weltraummissionen korrespondiert hier beeindruckend mit der Zahl der Doktorats-Abschlüsse im Fach Soziologie (aus Tyler Vigen: Spurious Correlations. Correlation does not equal causation, New York 2015, S. 61).

Korrelation bezeichnet eine symmetrische, Kausalität eine asymmetrische Beziehung. Die Datenreihen, die miteinander korreliert werden, sind prinzipiell gleichwertig, erst die Deutung der einen als die Ursache für die andere macht daraus eine Kausalbeziehung. Und diese Deutung erfolgt extern, sie kann nicht selbst statistisch begründet werden.

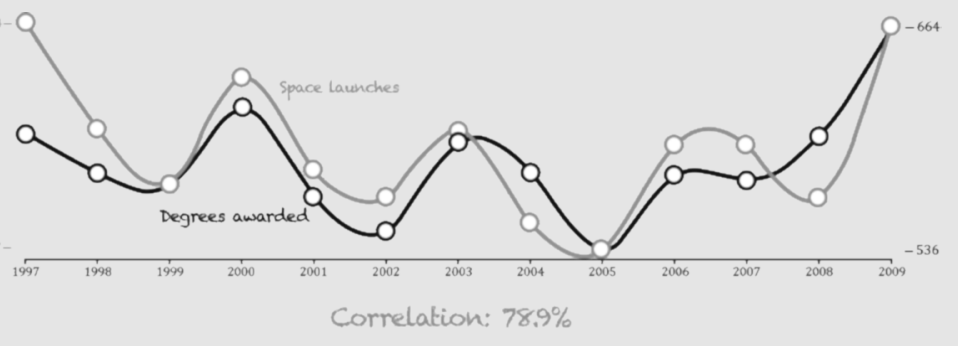

Das entspricht dem Bild, mit dem Quetelet ursprünglich das statistische Erkennen charakterisiert: In den Punktmengen sehen wir Ordnungszusammenhänge, jeder weitere Punkt, jede Hinzufügung von Daten verdeutlicht das Bild eines Zusammenhangs. Die Daten stehen in Beziehung zueinander, sie sind korreliert. In diesem Ordnungszusammenhang allerdings die einen Daten zu Ursachen der anderen zu erklären - dies ist ein bedeutender Schritt. 
Machen wir uns den Übergang von einer deskriptiven zu einer erklärenden Statistik an einem Beispiel klar. Noch einmal Quetelet: »Jeder soziale Zustand setzt also eine gewisse Zahl und eine gewisse Ordnung von Verbrechen voraus, die eine notwendige Folge seiner Organisation sind. ${ }^{163}$ Die Textstelle klingt zirkulär, in der Terminologie von Umständen liegen alle Daten auf der gleichen Ebene: die Häufigkeit von Verbrechen gilt z.B. als ein Umstand für ein prekäres soziales Milieu und ein prekäres soziales Milieu als ein Umstand für die Häufigkeit von Verbrechen. In einem schwachen Sinn kann man auch hier davon sprechen, dass das eine die Ursache des anderen ist. Doch die Beziehung ist symmetrisch und ergibt als Ganzes ein Bild, das sich konzeptuell fassen und durch Daten begründen lässt.

Verwendet man nun aber einen starken Begriff von Ursache, sodass z.B. das soziale Milieu als Ursache für eine Zahl von Verbrechen gedacht wird, so fügt man die Daten in ein asymmetrisches Verhältnis zueinander: die einen bezeichnen die Ursachen, die anderen die Wirkungen. Nun stellt sich eine ganz andere und neue Frage, die im deskriptiv-naturgeschichtlichen Paradigma überhaupt nicht auftaucht, oder aber strikt abgelehnt wird: welche Regel, welcher Mechanismus liegt der Bewirkung des Effekts aus den Ursachen zugrunde?

Das frühe 20. Jahrhundert ist nun die Epoche, in der die neue Sichtweise sich etablierte und mathematisch ausformuliert wurde. Nun geht es nicht mehr um eine Menge von Phänomenen (ob sie nun Punkte am Himmel, Körpergrößen oder Morde waren), sondern um Anzahlen von Experimenten bzw. Beobachtungen und den sich daraus ergebenden Daten.

Symptomatisch für den Übergang ist der neue Gebrauch des Begriffs Population. So liest man:

»Der Begriff Population ist nicht nur auf lebende oder auch nur materielle Dinge beschränkt. Wenn eine Beobachtung, wie beispielsweise eine einfache Messung, unbegrenzt oft wiederholt wird, so ist die Gesamtheit dieser Ergebnisse die Population der Messungen. $\aleph^{164}$

163 Quételet, 1914, S. 107.

164 Ronald A. Fisher/Dora Lucka: Statistische Methoden für die Wissenschaft, Edinburgh [u.a.] 1956, S. 2. 
Die wichtigste Frage, für die Statistik nun zuständig wird, ist, wie viele Beobachtungen notwendig sind, um einen hinreichenden Beleg für eine allgemeine Regel zu erhalten; oder umgekehrt: wie aussagekräftig (»signifikant«) eine bestimmte Datenmenge für eine bestimmte Regelannahme ist. Nullhypothese, P-Wert und Signifikanztest sind die Begriffe, die das Feld der Statistik von nun an beherrschen. Sie haben ihre Quelle in der mathematischen Wahrscheinlichkeitstheorie und finden nun ihre Anwendung in einem bestimmten Typ von >Experiment‘, nämlich dem Prüfexperiment.

Die Geschichte der mathematischen Wahrscheinlichkeitstheorie ist gut erforscht. In einer Reihe von Studien lässt sich nachlesen, wie sich von Pascal, Leibniz und Bernoulli (Jakob) bis zu Laplace und Bayes im 17. und 18. Jahrhundert eine elaborierte mathematische Wahrscheinlichkeitstheorie entwickelte. ${ }^{165}$ Im 19. Jahrhundert nun verbindet sich diese Theorie mit der Praxis der staatlichen Statistik und damit mit der Erhebung und Sammlung großer Mengen empirischer Daten. Von diesem Zeitpunkt an entstand eine Dynamik, in der es immer häufiger so aussah, als ob es gerade Statistik wäre, was Wissenschaft ausmacht - bis nun, in jüngster Zeit, die Rolle von Statistik tiefgreifend fragwürdig wurde.

\section{$\checkmark$ VERTIEFEND Der (Irr-)Weg der Statistik}

Um uns deutlich zu machen, was hier passiert ist und welche Rolle wir der Statistik in den Wissenschaften zuzuschreiben haben, schauen wir uns systematisch einige entscheidende Schritte auf diesem Weg an.

Gehen wir aus von dem einfachsten Fall, den wir kennen gelernt haben: die Kriminalstatistiken Quetelets - wie oben abgebildet. Solche Statistiken fixieren schlichte Daten: Jahr für Jahr wird die Anzahl der Fälle notiert. Übertrüge man die Zahlen in die Form eines Liniendiagramms, so ließe sich eine Linie auftragen, deren nur leichte Schwankungen nach oben und unten die Regelmäßigkeit bezeugt, die Quetelet so imponierte.

Etwas anders verhält es sich schon mit den Körpergrößentabellen, die wir ebenfalls bei Quetelet finden. (Abb. 5) Die Zahlen, die sich in der zwei-

165 G. Gigerenzer/Z. G. Swijtink/T. Porter/L. Daston/J. Beatty/L. Krüger (Hrsg.): Das Reich des Zufalls: Wissen zwischen Wahrscheinlichkeiten, Häufigkeiten und Unschärfen. Heidelberg 1999, S. 19. 
Abb. 5: Größenverteilung von Rekruten.

\begin{tabular}{|c|c|c|c|c|c|c|}
\hline \multicolumn{3}{|c|}{$\begin{array}{c}\text { Größenmaße nach } \\
\text { Metern }\end{array}$} & \multirow{4}{*}{$\begin{array}{c}\text { Zahl der Gezählten } \\
\text { nach dem Größen- } \\
\text { unterschied von } \\
0,0255 \mathrm{~m} \\
\\
4 \\
1\end{array}$} & \multicolumn{2}{|c|}{$\begin{array}{l}\text { Verhältnis der Größe } \\
\text { von } 1000 \text { gemessenen } \\
\text { Eingeschriebenen }\end{array}$} & \multirow[t]{2}{*}{$\begin{array}{c}\text { Unterschied } \\
\text { zwischen den } \\
\text { beobachteten u. } \\
\text { berechneten Werten }\end{array}$} \\
\hline & & & & beobachtet & gemessen & \\
\hline \multicolumn{3}{|c|}{1,397 und darunter } & & \multirow{6}{*}{1} & \multirow{6}{*}{2} & \multirow{6}{*}{-1} \\
\hline 1,422 & " & “ & & & & \\
\hline 1,448 & $"$ & “ & 3 & & & \\
\hline 1,473 & $"$ & “ & 7 & & & \\
\hline 1,499 & $"$ & “ & 6 & & & \\
\hline 1,524 & $"$ & “ & 10 & & & \\
\hline 1,549 & $"$ & “ & 15 & 1 & 3 & -2 \\
\hline 1,575 & $"$ & “ & 50 & 2 & 9 & -7 \\
\hline 1,600 & $"$ & “ & 526 & 20 & 21 & -1 \\
\hline 1,626 & $"$ & “ & 1237 & 48 & 42 & +6 \\
\hline 1,651 & $"$ & “ & 1947 & 75 & 72 & +3 \\
\hline 1,676 & $"$ & $“$ & 3019 & 117 & 107 & +10 \\
\hline 1,702 & $"$ & “ & 3475 & 134 & 137 & -3 \\
\hline 1,727 & $"$ & $"$ & 4054 & 157 & 153 & +4 \\
\hline 1,753 & “ & “ & 3631 & 140 & 146 & -6 \\
\hline 1,778 & $"$ & $“$ & 3133 & 121 & 121 & 0 \\
\hline 1,803 & $"$ & $“$ & 2075 & 80 & 86 & -6 \\
\hline 1,829 & $"$ & $"$ & 1485 & 57 & 53 & +4 \\
\hline 1,854 & $"$ & $“$ & 680 & 26 & 28 & -3 \\
\hline 1,880 & $"$ & $"$ & 343 & 13 & 13 & 0 \\
\hline 1,905 & $"$ & “ & 118 & 5 & 5 & 0 \\
\hline 1,930 & $"$ & $"$ & 42 & 2 & 2 & 0 \\
\hline 1,956 & $"$ & $"$ & 9 & & & \\
\hline 1,981 & " & “ & 6 & 1 & 0 & +1 \\
\hline 2,007 & $"$ & $“$ & 2 & & & \\
\hline \multicolumn{3}{|c|}{ Zusammen } & 25878 & 1000 & 1000 & $\begin{array}{l}-28 \\
-28\end{array}$ \\
\hline
\end{tabular}

Quelle: Quételet: Soziale Physik, S. 145.

ten Kolumne dieser Tabelle finden (die weiteren können wir zunächst außer Acht lassen), sind schon keine Anzahlen von Gegenständen und Ereignissen in der Welt mehr, sondern Zahlen, die sich auf Zahlen beziehen: Man sortiert die gemessenen Körpermaße in Gruppen und schaut sich dann an, wie viele Messungen sich in jeder Gruppe jeweils finden. Das ist die Zahl in der zweiten Spalte. Natürlich lässt sich an dieser Darstellung das empirische Phänomen noch erkennen: wir können uns vorstellen, dass die 25878 Rekruten nach ihrer Körpergröße in Gruppen aufgeteilt und sodann die Gruppen der Größe 
der Gruppe nach aufgestellt würden. Abgesehen von praktischen Schwierigkeiten ist dies denkbar - und doch wird hier schon kenntlich, dass im Interesse nicht mehr an der Größe der Rekruten, sondern an der Größe der Gruppen (gebildet nach Größen der Rekruten) ein spezielles, theoretisches Interesse durchscheint.

Dieses theoretische Interesse sieht nun wiederum sehr schlicht und einfach aus, wenn man diese Daten - wie es Quetelet auch tat - in einer Kurve darstellt. (Abb. 6)

Abb. 6: Gausssche Darstellung.

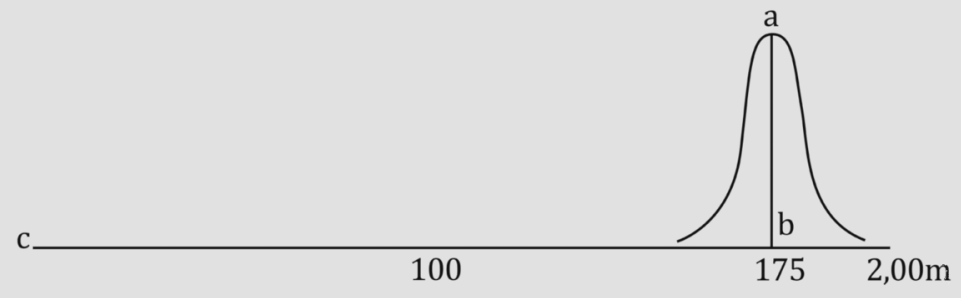

Quelle: Quetelet: Soziale Physik, Figur 1, S. 146.

Quetelet verwendet hier die Darstellungsform der Gaussschen Normalverteilung. Sie macht sichtbar, welche Werte am häufigsten auftreten und welche Abweichungen von der häufigsten Form in welcher Zahl vorgefunden werden.

Die Frage nach der Häufigkeitsverteilung ist nun der Punkt, an dem sich die beiden Traditionsstränge, die staatliche Statistik und die Wahrscheinlichkeitstheorie, getroffen haben. Wie es der staatlichen Statistik um die Häufigkeiten sozialer Phänomene ging, so den Wahrscheinlichkeitstheoretikern um die Häufigkeiten von Ergebnissen. Für beide ergab es sich, dass die gesuchte Regelmäßigkeit in der großen Zahl liegt: für die einen in der großen Menge sozialer Gegebenheiten, für die anderen in der großen Menge der Ereignisse (z.B. der Ziehungen von Kugeln). Jeweils stabilisiert sich der Wert des Gesamten mit der steigenden Zahl der Daten.

Was nun kann eine Wissenschaft an der Verteilung von Häufigkeiten interessieren? Dass diese Frage im Glücksspiel, im Versicherungswesen und in der Risikoabschätzung von Belang sind, liegt auf der Hand. Aber was hat dies mit Wissenschaft zu tun? 
Hier nun tritt das Thema der Ursachenforschung auf den Plan, mit ihrer gänzlich neuen Fragestellung in diesem Feld! Ursachenforschung bedeutet ja (auf die schlichteste Formel gebracht) zu fragen, unter welchen Bedingungen A auftritt und ob A ausbleibt, wenn eine bestimmte Bedingung nicht gegeben ist.

Wie hier, im Feld der Ursachenforschung, sich das Problem der Statistik weiterentwickelte, machen wir uns an einem Beispiel klar, das Ronald A. Fisher, einer der Gründungsväter der modernen Statistik, in seiner einführenden Darstellung benutzt. (Abb. 7)

$A b b .7$

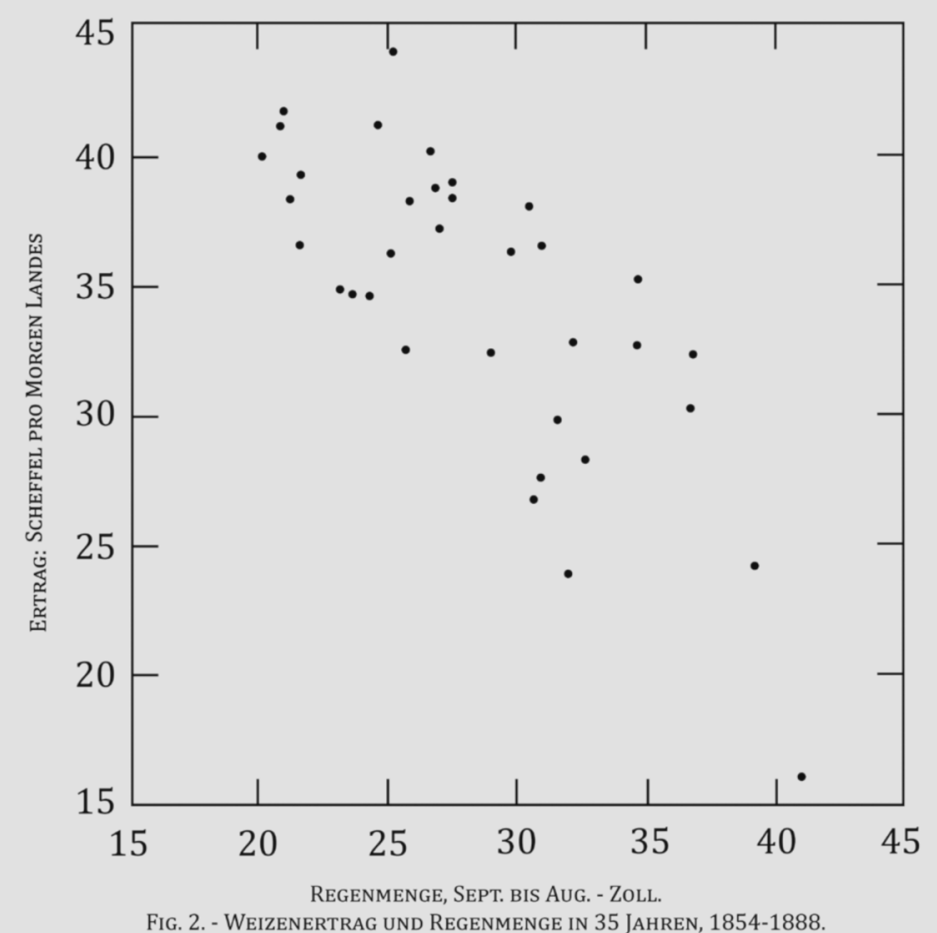

Quelle: Fisher: Statistische Methoden, S. 31.

Diese Darstellung zeigt ein Korrelations-Diagramm von Regenmengen und Ernteerträgen. Und auch hier gilt wie schon oben: den Punkten im Diagramm entsprechen keine unmittelbaren empirischen Gegebenheiten. Un- 
mittelbare Daten aufzuzeichnen, sähe in diesem Fall so aus, dass wir Jahr für Jahr die Regenmenge und Jahr für Jahr den Ernteertrag notieren. Diese Notate könnten als Punkte jeweils in ein Koordinatensystem eingetragen und zu Linien verknüpft werden. Die beiden Linien könnten sodann miteinander verglichen werden und man könnte nach Übereinstimmung in den Kurven suchen. Eine solche Darstellung stünde genau auf der Grenze zwischen Quetelets Ordnungserkenntnis in Punktmengen und einer ursachenbezogenen Sichtweise. Allerdings: Wie sich schon am Beispiel von Geburtenrate und Storchpopulation zeigte, können solche Korrelationen nur indirekt kausal oder sogar rein zufällig sein.

Fishers Punktmenge ist tatsächlich keine, in der man empirische Ordnungen dargestellt findet. Jeder einzelne dieser Punkte ist schon die Darstellung einer Verhältnisgröße. Er stellt weder den Ernteertrag noch die Regenmenge dar, sondern stets schon das Verhältnis von Ernteertrag und Regenmenge. Dieses Verhältnis ist erschlossen, nicht gegeben. In einem strengen Sinne haben wir es bei den Punkten daher nicht mehr mit Daten zu tun, vielmehr mit Resultaten von Operationen mit Daten. Das Suggestive besteht nun darin, dass die Punkte immer noch wie Daten aussehen. Es scheint so, als ob die Verknüpfung von Regenmenge und Ernteertrag eine empirische Tatsache ist. Die Suggestion kommt zustande, weil man dem Punkt nicht ansieht, dass er seinen Charakter wesentlich verändert hat. Es sieht so aus, als ob jeder Punkt eine reale Gegebenheit anzeigt und als ob alle Punkte zusammen eine Regelmäßigkeit in der Wirklichkeit anzeigten. Tatsächlich aber zeigen sie das Ergebnis einer In-Beziehung-Setzung an: Wenn wir den Ernteertrag zur Regenmenge in Beziehung setzen, dann erscheint das (hier sichtbare) Resultat.

Dieses Resultat kann aber aus unterschiedlichen Gründen eine Ordnung aufweisen. Zum einen ganz zufällig: In der Korrelation vieler empirischer Verläufe werden immer wieder zufällig ähnliche Verläufe und damit Gestalttendenzen in der Punktmenge auftreten (siehe Abb. in BASISWISSEN 12). Zum anderen, weil die >korrelierten Daten gar nicht unabhängig voneinander sind, sondern einem gemeinsamen Ursachenzusammenhang angehören, der aber nicht durchschaut wird (z.B., dass bei häufigem Regen die Bauern weniger Unkraut jäten o.ä.). Außerdem ist hier offen, ob nicht andere Faktoren, die nicht bedacht werden, mit dem Ernteertrag viel stärker in Beziehung stehen als die Regenmenge. Die Korrelationswerte kommen also durch eine Operation zustande, die auf der Annahme beruht, dass es eine bedeutende ursächliche Beziehung zwischen Ernteertrag und Regenmenge gibt. 
Entsprechend vorsichtig drückt sich Fisher an dieser Stelle auch aus:

Es »kann ein Punkt-Diagramm Assoziationen anregen, die bis dahin nicht vermutet worden waren, oder, was ebenso wichtig ist, die Abwesenheit von Assoziationen darstellen, die bis dahin zuversichtlich vorausgesagt worden waren. Der Wert der Punkt-Diagramme besteht darin, dass sie eine einfache Übersicht über die bisher gesammelten Erfahrungen und dem Ceist Anregungen geben, die experimentellen Untersuchungen zugänglich sind..166

Lesen wir genau, was Fisher hier schreibt: die Punkte im Diagramm geben Anregungen für Experimente. Statistik in diesem Sinne wäre ein Instrumentarium innerhalb experimenteller Forschung. Als heuristischer Einsatz kann sie der experimentellen Laborforschung Hinweise geben, aber sie ist als solche nicht geeignet, experimentelle Ergebnisse zu beurteilen.

Doch genau dieses Verhältnis zwischen mathematischer Datenbehandlung und experimenteller Forschung kehrt sich in der modernen Statistik um. Es scheint nun so, als ob mathematische Operationen mit Daten dazu geeignet wären, empirische Annahmen zu validieren, das heißt: darüber zu entscheiden, welche empirische Annahme in welchem Maße gerechtfertigt ist. Und damit auch: die Gültigkeit von Annahmen im Sinne des Grades von Wahrscheinlichkeit zu beweisen.

Am deutlichsten zeigt sich diese Aufladung des Anspruchs am sogenannten P-Wert, der im Zentrum der modernen Statistik - und der Debatte über sie - steht. Fisher hatte diesen Wert als ein Maß für die Abweichung von der Standard-Verteilung eingeführt. Graphisch betrachtet handelt es sich sozusagen um die Fläche des Kurvenendes der Häufigkeitsverteilung. (s. Abb. 8)

Es sind mehrere Aufstufungen, mit denen aus Daten ein P-Wert erzeugt wird. Im ersten Schritt werden - wie soeben gesehen - zwei Datensätze korreliert und zu einem vereinigt. Im zweiten Schritt wird diese resultierende Datenmenge nun daraufhin betrachtet, ob sich in ihr eine regelhafte (Un-) Gleichverteilung findet, sodass ein Regelzusammenhang erkennbar wird (z.B. je weniger Regen, desto mehr Ernte). Dargestellt wird dies, indem eine Linie in das Punktdiagramm eingezeichnet wird, wie beispielsweise in Abb. 9.

166 Ronald A. Fisher: Statistische Methoden für die Wissenschaft, Edinburg/London 1956 (12. neu bearb. u. erw. Aufl.) (im Orig. 1925), S. 31f. 
Abb. 8: Darstellung des p-Werts.

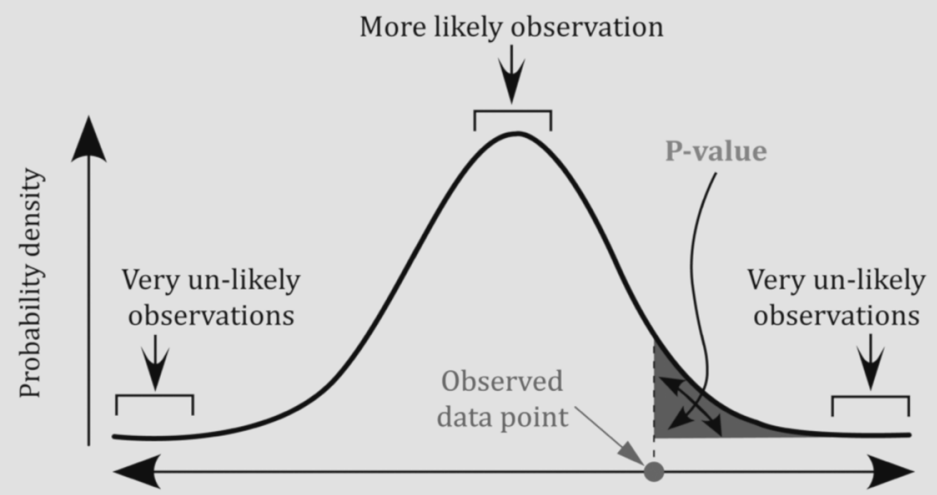

Set of possible results

A p-value (shaded green area) is the probability of an observed (or more extreme) result assuming that the null hypothesis is true.

Quelle: Wikipedia

Abb. 9: Linien-Darstellung von Korrelationen.

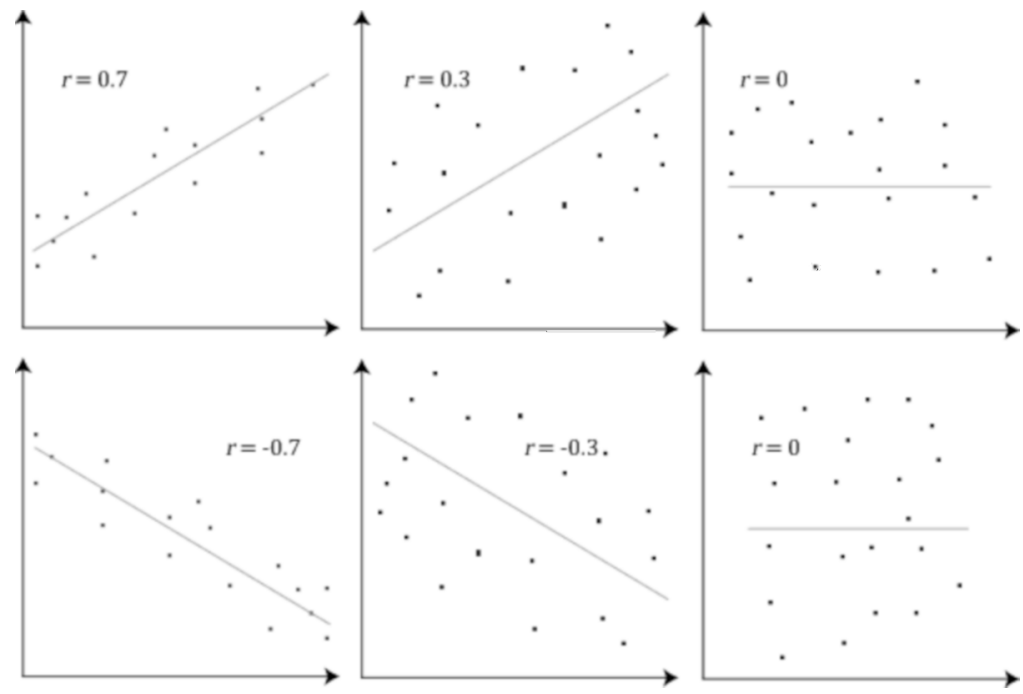

Quelle: Wikipedia 
Im dritten Schritt wird diese resultierende Regelannahme, die aufgrund von Daten gewonnen wurden, die aus einer Beziehungsannahme stammen (so kompliziert müssen wir das sagen!), in der Weise näher bestimmt, dass das Maß, in dem die einzelnen Daten (Punkte) der ermittelten Regel entsprechen, berechnet wird. Damit wird erschlossen, wie gut die einzelnen Daten zu der Regelmäßigkeit passen, die sich aus ihnen ergeben hat. Es wird also die Streubreite der Daten bestimmt. Im nächsten Schritt wird nun diese Streubreite verglichen mit der Streubreite von Daten, die schon aufgrund einer zufälligen Verteilung - oder aufgrund von Messfehlertoleranzen - zu erwarten gewesen wäre. Warum man dies tut, ist klar: Man möchte ein Maß für die Besonderheit der angenommenen Korrelation an die Hand bekommen, ein Maß, mit dem sich zeigen lässt, dass diese angenommene Korrelation den Bereich der zufälligen Streuung von Daten übersteigt. Dazu dürfen Werte, die außerhalb der normalen Abweichung liegen, nur relativ selten auftreten. Weil man diese relative Regelmäßigkeit nur im Vergleich erkennen kann, kehrt man die gesamte gedankliche Versuchsanordnung um: Man geht von der Annahme aus, dass sich in den zueinander in Beziehung gesetzten Größen gerade keine spezifische Korrelation findet - dass die abweichenden Werte also nur dem Zufall geschuldet sind (Nullhypothese) - und vergleicht die aus den Daten gewonnenen Abweichungswerte mit diesen zufallsbedingten: der P-Wert bemisst nun die Größe des Unterschiedes zwischen diesen beiden Abweichungssummen.

Die Debatte um den P-Wert dreht sich nun unter anderem um die Frage, wie groß dieser Unterschied sein muss, damit das Ergebnis signifikant ist. ${ }^{167}$ Doch abgesehen von solchen Fragen haben wie es hier bei diesem Vorgehen mit einigen wesentlich tiefer liegenden Problemen zu tun. Woher nämlich kennen wir denn die zzufällige Wahrscheinlichkeitsverteilung? In unserem Beispiel: Woher wissen wir denn, in welcher Streuung die Ernteerträge schwanken - abgesehen von Unterschieden in der Regenmenge? Wir müssten langfristige Messreihen zur Verfügung haben, in denen die Regenmenge konstant gehalten werden könnte. Damit ist klar, dass wir eine experimentelle Versuchsanordnung benötigen, die es erlaubt, Faktoren probeweise anund abzuschalten. Die Situation wird dadurch erschwert, dass wir ja auch aus langfristigen Messreihen nicht ersehen können, ob sie einer nur zufälli-

167 Vgl. Ronald L. Wasserstein/Nicole A. Lazar: >The ASA Statement on $p$-Values: Context, Process, and Purpose. In: The American Statistician, 2 (2016), S. 129-133. 
gen Streuung unterliegen - oder ob nicht vielmehr andere kausale Faktoren, die in ungewöhnlicher Weise auftreten (z.B. Schwankungen in der Bienenpopulation), eine ungewöhnliche Verteilung der Werte hervorrief.

Damit zeigt sich auch das Grundproblem der Anwendung der mathematischen Verfahren in der Statistik: Diese Verfahren sind aus Spielsituationen gewonnen, in denen die möglichen Resultate durch die geschlossene Struktur der Spielanlage bestimmt sind. Stellen wir uns vor, wie wir von einer Spielsituation wie dem Würfelspiel zu einer Problemstellung gelangen, wie sie sich in der Soziologie, der Medizin oder in den Agrarwissenschaften darstellt. Dazu müssten wir von einem Würfel ausgehen, der nicht nur zufällig und mit gleicher Wahrscheinlichkeit verschiedene Resultate liefert, sondern von einem, der unterschiedlichsten kausalen Einflüssen ausgesetzt ist, die jeweils die Tendenz der Ergebnisse beeinflussen; der sich also, in Abhängigkeit von vielen, selbst veränderlichen, teils womöglich unbekannten, teils interdependenten Faktoren immer wieder anders verhält. Regelmäßigkeiten wären zu beobachten, aber jede weitergehende Erklärung des Würfelverhaltens würde voraussetzen, dass das Bedingungsgefüge dieses Verhaltens im $\mathrm{Zu}$ sammenspiel seiner konstanteren und variableren Faktoren verstanden wäre.

Die an limitierten Spielsituationen gewonnene Mathematik ist somit einer bestimmten Idee von wissenschaftlicher Forschung entgegengesetzt: Während es in der - emphatisch aufgefassten - Forschung gerade darum geht, noch unerkannte oder unzureichend verstandene Faktoren aufzudecken, in ihrer Wirksamkeit und in ihrem Zusammenspiel theoretisch oder modellhaft zu erfassen, fragt dieser Typ von Statistik - gleichsam mechanisch - die Rolle von Einzelfaktoren ab. Man glaubte, auf ein Verständnis der Wirkungszusammenhänge verzichten zu können, weil ja - so schien es - sich an den Daten selbst Regelmäßigkeiten ablesen und das Maß der Regelmäßigkeiten bestimmen ließ. Doch dass in diesem Prozedere an mehreren Stellen quasi-theoretische Annahmen in Gebrauch genommen werden - erstens schon bei der Wahl der gemessenen Größen, zweitens bei der Wahl der untersuchten Korrelation und, drittens, bei der Bestimmung der Vergleichsebene - dass diese Statistik also nicht theoriefrei, sondern latent hochgradig theoretisch ist und dabei auf schwachen Beinen steht, das wurde übersehen. So absurde Ergebnisse, wie sie die P-Wert-geleitete Forschung produziert (indem z.B. eine signifikante Korrelation zwischen politischer Gesinnung und physiologischer Fähigkeit, 
Grauschattierungen wahrnehmen zu können, festgestellt wird). ${ }^{168}$ haben ihren Grund in einer Verfahrensweise, in der man meint, aus Daten Schlüsse auf Regeln ziehen zu können, ohne dazu irgendein Verständnis des Regelzusammenhangs entwickeln zu müssen. Das sogenannte P-Hacking ist die Endstufe einer Entwicklung, in der sich ,Wissenschaft‘ von Intelligibilität löst. ${ }^{169}$

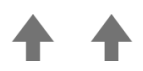

Unbenommen von der tiefliegenden Problematik probabilistischer Verfahren bleibt aber der Wert der Statistik im Allgemeinen. Geht es darum, Regelmäßigkeiten der Erscheinungen zu beobachten und aufgrund dieser Beobachtungen Prognosen anzustellen, so kann dieses Vorgehen einer kausalanalytischem auch überlegen sein. Steven Toulmin erläutert dies am Beispiel der - so von ihm angenommenen - Diskrepanz zwischen babylonischer und griechischer Astronomie. Während erstere aufgrund ihrer arithmetischen Kompetenzen »ein bedeutendes Vorhersagevermögen « ${ }^{170}$ ausbildeten, entwickelten zweitere durch »den Gebrauch der spekulativen Phantasie« eine »theoretische Basis«, die »zu einer Deutung der Himmelsbewegungen kommt « ${ }^{171}$, aber in Sachen Prognostik hinter ersterer zurückbleibt.

Ganz analog geht es bei den Clustering-Ansätzen in Big Data Analysen darum, potenziell relevante Muster zu erkennen, um Prognosen anstellen zu können. ${ }^{172}$ Bleibt dieser Ansatz innerhalb seiner Grenzen, dann hat er einen bedeutsamen praktischen Wert. Statistik zeigt sich dann als ein Mittel, Unterschiede und Gemeinsamkeiten in großen Datenmengen zu detektieren. Sind die Datenmengen klein, genügt das Gedächtnis oder einfache visuelle Darstellung, um solche Verhältnisse sichtbar und einschätzbar zu machen. Mit dem Anwachsen der Datenmenge verlieren wir diese Möglichkeit aber sehr schnell und es zeigt sich, wie sich die mathematische Statistik als ein Instrumentarium der Forschung bewährt: Wie das Teleskop und das

168 Regina Nuzzo: Fehlerschätzung. Der Fluch des P-Wertes. In: Spektrum Spezial 3 (2017) (zunächst in Heft 9 (2014)), S. 32-36, hier S. 32.

169 Vgl. Nuzzo, 2014, S. 32-36.

170 Stephen Toulmin: Voraussicht und Verstehen. Ein Versuch über die Ziele der Wissenschaft, Frankfurt a.M. 1968, S. 36.

171 Toulmin, 1968, S. 36.

172 Vgl. Harald Binder/Maria Blettner: Big data in medical science-a biostatistical view. In: Deutsches Ärzteblatt International 112, 9 (2015), S. 137-142. 
Mikroskop die Bereiche des Sehr-Entfernten und des Sehr-Kleinen zugänglich machen, so erschließt die mathematische Statistik den Bereich des SehrVielen, der großen Mengen.

Anders gesagt: Bleibt die Statistik in ihrem Selbstverständnis im Paradigma der Naturalis Historia, dann ist sie ein wertvolles Instrumentarium. Gibt sie aber vor, an wirklichem Experiment und sorgfältiger Interpretation vorbei Beweiskraft für Thesen bieten zu können, dann führt sie in die Irre und suggeriert eine Leistung, die sie nicht erbringen kann. 



\section{Die Pluralität der Wissenschaften: Reflexionen}

\section{Einteilungen von Wissenschaft 1: Frühe Abgrenzungen, Kants Systematisierung und die Bipolarisierung von Geistes- und Naturwissenschaften im 19. Jahrhundert}

Bevor wir uns der Frage zuwenden, wie Einteilungen von Wissenschaften bereits vorgenommen und wie historisch das Verhältnis von Wissenschaften untereinander reflektiert wurde, gilt es innezuhalten und zu fragen: Welche Art Forschung, welche Form des Wissens stellt denn das Aufweisen dreier Traditionen und die Auffächerung in neun Typen von Wissenschaft dar? Und wie rechtfertigt sich unser Herangehen?

Zunächst lässt sich feststellen: Traditionen nachzuzeichnen geschieht durch historische Rekonstruktion im Sinne des hermeneutischen Paradigmas: hin- und hergehend zwischen Teil und Ganzem verwendet diese Rekonstruktion das Bild der gegenwärtigen Wissenschaftslandschaft, um historisch Partikularität aufzuspüren und umgekehrt das historisch-partikulare Material, um das Bild der gegenwärtigen Gesamtlage zu korrigieren und $\mathrm{zu}$ reformulieren. Neben rekonstruktiven kommen aber auch typologisch-klassifizierende Verfahren im Einsatz: In einem Feld mit vielen Abschattungen und fließenden Übergängen werden bestimmte Merkmalskombinationen zentral gesetzt und zu Typen verdichtet; dabei werden auch vergleichende Verfahren verwendet und im Ergebnis zielt das Vorgehen auf eine übersichtliche Darstellung und Klassifikation. Aber auch Verfahren der Kritik sind im Einsatz: Denn eine Reflexion auf die eigenen Begriffe durchzieht die Darstellung von Beginn an: der Begriff von Wissenschaft als PraxisMotivationskomplex; Praxis ihrerseits als Konzept, das symboltechnische Formen, die normalerweise der Theorie zugeschlagen werden, inkludiert etc. Das Erkenntnisinteresse wurde ebenfalls expliziert: ein nicht-hierarchisches Bild unterschiedlicher Wissenschaftsformen. Auf reduktionistische Ver- 
fahren, die ein universales Regelwerk hinter den Erscheinungsweisen von Forschung aufzudecken oder zu postulieren versucht, wurde bewusst verzichtet: die Auffächerungen werden als kontingente Resultate historischer Ereignisse verstanden, als `faktische ( (gemachte) Gegebenheiten, nicht als notwendige Erscheinungen, hinter denen determinierende Regeln oder universale Gesetze stehen. Mit dieser Sichtweise beginnen wir schon, uns in die Fallstricke der Auseinandersetzung um Konstruktivismus und Realismus zu verwickeln, weshalb wir uns auch mit diesem Reflexionskomplex, mit dieser Debatte um Wissenschaft auseinanderzusetzen haben werden (im Kapitel 18: Deutungskämpfe II). Letztlich wäre für die Wissenschaften wohl am meisten gewonnen, wenn sich die hier vorgeschlagene Typologisierung als experimentelles Denken verstehen ließe: als Einsatz eines Experimentalaufbaus, in dem sich unterschiedliche Formen von Forschung aufweisen und unterscheiden lassen. Wir gelangen hier natürlich an die Grenzen der Darstellungsform Buch: zu einem experimentellen Setting kann es erst in Offenheit und Unabgeschlossenheit eines Diskurses werden, in dem sich die dargestellte Struktur an realen Forschungsphänomen bewährt oder durch sie zur Variation veranlasst wird.

Das wiederum verweist zurück auf die kritisch-hermeneutische Aufgabe, vorhergehende Einteilungen als denkexperimentelle Einsätze zu rekonstruieren und zu sehen, welche Resultate sie brachten:

Nicht überraschend wurde die Unterschiedlichkeit von Wissenschaften immer wieder zum Thema. Eher ist erstaunlich, dass dies weit weniger der Fall war, als es zu erwarten wäre. Und wo dies geschah, geschah es meist antagonistisch, also in kämpferisch-abgrenzender Weise. Ideale der Wissenschaft haben wenig Toleranz, aber viele Ansprüche auf Deutungshoheit hervorgebracht. Selten wurde Verschiedenartigkeit akzeptiert - und noch seltener wurde Unterschiedliches als möglicherweise koexistierend oder vereinbar angesehen. Und in der Folge davon wurde kaum je die Frage behandelt, wie verschiedene Arten von Wissenschaft füreinander und miteinander fruchtbar werden könnten. Doch rudimentär und verstreut gibt es Ausführungen zu diesen Punkten. Einigen sind wir schon begegnet, andere haben wir gestreift.

Der Kanon der Wissenschaften, wie er für die Neuzeit maßgeblich wurde, entstand aus der Tradierung der aristotelischen Schriften. Doch machen wir uns eines klar: einen Oberbegriff für alle Gebiete von Wissen und 
Könnerschaft gibt es bei Aristoteles nicht. Aristoteles baut seine Systematik auf der Unterscheidung der drei Felder episteme, praxis und poiesis auf. Im Feld des Herstellens (poiesis) werden Güter durch Könnerschaft (techné) hervorgebracht; im Feld der Praxis werden durch Klugheit (phronesis) erstrebenswerte Handlungsziele verwirklicht und im Feld der episteme wird das Seiende und seine Ursachen im Modus interessenloser Betrachtung (theoria) erkannt und durch Beweisführung (apodeixis) erschlossen. ${ }^{1}$ Dazu kommen Geist (nous), Weisheit (sophía), Erfahrung (empeiria) und weitere Spielarten des Intellekts. ${ }^{2}$ Diese intellektuellen Potenziale gliedern sich auf hinsichtlich der Unterscheidung, ob ihr Gegenstand als veränderlicher oder unveränderlicher erscheint und ob er einem äußeren Zweck als herstellbares Gut folgt (poiesis), oder ob seine Güte selbstzweckhaft ist (praxis). Auch hinsichtlich der Genauigkeit des jeweils erreichbaren Wissens unterscheiden sich die Erkenntnisformen, ${ }^{3}$ was wiederum davon abhängt, wie beständig ein Gegenstand ist, ob er selbständig und ob er stoffgebunden oder vom Stoff ablösbar ist. So handelt die Physik von selbständigen, beweglichen Gegenständen, die Philosophie von selbständigen und unbeweglichen und die Mathematik hauptsächlich von unselbständigen, unbeweglichen. ${ }^{4}$ Mathematik, Philosophie und Theologie gehören demnach zu den betrachtenden Wissensformen, während z.B. Medizin, Musik, Baukunst als herstellende Kenntnisformen und Ökonomie (Hausführungskenntnis) und Politik (Staatsführungskenntnis) zur Praxis gehören. Die Klugheit der Praxis und die Könnerschaft der Herstellung beziehen sich jeweils auf Veränderliches - also gerade nicht auf die unveränderlichen Prinzipien, von denen es epistemisches Wissen und Prinzipienwissen in Form von Weisheit geben kann. So fächert Aristoteles das Spektrum der Kenntnisse hinsichtlich der Kriterien von Veränderlichkeit und Unveränderlichkeit und von Autonomie und Heteronomie auf. Als Ganzes ergibt dies ein System, das Kenntnis, Können und Wissen facettenreich und abgestuft entfaltet, und zwar jeweils gemäß der Prinzipien, die in einem Sachgebiet herrschen sowie der Frage, wie stark ein Erkenntnisgegenstand durch unveränderliche Prinzipien gebunden ist.

1 Aristoteles: Nikomachische Ethik. 6. Buch, 3-5; 1139b-1140b.

2 Aristoteles: Nikomachische Ethik. 6. Buch, 10-13; 1142a-1145a.

3 Aristoteles: Metaphysik, 2. Buch, 3; 995a; Nikomachische Ethik. 1. Buch, 1; 1094 b.

4 Aristoteles: Metaphysik, 6. Buch, 1;1026a. 
Wir sind heute geneigt - und die modernen Übersetzungen verfahren entsprechend - all diese Formen von Können, Kenntnis und Wissen als Wissenschaften anzusprechen. Doch damit wird man der Komplexität und der Integration sehr unterschiedlicher Formen des Intellektuellen in eine Ordnung von Erkenntnisformen nicht gerecht.

Diese Unterbietung hat ihre Hauptquelle in der Zuspitzung auf das Paradigma des Beweisens, die durch die Rezeption der Zweiten Analytik zustande kam. Dabei ist zu beachten: das logische Schließen, die Syllogistik, gilt bei Aristoteles als keine der Formen von Erkenntnis. Sie gilt vielmehr als ein Werkzeug des Erkennens, worauf auch der spätere Titel Organon für die logischen Schriften des Aristoteles hinweist. Mit Wissen verbunden ist Logik nur, wenn sie von an sich schon wahren Prämissen ausgeht; dann bildet sie als Beweisen (apodeixis) die Wissensform der episteme aus; immer vorausgesetzt, dass die ins logische Schließen eingehenden Voraussetzungen selbst schon auf der Grundlage von Erfahrungsbildung geistig durchschaut sind. ${ }^{5}$

Die aristotelische Erkenntnistheorie wurde über mehrere Stationen maßgeblich für die neuzeitliche Wissenschaftsauffassung. Eine zentrale Rolle spielte die arabische Tradition und Interpretation. Der Logiker und Grammatiker Al Farabi (ca. 870-950) hatte unter einem Titel, der wörtlich etwa Einteilung des Wissens bedeutet und der später unter De Scientiis verhandelt wurde, die aristotelische Wissenschaftstheorie mit arabischen Denkdisziplinen vereinigt. Hintergrund ist ein ganzer Kanon von Schriften, in denen es immer wieder um die Frage ging, wie maßgeblich die aristotelische Wissenschaftstheorie und Logik für Wissensgebiete sein kann, die auf anderen Sprachen als dem Griechischen beruhen. ${ }^{6} \mathrm{Al}$ Farabi war stark durch die Zweite Analytik geprägt, doch auch für ihn stellt diese Spannung ein zentrales Gliederungsmotiv dar. In Reaktion darauf baut er kulturspezifische Disziplinen wie Sprachwissenschaften, Rechtslehre und Theologie in die Darstellung des Kanons der aristotelischen Wissenschaftstheorie ein ${ }^{7}$ und gliedert sein Buch folgendermaßen:

5 Aristoteles: Nikomachische Ethik. 6. Buch, 6. 1140b-1141a. Aristoteles: Zweite Analytik. Analytica Posteriora, 2. Buch, Kap. 19; 99b-100b.

6 Vgl. Franz Schupp: Einleitung. In: Al-Fārābī: Über die Wissenschaften. De scientiis. Nach der lateinischen Übersetzung Cerhards von Cremona, mit einer Einl. u. kommentierenden Anmerkungen hrsg. und übers. v. Franz Schupp, Hamburg 2005, S. XI-LXXXIV, hier S. XIXXLVI.

7 Schupp, 2005, S. XXXVff. 
»Das erste Kapitel handelt also von der Wissenschaft der Sprache und deren Teilen.

Das zweite Kapitel handelt von der Wissenschaft der Logik und deren Teilen.

Das dritte Kapitel handelt von den mathematischen Wissenschaften. Dies sind die Arithmetik, die Ceometrie, die Optik, die mathematische Astronomie, die Wissenschaft der Musik, die Wissenschaft von den Gewichten und die [technische] Erfindungswissenschaft.

Das vierte Kapitel handelt von den Naturwissenschaften und deren Teilen und von der göttlichen Wissenschaft und deren Teilen.

Das fünfte Kapitel handelt von der Staatswissenschaft und deren Teilen, von der Rechtswissenschaft und von der Wissenschaft der Beredsamkeit. ${ }^{8}$

Dieses Buch wurde in Toledo von Gerhard von Cremona (1114-1187) unter dem Titel De Scientiis ins Lateinische übersetzt und von Dominicus Gundissalinus als Grundalge seines Hauptwerks De divisione philosophiae verwendet. ${ }^{9}$ Es traf in dieser Zeit auf eine Situation, in der die

»traditionelle Einteilung der Wissenschaften im Trivium (Grammatik, Logik, Rhetorik) und Quadrivium (Arithmetik, Ceometrie, Musik, Astronomie) nicht mehr ausreichte. Schon vor dem Bekanntwerden der neuen Texte durch die in Toledo hergestellten Übersetzungen aus dem Arabischen hatte Hugo von St. Viktor in seinem um 1127 verfaßten Didascalion eine neue Wissenschaftseinteilung vorgenommen, in der neben den traditionellen theore-

8 Al-Fārābī: Über die Wissenschaften. De scientiis. Nach der lateinischen Übersetzung Gerhards von Cremona, mit einer Einl. u. kommentierenden Anmerkungen hrsg. und übers. v. Franz Schupp, Hamburg 2005, S. 3.

9 Alexander Fidora: >Aristotelische Wissenschaft als Netzwerk von Wissenschaften. Die Rezeption der aristotelischen Wissenschaftstheorie bei al-Fārābī und Domenicus Gundissalinus.< In: Ludger Honnefelder (Hrsg.): Albertus Magnus und der Ursprung der Universitätsidee. Die Begegnung der Wissenschaftskulturen im 13. Jahrhundert und die Entdeckung des Konzepts der Bildung durch Wissenschaft, Weilerswist 2017, S. 77-96, hier: S. 79. 
tischen Wissenschaften auch praktische und mechanische Wissenschaften eingeführt wurden. ${ }^{10}$

Wir sehen: die Zeit war reif, die aristotelische Auffächerungen der Kenntnisse vom Herstellen, vom Klug-Handeln und von den Prinzipien des Seienden unter einen vereinheitlichenden Begriff zu fügen. Das geschah nun insbesondere durch Gundissalinus' De divisione philosophiae. Dessen

»systematische Bedeutsamkeit [gründet] in der Tatsache, dass die Divisionsschrift mit ihrer Bestimmung der Philosophie, ihrer Einteilung der Wissenschaften sowie der letztlich aristotelischen Beschreibung ihres epistemologischen Status und ihrer Vernetzung das Wissenschaftsverständnis nicht nur seinem Inhalt, sondern auch seiner Form nach neu gestaltet. «11

Doch in dieser Adaption und Auffächerung wissenschaftlicher Disziplinen fand gerade keine Unterteilung wissenschaftlicher Typen statt. Vielmehr wurden die Disziplinen allesamt in das Paradigma der Beweislehre gefügt, wodurch sich dieser spezifische Begriff von Wissenschaftlichkeit erst ausprägte.

Doch weil die episteme als Beweislehre bei Aristoteles gerade nicht die Rolle eines autonomen Wissens spielt, das alle anderen Wissensformen beherrscht, sondern der Erfahrungsbildung (empeiria), der geistigen Einsicht (nous) und der Prinzipienreflexion (sophia) bedarf, führte die Aristotelesinterpretation in der Folge zu einem ersten typenprägenden Gegensatz: dem zwischen rationalen und empirischen Erkenntnisweisen. (Siehe auch oben BASISWISSEN 6: Induktion und Empirie - Der Weg der Erfahrung). Bei Aristoteles in eine wohlgefügte Ordnung des Erkenntniszusammenhangs eingepasst, wurden diese beiden Modi in den Debatten an der Grenze von Hochmittelalter und früher Neuzeit zu scheinbar alternativen Erkenntnisformen. Mit der Dominanz des Beweisparadigmas (apodeixis) erschien Erkenntnis als ein Verfahren, das seinen Platz im Wesentlichen innerhalb der Rationalität als einer Form des Schlussfolgerns fand. Dagegen opponierten diejenigen Aristoteles-Kenner, die seine Erkenntnislehre als wesentlich durch Induktion (epagogé) und Erfahrung (empeiría) geleitet verstanden. Für sie war Erkenntnis niemals ein Operieren in Denkformen, sondern immer gebunden an ein Wahrnehmen der erfahrbaren Welt.

10 Schupp, 2005, S. LXIXf.

11 Fidora, 2017, S. $79 f$. 
Dieser Gegensatz war über Jahrhunderte virulent und schlug sich noch $\mathrm{zu}$ Beginn des 17. Jahrhunderts bei Francis Bacon nieder, der nun hoffte, "zwischen der beobachtenden und denkenden Fähigkeit - deren [...] Trennung alles in der menschlichen Familie verwirrt hat - eine wahre und rechtmäßige Ehe für alle Zeiten begründet zu haben ${ }^{12}{ }^{12}$ Und auch hier gilt es, genau auf die Worte zu sehen. Denn in der Übersetzung mit beobachtend wird leicht übersehen, dass Bacon von einer Verbindung »inter empiricam et rationalem facultatem ${ }^{13}$ spricht - also zwischen der empirischen und der rationalen Fähigkeit - und damit von dem, was Aristoteles unter Erfahrung verstand: nicht bloße sinnliche Wahrnehmung, sondern auch ein praktischkonzeptuelles Vermögen, die Gemeinsamkeiten im Vielfältigen zu erkennen.

Und ebenfalls haben wir bereits angesprochen, dass Bacons Ansatz sich programmatisch stark von dem zeitgleichen Galileis und dem ebenfalls zeitgleichen Descartes unterschied. Galilei und Descartes heben auf ratio als formal-mathematisches Verfahren ab, und degradierten das Experimentieren zur Hilfsdisziplin, während Bacon umgekehrt von der Überzeugung geleitet war, dass erfahrungsfreies Schlussfolgern niemals zur wirklichen Erkenntnis führen kann.

So wurde im frühen 17. Jahrhundert der ältere Gegensatz zwischen »via experientiae " und »via rationis", zwischen "ratio et experimentum «" überschrieben durch den neueren Gegensatz zwischen einer Präferenz des formal-mathematischen und einer Präferenz des technisch-experimentellen Erkenntnisweges.

Unabhängig davon entwickelten, wie wir sehen konnten, die Verstehenslehren eigene systematische und methodische Ideale. Das Forschungsparadigma der Hermeneutik, das im Hin- und Hergehen zwischen Teil und Ganzem Zusammenhänge rekonstruiert und auslegt; das Adaequationsparadigma mit seinem Ideal der Anpassung der Konzepte an den Gegenstand und das Paradigma der Kritik, das Erkenntnis an die Klärung der Bedingtheiten und Voraussetzungen band. Der hermeneutische Blick auf Forschung zeigte sich in seinen Momenten des Suchens und Versuchens eng verwandt

12 Francis Bacon: Neues Organon, Hamburg 1999, S. 31.

13 Bacon, 1999, S. 30.

14 Theodor W. Köhler: Sachverhaltsbeobachtung und axiomatische Vorgaben. Zur Struktur wissenschaftlicher Erfassung konkreter Äußerungsweisen des Menschlichen im 13. Jahrhundert. In: Erfahrung und Beweis. Die Wissenschaften von der Natur im 13. und 14. Jahrhundert, hrsg. v. Alexander Fidora und Matthias Lutz-Bachmann, Berlin 2009, S. 125150, hier S. 129-133. 
mit dem Zweig der Scientia-Tradition, der sich als Experimentalismus ausprägte: suchen, probieren, versuchsweise integrieren - ein spiralförmiger Forschungsprozess.

Und die Traditionslinie der Naturgeschichte: in ihren Zugängen des Beschreibens und Typologisierens prägten auch sie markante wissenschaftliche Paradigmen aus. In dieser Linie stehen die Verfahren des Datensammelns und -verarbeitens, die wir als statistisches Paradigma besprochen haben. Und auch hier finden sich wieder Verwandtschaften: Während die sich zurücknehmende Geste des Beschreibens Ähnlichkeiten mit dem Kritikparadigma der Verstehenslehren zeigt, weist die probabilistische Spielart des statistischen Denkens zurück auf das Paradigma der mathematisierten episteme.

So deuten sich einige Berührungspunkte und Trennungslinien an, die allerdings bis ins 19. Jahrhundert kaum wahrgenommen wurden. Man unterschied zwar Disziplinen und Teilbereiche der Erkenntnis, gliederte den Baum der Wissenschaften auf verschiedene Weisen, sah sie aber stets als einen Zusammenhang mit gemeinsamen Wurzeln. Wenn Descartes und Leibniz in Anschluss an Raimundus Lullus eine scientia generalis entwerfen, dann bewegen sie sich zwar in einer Sphäre rein rationalen Kombinierens und Schließens, aber sie verstehen dies gerade als integratives Prinzip, nicht als Kriterium, um verschiedene Typen von Wissenschaft voneinander abzusetzen. Sicherlich finden wir bei Galilei und auch bei Bacon Polemiken gegen scholastisches Denken, gegen die Tradition des Aristotelismus, aber diese Absetzbewegungen dienten mehr dazu, Raum für die Mathematisierung und die Experimentalisierung der Wissenschaften zu schaffen, als dass sie unterschiedliche Wissenschaftsideale gegeneinander in Stellung brachten. Noch als d'Alembert 1743 die einer »Bewegung inhärierenden Kräfte« als »dunkle, der Metaphysik angehörige Begriffe $«^{15}$ verbannte, ließ sich daran zwar die Idee einer deskriptiven Wissenschaft knüpfen, doch war dies im Kern - wie wir sahen - vielmehr ein Schritt auf dem Weg in die mathematische Darstellung und stand völlig innerhalb der Konsequenz der Beweislehren.

15 Jean d'Alembert: Abhandlung über Dynamik; in welcher die Gesetze des Cleichgewichts und der Bewegung der Körper auf die kleinstmögliche Zahl zurückgeführt und in neuer Weise abgeleitet werden, und in der ein allgemeines Princip zur Auffindung der Bewegung mehrever Körper, die in beliebiger Weise aufeinander wirken, gegeben wird, Übers. u. Anm. v. A. Korn, Thun u. Frankfurt a.M. 1997 (im Orig. 1743), S. 13. 
Näher an der Problematik unterschiedlicher Typen von Wissenschaft befindet sich Kants in den 1790er Jahren verfasste Schrift Der Streit der Fakultäten. Das Wort Fakultät, das, wie wir soeben sehen konnten, noch bei Bacon auch Vermögen, Fertigkeit, Könnerschaft meint, bezeichnet seit dem Mittelalter auch die unterschiedlichen Fächer, die an Universitäten vertreten sind. Kants Schrift geht von der etablierten Unterteilung in die oberen Fakultäten der Theologie, Jurisprudenz und Medizin und die vierte, untere Fakultät aus, die als Vorstudium (Propädeutikum) diente und die spätantike Einteilung der Septem Artes (Sieben freien Künste) zunächst übernahm. Bei Kant umfasst diese untere, sphilosophischeく Fakultät

»zwei Departemente, das eine der historischen Erkenntnis (wozu Ceschichte, Erdbeschreibung, gelehrte Sprachkenntnis, Humanistik mit allem gehört, was die Naturkunde [...] darbietet); das andere der reinen Vernunfterkenntnisse (reinen Mathematik und der reinen Philosophie, Metaphysik der Natur und der Sitten). $\ll^{16}$

Wir treffen hier also noch nicht den Gegensatz von Natur- und Geisteswissenschaften an, sondern den zwischen einer oberen Fakultät und einer unteren Fakultät (letztere mit ihren zwei Departementen). Was ist hier das wesentliche Unterscheidungsmerkmal? Kant bestimmt dies funktional: die oberen Fakultäten richten sich auf den Nutzen - und zwar im Sinne der Regierung: »Die Regierung aber interessiert am meisten, wodurch sie sich den stärksten und dauerndsten Einfluss aufs Volk verschafft $\aleph^{17}$. Und das strebt sie, so Kant, auf drei Ebenen an: durch die Theologie nimmt sie Einfluss auf das Denken, durch die Jurisprudenz auf das Verhalten, durch die Medizin auf Gesundheit und Wachstum des Volkes. ${ }^{18}$ Damit ist Sinn und Zweck der oberen Fakultäten beschrieben. Die der unteren nun bestimmt Kant auf eine eigene Weise. Sie nämlich habe die Aufgabe, die oberen zu »kontrollieren «. ${ }^{19}$ Und zwar

16 Immanuel Kant: Der Streit der Fakultäten (1798). In: ders.: Schriften zur Anthropologie, Geschichtsphilosophie, Politik und Pädagogik 1, Werkausgabe Bd. XI, hrsg. v. Wilhelm Weischedel, Frankfurt a.M. 1977, S. 261-393, hier S. 291.

17 Kant, 1977, S. 281.

18 Vgl. Kant, 1977, S. 283.

19 Kant, 1977, S. 290. 
»erstreckt« sich das Aufgabengebiet der unteren Fakultät auf alle Teile des menschlichen Wissens (mithin auch historisch über die obern Fakultäten), nur daß sie nicht alle (nämlich die eigentümlichen Lehren oder Gebote der obern) zum Inhalte, sondern zum Gegenstand ihrer Prüfung und Kritik [...] macht. «²0

Mit dieser Gliederung der Wissenschaftsbereiche etabliert Kant gleich zweierlei: erstens den Unterschied zwischen nutzenorientierter (wir würden heute sagen: anwendungsbezogener) und wahrheitsorientierter (grundlagenbezogener) Wissenschaft und zweitens die Bestimmung von Wahrheitsorientierung durch die Verfahren der Prüfung und Kritik; ein Ideal, das charakteristisch ist für ein Paradigma von Wissenschaft, das bestehendes Wissen durch Rückfrage auf seine Grundlagen und Berechtigungen in Wissenschaft überführt: das Paradigma der Kritik (Kapitel 13).

Warum aber geriet Kants Einteilung und Aufgabenverteilung so schnell und so gründlich in Vergessenheit? Hat es damit zu tun, dass das Paradigma der Kritik insgesamt so sehr im Verborgenen blieb?

Es waren jedenfalls zwei andere Paradigmen, die sich in der ersten Hälfte des 19. Jahrhunderts in den Vordergrund drängten. Da war zum einen der Positivismus, wie er zunächst im Cours de Philosophie Positive von Auguste Comtes (6 Bd. 1830-1842), sodann in A System of Logic (1843) von John Stuart Mill ausgearbeitet wurde. In beiden Werken geht es letztlich um eine Verortung der entstehenden Sozialwissenschaften im Kanon der etablierten Wissenschaften; beide Ansätze zielen dabei auf eine Integration in die Tradition der Beweislehre.

Comte bevorzugt die mathematische Variante, indem er als »das allgemeine Milieu aller möglichen Erscheinungen [...] die einfache mathematische, das heißt geometrische oder mechanische Daseinsweise, die allem realen Daseienden gemeinsam ist ${ }^{21}$, postuliert. Davon ausgehend kommt er zu einer Einteilung der Wissenschaften, die von der Mathematik in absteigender Allgemeinheit über Physik (darin zunächst die Astronomie), Chemie (zunächst anorganischer dann organischer) und Biologie bis zur Soziologie reicht. Dieser abnehmenden »Allgemeinheit und Unabhängigkeit« ent-

20 Kant, 1977, S. 391.

21 Auguste Comte: Rede über den Ceist des Positivismus, übers., eingel. u. hrsg. v. Iring Fletcher, Hamburg 1994, S. 107. 
spricht umgekehrt eine »zunehmende Kompliziertheit, aus der immer weniger abstrakte und schwierigere Theorien hervorgehen, aber auch immer bedeutendere und vollständigere auf Grund ihrer näheren Beziehung zum Menschen oder vielmehr zur Menschheit, dem abschließenden Gegenstande des ganzen theoretischen Systems. ${ }^{22}$

Abb. 10: Tableau Synoptique.

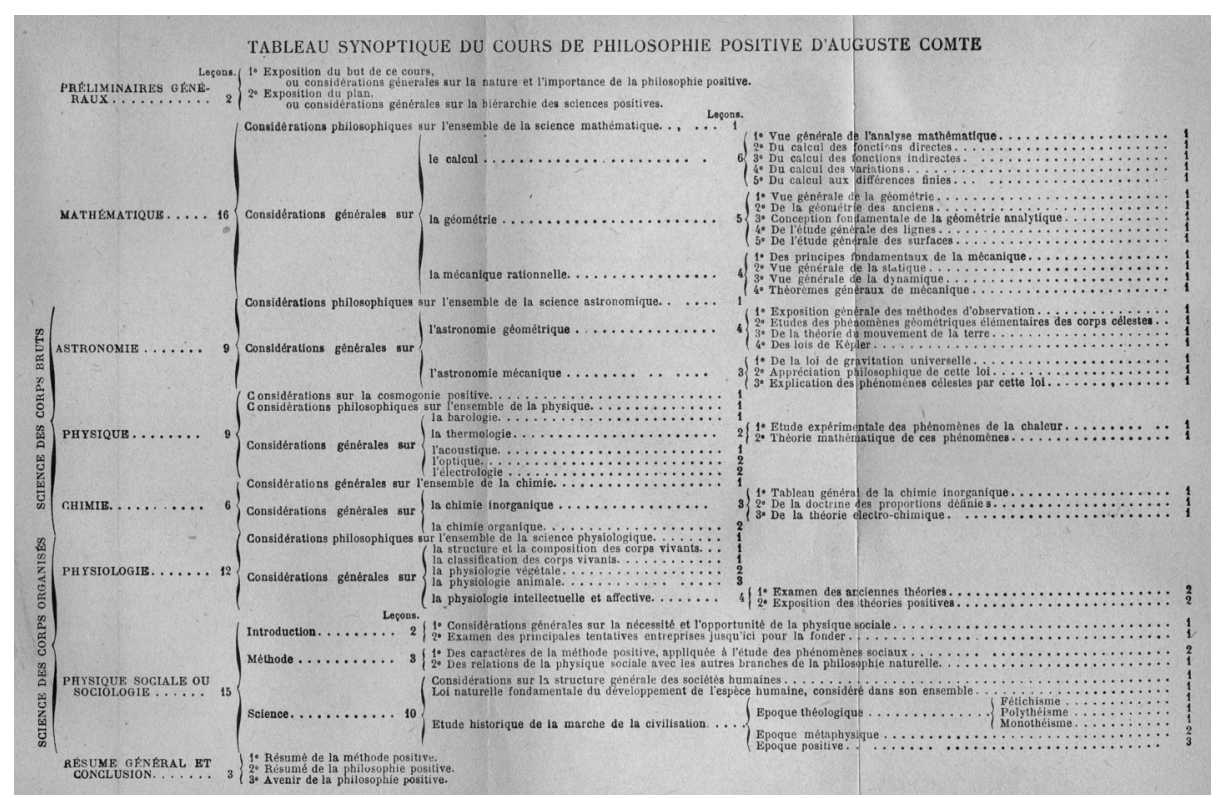

Quelle: Auguste Comtes Cours de Philosophie Positive.

Das Musterbeispiel einer Wissenschaftstheorie, die sich in die Kontinuität der klassischen, auf Logik und Syllogistik beruhenden Beweislehre stellt, ist John Stuart Mills A System of Logic. Für Mill gliedern sich die Wissenschaften danach, inwieweit es ihnen gelingt, vom induktiven Vorgehen zum Deduktiven überzugehen. Induktion übersetzt Mill - vielleicht der konsequenteste Vertreter der Logik-Variante der Beweislehren - in eine Form des induktiven Schließens: »Zu dem Zwecke der vorliegenden Untersuchung kann man die Induction definiren als: das Verfahren, durch welches man allgemeine Urt- 
heile (Sätze) entdeckt und beweist. « $^{23}$ Dass aber Induktion auch bei Mill bloße Generalisierungen sind und keine eigene Beweiskraft haben, zeigt sich, wenn als Beispiele Aussagen wie »die Säuren röthen blaue Pflanzenstoffe und die Alkalien färben sie grün « anführt. ${ }^{24}$ Der Umschlag zur Deduktion erfolgt, wenn sich eine ganze Reihe von einzelnen Generalisierungen in einem System verknüpfen, sodass wir nicht nur »die Leiter von a zu e durch ein syllogistisches Verfahren hinaufsteigen; wir können schliessen, dass a ein Merkmal von e ist, und ein jeder Gegenstand, der das Merkmal a hat, auch die Eigenschaft e besitzt«. ${ }^{25}$ Newtons Theorem der Kreisbewegung ist für Mill ein Beispiel eines solchen deduktiven Settings. ${ }^{26}$ Die Chemie hat, im Unterschied zur Physik, diesen Stand einer exakten Wissenschaft in Mills Augen noch nicht erreicht, ist jedoch dazu imstande. Das gleiche gelte für die »Sociologie«, die, gemäß der »Complexität« ihrer Materie, »eine deductive Wissenschaft nicht in der That nach dem Vorbilde der Geometrie, sondern nach dem Vorbilde der verwickelteren physikalischen Wissenschaften« sei. »Kurz, ihre Methode ist die concrete deductive Methode, wovon die Astronomie das vollkommenste, die Physik ein etwas weniger vollkommenes Beispiel

23 John Stuart Mill: System der deduktiven und induktiven Logik. Eine Darlegung der Prinzipien wissenschaftlicher Forschung, insbesondere der Naturforschung, 0.O. 2017, S. 507.

24 Das Zitat im Kontext: „Der Gegensatz besteht nicht zwischen den Ausdrücken Deductiv und Inductiv, sondern zwischen Deductiv und Experimentell. [...] Wenn wir durch unsere verschiedenen Inductionen nichtüberSätzehinausgekommen sind, wiediese: a ein Merkmal von b, oder a und b Merkmale von einander, c ein Merkmal von d, oder c und d Merkmale von einander, ohne den Zusammenhang von a und b mit c und $d$ zu kennen: so haben wir eine Wissenschaft von gesonderten und gegenseitig unabhängigen Generalisationen, etwa wie diese, die Säuren röthen blaue Pflanzenstoffe und die Alkalien färben sie grün. Aus keinem dieser Sätze könnten wir den andern direct oder indirect folgern, und soweit eine Wissenschaft aus solchen Sätzen besteht, ist sie rein experimentell. Die Chemie hat in ihrem gegenwärtigen Zustand diesen Charakter noch nicht abgelegt.« (Mill 2017, S. 407)

25 Mill, 2017, S. 408.

26 Mill schreibt: "So entdeckte z.B. Newton, dass sowohl die regelmässigen als auch die anscheinend anomalen Bewegungen aller Körper des Sonnensystems (eine jede dieser Bewegungen war durch eine besondere logische Operation aus besonderen Merkmalen gefolgert worden) Merkmale einer Bewegung um einen gemeinschaftlichen Mittelpunkt mit einerCentripedalkraft sind, welche in geradem Verhältniss der Massen und umgekehrt wie die Quadrate der Entfernungen von diesem Mittelpunkt variirt. Dies ist das grösste je vorgekommene Beispiel einer plötzlichen Umwandlung einer bis dahin bis zu einem hohen Grad bloss experimentellen Wissenschaft in eine deductive.« (Mill 2017, S. 427). 
darbietet, und deren Anwendung bei der von dem Gegenstande erheischten Vorsicht und Anpassung die Physiologie umzugestalten beginnt. « $^{27}$

Für die Geschichtswissenschaften schließt sich Mill dem Historiker Henry Thomas Buckle (1821-1862) an, insofern dieser statistische Gleichförmigkeiten als Gesetze der Geschichte interpretiert, die sich der Methodologie von Induktion und Deduktion unterwirft. ${ }^{28}$

Beide, Comte und Mill, integrieren somit sämtliche Wissenschaften in die Tradition der Beweislehre, mit der sie sie tatsächlich identifizieren. Dass es grundsätzlich andere Formen von Forschung gibt, die auf anderen Grundlagen stehen und auf andere Ergebnisse zielen, kommt ihnen nicht in den Sinn.

Es war der uns schon öfter begegnende Historiker Johann Gustav Droysen, der in einer Beilage mit dem Titel Erhebung der Geschichte zum Rang einer Wissenschaft in scharfzüngiger Kritik an eben jenem englischen Kollegen Henry Thomas Buckle eine gleichberechtigte Vielfalt wissenschaftlicher Methoden und zugleich einen wissenschaftlichen Verstehensbegriff forderte. Buckle postuliere die scheinbare Alternative: "Sind die Handlungen der Menschen und folglich auch der Gesellschaft bestimmten Gesetzen unterworfen, oder sind sie das Ergebnis entweder des Zufalls oder einer übernatürlichen Einwirkung? « Darauf Droysen: »Jawohl: diese Wolke ist, wenn nicht ein Kamel, so entweder ein Wiesel oder ein Walfisch.« Und er erläutert:

»Wir haben schon früher bemerkt, daß, wenn es eine Wissenschaft der Ceschichte geben soll, diese ihre eigene Erkenntnisart, ihren eigenen Erkenntnisbereich haben muß; wenn anderweitig die Induktion oder die Deduktion

27 Mill, 2017, S. 1651-1652.

28 »Die Lehre, [...] dass die Gesammtreihe der socialen Erscheinungen, mit anderen Worten, der Gang der Geschichte allgemeinen Gesetzen unterworfen ist, [...] ist durch das wichtige Werk des Herrn Buckle ausserordentlich gefördert worden.« (1724) Und weiter: »Nehmen wir die Wahrheit des Cesetzes an, so ist eine jede menschliche Handlung, eine jede »Mordthat zum Beispiel, das concurrirende Resultat zweier Reihen von Ursachen. Von der einen Seite die allgemeinen Umstände des Landes und seiner Einwohner; die moralischen Einflüsse, die Einflüsse der Erziehung, die ökonomischen und andere auf das ganze Volk einwirkende und den sogenannten Zustand von Civilisation ausmachende Einflüsse. Von der andern Seite die grosse Mannigfaltigkeit von Einflüssen, die dem Individuum besonders angehören, sein Temperament und andere Eigenthümlichkeiten der Organisation, seine Verwandtschaft, seine gewöhnlichen Genossen, Versuchungen u.s.f.«(Mill, 2017, S. 1728f.). 
vortreffliche Resultate ergeben hat, so kann das nicht die Folge haben, daß die Wissenschaft der Geschichte sich entweder des einen oder des anderen Verfahrens bedienen müsse; und glücklicherweise gibt es zwischen Himmel und Erde Dinge, die sich zur Deduktion ebenso irrational verhalten wie zur Induktion, die mit der Induktion und dem analytischen Verfahren zugleich die Deduktion und die Synthese fordern, um in der alternativen Betätigung beider nicht ganz, aber mehr und mehr, nicht vollständig, aber annähernd und in gewisser Weise erfaßt zu werden, die nicht entwickelt, nicht erklärt, sondern verstanden werden wollen. ${ }^{29}$

Der entscheidende Punkt ist für Droysen, dass sich geschichtliche Tatsachen nicht notwendig aus dem Vorhergehenden ergeben. Wir können sehen, wogegen Droysen sich wehrt: Es ist die Konzeption der apodeixis, die besagt, dass sich aus wahren Gründen das Folgende mit Notwendigkeit erschließen lässt. Solche Anfänge, wie sie die aristotelischen Prinzipien für die Schlussfolgerung darstellen, gibt es in der Geschichte aber nicht: »es ist einfach eine methodische Gedankenlosigkeit, wenn man forschend $\mathrm{zu}$ einem Punkt gelangen $\mathrm{zu}$ können meint, der anders als relativ der Anfang wäre, d.h. der sich anders als in dem, was daraus geworden ist, als Anfang ausweist, den wir also im Verhältnis zu diesem daraus Gewordenen als Anfang setzen. « ${ }^{30}$ Droysens eigenen methodischen Zugang hatten wir als eine besondere Konzeption von Analyse kennengelernt: "gleichsam ein Lockermachen und Auseinanderlegen« des historischen Materials »nach der ganzen Fülle seiner Momente, der zahllosen Fäden, die sich zu einem Knoten verschürzt habe. « $^{31}$ Die Fäden auseinanderziehen und aus den somit differenzierten Faktoren ein Geschehen »rekonstruieren $\aleph^{32}$ - das ist der Weg der interpretierenden Wissenschaft nach Droysen.

Für die weitere Entwicklung hin zu dem uns bekannten Gegensatz von Erklären und Verstehen war maßgeblich, wie Droysen die Eigentümlichkeit der beiden wissenschaftlichen Perspektiven näher charakterisierte.

\footnotetext{
29 Johann Gustav Droysen: Historik. Rekonstruktion der ersten vollständigen Fassung der Vorlesungen (1857), Grundriß der Historik in der ersten handschriftlichen (1857/1858) und in der letzten gedruckten Fassung. Textausgabe v. Peter Leyh, Stuttgart/Bad Cannstatt 1977, Beilage: , Erhebung der Ceschichte zum Rang einer Wissenschaft<, S. 451-469, hier S. 461.

30 Droysen, 1977, S. 160.

31 Droysen, 1977, S. 163.

32 Droysen, 1977, S. 159.
} 
Wir hatten ja gesehen, dass es für Droysen im Wesentlichen um zwei Auffassungsweisen geht: Während die Physik auch nur, so Droysen »eine Betrachtungsweise der Natur «33 sei und in der uns umgebenden stets veränderlichen Welt »das im Wechsel Gleiche [...]: die Regel, das Gesetz, den Stoff, die Raumerfüllung « ${ }^{34}$ erkenne, fokussiere die Geschichtswissenschaft auf das >im Gleichen Wechselnde $<$ »daß sich da in der Bewegung immer neue Formen gestalten, so neue und so bedingende Formungen, daß das Stoffliche, an dem sie erschienen als ein sekundäres Moment erscheint, während jede neue Form eine individuell andere ist « $^{35}$. $\mathrm{Zu}$ achten ist hier weniger auf die gewöhnliche Unterscheidung von Stoff und Form, die Droysen hier anführt, als auf die unscheinbarere, aber wirkmächtige Verknüpfung von Neuheit und Individualität. Damit zielt Droysen auf den Punkt, dass wir es in einer Wissenschaft nicht nur mit Anderem, sondern mit Andersartigem, ja auch mit Einzigartigem zu tun haben können. Bei einer solchen Beschaffenheit des Gegenstandes verbietet sich der Rückgang auf Prinzipien, die, sind sie gegeben, stets das Gleiche hervorbringen.

Die nächste Station auf dem Weg der Bipolarisierung von Geistes- und Naturwissenschaften findet sich in Wilhelm Diltheys Einleitung in die Geisteswissenschaften von $1883 .{ }^{36}$ Ausgehend von der »Unmöglichkeit der Ableitung von geistigen Thatsachen aus denen der mechanischen Naturordnung $\ll^{37}$ versucht er die Wissenschaftlichkeit der historischen Wissenschaften philosophisch zu begründen. Das Wort Geisteswissenschaften hält er als Bezeichnung für den Bereich, der vom Menschen geformt und geprägt ist, für provisorisch geeignet, jedenfalls konkurrierenden Begriffen vorzuziehen wie »Gesellschaftswissenschaft (Sociologie), moralische, geschichtliche, Cultur-Wissenschaften $\aleph^{38}$.

Dilthey sah die "Zwecke der Geisteswissenschaften« darin, »das Singulare, Individuale der geschichtlich-gesellschaftlichen Wirklichkeit zu erfassen ${ }^{39}$ durchaus in Bezug auf Gleichförmigkeit, aber Gleichförmigkeit nicht

33 Droysen, 1977, Beilage: >Natur und Geschichte<, S. 470-479, hier S. 477.

34 Droysen, 1977, S. 474.

35 Droysen, 1977, S. 475.

36 Wilhelm Dilthey: Einleitung in die Ceisteswissenschaften. Versuch einer Grundlegung für das Studium der Gesellschaft und der Geschichte. Bd. 1. Leipzig 1883.

37 Dilthey, 1883, S. 12.

38 Dilthey, 1883 , S. 7.

39 Dilthey, 1883 , S. 34. 
im Sinne der Naturwissenschaften, sondern in einem genealogischen Sinne (»wie sie gewachsen sind $\ll)^{40}$

Schärfer begrifflich gefasst hat wenige Jahre später diese Unterscheidung Wilhelm Windelband in seiner Rektoratsrede Geschichte und Naturwissenschaft von 1894. Die Konzeption der Zweiteilung war nicht neu, aber neu war die Terminologie, die Windelband einführte: nomothetische und idiographische Wissenschaft. Schauen wir zunächst auf die Wörter: nomos heißt Gesetz, thesis das Stellen oder Setzen, idios das Einzelne betreffend, eigentümlich, graphein das Schreiben, Beschreiben. Wir können also sagen: gesetzesaufstellend einerseits, eigenheitsbeschreibend andererseits. "Das Einteilungsprinzip ist der formale Charakter der Erkenntnisziele«, schreibt Windelband. »Die einen suchen allgemeine Gesetze, die anderen besondere geschichtliche Tatsachen. $\aleph^{41} » I n$ der einen treibt das Denken von der Feststellung des Besonderen zur Auffassung allgemeiner Beziehungen, in der anderen wird es bei der liebevollen Ausprägung des Besonderen festgehalten. « ${ }^{42}$ Droysens und Diltheys Unterscheidung schimmert hier deutlich durch. Und deutlich zu erkennen ist auch, dass hier die Naturwissenschaften ins Paradigma der episteme eingerückt werden, nämlich als Wissenschaften vom Unveränderlichen und Allgemeinen, während die Geisteswissenschaften im Paradigma des Beschreibens charakterisiert werden. Hier wurzelt der Gemeinplatz, die Naturwissenschaften seien durch "Abstraktion", die Geisteswissenschaften durch »Anschaulichkeit« bestimmt. ${ }^{43}$ Während sich diese letztere (von Kant her rührende) Unterscheidung so bei Droysen nicht findet, schwenkt Windelband in einem anderen, entscheidenden Punkt ganz auf dessen Linie ein: Wie Droysen sieht er den Unterschied der beiden Typen von Wissenschaft nicht als sachlichen, sondern als methodischen; also als einen Unterschied in der Sicht- und Herangehensweise.

Halten wir diesen Punkt fest: Wir haben es an dieser Stelle mit der sehr einfachen und sehr klaren Unterscheidung von zwei Wissenschaftstypen als

40 Herzustellen sei eine »erkenntniß-theoretische Grundlage, die den Thatbestand der Geisteswissenschaften nicht in die Enge einer Erkenntniß von Gleichförmigkeiten nach Analogie der Naturwissenschaft zusammendrängt und solchergestalt verstümmelt, sondern wie sie gewachsen sind, begreift und begründet.«(Dilthey 1883, S. 34.).

41 Wilhelm Windelband: Ceschichte und Naturwissenschaft, Straßburger Rektoratsrede 1894, Straßburg 1904, S. 11.

42 Windelband, 1904, S. 16.

43 Windelband, 1904, S. 17. 
zwei Perspektiven zu tun. Und es wird damit auch klar, dass der hier verhandelte Typ von Naturwissenschaft an sehr spezifische Konstellationen gebunden ist. Denn damit wir sagen können, dass unter den gleichen Bedingungen immer das Gleiche auf die gleiche Weise entsteht, bedarf es kontrollierter Settings. Hierher gehört das Thema der technischen Meisterung und der Laborbedingungen (siehe Kapitel 9 und 10).

\section{Deutungskämpfe II: Kontroversen im 20. Jahrhundert: Einheitswissenschaften, Denkstile, Paradigmen und die Rolle der Wissenschafts- und Technikforschung (STS)}

Mit Droysen, Dilthey und Windelband stoßen wir also im 19. Jahrhundert auf die Absetzbewegung eines beschreibend-interpretierenden Typs der Geistesund Geschichtswissenschaften von einem abstrahierend-erklärenden Paradigma der Naturwissenschaften. Was sich von da an veränderte, war der Ton. Immer schärfer stellten sich die beiden Richtungen in einem »Kampf um den bestimmenden Einfluss auf die allgemeine Welt- und Lebensansicht des Menschen $\aleph^{44}$ gegeneinander. Gehörten die mittleren Jahrzehnte des 19. Jahrhunderts der Profilierung der Geschichts- und Geisteswissenschaften gegenüber den Naturwissenschaften - und zwar in der Weise, dass Idee und Konzept einer solchen Aufteilung überhaupt erst entstand -, setzt nun am Ende des 19. Jahrhunderts eine Art Gegenbewegung an. In verschiedenen Städten gründen sich Zirkel, die eine swissenschaftliche Weltauffassung` propagieren und die Begriffe Positivismus sowie Empirismus erhalten nicht nur neue Vorsilben - Neopositivismus, Logischer Empirismus -, sondern avancieren auch zu Namen für neue Richtungen und Schulen der Wissenschaftstheorie.

Der berühmteste und für die Entwicklung der Wissenschaftstheorie bedeutendste dieser neuen Zusammenschlüsse war der Wiener Kreis. Aus dem Verein Ernst Mach hervorgegangen, veröffentlichte dieser Kreis seit Ende der 1920er Jahren Schriften, die die Spaltung der Wissenschaften aufheben sollten - allerdings unter einer ganz bestimmten Leitlinie: eine "Einheitswissenschaft« im Sinne der `wissenschaftlichen Weltauffassung« sollte nämlich dadurch gekennzeichnet sein, dass alle Erkenntnisse der Einzelwissen- 
schaft sich in einem "neutralen Formelsystem « abbilden lassen. ${ }^{45}$ Ein dazu geeignetes Formelsystem sei durch die moderne symbolische Logik bereitgestellt, die es ermögliche, »den intuitiven Schlussprozeß des gewöhnlichen Denkens zu formalisieren, das heißt in eine strenge, durch den Zeichenmechanismus automatisch kontrollierte Form zu bringen. $\aleph^{46}$ Während man so auf der einen Seite das Denken in formales Schließen überführen wollte ${ }^{47}$ versuchte man aufseiten der Erfahrung jede Beobachtung in $» B e g r i f f e$ eigenpsychischer Erlebnisse « zu überführen, sodass sich ein »Konstitutionssystem der Begriffe« ergibt, das die »Einordnung der Begriffe der verschiedenen Wissenschaftszweige « erlaubt. ${ }^{48}$

Die Grundidee im vom Wiener Kreis geprägten Logischen Empirismus war also, dass die Einheit der Wissenschaften durch eine gemeinsame formale Sprache gewährleistet wird. Dieses Programm gilt zwar inzwischen weithin als gescheitert (entscheidend waren hier u.a. Wittgensteins Überlegungen zu Sprachspielen im Gegensatz zu formalen Sprachen, mit denen er von eigenen früheren Überlegungen, die der Wiener Kreis absorbiert hatte, abrückte) ${ }_{,}^{49}$ aber es führte zu einer einflussreichen Reihe von Schriften und Tagungen unter Titeln wie Einheitswissenschaften, Unified Science und Unity of Science, die bis in die 1960er Jahre reichte. ${ }^{50}$

Dabei verlagerte sich aber mit der Zeit der Grundgedanke. Die Physik stellte zwar auch schon für den Wiener Kreis ein Ideal von Wissenschaft dar, die Vereinigung der Wissenschaften sollte aber nicht im Rahmen einer bestimmten Wissenschaft erfolgen, sondern im Medium einer formalisierten Sprache. Als nun aber 1958 Paul Oppenheim und Hilary Putnam The Unity of Science as a Working Hypothesis veröffentlichen, gehen sie einen Schritt darüber hinaus. Die Einheit der Wissenschaft wird nun darin begründet, dass sich alle wissenschaftlichen Themenbereiche als verschiedene Levels Schritt

45 Verein Ernst Mach (Hrsg.): Wissenschaftliche Weltauffassung. Der Wiener Kreis, Wien 1929, S. 305.

46 Verein Ernst Mach,1929, S. 308.

47 Vgl. Verein Ernst Mach, 1929, S. $306 f$.

48 Verein Ernst Mach, 1929, S. 308.

49 Vgl. Ludwig Wittgenstein: Philosophische Untersuchungen. In: Werkausgabe Bd. 1, Frankfurt a.M. 1982, §23, § 97.

50 Vgl. Friedrich Stadler: Studien zum Wiener Kreis. Ursprung, Entwicklung und Wirkung des Logischen Empirismus im Kontext, Frankfurt a.M. 1997, S. $657 \mathrm{ff}$. 
Abb. 11: Ausschnitt aus Oppenheim/Putnam: The Unity of Science as a Working Hypothesis, S. 9.

(6) The levels must be selected in a way which is "natural" 9 and justifiable from the standpoint of present-day empirical science. In particular, the step from any one of our reductive levels to the next lower level must correspond to what is, scientifically speaking, a crucial step in the trend toward over-all physicalistic reduction.

The accompanying list gives the levels we shall employ; ${ }^{10}$ the reader may verify that the six conditions we have listed are all satisfied.

\begin{tabular}{|c|c|}
\hline & $\begin{array}{l}\text { Social groups } \\
\text { (Multicellular) living things } \\
\text { Cells } \\
\text { Molecules } \\
\text { Atoms } \\
\text { Elementary particles }\end{array}$ \\
\hline
\end{tabular}

für Schritt zurückführen lassen auf den Bereich des Physikalischen (Abb. 11). ${ }^{51}$ »Physicalistic Reduction« nennen das die beiden Autoren (wobei einer, Paul Oppenheim, uns ja bereits als Mitautor des DN-Schemas der Erklärung im Beweisparadigma begegnet ist). Es bedeutet, dass es in den Wissenschaften einen einheitlichen methodologischen Trend gibt zur Übersetzung komplexere Phänomene in kleinere, physikalische Einheiten.

Die physikalistische Sichtweise hat die Wissenschaftsphilosophie über Jahrzehnte beherrscht. Doch so eindeutig lagen auch in dieser Phase, die, grob gesprochen, vom Ende des 19. Jahrhunderts bis zum Ende des 20. Jahrhunderts reichte, die Dinge nicht. Noch Ernst Mach, der vielen als Spiritus rector der szientifischen Bewegung erschien, konnte die physikalische Methodologie anders und vielfältig, nämlich ebenso in den Paradigmen des Beschreibens (siehe Kapitel 14), des Vergleichens (Taxonomie), ${ }^{52}$ des hermeneutischen

51 Paul Oppenheim/Hilary Putnam: The Unity of Science as a Working Hypothesis. In: Minnesota Studies in the Philosophy of Science, 1958, S. 3-35, die graphische Darstellung der »levels«befindet sich aufS. 9.

52 Prinzip der Vergleichung in der Physik: »Wenn es nicht üblich ist, von vergleichender Physik zu sprechen, wie man von vergleichenden Anatomie spricht, so liegt das nur daran, dass bei einer mehr aktiven experimentellen Wissenschaft die Aufmerksamkeit von dem kontemplativen Element allzusehr abgelenkt wird. Die Physik lebt und wächst aber, wie jede andere Wissenschaft, durch die Vergleichung.« Ernst Mach: Die Vergleichung als wissenschaftliches Princip. In: ders. Die Principien der Wärmelehre. Historisch-kritisch entwickelt von Ernst Mach, Leipzig 1896 (3. Aufl. 1919), S. 396-405, hier S. 397. 
Verstehens ${ }^{53}$ und des forscherischen Experimentierens betrachten. ${ }^{54}$ Selbst in den 1920er und 1930er Jahren entstanden noch alternative Konzeptionen. Hier ist z.B. zu nennen der britische Historiker, Philosoph und Archäologe Robin George Collingwood, der in Are History and Science Different Kind of Knowledge 1922 (gemeinsam mit A. E. Taylor und F. C. S. Schiller) eine Wende hin zur wissenschaftlichen Praxis vollzog, die sie ihn unmittelbar in einen hermeneutisch-interpretativen Kontext einrücken ließ. In verschiedenen Denkrichtungen der Zeit (»the schools of Mach, of Bergson, of James, and of Croce«) entdecken die Autoren ein gemeinsames Motiv: »they agree in holding that science is not knowledge at all but action $\ll \cdot{ }^{55}$ Davon ausgehend setzen sie einen Begriff zentral, der die Logik des Wiener Kreises auf den Kopf stellt: Anwendung (»application«). Denn während im Logischen Empirismus Erfahrungssätze in ein formales System integriert werden, an dessen Spitze allgemeine Gesetzesaussagen stehen, sehen sie das wesentliche Moment von Wissenschaft darin, generelle Aussagen auf konkrete Sachverhalte anzuwenden.

»Thus, to be a chemist consists not in knowing general formulae but in interpreting particular changes which we observe taking place by means of these formulae: the science of mechanics consists in the similar interpretation of observed motions: even mathematics does not consist of abstract equations and formulae but in the application of these to the interpretation of our own mathematical operations. ${ }^{56}$

53 Eine Formulierung, die Motive hermeneutischer Adäquation aufnimmt, findet sich in Ernst Mach: Erkenntnis und Irrtum. Skizzen zur Psychologie der Forschung, Leipzig 1905 (2. Aufl. 1906), wo Erkenntnis als » Anpassung der Cedanken an die Tatsachen und aneinander« aufgefasst wird, S. 164-182.

54 Ernst Mach: Über Gedankenexperimente. In ders.: Erkenntnis und Irrtum. Skizzen zur Psychologie der Forschung, Leipzig 1905, S. 183-200. Vgl. dazu auch Birgit Griesecke/ Werner Kogge: Was ist eigentlich ein Cedankenexperiment? Mach, Wittgenstein und der neue Experimentalismus. In: Marcus Krause/Nicolas Pethes (Hrsg.), Literarische Experimentalkulturen. Poetologien des Experiments im 19. Jahrhundert, Würzburg 2005, S. 4172.

55 R. G. Collingwood et al.: Are History and Science Different Kinds of Knowledge?. In: Mind, 124(1922), S. 443-466, hier S. 445.

56 Collingwood et al., 1922, S. 447. 
Es ist bemerkenswert, wie Collingwood (et al.) hier durch eine einzige gedankliche Wendung ein ganz anderes Bild von Wissenschaft hervorbringt: Wenn die formalen Regelsysteme, an deren Spitze die >Naturgesetze< stehen, nicht mehr als Ziel der Wissenschaft, sondern als Mittel gesehen werden, um konkrete Prozesse und Sachverhalte zu deuten, dann wird das Interpretieren zum zentralen Moment von Wissenschaft: "Science is this interpretation«, schreibt Collingwood (et al.), und: »To live the life of a scientist consists in the understanding of the world around one in terms of one's science. $\aleph^{57}$

Eine ganz ähnliche Wendung hin zur Praxis der Wissenschaften vollzog zu dieser Zeit auch der Mikrobiologe und Wissenschaftstheoretiker Ludwik Fleck, dessen 1936 erschienenes Buch Entstehung und Entwicklung einer wissenschaftlichen Tatsache sich explizit mit den Lehren des Wiener Kreises auseinandersetzt. ${ }^{58}$ Flecks Einsatz besteht im Kern darin, dass er anstelle einer Dualität von (formalisierter) Theorie und Empirie das Zusammenspiel von aktiven und passiven Momenten in der Forschung thematisiert. "Jedes Erkennen", schreibt er, »bedeutet zunächst: bei bestimmten aktiv vorgenommenen Voraussetzungen die zwangsmäßig, passiv sich ergebenden Zusammenhänge festzustellen. «9 $^{59}$ Mit »Voraussetzungen« meint Fleck gerade nicht nur explizite Annahmen, sondern das ganze Gewebe der Kenntnisse, der Übung, der Fertigkeiten, der Schulung des Blicks, die ein Wissenschaftler erwirbt, wenn er sich in einem Zweig der Wissenschaft professionalisiert. Fleck nennt dieses disziplinär spezifische und historisch veränderliche Gewebe Denkstil und er ordnet einem Denkstil ein Denkkollektiv zu (wodurch er zu einem der Gewährsleute der Wissenschaftssoziologie wurde).

Neben Ludwik Fleck haben - aus der Philosophie der Sprache heraus Ludwig Wittgenstein (1933) und Gilbert Ryle (1949) die Grundlagen für ein praxisbezogenes Bild von Wissenschaft gelegt. Dass Wissenschaft auch

57 Collingwood et al., 1922, S. 447.

58 So schreibt Fleck: »Es genügt, in der Zeitschrift Erkenntnis Band II (S. 432) und Band III (S. 215) zu vergleichen, um sich davon zu überzeugen, wie sich Carnap mit seinen Protokollen (Protokollsätzen) verwickelt hat, und die völlige Fruchtlosigkeit der ganzen Sache festzustellen.« Ludwik Fleck: Über die wissenschaftliche Beobachtung und die Wahrnehmung im allgemeinen. In: ders.: Erfahrung und Tatsache. Gesammelte Aufsätze. Mit einer Einl. hrsg. v. Lothar Schäfer u. Thomas Schnelle, Frankfurt a.M. 1983 (1935), S. 59- 83, hier S. 64.

59 Ludwik Fleck: Entstehung und Entwicklung einer wissenschaftlichen Tatsache. Einführung in die Lehre vom Denkstil und Denkkollektiv, Frankfurt a.M. 1980, S. 85. 
Könnerschaft ist, die mit praktischer, disziplinärer Schulung, mit der Ausbildung spezifischer Wahrnehmungsfähigkeiten - Stichwort hier: Gestaltsehen - und Einschätzungskompetenz zu tun haben: diesen Gedanken haben Thomas S. Kuhn (1962) und Michael Polanyi (1966) aus den Diskursen der ersten Hälfte des 20 . Jahrhunderts übernommen und damit die weitere Entwicklung der Wissenschaftstheorie maßgeblich beeinflusst. So schreibt etwa Michael Polanyi, der das Konzept eines tacit knowledge ('stilles‘, oder, wie es auch heißt, simplizites Wissen) geprägt hat:

»/ch werde das menschliche Erkennen ausgehend von der Tatsache betrachten, daß wir mehr wissen, als wir zu sagen wissen. [...] - entsprechend der Unterscheidung von $>$ Wissen $<$ und $/ K o ̈ n n e n<$ in der deutschen Sprache oder der Differenz von knowing how und knowing that bei Cilbert Ryle. [... Wenn ich von Wissen spreche, beziehe ich mich also stets sowohl auf praktische als auch auf theoretische Kenntnisse. $\kappa^{60}$

Und Thomas S. Kuhn sekundiert im Postskriptum (1969) zu The Structure of Scientific Revolution:

»Dieses Lernen geht nicht mit ausschließlich verbalen Mitteln vor sich, sondern im Zusammenspiel von gegebenen Formulierungen und konkreten Beispielen für ihren Gebrauch: Natur und Worte werden gemeinsam gelernt. Um es mit Michael Polanyis hilfreicher Formulierung auszudrücken: Das Ergebnis dieses Prozesses ist ein sstillschweigendes Wissen<, das durch die wissenschaftliche Betätigung und nicht durch Aneignung von Regeln dafür erworben wird. « ${ }^{67}$

Kuhn wiederum bekennt in der Einleitung seines wirkmächtigen Werks The Structure of Scientific Revolutions von 1962, dass er entscheidende Anregungen von Ludwik Fleck bezog - und so schließt sich dieser Kreis.

Die Linie von Fleck zu Kuhn steht im Kontext einer Vielzahl von Auseinandersetzungen mit jenem szientistischen Bild von Wissenschaft, das, ausgehend vom Wiener Kreis, die Wissenschaftstheorie des 20. Jahrhunderts

60 Michael Polanyi: Implizites Wissen. Frankfurt a.M. 1985 [1966], S. 14; S. 16.

61 Thomas S. Kuhn: Postskriptum. In: ders.: Die Struktur wissenschaftlicher Revolutionen, Frankfurt a.M. 1988 (1962), S. 186-221, hier S. 203. 
bestimmte. Die These von der tiefen Verbundenheit theoretischen Wissens mit der Praxis, die bedeutet, dass Wissen sich nicht einfach in ein formales System von Aussagen einfügen lässt, war eine erklärte Gegenposition zu diesem dominanten Bild. Sie führte zu einem 'Neuen Experimentalismusর, zu einer Betonung des `Intervening` (eingreifenden Handelns) und zu einem breiten Diskurs darüber, wie die Mittel der Wissenschaft - von Begriffen über Bilder, Diagramme, Modelle und Instrumente bis hin zu Experimentaufbauten - in der Erkenntnis wirksam werden. ${ }^{62}$

Damit sind wir bei dem Gegensatz angelangt, der die Debatten um Wissenschaft seit einiger Zeit am stärksten bestimmt: dem Gegensatz von Konstruktivismus und Realismus.

Konstruktivismus ist ein Streitbegriff - und zwar bereits seit Jahrzehnten. Den einen steht er für eine wissenschaftlich reflektierte und politisch kritische Position, den anderen für das Ende von Wissenschaft und Fortschritt. Diese gegenläufigen Bewertungen können nur sinnvoll diskutiert werden, wenn die Sache klar ist.

Was ist Konstruktivismus? Tatsächlich lassen sich drei Auffassungen unterscheiden, die unter dem Titel Konstruktivismus verhandelt werden. Zwischen diesen dreien gibt es Übergänge und Ähnlichkeiten, aber auch bedeutende Differenzen und Unvereinbarkeiten. Gemeinsam ist ihnen, dass sie Bedingungen von Erkenntnis reflektieren (insofern steht Konstruktivismus im Paradigma der Kritik). Doch diese Bedingungen fassen sie auf dreierlei Weisen als konstruiert auf:

62 Um nur einige Marksteine zu nennen: Nancy Cartwright: How the Laws of Physics Lie, New York 1983; Ian Hacking: Einführung in die Philosophie der Naturwissenschaften, Stuttgart 1996 (im Orig. 1983 (Representing and Intervening)); Holm Tetens: Experimentelle Erfahrung, Hamburg 1987; Don Ihde: Instrumental Realism: The Interface between Philosophy of Science and Philosophy of Technology, Bloomington/Indianapolis 1991; Hans-Jörg Rheinberger: Experiment. Differenz. Schrift: Zur Geschichte epistemischer Dinge, Marburg an der Lahn 1992; Andrew Pickering: The Mangle of Practice: Time, Agency, and Science, Chicago 1995; Joseph Rouse: Engaging Science: How to Understand its Practices Philosophically, Ithaca 1996; Hans-Jörg Rheinberger: Experimentalsysteme und epistemische Dinge. Eine Ceschichte der Proteinsynthese im Reagenzglas, Göttingen 2001. 


\section{BASISWISSEN 13: Konstruktivismus - ein Schlagwort, unterschiedliche Bedeutungen}

\begin{tabular}{|c|c|c|c|}
\hline & $\begin{array}{l}\text { (I.) konventionalis- } \\
\text { tischer Konstrukti- } \\
\text { vismus }\end{array}$ & $\begin{array}{l}\text { (II.) erkenntnistheo- } \\
\text { retischer Konstrukti- } \\
\text { vismus }\end{array}$ & $\begin{array}{l}\text { (III.) wahrnehmungs- } \\
\text { theoretischer, biolo- } \\
\text { gisch-systemischer } \\
\text { Konstruktivismus }\end{array}$ \\
\hline Grundthese & $\begin{array}{l}\text { Konzepte (wie z.B. Gerechtig- } \\
\text { keit; Nation; Fremdheit; } \\
\text { Geschlecht; Natur; Gen) sind } \\
\text { als Produkte einer sozialen } \\
\text { Gemeinschaf in ihren jeweili- } \\
\text { gen, historisch konstituierten } \\
\text { Konventionen und aus ihren } \\
\text { sprachlich, diskursiv und } \\
\text { praktisch manifestierten } \\
\text { Handlungsmustern zu ver- } \\
\text { stehen }\end{array}$ & $\begin{array}{l}\text { Erkenntnis ist bestimmt durch } \\
\text { die menschlichen Erkennt- } \\
\text { nismittel und -vermögen. } \\
\text { Die Struktur der Vernunft } \\
\text { bestimmt, wie Gegenstände } \\
\text { konzeptuell - auch wissen- } \\
\text { schaftlich - erfasst werden }\end{array}$ & $\begin{array}{l}\text { Ein System (Organismus; } \\
\text { Zentrales-Nerven-System; } \\
\text { Gehirn; Kultursystem) nimmt } \\
\text { die Außenwelt nach Maßgabe } \\
\text { ihrer Binnenstruktur wahr und } \\
\text { projiziert >Realität‘ als Pro- } \\
\text { dukt innersystemischer und } \\
\text { evolutionärer Anforderungen } \\
\text { nach außen }\end{array}$ \\
\hline $\begin{array}{l}\text { Grund- } \\
\text { motiv }\end{array}$ & $\begin{array}{l}\text { Entdeckung der Rolle der } \\
\text { Konventionen (Gewohnheiten; } \\
\text { Gebräuche, soziale Muster) in } \\
\text { / anstatt zeitloser Ordnung } \\
\text { (Kosmologie) }\end{array}$ & $\begin{array}{l}\text { Priorisierung des Rationalen } \\
\text { gegenüber der unmittelbaren } \\
\text { Erfahrbarkeit von Wirklichkeit }\end{array}$ & $\begin{array}{l}\text { Radikalisierung des } \\
\text { Perspektivismus }\end{array}$ \\
\hline $\begin{array}{l}\text { historische } \\
\text { Quellen } \\
\text { für diese } \\
\text { Spiel- } \\
\text { art von } \\
\text { Konstrukti- } \\
\text { vismus }\end{array}$ & $\begin{array}{l}\text { antiker Konventionalismus: } \\
\text { Sophistik; diskutiert z.B. in } \\
\text { Platons Kratylos; } \\
\text { Marx; Dilthey } \\
\text { Wissenssoziologie }\end{array}$ & $\begin{array}{l}\text { Kants Kritik der reinen Ver- } \\
\text { nunft mit ihrer 'kopernikani- } \\
\text { schen Wender; } \\
\text { Der transzendental-philoso- } \\
\text { phische Ansatz hat sich im } 20 . \\
\text { Jahrhundert als logizistische } \\
\text { Variante ausgeprägt }\end{array}$ & $\begin{array}{l}\text { Descartes Wahr-nehmungs- } \\
\text { theorie } \\
\text { Nietzsches biologistischer } \\
\text { Perspektivismus } \\
\text { Kybernetik }\end{array}$ \\
\hline $\begin{array}{l}\text { moderne } \\
\text { Klassiker }\end{array}$ & $\begin{array}{l}\text { Fleck 1936: Entstehung und } \\
\text { Entwicklung einer wissen- } \\
\text { schaftlichen Tatsache } \\
\text { Wittgenstein 1953: Philosophi- } \\
\text { sche Untersuchungen } \\
\text { Foucault 1966: Les Mots } \\
\text { et les choses } \\
\text { Latour/ Woolgar 1979: Labora- } \\
\text { tory Life. } \\
\text { The Construction } \\
\text { of Scientific Facts }\end{array}$ & $\begin{array}{l}\text { Russell 1918: The Philosophy of } \\
\text { logical Atomism } \\
\text { Hilbert 1918: Axiomatisches } \\
\text { Denken } \\
\text { Carnap 1928: Der logische } \\
\text { Aufbau der Welt } \\
\text { Goodman 1978: } \\
\text { Ways of Worldmaking }\end{array}$ & $\begin{array}{l}\text { Uexküll 1909: Umwelt und } \\
\text { Innenwelt der Tiere } \\
\text { Maturana/ Varela 1980: Auto- } \\
\text { poiesis and Cognition } \\
\text { Schmidt (Hrsg.) 1987: Der } \\
\text { Diskurs des Radikalen Konst- } \\
\text { ruktivismus } \\
\text { Roth 1994: Das Gehirn und } \\
\text { seine Wirklichkeit }\end{array}$ \\
\hline
\end{tabular}




\section{VERTIEFEND Spielarten des Konstruktivismus}

Sieht man auf die Grundmotive aller drei Spielarten von Konstruktivismus, dann zeigt sich als gemeinsamer Nenner eine bestimmte Wendung: Es ist keine objektive, zeitlose und universale Wirklichkeit, die bestimmt, wie eine konzeptuelle Ordnung, wie Erkenntnis oder Wahrnehmung in sich gegliedert und organisiert ist. Konstruktivismen haben allgemein etwas damit zu tun, dass im Erfassen von Wirklichkeit die Bedingungen und Voraussetzungen dieses Erfassens - modern gesprochen: das subjektive Moment - zur Geltung gebracht wird. Doch bereits die jeweilige Grundthese zeigt, dass für die drei Spielarten von Konstruktivismus drei sehr verschiedene Instanzen an die Stelle einer bestimmenden Wirklichkeit rücken:

1. Konventionalistischer Konstruktivismus: An die Stelle einer objektiven, zeitlosen und universalen Wirklichkeit treten als Bestimmungsinstanz soziale Regelungen und Bedeutungszuschreibungen bis hin zu sozialen Strukturfaktoren wie Geschlecht, Schicht, Institution, die - selbst Produkt sozialer Prozesse - wiederum konstitutiv für (soziale) Tatsachen sind. Für diese Spielart ist die These einer radikalen Konventionalität sprachlicher Bedeutung (vs. Natürlichkeit) - wie sie bereits in Platons Kratylos verhandelt wird - zentral, denn sie erst verschafft den Freiraum gegenüber essentialistischen Vorstellungen, die Konzepte an das Wesen der Sachen binden.

2. Erkenntnistheoretischer Konstruktivismus: An die Stelle einer Wirklichkeit, die sich in der Erkenntnis unmittelbar ausdrückt, tritt die Struktur der erkennenden Instanz - bei Kant die Struktur der Erkenntnisvermögen (Kritik der reinen Vernunft). Für einen erkenntnistheoretischen Konstruktivismus ist diese Struktur allerdings nicht schlicht gegeben, sondern bedarf einer eigenen Rekonstruktion rationaler Bedingungen, weshalb das Kantische Projekt als Rekonstruktion der logisch-mathematischen Weltkonstitution fortgeführt werden konnte - etwa bei Russell, beim frühen Wittgenstein, bei Hilbert, Carnap und Goodman.

3. Wahrnehmungstheoretischer, biologisch-systemischer Konstruktivismus: Die Entdeckung der Vermitteltheit von Wirklichkeit in der Wahrnehmung durch Transformation in Nervenreize, die bereits Descartes anführt, in Verein mit physiologischen Selbstorganisations-Konzepten, die sich im 18. Jahrhundert, aber auch bereits bei Aristoteles finden, sorgen dafür, 
dass an die Stelle einer objektiven Wirklichkeit je spezifische biologische - oder auch psychologische, allgemein: systemlogische - Bestimmungsinstanzen treten.

Vergleicht man die drei Ersetzungen einer objektiven, zeitlosen und universalen Wirklichkeit - nämlich soziale Konventionen, rationale Strukturen und systemische Konstitution - miteinander, so wird offenkundig, dass hier Inkompatibilitäten auftreten. Offensichtlich sind z.B. mit sozialen Konventionen weder Strukturen von Rationalität gemeint, noch (naturgegebene) systemische Konstitutionen.

Es sind drei Implikationen, die dem Konstruktivismus häufig zugeschrieben werden, an denen sich weitere Unvereinbarkeiten zeigen:

1. Die Kontingenzthese: Das entscheidende Moment konstruktivistischer Theorieansätze liegt für Viele darin, dass `scheinbar` naturgegebene, nur naturwissenschaftlich zugängliche Gegenstände in den Bereich sinnhafter Konzepte und sozialer Aushandlung rücken. Sie sind nicht notwendig so wie sie erscheinen, sondern auf einem der vielfach möglichen Wege entstanden und können prinzipiell anders sein. Naturalisierung und Substantialisierung zeigen sich in dieser Perspektive als ideologische Strategien, die sich konzeptpolitisch unterlaufen lassen. Die Kontingenzthese wird allerdings nur durch die konventionalistische Variante (I) unterstützt, während die erkenntnistheoretische Variante (II) in Subjektivität (resp. Logik) eine sogar gesteigerte universale Ordnung zugrunde legt und auch die systemische Variante (III) die konstitutiven Strukturen wiederum objektiviert. Sozialisierung und Semantisierung des Gegebenen ist also das Anliegen nur einer Variante von Konstruktivismus, nämlich der konventionalistischen (I).

2. Perspektivismus/Relativismus: Eine viel diskutierte Zuschreibung an konstruktivistische Theorieansätze ist die der Perspektivierung und Relativierung von Konzeptionen, Erkenntnis und Wahrnehmung. Im Sinne der allgemeinen Annahme, dass es keine >unbedingte Wirklichkeit gibt, trifft dies auch tatsächlich auf alle Spielarten von Konstruktivismus zu. Eine stärkere These, die individuelle, autonome und geschlossene Konstitutionssysteme annimmt - Stichwort >Solipsismus $<$-, vertritt allerdings nur die dritte Spielart (III). Gänzlich im Gegensatz zu dessen 
auf die Perspektive von Einzelsystemen bezogenen partikularistischen Konstruktivismus steht ein erkenntnistheoretischer Konstruktivismus (II), der ja gerade universale Bedingungen von Erkenntnis rekonstruiert. Und auch ein sozialer Konstruktivismus (I) ist nicht auf einen partikularistischen Relativismus festgelegt, da ja Sinnsysteme und Sinnprozesse immer ein Potenzial zu ihrer Überschreitung implizieren. Nur ein radikaler Konstruktivismus der systemischen Spielart (III) führt zu einer solipsistischen Position.

3. Ausblendung von Rezeptivität: Die erkenntnistheoretische (II) und die systemische Variante (III.) von Konstruktivismus tendieren dazu, Wahrnehmung und Erkenntnis komplett als `Leistung des Subjekts zu konzipieren. Ein >Gewordensein<, eine Prägung und Ausbildung der subjektiven Strukturen aus Geschichte und Interaktion, werden hier theoretisch ausgeblendet oder negiert. Auch manche konventional-konstruktivistischen Ansätze, wie z.B. der Sprachrelativismus nach der Sapir-Whorf-Hypothese, tendieren ebenfalls zu einer Festlegung auf gegebene Strukturen in Absehung von ihrer Entstehung. Genealogische Einsätze finden sich z.B. bei Foucault (neben seinem methodischen Beharren synchroner Schnitte) und bei Thomas S. Kuhn. Die Frage hier ist, inwiefern die Autonomie der sozialen Systeme diese von allem, was nicht bereits konventional durchdrungen ist, entkoppelt. Derzeit aktuelle Themen der Theoriebildung wie Experimentalismus, Materialität, Verkörperung, Alterität und Responsivität zeigen an, dass In-der-Welt-sein mehr heißen kann als ein Kreisen in eigenen Konventionen und Strukturen, selbst wenn ein unmittelbarer Rekurs aufWirklichkeit wohl keine theoretische Zukunft mehr hat.

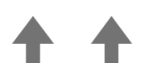

Wir haben bereits davon gesprochen, wie leicht man sich in den unterschiedlichen Fäden dieser Begriffe verheddern kann. Will man auf den Punkt bringen, was der Gegensatz von Konstruktivismus und Realismus für unser Bild von Wissenschaft bedeutet, dann geht es letztlich um eines: Kann wissenschaftliches Wissen in sich selbst begründet sein, oder ist es durch bestimmte Faktoren bedingt und je perspektiviert (wie alles andere Wissen auch)? Es scheint viel auf dem Spiel zu stehen bei dieser Frage: Auf der einen Seite steht sämtliches Bemühen um Strenge, Prüfung, Nachweis, Neutralität, Objektivität usw., die das wissenschaftliche Arbeiten idealerweise bestimmen; 
auf der anderen Seite steht die Ablehnung einer jeglicher Auffassungen, die behaupten, sich in sich selbst begründen zu können und die Autorität der Wahrheit - zumindest des bestbegründetsten Wissens - für sich beanspruchen zu können. Diese Grundmotive kommen aber kaum zur Sprache. Stattdessen wird von der einen Seite auf Erkenntnisfortschritt hin zur Realität insistiert, während die Gegenseite gebetsmühlenhaft wiederholt, die Wissenschaft konstruiere ihre Konzepte und Erkenntnisse. Während die eine Seite gegen Relativismus angeht, kämpft die andere gegen alle Formen von Letztbegründung.

Forschende Wissenschaftler haben mit diesen Gegensätzen für gewöhnlich wenig am Hut. Einerseits erschiene es ihnen absurd, ihre Ergebnisse konstruiert zu nennen (es kommt ihnen ja gerade darauf an, dass sie sich sergeben`), andererseits gehört zu ihrer konkreten Erfahrung stets die der Gebundenheit und begrenzten Reichweite ihrer Ergebnisse - die stets nur Zwischenergebnisse sind. Der Konflikt wird mehr auf weltanschaulicher, wissenschaftspolitischer - und natürlich auch auf der Ebene des Kampfes um Mittel und Ressourcen ausgetragen.

Eine besondere Rolle in diesem Konflikt spielen Wissenschaftssoziologie und Wissenschaftsgeschichte, die sich im 20. Jahrhundert allmählich ausbildeten und heute unter dem Titel Science and Technology Studies (STS) einen international vernetzten Diskurs bilden. ${ }^{63}$ Wenn wir die Debatten um Konstruktivismus in den Wissenschaften verstehen wollen, müssen wir eine Besonderheit berücksichtigen, die in der Rolle von STS liegt: Wissenschaftsforschung ist einerseits selbst eine Wissenschaft, andererseits ist ihr Gegenstand Wissenschaft. Diese Konstellation stellte so lange kein besonderes Problem dar, wie die Wissenschaftsforschung die Wissenschaften nur unter äußeren Gesichtspunkten betrachtete. Als aber der Gegenstand der Wissenschaften nicht mehr nur die historischen und sozialen Gegebenheiten in den Wissenschaften waren, sondern sich mehr und mehr auf die Frage verlagerte, wie Wissenschaften Wissen produzieren und weiterhin, wovon es abhängt, was als Wissen gilt, da entstand eine neue Konkurrenzsituation: Wer hat welche Deutungsbefugnis über wissenschaftliches Wissen: Diejenigen, die es produzieren oder diejenigen, die die Produktion beobachten?

63 Vgl. Ulrike Felt/Helga Nowotny/Klaus Taschwer: Wissenschaftsforschung. Eine Einführung, Frankfurt a.M. u.a. 1995, S. 22-28. 


\section{VERTIEFEND Wissenschaftliches Wissen in der Wissenschaftssoziologie}

Die Geschichte der Wissenschaftsforschung lässt sich kurz so umreißen: Vor dem Hintergrund von Karl Marx' Diktum, das Sein bestimme das Bewusstsein, entwickelte sich Anfang des 20. Jahrhunderts eine Wissenschaftssoziologie (bekanntester Vertreter: Karl Mannheim), die »[m]enschliches Denken und Wissen [...] systematisch mit den sie bestimmenden sozialen Faktoren - wie Religion, Klasse etc. - in Beziehung ${ }^{64}$ setzte. Das heißt, es wurde eine für die Soziologie grundständige Operation vorgenommen: Bestimmte soziale Gegebenheiten (wie z.B. Wahlverhalten, Überzeugungen, Normen) werden korreliert mit sozialen Strukturen. Dabei nahm Mannheim von dieser unproblematischen Anwendung soziologischer Verfahren auf die Wissenschaften Mathematik und Naturwissenschaften explizit aus.

Die frühe Wissenschaftssoziologie, für die der US-amerikanische Soziologe Robert K. Merton prägend wirkte, folgte dem nicht. Allerdings beschränkte sie sich hinsichtlich ihres Untersuchungsgegenstandes auf andere Weise: Sie befasste sich ausschließlich mit den der Erkenntnis äußerlichen, sozialen Faktoren, also auf den Aspekt der Wissenschaft als Institution:

"Wir wollen uns hier zunächst auf die kulturelle Struktur der Wissenschaft, das heißt, auf einen ganz bestimmten Aspekt der Wissenschaft als Institution beschränken. Es geht hier also nicht um die Methoden der Wissenschaft als solche, sondern um die Verhaltensmaßregeln, in die diese Methoden eingebettet sind. ${ }^{65}$

Als soziales Gebilde und Institution kann Wissenschaft genauso untersucht werden wie jeder andere soziale Zusammenhang auch. Man kann ihre Organisationsformen, ihre Gruppenbildung, ihre Kommunikationsregeln, ihre Normen und ihre Mechanismen der Bewertung und Belohnung untersuchen - und zwar kann man dies in systematischer wie auch in historischer Perspektive tun.

64 Felt, Nowotny, Taschwer, 1995, S. 122.

65 Robert K. Merton: Die normative Struktur von Wissenschaft. In: ders.: Entwicklung und Wandel von Forschungsinteressen. Aufsätze zur Wissenschaftssoziologie, Frankfurt a.M. 1995 (1942), S. 86-99, hier S. 88. 
Entscheidend anders stellt sich die Sache dar, wenn es nicht mehr um Wissenschaft als soziales Gebilde, sondern um wissenschaftliches Wissen geht; wenn nicht mehr gefragt wird, wodurch bestimmte Verhaltensformen bedingt sind, sondern wodurch bestimmtes Wissen bedingt ist. Genau diese Wende bestimmte die Wissenschaftsforschung seit den 1960er Jahren. Ausschlaggebend war eine bestimmte Konvergenz: die Wissenschaftssoziologie verband sich nun nämlich mit der Kritik, die sich innerhalb der Wissenschaftsphilosophie am Wissenschaftsbild des Logischen Empirismus entwickelte. Von Karl Popper, der die zugrundeliegende Weltauffassung noch teilte, bis hin zu Thomas S. Kuhn und Paul Feyerabend wurde dieses Bild immer schärfer angegriffen und infrage gestellt: Wissenschaftliches Wissen schien sich nun nicht mehr in einem einheitlichen logischen Aussagesystem abbilden zu lassen, sondern erwies sich als eingebettet in unterschiedliche Methoden und Forschungsparadigmen.

Damit deutete sich eine innere Korrelation an - genauer: die Differenz zwischen innerer und äußerer Bedingtheit fiel in sich zusammen. Denn wenn wissenschaftliches Wissen stets nur unter jeweils speziellen und voraussetzungsreichen Umständen zustande kommt, dann gehören zu diesen Bedingungen auch die sozialen Bedingungen, und zwar insbesondere die Kompetenzen, die praktische und die theoretische Schulung, die Voraussetzung und Ausbildung von Überzeugungen, kurz: alles das, was diejenigen Wissenschaftler, die zur Wissensbildung in einem Bereich beitragen, miteinander verbindet und ihre Zusammenarbeit ermöglicht. Genau hier ist der Einsatz von Ludwik Flecks Lehre vom Denkstil und Denkkollektiv, das die Wissenschaftsforschung - in einer Art Nachwirkung von Kuhns Referenz seit den 1990er Jahren mehr und mehr prägte.

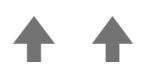

Die Auseinandersetzungen in dieser Frage gipfelten schließlich im sogenannten Science War. Die speziellen Ereignisse dieser Debatte mit fingierten Publikationen, die beweisen sollten, dass im postmodernen Diskurs jeder `Elegante Unsinn ${ }^{66}$ publikationswürdig erscheint (was aber auch in Gegenrichtung ge-

66 Der polemische Titel steht im Kontext der sSokal-Affäre Sokal/Jean Bricmont: Eleganter Unsinn. Wie die Denker der Postmoderne die Wissenschaften mißbrauchen, München 1999. 
lang), können wir hier beiseitelassen. Wichtig ist das Grundmotiv dieser neuen Ausprägung des Zwei-Kulturen Streits (siehe Kapitel 4). Letztlich geht es darum, ob Wissenschaft - und zwar ist das Modell hier stets die Physik - mit sozialen und kulturellen Bedingungen so tief verbunden ist, dass auch wissenschaftliches Wissen stets im Zusammenhang mit seinen sozialen und kulturellen Bedingungen zu verstehen ist; oder aber: ob nicht Wissenschaft in sich selbst über Wahrheits- oder Objektivitätskriterien verfügt, die es von solchen Bedingungen abkoppelt. Dieser Gegensatz ist mit weitreichenden Autoritätsfragen verbunden: Auf der einen Seite steht die Reichweite der Aussagen von Wissenschaftssoziologie, Wissenschaftsgeschichte, ja: allgemein der kulturwissenschaftlichen Fächer auf dem Spiel; auf der anderen Seite die Reichweite der Aussagen über Natur. Oberflächlich betrachtet kann es so scheinen, als ob nur von kulturwissenschaftlicher Seite her die naturwissenschaftliche Objektivität angegriffen werde, wogegen diese verteidigt wurde. Aber tatsächlich richtet sich der Angriff ebenso in umgekehrte Richtung: Für die historischen und soziologischen Fächer gehört es ja - wie wir sahen - zum Kerngeschäft, die Bedingungen historischer und sozialer Begebenheiten zu analysieren und ihr Entstehen zu rekonstruieren. Würde ein Bereich, der die modernen Gesellschaften offenbar tiefgreifend prägt, nämlich die Wissenschaft, aus dem Gegenstandskorpus herausgenommen, dann wären viele soziale Phänomene nicht mehr angemessen beschreibbar. Diese Disziplinen müssen deshalb davon ausgehen, dass auch wissenschaftliches Wissen und Handeln durch je spezifische Bedingungen geprägt sind, wie jedes andere soziale Wissen und Handeln auch. Das bedeutet nicht zugleich, dass der Wert wissenschaftlichen Wissens infrage gestellt wird; es bedeutet lediglich, dass es keine überzeitlichen und in allen Zusammenhängen gültige Wertkriterien gibt.

Aus der Perspektive des Paradigmas der Kritik, zu dem die Wissenschaftsforschung gehört (betrachtet man ihre tiefsten Motive), entsteht ein Bild von Wissenschaft, die sich ihrer eigenen Bedingtheit bewusst ist, diese reflektiert und sich gerade dadurch weiterentwickelt. Und dieses Bild ist sehr wohl vereinbar mit dem Selbstverständnis vieler Forscherinnen und Forscher. Den Wissenschaftstypus der Kritik in diesem Sinne auszubuchstabieren und zu stärken könnte deshalb auch einen guten Weg aus den Kämpfen um wissenschaftliche Deutungshoheit weisen.

Was die Wissenschaftsforschung erstaunlicherweise wenig praktizierte, war die Untersuchung unterschiedlicher Formen und Typen von Wissenschaft. 


\section{Einteilungen von Wissenschaften II: Wissenschaftskulturen werden Thema}

Die Wissenschaftstheorie des 20. Jahrhunderts wurde mehr von theoretischen Leitbegriffen als von Beschreibungen unterschiedlicher Wissenschaften geprägt. Realismus, Konventionalismus, Instrumentalismus, Konstruktivismus sind Schlagworte für Positionen, die die Rollen von Theorie und von Empirie in je unterschiedlichen Auffassungen ausbuchstabieren. Doch von diesen Positionen aus liefert man sich eher einen Streit um Wissenschaft und Wissenschaftlichkeit als einen Einblick in tatsächliche wissenschaftliche Praxis zu gewähren. Zudem orientierten sich diese Debatten so eng am Leitparadigma der mathematisierten Physik, dass andere Traditionen wie die naturgeschichtlichen und hermeneutischen ausgeblendet wurden.

Immerhin in den Blick genommen haben solche Unterschiede einige Ansätze, die sich am Rande des Hauptstroms der Wissenschaftstheorie angesiedelt haben.

Da wäre zum einen ein Ansatz aus den 1950er Jahren, der uns schon begegnet ist, nämlich Stephen Toulmins Einteilung in Wissenschaft vom Typ Physik und Wissenschaft vom Typ Naturgeschichte. Toulmin versucht in immer neuen Anläufen »den Unterschied zwischen erklärenden Wissenschaften wie der Physik und den beobachtenden, sammelnden und beschreibenden Wissenschaften $\mathrm{zu}$ verstehen, die man unter der - schon etwas altertümlich gewordenen - Bezeichnung >Naturgeschichte zusammenfassen könnte « ${ }^{67}$. Für den Typus Physik sieht Toulmin, wie oben bereits deutlich wurde (siehe Kapitel 8), sehr klar, dass in ihr tatsächlich keine Beweisverfahren vom Typ Syllogismos zum Einsatz kommen, sondern Folgerungstechniken gefunden werden, mit denen ein ganzer Phänomenbereich regelhaft behandelt werden kann. So kann man z.B. »unter Verwendung der Techniken der geometrischen Optik« die Länge eines Schattens unter gegebenen Bedingungen »erschließen ${ }^{68}{ }^{68}$ Diese Techniken sind konstruktive Techniken, keine logischen Ableitungen und "genau dies«, schreibt Toulmin, »ist der Grund, weshalb man bedauern muß, dass viele Logiker sich ange-

67 Stephen I. Toulmin: Einführung in die Philosophie der Wissenschaft, Göttingen 1969 (im Orig. 1953), S. 44.

68 Toulmin, 1969, S. 33. 
wöhnt haben, bei wissenschaftlichen Entdeckungen von sinduktivem Schließen zu sprechen: denn wo überhaupt keine Schlußregel angegeben werden kann, wird der Begriff des Schließens gegenstandslos. « ${ }^{69}$

Während Toulmin so die Wissenschaft vom Typ Physik aus den weit reichenden Fängen der Beweislehre befreit, schreibt er diese Form der Schlussfolgerung aber gerade der Naturgeschichte zu: »[W]enn der Physiker in der Lage ist, sein Problem zu formulieren, sieht es nicht so aus wie die Probleme von Botanikern und Zoologen - d.h. nicht so, als ob es ihm nur darauf ankäme, allgemeine Aussagen über salle Felsblöcke` oder salle Flammen` zu machen, wie es der Fall wäre, wenn es sich bei der Physik um die Naturgeschichte der unbelebten Materie handelte. $\aleph^{70}$ Dass dieses Bild von deskriptiven Wissenschaften zu einseitig ist, haben wir in Kapitel 7 gesehen.

Eine andere Einteilung nimmt der italienische Historiker und Kulturwissenschaftler Carlo Ginzburg vor (einiges dazu schon in Kapitel 12). In seinem im Jahr 1979 publizierten Text Spie. Radici di un paradigma indiziaro unterscheidet er ein Indizienparadigma von der Galileischen Wissenschaftsauffassung und charakterisiert das Indizienparadigma wie folgt:

»Die Fähigkeit, ein gebrechliches Pferd am Fersengelenk, ein kommendes Gewitter durch eine plötzliche Veränderung des Windes oder eine feindselige Absicht in der Verfinsterung der Gesichtszüge zu erkennen, wurden natürlich nicht in die Abhandlungen über Pferdezucht, Meteorologie oder Psychologie übernommen. Auf jeden Fall waren diese Formen von Wissen reicher als irgendeine schriftliche Kodifizierung; sie wurden nicht Büchern, sondern der lebendigen Stimme, den Cesten und den Blicken entnommen; sie gründeten sich auf scharfsinnige Beobachtung, die natürlich nicht formalisierbar und oft nicht einmal in Worte übersetzbar waren; sie konstituierten ein teils einheitliches, teils zerstreutes Bildungsgut von Männern und Frauen aller Klassen. Eine subtile Verwandtschaft vereinte sie; alle entstanden aus der Erfahrung, aus der Konkretheit der Erfahrung. Darin bestand die Stärke dieses Typs von Wissen und seine Schwäche bestand in der Unfähigkeit, sich der mächtigen und schrecklichen Waffe der Abstraktion zu bedienen. ${ }^{71}$

69 Toulmin, 1969, S. 43.

70 Toulmin, 1969, S. $114 \mathrm{f}$.

71 Cinzburg, 1988, S. 104. 
Der Zugriff der Abstraktion zeichnet für Ginzburg die Galileische Wissenschaftsform aus. Diese Form setzt voraus, dass die mathematische Sprache einen Zugang zu den relevanten Gegenständen der Forschung bereitstellt, gerade indem sie diesen Gegenstand abstrakt fasst, nicht in seiner konkreten Individualität. Das führt Ginzburg zu dem Schluss:

»An diesem Punkt eröffnen sich zwei Möglichkeiten: entweder man opfert die Erkenntnis des individuellen Elementes zugunsten der (mehr oder weniger streng mathematisch formulierbaren) Verallgemeinerung, oder man versucht - sich langsam vortastend - ein anderes Paradigma zu erarbeiten, das sich auf die wissenschaftliche Erkenntnis des Individuellen stützt (wobei es sich um eine Wissenschaftlichkeit handelt, die völlig neu zu definieren wäre). $\ll^{72}$

Was Ginzburg nicht sieht: er erfindet in schöner Formulierung gleichsam das Rad neu, denn nichts anderes hatte - wie wir bereits sahen - die Hermeneutik des 19. Jahrhunderts seit Droysen bereits ausgeführt. Und wie diese kommt auch Ginzburg dazu, die Gegenüberstellung als eine der Sicht- und Vorgehensweise anzusehen. So zeigt er in sich seinem jüngeren, 2007 erschienenen Text Spuren einer Paradigmengabelung: Machiavelli, Galilei und die Zensur der Gegenreformation verunsichert, wie tief der Gegensatz sich tatsächlich in unterschiedlichen wissenschaftlichen Disziplinen abbildet. Eine Anmerkung Italo Calvinos, dass auch Galileis »Betrachtungsweise der Position der Erde im Sonnensystem [...] ein Interesse für eine Singularität« dokumentiere und der Hinweis von Tullio Regge, »dass auch Physiker mit Indizien, Spuren, nebensächlichen Details usw. arbeiten ${ }^{73}{ }^{73}$ warf für Ginzburg die Frage auf, ob es sich bei der Gegenüberstellung der Paradigmen nicht lediglich um Modi von kognitiver Einstellung handele - und nicht um disziplinäre Konstellationen.

Wir sehen also: Immer wieder kommt im 20. Jahrhundert die Dualität von zwei Typen von Wissenschaft in den Blick; dabei erscheinen durchaus

72 Ginzburg, 1988, S. 108.

73 Carlo Ginzburg: Spuren einer Paradigmengabelung: Machiavelli, Galilei und die Zensur der Gegenreformation. In: Sybille Krämer/Werner Kogge/Gernot Grube (Hrsg.): Spur. Spurenlesen als Orientierungstechnik und Wissenskunst, Frankfurt a.M. 2007, S. 257-280, hier S. 258. 
neue Varianten wissenschaftlicher Praxis auf dem Schirm - wie die der Symboltechniken und die des Spurenlesens. Doch: Die Komparatistik steht jeweils auf schwachen Beinen und Abgrenzungen tun dem Gegenüber oft unrecht. Das scheint, auf den ersten Blick, anders gelagert in einem Fall, dem wir uns jetzt etwas ausführlicher zuwenden, da sich an ihm einige Aspekte der Problematik exemplarisch aufweisen lassen:

Der Zoologe und Wissenschaftshistoriker Alistair Cameron Crombie, der sich in den 1950er und 6oer Jahren intensiv mit Wissenschaft an der Schnittstelle zwischen Spätmittelalter und Frühmoderne beschäftigte, hat eine breit angelegte Studie zur Einteilung von Wissenschaftsarten vorgelegt. Von dieser Epoche ausgehend erweiterte er nach und nach das Spektrum, bis er in der abendländischen Geschichte insgesamt sechs unterschiedliche Scientific Thought Styles ausmachte.

Crombies Ansatz scheint zunächst einer Darstellung unterschiedlicher Wissenschaftstypen, wie wir sie hier versucht haben, eng verwandt zu sein. Allerdings: Der Schein trügt hier. Zwar unterscheidet Crombie sechs Stile von Wissenschaft, unter anderem solche, die auch hier aufgeschlüsselt wurden, aber bei genauerer Betrachtung schlägt er sie allesamt über den Leisten eines einzigen Typus: den der Beweislehre (apodeixis/demonstratio). Er erzeugt so ein Bild der Wissenschaftsgeschichte als einer Entwicklung, die sich zwar verzweigt, aber doch, so könnte man sagen, immer am selben Stamm entlang wächst.

Die wissenschaftlichen Stile, die Crombie in der Geschichte des Denkens ausfindig macht, sind:

»(1) postulation [mathematisches und logisches Beweisen],

(2) the experimental argument, to control postulation and to explore complex subject-matters, and

(3) hypothetical modeling, all concerning individual regularities, and

(4) taxonomy,

(5) probabilistic and statistical analysis, and

(6) historical derivation, all concerning regularities of populations in space and time ${ }^{74}$

74 Alistair Cameron Crombie: Styles of Scientific Thinking in the European Tradition. A history of argument and explanation especially in the mathematical and biomedical sciences and arts, Bd. 1 , 
Diese sechs Modi dekliniert Crombie sowohl in seinem dreibändigen Werk Styles of Scientific Thinking in the European Tradition als auch in weiteren Texten durch. ${ }^{75}$ Neben Beweislehre und experimenteller Wissenschaft finden wir hier die taxonomischen Wissenschaften der Naturgeschichte, die Statistik und einen Ansatz der Geschichtswissenschaften wieder.

Nun ist es aber symptomatisch, dass Crombie von Stilen spricht, nicht von Formen, Arten oder Typen. ${ }^{76}$ In diesem Wort liegt, dass es sich um eher äußerliche Unterschiede, nicht um markante Eigenformen handelt. Und genau so legt Crombie seine historische Entfaltung von Wissenschaftsstilen auch an, wenn er schreibt:

»When we speak today of natural science, we mean a specific style of rationality created within European culture [....] European Rationality is a style of thinking that may be traced to the ancient Greek commitment to this mode of control and decision [...] The Greeks introduced an exclusive form of rationality based on two fundamental ideas: universal, self-consistent and discoverable natural causality and, matching this, formal proof. ${ }^{77}$

Crombies Wissenschaftsstile unterscheiden sich durchaus voneinander, in ihren Objekten, ihren Weisen der Evidenzbildung, und in ihren Kriterien für wissenschaftliche Ergebnisse. ${ }^{78}$ Doch sie stehen allesamt im Paradigma des Erklärens und Beweisens - mehr noch: im Paradigma der Kontrolle über die Natur. Dass die Griechen schon nicht den von Crombie unterstellten Begriff von Kausalität verwendeten, sondern eine ganz andere Ursachen- und Prin-

London 1994, S. xxiii.

75 Vgl. zur Entstehung des monumentalen Werks: Ian Hacking: 'Style< for Historians and Philosophers. In: Studies in History and Philosophy of Science, 1 (1992), S. 1-20, hier S. 1.

76 Eine Auseinandersetzung auch mit dem Stilbegriff bei Crombie findet sich in lan Hacking: The Accumulation of Styles and Scientific Reasoning. Companion Piece to sLanguage, Truth and Reason<. In: Steven Lukes/Martin Hollis: Rationality and Relativism, Oxford 1982, S. 48-66; abgedruckt auch in: Dieter Henrich (Hrsg.): Kant oder Hegel? Über Formen der Begründung in der Philosophie, Stuttgart 1983, S. 453-465; außerdem in: Ian Hacking: `Styleく for Historians and Philosophers. In: Studies in History and Philosophy of Science, 1 (1992), S. 1-20.

77 Alistair Cameron Crombie: Commitments and Styles of European. In: Scientific Thinking, History of Science2 (1995), S. 225-238, hier S. 225.

Crombie, 1994, S. 83. 
zipienlehren entwickelten und dass die praktische Kontrolle von Natur gerade nicht mit der griechischen Konzeption von episteme und apodeixes vereinbar ist, vielmehr eine genuin christlich-neuzeitlichen Auffassung darstellt, wird in einer solchen Formulierung von vornherein abgeblendet. So ebnet Crombie vom Standpunkt eines offensichtlich modernen szientistischen Wissenschaftsbildes aus die gravierenden Unterschiede zwischen verschiedenen Wissenschaftstypen ein. Er kehrt gleichsam die Geschichte um: an Stelle einer Beschreibung dessen, wie sich ein dominantes Wissenschaftsbild im Kanon unterschiedlicher Auffassungen von Erkenntnis und Wissen erst herausbildete, setzt er dieses Bild voraus und bügelt die Geschichte des Denkens auf dieses Bild hin glatt.

Durch diese Rückprojektion kann er nicht erkennen, wie sich unterschiedliche Traditionsstränge im späten Mittelalter und in der frühen Neuzeit erst zu einem spezifischen Begriff von scientia bündelten; er kann dadurch auch nicht sehen, dass diese Verbindung von Traditionen nie in einem einzigen Fahrwasser mündete und dass sich neben und gegen das Beweisparadigma die hermeneutischen und naturgeschichtlichen Wissensformen als eigenständige Wissenschaftstypen entfalteten. In seiner Vereinheitlichung und Linearisierung der Wissenschaftsgeschichte bringt er auch diese Typen auf Spur: er missinterpretiert sie unter Vorgaben, die nicht ihre eigenen sind.

Erstes Beispiel: Wenn Crombie über Taxonomien spricht, dann bezieht er sich zunächst auf Platon und Aristoteles als Autoren einer »logic of classification . $^{79}$ Diese Klassifizieren verknüpft er mit dem Begriffspaar Analyse und Synthese so:

»The argument proceeded by the analysis of a phenomenon into its elements, its location in a subject matter with cognate phenomena, then its synthesis from those elements, thus providing an explanation of its occurence. $\aleph^{80}$

Unversehens wird antik-griechisches Denken so in das moderne atomistisch-mechanistische Erklärungsschema übersetzt. Dass es weder das Begriffspaar Analyse/Synthese in diesem Denken gab (siehe BASISWISSEN 8),

79 Crombie, 1995, S. 236.

80 Crombie, 1995, S. 236. 
dass Analyse dort keinesfalls Zerlegung in Elemente bedeutet, sondern das Aufschlüsseln von konstitutiven Prinzipien, dass es deshalb auch keine Idee der Erklärung durch Synthese aus Elementen gab - all dies fällt einer anachronistischen Rückprojektion zum Opfer.

Zweites Beispiel: Statistik. Auch hier findet Crombie wiederum die Quelle der Statistik im griechischen Denken von Wahrscheinlichkeit. Von dort zieht er eine gerade Linie zur »expliziten Entdeckung von statistischen Regelmäßigkeiten im 17. Jahrhundert als einer neuen Form der Regelmäßigkeit, die sich in ausreichend zahlreichen Populationen oder Ereignissen findet « ${ }^{81}$. Sicherlich: auch die Griechen reflektierten schon über Wahrscheinlichkeiten. Allein: diese Reflexion hatte nichts mit Regelmäßigkeiten in großen Zahlen von Populationen und Ereignissen zu tun. Die Kreuzung der modernen Statistik als Staatslehre mit der - inzwischen mathematisierten - Wahrscheinlichkeitstheorie, ist, wie wir sahen (Kapitel 16), ein genuines Produkt des 19. Jahrhunderts.

Drittes Beispiel: Geschichtswissenschaft zu treiben heißt bei Crombie »von allgemeinen Charakteristika diverser existierender Dinge auf eine gemeinsame Quelle in früherer Zeit zu schließen, gefolgt von der Postulierung von Ursachen, die der Diversifizierung dieser Quelle zu Grund liegen. $\aleph^{82} \mathrm{Hier}$ zeigt sich nichts anderes als das positivistische Bild von Geschichtswissenschaften, wie es uns bei Buckle und Mill begegnet ist - und wie es den entschiedenen Widerspruch Droysens hervorrief (siehe Kapitel 17). Doch: eine hermeneutisch ausgerichtete Wissenschaft erscheint unter einer Voreinstellung, wie sie Crombie leitet, nicht einmal am Horizont.

Unter dem Eindruck von Thomas S. Kuhns Theorie wissenschaftlicher Paradigmen hebt Crombie zwar in einem Text von 1995 hervor, Wissenschaft sei nicht monolithisch und Übergänge in Wissenschaftsstilen kämen wissenschaftlichen Revolutionen gleich. Doch an der letztgültigen Einheitlichkeit des wissenschaftlichen Unternehmens, an der Konstanz von Natur und Denkprinzipien und an der einzigartigen Erfolgsgeschichte der europäischen Wissenschaften als Befreiung von der rohen Abhängigkeit von der Natur lässt er keinen Zweifel. Crombies voluminöse Abhandlung ist ein Beispiel dafür, wie ein einseitig-szientistisches Bild von Wissenschaft nicht nur die Ambivalenzen von Wissenschaft und ihren Auswirkungen aus-, sondern

81 Crombie, 1995, S. 236, übers. WK.

82 Crombie, 1995, S. 237, Übers. WK. 
auch die Pluralität von Wissenschaft selbst abblendet. Und vor dem Hintergrund der Konflikte um Deutungshoheit über Wissenschaft, in denen Crombie implizit Stellung bezieht, verwundert es auch nicht, dass sich in diese Lobpreisung der Wissenschaft stets und unverkennbar auch ein autoritärer Ton mischt:

»But nature stays put [bleibt an Ort und Stelle], and thought consistent in its principles. Hence the objectivity of science, like it or not. Science has liberated us from raw dependence on nature. We may celebrate the uniqueness of European scientific thinking among the great intellectual feats of mankind ${ }^{83}$

Wir sehen also: Es gibt Ansätze zur Typisierung von Wissenschaftskulturen, doch es bleibt entweder bei dichotomen Unterscheidungen zweier entgegengesetzter Paradigmen oder bei Entfaltungen unter der Ägide eines Typus. Will man die ganze Breite dessen, was wissenschaftliche Forschung typischerweise ausmacht, in den Blick nehmen, dann muss man offenbar tiefer ansetzen - und mehr Differenz in Kauf nehmen als es Szientisten lieb ist.

83 Crombie, 1995, S. 238. 



\section{Wissenschaftstypen als Tiefenstruktur interdisziplinärer Zusammenarbeit}

\section{Typologie der Wissenschaften: Neun Wissenschaftstypen als Typen mit Familienähnlichkeiten}

Die Gegenstände, die unter einen Begriff fallen, sind nicht durch Eigenschaften verbunden, die allen gemeinsam wären, sondern durch ein Netz von >Familienähnlichkeiten<. Das war die Einsicht, mit der Ludwig Wittgenstein das Begriffsdenken im 20. Jahrhundert revolutionierte. Damit etwas z.B. Spiel heißen kann, muss es nicht bestimmte allgemeine Eigenschaften aufweisen (z.B., dass man um den Sieg spielt oder mit anderen), sondern nur hinreichend viele Gemeinsamkeiten mit einem Fall, der als Spiel gilt. So können wie bei einem Tau, durch das sich kein einziger durchgängiger Faden durchzieht, aber jeder jeweils andere streckenweise übergreift, Phänomene unter einen Begriff fallen, die nur über Zwischenglieder mit anderen verbunden sind. ${ }^{1}$

Mit dieser Auffassung vom Begriff lässt sich auch das Problem der Einheit und Vielheit in den Wissenschaften auflösen. Wissenschaft lässt sich so verstehen als eine Familie von Fällen mit unterschiedlichen, einander teils mehr, teils weniger überlappenden Ähnlichkeiten. Die Wissenschaftstypen zeigen sich durch unterschiedliche Verwandtschaften miteinander verknüpft. Unter dem einen oder anderen Gesichtspunkt kann der eine oder andere Typus als prototypisch erscheinen - jedoch: diese Gesichtspunkte lassen sich wechseln, sodass das Gesamtbild sich jeweils umstellt. Im Wech-

1 Ludwig Wittgenstein: Das Blaue Buch. In: Werkausgabe Bd. 5, hrsg. v. Gertrude Anscombe/E. M. Gertrude/Rush Rhees/Georg H. Wright, Frankfurt/M 1984, S. 37; Ludwig Wittgenstein: Philosophische Untersuchungen, In: Werkausgabe Bd. 1, Frankfurt a.M. 1982, §66. 
sel der Perspektiven zeigen sich unterschiedliche Wissenschaftstypen als gleichwertige Motivations-, Praxis- und Zugangsformen.

Die historische Darstellung offenbarte, dass jeder Forschungstyp in seiner Geschichte motiviert ist und eigene Validierungsmethoden besitzt. Ob z.B. eine Rekonstruktion als wissenschaftliches Ergebnis zählen kann, hängt davon ab, ob die verfügbaren Relikte, Fragmente oder Daten sich >stimmig< in ein Ganzes fügen lassen, das die Form und Beschaffenheit der zusammengesetzten Elemente miterklärt; ob eine Beobachtung oder Erhebung wissenschaftlich valide ist, hängt davon ab, ob die Zählung, Messung, Datierung oder Beschreibung Gegebenheiten von überlagernden Erscheinungen abzulösen, zu sidentifizieren zutragen vermag; ob experimentelle Forschung wissenschaftliche Ergebnisse zeitigt, ist soweit der Fall, als es gelingt, ein Experimentalsystem zu stabilisieren, dass in der kontrollierten Variation ssignifikante Unterschiede erzeugt in Bezug auf theoretisch beschreibbare Strukturen (seien diese Beschreibungen sprachlich oder in einem formalen Symbolismus verfasst, z.B. in der chemischen Formelschreibweise).

Jeder Typus von Forschung besitzt im Frage-Antwort-Zusammenhang von Wissenschaft eine eigene Struktur und einen eigenen Wert. Dabei fügen sich die Wissenschaftstypen nicht etablierten Disziplingrenzen. Nehmen wir die Wissenschaftsform der Kritik: die Reflexion auf Erkenntnis und ihre Bedingungen ist längst nicht mehr nur ein philosophisches Unternehmen, das eigene Validierungskriterien enthält (z.B. ob sich die Darstellung schlüssig auf sich selbst anwenden lässt), sondern auch ein wissens- und wissenschaftssoziologisches, ein historiographisches, ein anthropologisches und ethnographisches; es findet sich überall da, wo kritische Diskurse verwurzelt sind, die subjekt-, ideologie-, historisch-, regional-, gender-, disziplin- oder klassenbedingte Wirkungen in der Erkenntnis reflektieren. Ebenso ist z.B. die Wissenschaftspraxis Rekonstruktion konstitutiver Bedingungen eines Phänomens ebenso forschungsleitend in der Prähistorischen Archäologie (wenn etwa das Phänomen eines um 400000 v. Chr. (sic!) auf exzellente Flugfähigkeit hin designten Speeres die Frage aufwirft, welche sozialhistorischen und anthropologischen Bedingungen dafür vorausgesetzt werden müssen) ${ }^{2}$

2 Vgl. H. Thieme (Hrsg.): Die Schöninger Speere-Mensch und Jagd vor 400.000 Jahren, Stuttgart 2007. 
wie in der Vulkanologie (wenn danach gefragt wird, welches Zusammenspiel geologischer Faktoren einen ungewöhnlichen Ausbruch hervorbrachten).

Wir finden also eine Reihe von Forschungstypen vor, die jeweils einen Eigenwert besitzen und - wie die historische Rekonstruktion zeigte - tief verankerte und historisch gewachsene Ideale von Wissenschaftlichkeit verkörpern. Konflikte um Wissenschaft treten immer da auf, wo dieser jeweilige Eigenwert nicht geachtet wird und wo ein Forschungstypus zur eigentlichen, wahren Form von Wissenschaft erklärt wird. Ob es die logische Beweisform, der technische Erfolg, die Objektivität der brute facts, die Überschreitung des Alltagsverständnisses, die Reflexionstiefe der Argumentation oder die Bewältigung von Datenmengen ist: Jede wissenschaftliche Leistung kann auf den Thron der Wissenschaftlichkeit gehoben und ihr gegenüber alle anderen entwertet werden. Eine solche Vorrangstelle ist aber nicht nur unbegründet, sie verhindert auch interdisziplinäre, genauer: interwissenschaftstypische Kooperation auf Augenhöhe. Das heißt: Sie verhindert, dass jeder Forschungsansatz in einer Kooperation seine eigenen Stärken vollständig einbringen kann.

Wissenschaft als eine Familie von Typen zu begreifen, bedeutet nicht, dass sich diese Typen einem Vergleich untereinander entziehen. Wie auch für Familienmitglieder Vergleichsgrößen bestehen (Haarfarbe, Nasenform, Körperbau, Augenfarbe etc.), so gibt es auch für die Wissenschaftstypen Größen, in Bezug auf die sie sich vergleichen lassen - wodurch sich die Familienähnlichkeiten allererst ja zeigen. Mithilfe solcher Vergleichsgrößen lassen sich Gemeinsamkeiten und Unterschiede aufzeigen, ohne diese Unterschiede als Unterschiede des Wertes oder der Entwicklung zur Wissenschaftlichkeit aufzufassen. Sie sind als rein typologische Unterschiede zu begreifen.

Das bedeutet methodologisch, dass nur neutrale und wertfreie Vergleichsinstanzen zum Einsatz kommen können. Ein solches Tertium Comparationis muss ausreichend struktural und praxisbezogen angelegt sein, es darf nicht ein spezifisches Wissenschaftsideal abbilden.

Als Vergleichsinstanzen in diesem Sinne bieten sich an:

1. die Art der Fragen, die einen Wissenschaftstypus kennzeichnen

2. die Praktiken, die ihn charakterisieren

3. die Form dessen, was in einem Typus als wissenschaftliches Ergebnis gilt 
In diesen drei Hinsichten lassen sich neun Grundkonzeptionen von Wissenschaft voneinander unterscheiden:

1. Gegenstandserfassender Forschungsansatz: Daten zu erheben, ist eine Grundform von Wissenschaft. Wie werden Daten erhoben? Indem fragliche Elemente beobachtet, gezählt, gemessen, datiert, verortet oder beschrieben werden. Die einfachste Form besteht in Fragen der Art: Wie häufig tritt $\mathrm{x}$ auf? Wo tritt $\mathrm{x}$ auf? Wann tritt $\mathrm{x}$ auf? Mit Instrumenten lassen sich Fragen nach Temperatur, Masse, elektrische Leitfähigkeit, Alter eines Elementes verfolgen. Aufzeichnungssysteme wie Zeitstrahlen, Karten, Tabellen und Diagramme dienen der Erfassung der Daten.

2. Typisierend-ordnungsgenerierender Forschungsansatz: Während in der gegenstandserfassenden Variante das Element $\mathrm{x}$ selbst nicht infrage steht, wenn z.B. ein Planet verortet oder eine Chronologie von Herrschern erstellt wird, stellt sich im typisierend-ordnungsgenerierenden Ansatz die Frage nach der Bestimmung von x: Welchem Typ, welcher Art, welcher Gattung, welcher Epoche, welchem Genre ist x zuzuordnen? Die Basis-Praxis dieses Forschungsansatzes ist das Vergleichen: Welche Merkmale finden sich im einen Exemplar, welche im anderen? Welche Typen sind $\mathrm{zu}$ unterscheiden? Welche Typen sind allgemeiner, welche spezieller? Wie ist das Ordnungssystem als Ganzes aufgebaut? Ergebnisse finden hier üblicherweise ihre Darstellungen in Klassifikationsschemata, komparatistischen Erörterungen und synoptischen Taxonomien.

3. Auch die klassische Statistik bewegt sich in ihrer Grundform im Rahmen dieses Forschungsparadigmas. In der statistischen Variante werden Vorkommnisse nicht nur typisiert, sondern zugleich in ihrer Häufigkeit erfasst. Damit eröffnet sie ein ganz eigenes Forschungsfeld: das der Häufigkeitsverteilung. Es lassen sich nun Häufigkeiten von Ereignissen in Bezug auf andere Ordnungsfragen vergleichen: Wie sieht es in welcher Zeit aus? Wie in welcher Gegend? Wie bezogen auf welche Bevölkerungsanteile etc.? Statistik produziert damit ein Wissen zweiter Ordnung: ein $\mathrm{x}$ wird als Gegenstand zuordnend erfasst (erste Ordnung) und sodann in seiner Häufigkeit in Hinsicht auf ein zweites Einteilungssystem in vergleichende Darstellung gebracht (zweite Ordnung). Statistisches Wissen ist zum allergrößten Teil tabellarisches und diagrammatisches Wissen.

4. Rekonstruierender Forschungsansatz: Die Grundfrage hier ist: Wie sah ein Ganzes aus, wenn nur Relikte, Indizien, Spuren, Fragmente vorhanden 
sind? Die Forschungsform ähnelt hier der Untersuchung eines Kriminalfalls: ein Ablauf, ein Geschehen, ein Zusammenhang soll als eine schlüssige Ganzheit rekonstruiert werden. Das kann dann in der Form eines Rekonstruktionsmodells wie etwa in der Archäologie (materiell oder digital), in Form einer Narration wie in manchen geschichtswissenschaftlichen Studien oder in Form einer Strukturbeschreibung geschehen. Die bestimmende Praxis ist hier die versuchsweise Kombination im Sinne von: Wie passt $\mathrm{x}$ mit y zusammen? Was ergäbe sich für das Ganze, wenn $\mathrm{x}$ als a und $y$ als $b$ betrachtet würde? Und wenn das Ganze so oder so aussähe, dann wäre wiederum das Element $\mathrm{z}$ als $\mathrm{c}$ zu betrachten; kann das sein? Diese Frageform des Rätsellösens in einem Teil-Ganzes-Setting verbindet - in ihrer Form, nicht im Gegenstand - historische Rekonstruktion mit Textauslegung und manchen Formen der Experimentalforschung: In jedem Fall versucht man, am Einzelnen eine sTheorie « über einen Gesamtzusammenhang zu entwickeln und an einem (vorausgesetzten oder hypothetisch entworfenen) Gesamtzusammenhang Verhalten und Beschaffenheit von Elementen als integrale Bestandteile zu deuten.

5. Rekonstruktiv-erklärender Forschungsansatz: Sind nicht Indizien, Spuren und Relikte gegeben, die auf ihren vormaligen Zusammenhang hin gedeutet werden müssen, sondern ein als solches gegebenes oder vorausgesetztes Phänomen, z.B. die Entstehung einer Revolution, das überraschende Verhalten eines chemischen Stoffes, der Klimawandel, eine Krankheit o.ä., dann richtet sich die Forschung auf das Bedingungsgefüge, unter dem das Zustandekommen von x zu erklären ist. Was muss als gegeben angenommen werden, dass das (erstaunliche, fragwürdige) Phänomen x zustande kommt? Die Forschung richtet sich hier auf die in einem Bedingungsgefüge zusammenwirkenden Faktoren, die am Entstehen einer Ganzheit, eines Phänomens in je zu bestimmender Weise beteiligt sind. Weil ein solches Bedingungsgefüge, z.B. für den Klimawandel, für ein historisches Ereignis oder für das Entstehen von menschlicher Sprache extrem komplex sein kann, hat auch hier die Forschung einen stark heuristischen und experimentellen Charakter. Zum Einsatz kommen unterschiedliche Mittel der Modellierung, die das Bedingungsgefüge probeweise zusammenstellen und Abläufe, die infrage stehen, probeweise simulieren. Solche Modellierungen werden typischerweise auf komplexe Naturvorgänge angewandt, aber auch immer häufiger in Sozialwissenschaften eingesetzt. 
6. Der Forschungsansatz der interpretativen Adäquation: Während im Labor ausdrücklich mit künstlichen Bedingungen gearbeitet wird, gewinnt die Forschung im Paradigma der Adäquatheit ihre Validität dadurch, dass sie den Phänomenen in ihrem Aspektreichtum durch Anpassung der Darstellungsmittel möglichst umfassend gerecht $\mathrm{zu}$ werden sucht. In einer Linie mit dem Gedanken der adaequatio intellectu et rei kondensiert sich die Bemühung um die Anpassung der seigenen vorausgesetzten Konzepte und Darstellungsmittel an den Gegenstand in einem eigenen Forschungsansatz. Die leitende Frage lautet: Wie müssen Begriffe, Konzepte, Regelformulierungen verändert werden, damit sie einen Gegenstand in seiner Phänomenalität zum Vorschein bringt, ohne konstitutive Elemente und Aspekte zu vernachlässigen? Wenn z.B. in der Ethnologie nach der Bedeutung einer sozialen Praxis gefragt wird oder in der Philologie nach der angemessenen Übersetzung eines altsprachlichen Wortes, dessen Bedeutungsaspekte aus einer Fülle von Kontexten gewonnen worden sind, besteht die Aufgabe darin, eine diesen Aspekten angemessene Interpretation zu geben. Eine angemessene Interpretation wiederum muss nicht in einer passenden Übersetzung münden, tatsächlich ist ein Aufdecken der Übersetzungsproblematik und die Notwendigkeit einer beschreibenden Auslegung das typische Resultat einer auf Adäquation gerichteten Forschung.

7. Der Forschungsansatz der Kritik. Dass jedes Wissen oder Gedachte reflexiv $\mathrm{zu}$ erfassen und zu durchdringen ist und dass es unter je spezifischen Bedingungen zustande kommt, dass zudem die Aufklärung und Reflexion der Bedingungen zumindest ein zentrales Moment von gewissenhafter Forschung darstellt, dies ist das Grundmotiv der kritischen Forschungspraxis. Ihre Praxisform lässt sich als die des Herausschälens, Aufspürens, Entlarvens und Aufdeckens beschreiben. Verborgene, unbemerkte, vernachlässigte oder verdrängte Einflussfaktoren werden detektiert und nach vorn gestellt. Erkenntniskritik, Ideologiekritik und Dekonstruktion sind Namen für Ansätze in diesem Paradigma Kritischer Forschung. Wenn die jeweils aufgedeckten Zusammenhänge als `wahre Motive ‘ hinter dem Schein der Oberfläche aufgefasst werden, hat dieser Ansatz Ähnlichkeiten mit dem Reduktionismus vom analytisch-apodiktischen Typus: In beiden Fällen wird dann der Phänomenbestand als Schein auf das Sein einer wahren Struktur zurückgeführt. Richtet er sich aber auf das Bedingungsgefüge von Erkenntnis, ohne darin universale Gesetzmäßigkeiten 
anzunehmen, dann zeigt er sich als eine genuine Form phänomenbezogener Forschung - eine solche nämlich, die die Erscheinungsweise des Phänomens in seinen Erscheinungsbedingungen offenlegt.

8. Reduktiv-apodiktischer Forschungsansatz: Geht man davon aus, dass sich das Bedingungsgefüge, aus dem sich ein Gesamtzusammenhang erklärt, restlos von jeweiligen Erscheinungen lösen und als universales und gesetzmäßiges Regelwerk in einer formalen Sprache darstellen lässt, dann ergibt sich eine andere Form der Grundfrage: Wie lässt sich ein phänomenaler Zusammenhang auf einen universalen Formalismus zurückführen? Die wissenschaftliche Praxis besteht hier in erster Linie darin, eine formale, logische oder mathematische Sprache so aufzubauen, dass sich in ihr die infrage stehenden Eigenschaften abbilden lassen; man spricht heute gerne von mapping. Eine solche Praxis ist zurzeit am häufigsten im Bereich der Linguistik, der Neurowissenschaften und der Genetik anzutreffen. Gemeinhin ist sie in erster Linie Programm, wie im Bereich der Kognitionswissenschaften. Da diese Wissenschaftsform im Einklang mit den metaphysischen Voraussetzungen der atomistisch-mechanistischen Deutung der Beweislehren steht, die die Wissenschaftsphilosophie lange Zeit geprägt und noch immer starken Einfluss auf Hintergrundüberzeugungen hat, bestimmt sie stärker ein bestimmtes Bild von Wissenschaften als tatsächliche Forschungspraxis.

9. Der Ansatz der experimentellen Laborwissenschaften: In diesem Forschungsansatz werden symbolsystemische Formalismen nicht als Zielpunkt der Forschung eingesetzt, auf den phänomenale Zusammenhänge zurückgeführt werden (Reduktion), sondern als Forschungsinstrumente. Der Formalismus wird im Labor nicht schlicht bestätigt, sondern eingesetzt, um Experimentalsysteme zu strukturieren, die sich darauf richten, noch unbekannte Eigenschaften, Wirkfaktoren und Interdependenzen im Gegenstandsbereich zu entdecken und zu beschreiben. Die zentrale Praxis ist die der Einrichtung von möglichst stabilen Experimentalsystemen, sodass einzelne Faktoren kontrolliert und variiert werden können. Zur Ergebnissicherung gehört hier niemals nur die Angabe eines RegelFormalismus (’Erklärung), sondern stets die Beschreibung des Experimentalsystems, des technischen Versuchsaufbaus und der idealisierten Bedingungen, unter denen sich die Zusammenhänge beobachten ließen. Es ist dieser Forschungstyp, der die Frage der Reproduzierbarkeit aufwirft. 


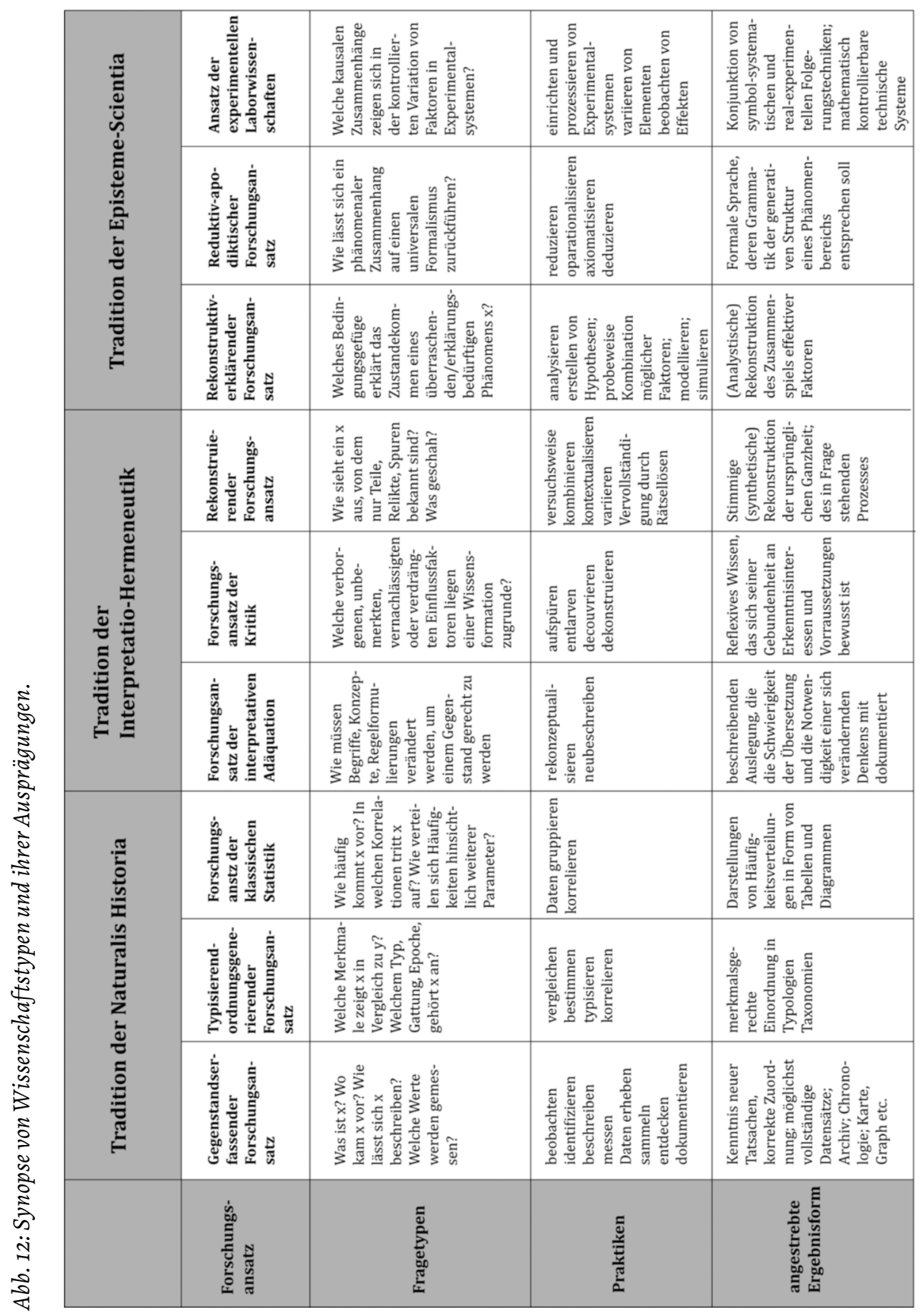


Synoptisch lassen sich somit neun Typen von Wissenschaft in einem Schema nebeneinanderstellen (Abb. 12).

Diese Typologie ist aus historischen Rekonstruktionen gewonnen und stellt eine Möglichkeit dar, Wissenschaftstypen als jeweils eigenständige Praxis- und Motivationskomplexe zu beschreiben. Die Darstellungsform hat den Vorteil, auf einen Blick das Nebeneinander von Typen sichtbar zu machen und sie regt zu Vergleichen an. Sie hat aber auch Nachteile, die in der Bildlogik liegen: Zum einen mag es erscheinen, dass, bedingt durch unsere Leserichtung von rechts nach links, wir es mit einer Folgeordnung zu tun haben, die doch eine Richtung zu höherer Integration und Komplexität und damit eine implizite Hierarchie suggeriert. Zum anderen erschwert die serielle Anordnung Vergleiche von entfernt voneinander angeordneten Wissenschaftstypen. Um diese Nachteile zu umgehen, wäre eine dreidimensionale Darstellung vorzuziehen - etwa in der Art von Abb. 13.

Eine solche freiere Darstellung stellt uns nun vor die Frage nach den möglichen Verhältnissen der Wissenschaftstypen zueinander. Wenn diese Darstellung geeignet ist, ein hierarchisches Bild der Verhältnisse zwischen wissenschaftlichen Motivations-Praxis-Typen abzuwenden und damit die notorischen Kämpfe um Deutungshoheit zu überwinden, dann eröffnet sie die Möglichkeit von Kooperationen auf Augenhöhe: sie verbindet sich nun mit dem Themen- und Aufgabenkomplex der Interdisziplinarität.

Abb. 13: Wissenschaftstypen in nicht-serieller Darstellung.

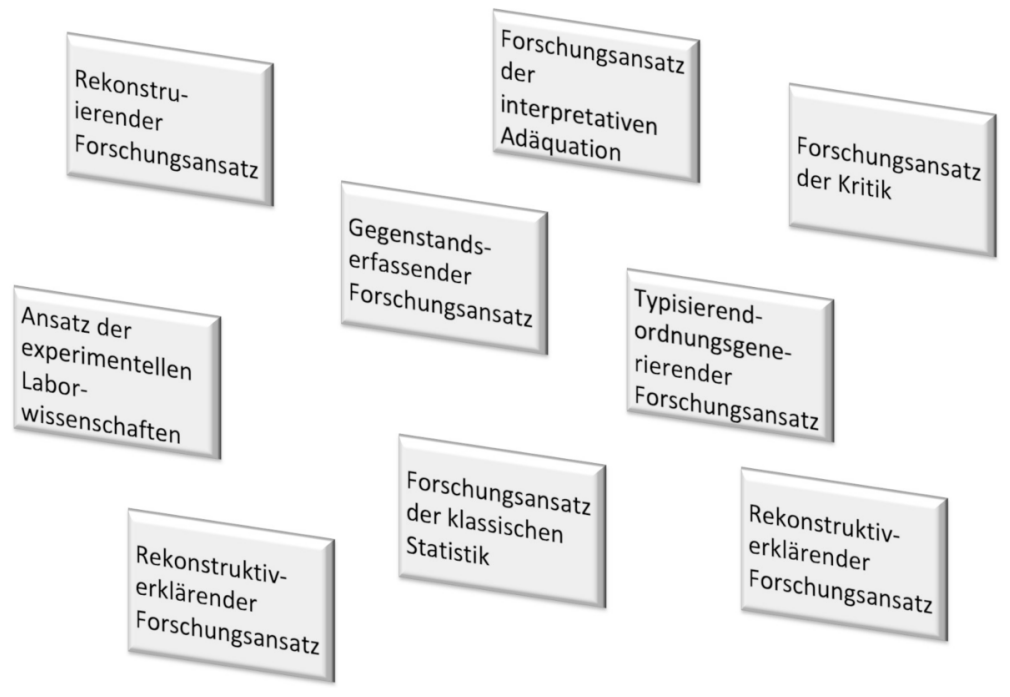




\section{Die Pluralität der Wissenschaften und das Problem der Interdisziplinarität}

Begonnen haben wir mit der Frage, wie es um Einheit und Vielfalt der Wissenschaften steht und angeschlossen haben wir die Frage, wie unter der Bedingung einer Pluralität von Wissenschaftstypen Zusammenwirken gedacht und ermöglicht werden kann. Der klassische Leitbegriff, unter dem dieses Thema verhandelt wird, ist Interdisziplinarität. Die Forderung nach interdisziplinärem Arbeiten durchzieht die gesamte Literatur der Forschungsanträge und wissenschaftsplanerischen Projekt- und Strategiepapiere. Doch in der Praxis erweist sich Interdisziplinarität als Herausforderung. Die damit verbundenen Schwierigkeiten und Konflikte sind seit längerem benannt und aufgelistet. So spricht etwa eine Studie zum Management von Forschungsverbünden ${ }^{3}$ von den Schwierigkeiten interdisziplinärer Zusammenarbeit in deutlichen Worten:

»Einige der Schwierigkeiten erwachsen aus der Tatsache, dass Inter- und Transdisziplinarität eine Kooperation zwischen disziplinären `Kulturen ‘ darstellt. Die Angehörigen dieser disziplinären `Kulturen verfügen über eine je eigene Sprache, die sich von derjenigen anderer Disziplinen und von der Alltagssprache unterscheidet, und sie bedienen sich disziplinenspezifischer Theorien und Methoden. Ebenso haben sie je eigene Vorstellungen über das rrichtige< Vorgehen. Fachsprache, Theorien und Methoden sind Ausdruck einer disziplinenspezifischen Weltsicht und Handlungsweise. ${ }^{4}$

Daraus entstehen in der Darstellung dieser Studie eine Reihe von Konflikten:

»Der Wissenskorpus und die damit zusammenhängende Sprache der eigenen Disziplin gehören oft zum lediglich impliziten Wissen einer Person. Sie enthalten vieles, was innerhalb der eigenen Disziplin als Selbstverständlichkeit gilt. [...] Entsprechend schwierig ist es, eine gemeinsame Sprache zu finden. Konflikte entstehen, wenn die Beteiligten darauf bestehen, ihre

3 Rico Defila/Antonietta Di Giulio/Michael Scheuermann (Hrsg.): Management von Forschungsverbünden-Möglichkeiten derProfessionalisierung und Unterstützung, DFC Standpunkte, Weinheim 2008. Zu weiterer Literatur zur Problematik interdisziplinärer Kooperation vgl. S. 17. 4 Defila u.a., 2008, S. 18. 
Verwendungsweise eines Wortes sei die >richtige`, und nicht bereit sind, von ihrem Begriffsverständnis abzurücken, sondern auf einem Definitionsprimat beharren.«

Solche Konflikte betreffen nicht nur das Problem einer gemeinsamen Sprache, sondern bereits Divergenzen in der Auffassung von »Gegenstand und Fragestellung«:

»Das Wissen darüber, welche Aspekte eines (vorwissenschaftlichen) Gegenstands für eine Untersuchung relevant sind und wie sie beschrieben werden - also die Konstruktion des Forschungsgegenstands - ist Teil der disziplinenspezifischen 〉Brille`, die sich Wissenschaftlerinnen und Wissenschaftler aneignen. [...] Konflikte entstehen, wenn die Beteiligten überzeugt sind, ihre Sicht sei die einzig mögliche Sicht auf den jeweiligen (vorwissenschaftlichen) Gegenstand, sie allein würden die srichtigen und wichtigen Fragen aufwerfen und wenn sie die Fragen der anderen als weder sinnvoll noch zielführend abqualifizieren.«

Ebenso Differenzen auf der Ebene der »Methodenwahl und Wissenschaftlichkeit«:

»In der inter- oder transdisziplinären Forschung muss auf disziplinäre Methoden der Erkenntnisgewinnung zurückgegriffen werden. Die disziplinären Vorstellungen über das >richtige` Vorgehen, über Erfolg versprechende Methoden und die angemessene Art und Weise, an eine Frage heranzugehen, divergieren aber. Dasselbe gilt für die Auffassungen darüber, welche Kriterien der Wissenschaftlichkeit anzuwenden sind. [...] Konflikte entstehen, wenn sich die Beteiligten weigern, von ihren Kriterien der Wissenschaftlichkeit Abstand zu nehmen, und mit aller Macht versuchen, ihre Methoden und ihre Wissenschaftlichkeitskriterien durchzusetzen.«

Häufig spielen auch ganz grundsätzliche »Vorurteile« eine Rolle:

»Konflikte entstehen, wenn jemand besser zu wissen meint, was andere Disziplinen leisten können oder sollten, als die Angehörigen dieser Disziplinen, und wenn dies mit >Disziplinenimperialismus einhergeht, d.h. mit der Überschätzung des Beitrags der eigenen Disziplin bei gleichzeitiger Her- 
absetzung der Leistungen anderer Disziplinen (bis hin zur Negierung ihrer Wissenschaftlichkeit). $\ll^{5}$

Entsprechend hört man in interdisziplinären Forschungsverbünden hinter vorgehaltener Hand viel Unmut und Skepsis angesichts der interdisziplinären Herausforderung und es gibt offensichtlich eine verbreitete Sehnsucht, sich ins vertraute Terrain des ureigenen Materials, der beherrschten Methoden, der gewohnten Fragestellungen zurückziehen zu dürfen. Doch die Fliehkraft von sich immer weiter ins Spezielle ausdifferenzierenden Wissenschaftsdisziplinen hält im Gegenzug den ebenso starken Impuls am Leben, den Zusammenhang des Wissens wieder gewinnen zu wollen. Wie kann die eine Welt verstanden sein, wenn sie in disziplinäre Einzelwelten zerfällt, für die nicht einmal Weichenstellungen, Anschlüsse oder Übersetzungswerkzeuge in Sicht sind?

Während das frühe 19. Jahrhundert eine Vision einer Einheit des Wissens als Integral universaler Gelehrsamkeit kannte und die Phase von Mitte des 19. bis Mitte des 20. Jahrhundert als die Hochzeit der wissenschaftlichen Einheitskämpfe unter je spezifischen Deutungsansprüchen erscheint, stehen seitdem zwei Auffassungen einander gegenüber: diejenige, die Wissenschaftskulturen als je in sich motivierte, autonome Denkstile oder Wissenschaftsparadigmen sehen und diejenige, die ein Integral der Interdisziplinarität forcieren. Während die einen den Eigenwert eines jeden wissenschaftlichen Denkens großmütig in den Blick nehmen und dabei ihre Inkommensurabilität, ${ }^{6}$ also wechselseitige >Unanmessbarkeit<, konstatieren, steuern die anderen auf ein Integral zu, das stets Gefahr läuft, der pluralen wissenschaftlichen Realität ein äußerliches Programm überzustülpen.

5 Die vorangegangenen Zitate finden sich bei Defila u.a., 2008, S. 18-20.

6 Vgl. Kurt Bayertz: Das Problem der Inkommensurabilität. In: Wissenschaftstheorie und Paradigmabegriff, Stuttgart 1981, S. 77-105; Paul Hoyningen-Huene: Inkommensurabilität bei Kuhn und Theorienvergleich. In: E. Agazzi (Hrsg.): Die Vergleichbarkeit wissenschaftlicher Theorien, Freiburg 1990, S. 97-108. 


\section{Wie ist diesem Dilemma zu entkommen?}

Die Analyse von Wissenschaftstypen könnte die Antwort auf diese Frage sein. Das Stichwort heißt hier Tiefenstruktur. Wissenschaftstypen verhalten sich zu Disziplinen wie die Bewegung von Kontinentalplatten zum Oberflächenrelief. Hier wirken - um im Bild zu bleiben - die Formierungskräfte wissenschaftlicher Praxis, die die Disziplinen durchzieht. Sie bilden die Ebene, auf der sich bestimmt, was in der Forschung tatsächlich geschieht. Und zwar: was einen Forschungstyp in sich motiviert und wie er seine Frage-, Herangehens- und Darstellungsweisen ausrichtet auf das, was in ihm als Erkenntnis und Wissen gilt. Elementare Handlungsmuster wie die des Messens, Erörterns, Vergleichens, Berechnens, Mischens etc. spielen hier entscheidende Rollen. Doch auf der Ebene der Wissenschaftstypen sind sie in Motivkomplexe intergiert: man misst, um Daten zu gewinnen, die in einem bestimmten Fragekomplex und Darstellungssystem eine bestimmte instruktive Aussage z.B. in Bezug auf Klimawandel bedeuten. Oder: Man analysiert grammatische Formen, um zu untersuchen, wie ein Text sprachstrukturell von Leitgedanken seiner Epoche geprägt ist, was ein Bewusstsein für historische Spezifität schafft. Wissenschaftstypen zu beschreiben bedeutet, Praktiken in Motivationskomplexen zu erfassen. Solche Motivationskomplexe sind, wie gesehen, historisch gewachsen und prägen sich in verschiedenen Formen aus. Diese Komplexe durchziehen die Disziplinen.

Manchmal schlägt sich dies in Bezeichnungen nieder: physische Geographie versus Sozialgeographie; hypothesengeleitete Laborforschung versus Systembiologie; quantitative Soziologie versus Kultursoziologie (um einige Beispiele zu nennen). Meist aber machen sich solche Differenzen in der Tiefenstruktur nur als Spannung und Konflikt bemerkbar. Die Tiefenstruktur offenzulegen birgt die Chance in sich, ausdrücklich und aktiv mit solchen Spannungen umzugehen.

Doch wie kann auf der Ebene der Wissenschaftstypen das Problem der Interdisziplinarität gelöst werden? Wie können hier Grenzen überwunden und Forschung zusammengeführt werden? Ziel aller Interdisziplinarität ist ein Surplus, ein Synergieeffekt, der aus der Verbindung der Perspektiven entsteht. Interdisziplinarität ist nicht Selbstzweck, sondern zielt auf umfassendere Erkenntnis, auf ein breites aufgebautes Wissen. Ein Standardargument lautet: Komplexe Probleme in einer komplexen Welt erfordern komplexe Forschung. Nur: Wie kann eine solche komplexere Forschung aufgebaut 
werden? Die Forschenden wachsen in ihren Disziplinen auf, lernen in ihnen nicht nur eine Gegenstandssphäre und Methoden, sondern auch eine Sprache und - das ist vielleicht entscheidend - die Raison d'etre ihrer Forschung kennen; also: was macht sie sinnvoll, was macht sie spannend? Konfrontiert mit anderen Disziplinen, in denen eine Sprache gesprochen wird, die sich nie ganz in die eigene Forschungssprache übersetzen lässt und in Angesicht von Motiven, die quer oder schräg liegen zu denen, die die eigene Forschung ausrichten, wachsen, trotz anfänglichem Enthusiasmus, Hilflosigkeit und Frust.

Betrachtet man nun aber Wissenschaftstypen als Tiefenstrukturen von Disziplinen, dann zeigt sich eine neue Chance: die Ausrichtung der Forschung selbst, ihre Verschachtelung in Teilabschnitte und Prozessschritte wird hier zur Gemeinsamkeit; indem Forschungsmotive miteinander verknüpft und - in ihrer Gleichwertigkeit - auf verschiedene Weisen aufeinander aufgebaut werden, entstehen komplexe Forschungsformen, die in sich integriert sind. Wo tatsächlich die tiefen Differenzen liegen, da finden sich auch die Gelenkstellen, die verknüpfte Forschung mit Mehrwert erlauben.

\section{VERTIEFEND Konzepte der Interdisziplinaritätsforschung}

Um diesen Gedanken zu verdeutlichen, wird es hilfreich sein, einen Blick ins Feld von Interdisziplinaritätsforschung und Interdisziplinaritätsstudien zu werfen. Denn diese Metadisziplinen sind genau deshalb entstanden, weil sich Interdisziplinarität zwar als erstrebenswert, aber keineswegs als unproblematisch erwies. Die Fragen und die Antworten in diesem Feld betreffen daher direkt unsere Problemstellung. Die Differenzierungen, die hier vorgenommen werden, sind Versuche, das Problem in den Griff zu bekommen. Doch stimmt die These, dass das Problem der Forschungsintegration erst auf der Ebene der analysierten Wissenschaftstypen lösbar ist, dann müsste sich das auch in Bezug auf dieses Feld zeigen.

\section{Womit haben wir es zu tun?}

Interdisziplinaritätsforschung und Interdisziplinaritätsstudien haben sich seit den 1990er Jahren zu einem eigenen Forschungsfeld entwickelt und eine eigene Terminologie ausgeprägt. So unterscheidet man Multidisziplinarität, Interdisziplinarität und Transdisziplinarität. Multidisziplinär ist ein Forschungsverbund dann, wenn mehrere Disziplinen in ihm vereinigt sind, 
die jeweils ihre Perspektive auf den Forschungsgegenstand beisteuern; Interdisziplinarität setzt darüber hinaus voraus, dass die unterschiedlichen Perspektiven in eine gemeinsame Forschungsanstrengung integriert sind, was bedeutet: jede Disziplin nimmt auch andere Perspektiven auf und verändert sich in ihrer Forschungsweise; transdisziplinär heißt Forschung dann, wenn interdisziplinäre Forschungsgruppen zusätzlich außerwissenschaftliche Akteure integrieren, z.B. aus NGOs, Nutzer- und Betroffenenverbänden, Politik, Wirtschaft, Gewerkschaften etc.

Bei interdisziplinärer Verknüpfung unterscheidet man des Weiteren nach Verbünden von Disziplinen, die sich traditionell nahestehen (Narrow ID) und solchen, zwischen denen große Distanzen vermutet werden (Broad/Wide ID). ${ }^{7}$

Die interessanteste Differenzierung liegt nun aber auf der Ebene des Mediums, in dem sich Disziplinen integrieren. Ist es ein gemeinsames Problem, eine gemeinsame Theorie, ein gemeinsames Objekt oder eine gemeinsame Methode, in der sich unterschiedliche disziplinäre Forschungsperspektiven zusammenfinden? ${ }^{8}$ Der stärkste politische Impuls, der in Richtung Interdisziplinarität drängt, liegt aktuell sicherlich in der Problemorientierung: Klimawandel, Urbanisierung, Diskriminierung, Wasserknappheit, Hunger, Naturkatastrophen, Pandemien, Luftverschmutzung, Ressourcenknappheit - viele solcher Themen zeigen Problemstellungen, die an keine einzelne Disziplin delegiert werden kann. Das zentrale Argument liegt hier im Verweis auf Komplexität: Solche Probleme sind so vielgestaltig, dass sie der multiperspektivischen und zugleich integrierten Anstrengung vieler Disziplinen bedürfen.

Doch diese Differenzierung von Problem-, Objekt-, Theorie- und Methodenorientierung interdisziplinärer Forschung, die Jan C. Schmidt eindrück-

7 Julie Thompson Klein: A taxonomy of interdisciplinarity. In: Robert Frodeman/Julie Thompson Klein/Carl Mitcham/J. Brott Holbrook (Hrsg.): The Oxford Handbook of Interdisciplinarity, Oxford 2010, S. 15-30, hier S. 18.

8 Vgl. Margaret A. Boden: What is interdisciplinarity? In: R. Cunningham (Hrsg.): Interdisciplinarity and the organization of knowledge in Europe, Office for Official Publications of the European Communities, Luxembourg 1999, S. 13-24; H. Bruun,/J. I. Hukkinen/K. I. Huutoniemi/]. Thompson Klein: Promoting Interdisciplinary Research: The Case of the Academy of Finland. In: Publications of the Academy of Finland, 8 (2005); Jan C. Schmidt: Towards a philosophy of interdisciplinarity. An attempt to provide a classification and clarification. In: Poiesis and Praxis 1 (2008), S. 53-69; Jan C. Schmidt: What is a problem? On problem-oriented interdisciplinarity, Poiesis and Praxis 4 (2011), S. 249-274. 
lich ausbuchstabiert, gibt allein noch kein Werkzeug zur Integration an die Hand. Das zeigt sich, wenn wir die Orientierungen durchgehen:

Zunächst: Ein gemeinsames Problem mag in vielen Fällen Anlass sein, aber sie macht noch keine gemeinsame Forschung. Problemorientierung scheint ein viel zu breites Anliegen zu sein, um Formen wissenschaftlicher Integration daraus zu entwickeln. Übergreifende Probleme, die sich politisch artikulieren, sind zunächst nicht die Probleme, an denen sich disziplinäre Forschung ausrichtet. Problemorientierung zeigt die Notwendigkeit der Integration und gegenseitigen Übersetzung an; sie gibt aber noch kein Werkzeug an die Hand.

Kommen wir also, zweitens, zur Objektorientierung. Objektorientierung bedeutet, eine bestimmte Objektklasse zu privilegieren. Man spricht hier von einer ontologischen, also die Ordnung des Seins betreffenden Herangehensweise. Der seit der Antike verhandelte Atomismus ist eine solche Position und wir haben im vorhergehenden Abschnitt gesehen, dass es auch im 20. Jahrhundert Ansätze gab, eine Einheit der Wissenschaften durch Rückgang auf physikalische Elemente zu begründen. Der Ansatz der Unity of Science, demgemäß Geistiges, Soziales und Biologisches auf physikalische Einheiten zurückzubeziehen sei (siehe Kapitel 18) stand in dieser atomistischphysikalistischen Tradition. Für jüngere Tendenzen gibt Jan C. Schmidt ein treffendes Beispiel, wenn er einen Report der US-amerikanischen Wissenschaftspolitik zitiert, in dem Nanoobjekte als Basis einer interdisziplinären Forschungsverbundes aus Nano-, Bio-, Informations- und Kognitionswissenschaften betrachtet werden. ${ }^{9}$ Objektorientierte Interdisziplinarität zeigt sich auch hier als reduktive Strategie: Die Wahrheit der Phänomene liegt in den Eigenschaften der kleinsten Teile.

Doch wie sieht es mit Theorie- und Methodenorientierung aus? Die Idee hier ist, dass eine gemeinsame übergreifende Theorie oder eine basale Methodologie das gesuchte Integral liefern könnte. Kandidaten sind hier aufseiten der Theorie System- und Selbstorganisationstheorien, Theorien komplexer Systeme und Chaostheorien, aufseiten der Methoden mathematische Modellierungen, Bionics und ähnliches. ${ }^{10}$

9 Vgl. Schmidt, 2011, S. 266. Vgl. auch: Werner Kogge: Technologie des 21. Jahrhunderts. Perspektiven der Technikphilosophie. In: Deutsche Zeitschrift für Philosophie, 6 (2008),

S. 935-956.

10 Vgl. Schmidt, 2011, S. 254-256. 
Blickt man auf diese Kandidatenliste, so zeigt sich Theorie- und Methodenorientierung als Integral eines Forschungsprogramms, das tatsächlich den umfassendsten und aufwändigsten Ansatz darstellt, der je in Sachen interdisziplinärer Forschung veranstaltet wurde. Wenn ein Nature-Artikel von $2015^{11}$ als Institutionen mit Pioniercharakter solche aufzählt, die Informatik, Mathematik oder neurowissenschaftliche Ansätze auf andere Disziplinen anwenden (Santa Fe Institute (von 1984); Janelia Research Campus), dann ist hier zu ergänzen: Dieser Typ von Interdisziplinarität hat eine lange, starke Tradition, die die gesamte Epoche des Kalten Krieges beherrschte:

Es war der Komplex aus Kybernetik, Informationstheorie und mathematische Theorien biologischer Automaten, der seit Ende der 1940er Jahre durch eine Reihe von Publikationen Popularität erlangte: 1948 erschienen sowohl Norbert Wieners Cybernetics or control and communication in the animal and the machine und Claude Shannons The Mathematical Theory of Communication, 1949 und 1952 trat John von Neumann mit seinen Vortragsreihen The Theory and Organization of Complicated Automata und Probabilistic Logics and the Synthesis of Reliable Organisms from Unreliable Components an die Öffentlichkeit; ${ }^{12}$ des Weiteren waren die zehn interdisziplinären Macy-Konferenzen, die zwischen 1946 und 1953 stattfanden, wichtiger Katalysator für die Verschmelzung von biologischen und mathematischen Konzeptionen. Unter dem Leitbegriff $\mathrm{Cy}$ bernetics wurden Mathematik, Biologie, Computerwissenschaften, Neurologie, Maschinenlehre, aber auch Linguistik, Psychologie und Anthropologie in ein gemeinsames Feld geführt. Das generelle Thema dieser Ansätze sind Regelsysteme und die Steuerung von Verhalten; das Organische gilt dabei als Vorbild und als potenzieller Gegenstand der Entwicklung mathematischer Modelle und technischer Systeme. ${ }^{13}$ Was den Einsatz von Mitteln und die Einbeziehung von Forschungsfeldern anbelangt, ist das kybernetische Integral sicherlich das größte interdisziplinäre Forschungsprogramm, das es je gab; seine Verflechtung mit militärischen Zielvorgaben, zunächst noch im Zweitem Weltkrieg, dann im Kalten Krieg, ist viel diskutiert. Gründungen

11 Heidi Ledford: Team Science. In: Nature, 525 (2015), S. 308-311.

12 Vgl. Lily E. Kay: Das Buch des Lebens: Wer schrieb den genetischen Code?, München 2002, S. 159.

13 Vgl. Kay, 2002; Evelyn F. Keller: Das Leben neu Denken. Metaphern der Biologie im 20. Jahrhundert, München 1998; Des Weiteren: Peter Galison: Die Ontologie des Feindes. Norbert Wiener und die Vision der Kybernetik. In: Michael Hagner (Hrsg.): Ansichten der Wissenschaftsgeschichte, Frankfurt a.M. 2001, S. 433-485; Michael Hagner/Erich Hörl (Hrsg.): Die Transformation des Humanen, Frankfurt a.M. 2008. 
interdisziplinärer Institutionen wie die im Nature-Artikel zitierten stehen fast sämtlich in diesem ideellen und pragmatischen Kontext.

Mit welcher Form von Interdisziplinarität haben wir es bei Verbünden in diesem Programm zu tun? Sie ist nicht objektorientiert, denn sie ist ontologisch offen; sie ist auch nicht problemorientiert, denn die Ausrichtung auf Steuerung und Kontrolle richtet sich eher auf eine Problemlösungsstrategie als auf bestimmte Probleme. Spezifisch wird sie aber auf theoretischer und methodischer Ebene. Die Kybernetik beruht nämlich auf voraussetzungsreichen theoretischen Annahmen, die in ihrem Paradigma der Selbstorganisation impliziert sind. Das im Grunde Aristotelische Konzept (Stichwort entelecheia: Zielformation von natürlichen Wesenheiten) wurde in der Biologie des 18. Jahrhunderts (Blumenbach: Stichwort Bildungstrieb) neu aufgegriffen, in Kants Philosophie des Organischen ausbuchstabiert (Kritik der Urteilskraft; Zweiter Teil) und auf unterschiedlichen Wegen ins 20. Jahrhundert tradiert, wo es mit der mathematischen Theorie rekursiver Funktionen und neuen computertechnischen Möglichkeiten verschmolzen wurde. So entstand aus einem klassischen Theorem, das am Phänomen des Organischen gewonnen war, ein umfassendes Forschungsprogramm, das sich von der Kybernetik $\mathrm{zu}$ unterschiedlichen Ausprägungen von Systemtheorie weiterentwickelte. Paul Varelas und Hubert Maturanas Terminus der Autopoiesis (also der Selbstbildung), der grundlegend wurde für Niklas Luhmanns Theorie sozialer Systeme, spiegelt noch den Ursprung dieser theoretische Traditionslinie.

Doch lässt sich durch gemeinsame Theorie Interdisziplinarität herstellen? Kann es eine solche die Disziplinen überspannende gemeinsame Theorie überhaupt geben? Das kybernetische Paradigma ist ein Beispiel dafür, dass dies für bestimmte übergreifende Interessen wie das Bedürfnis nach Steuerung und Kontrolle möglich ist. Doch es ist auch ein Beispiel dafür, dass ein solches Paradigma von vornherein die Potentiale vieler Wissenschaften ausschließt. Denn den Gegenstand als ein rekursiv gesteuertes oder selbstorganisierendes System zu betrachten, läuft in vielen Forschungskontexten ins Leere. Das Gleiche gilt auf der Ebene der Methode für den Einsatz mathematischer Modellierung in Theorien der Selbstorganisation bis hin zu Komplexitäts- und Chaostheorien.

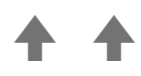


Der vertiefende Blick in die Interdisziplinaritätsforschung zeigt eine Problematik, die sich so zusammenfassen lässt:

Für die Gemeinsamkeit, die verschiedene Disziplinen in ein Forschungsvorhaben integriert, kommen vier Kandidaten infrage: eine gemeinsame Problemstellung, ein gemeinsames Objekt (Forschungsgegenstand), gemeinsame Methoden oder gemeinsame Theorien. Diese vier Kandidaten werfen jeweils eigene Probleme auf: Die gemeinsame Problemorientierung zeigt lediglich den Bedarf an interdisziplinärer Verbindung an, gibt aber noch keine Mittel an die Hand, wie eine solche zu bewerkstelligen sein kann; das Gleiche gilt für gemeinsame Gegenstände, wenn es schließlich darum geht, unterschiedliche disziplinäre Perspektiven auf ein Objekt in zusammenhängende Erkenntnis zu überführen; außerdem stellt sich die Frage, ob das vermeintlich gemeinsame Objekt in den disziplinären Perspektiven nicht völlig unterschiedlich konzeptualisiert ist - auch hier bleibt die Frage nach der Basis der Gemeinsamkeit unbeantwortet. Gemeinsame Methoden und gemeinsame Theorien auf der anderen Seite werfen die Frage auf, ob es sich überhaupt um unterschiedliche Disziplinen handeln kann, wenn sie in einem einzigen methodisch-theoretischen Horizont arbeiten. Denkbar ist so etwas in einer Art Meta-Methodik und Meta-Theorie. Und genau dafür gibt es im 20. Jahrhundert ein Beispiel, das das größte und vielleicht auch einzige umfassend realisierte Forschungsprogramm methodischer und theoretischer Interdisziplinarität darstellt: Von den 1950er bis in die 1990er Jahre bildeten die Theorien von Steuerung, Selbstorganisation und Ordnungsbildung ein theoretisches Dach, das methodisch mit mathematischer und informationstechnischer Modellierung arbeitete. Kybernetik, Systemtheorie, Komplexitätstheorie, Chaostheorie, mathematische Modellierung und Fuzzy Logic sind Titel für Teile und Stufen dieses Forschungsprogramms. ${ }^{14}$

Jedoch: Die Integration in eine Methodik, die Phänomenbereiche mathematisch-formalistisch zu fassen sucht, ist, wie wir sehen konnten, nur ein bestimmter Typus von Wissenschaft. Für viele Forschungsgegenstände ist er ungeeignet und vielen Disziplinen fremd. Wir haben es also weniger mit einer Anleitung zu interdisziplinärer Kooperation denn mit einer Übersetzung in

14 Damit ist nicht gesagt, dass sich nicht jüngere Ansätze von Systemtheorien diesem Modell auch entziehen; Es wäre aber zu prüfen, ob sie sich damit gegenüber dem ursprünglichen Forschungsprogramm nicht isolieren; ein zu prüfendes Beispiel wäre etwa die soziologische Systemtheorie nach Niklas Luhmann. 
ein spezifisches Forschungsprogramm zu tun. Ebenso ist das theoretische Erkenntnisinteresse der Steuerung komplexer, dynamischer Ordnungen - so allgemein es gehalten ist - doch ein spezifisches. Für viele wissenschaftliche Fragestellungen spielt dieser Theorieansatz überhaupt keine Rolle.

Mit diesen Überlegungen deutet sich an, dass die Frage der Integration von Erkenntnissen als Frage der Interdisziplinarität noch nicht tief genug gestellt ist. Disziplinen sind als Produkte vielfältiger historischer Entwicklungen in sich selbst methodisch, theoretisch und gegenständlich divers und untereinander undeutlich abgegrenzt. Die wirksamen Unterschiede liegen auf der Ebene der Wissenschaftstypen, die die Disziplinen durchziehen.

Auf dieser Ebene der Wissenschaftstypen zeigt sich nun aber, dass die Suche nach einer gemeinsamen Basis für integriertes Arbeiten eine letztlich unmöglich zu realisierende und auch unnötige Voraussetzung macht: nämlich, dass Integration genau da und so weit gelingt, wie Gleichheit vorausgesetzt oder Angleichung durchgesetzt werden kann.

Mit dem Theorem der Familienähnlichkeiten (der Wissenschaftstypen) kommt hier eine neue Denkfigur zum Vorschein: die Figur der ergänzenden und fortführenden Verknüpfung selbständiger unterschiedlicher Einheiten anstelle der Figur der einheitlichen Basis und Angleichung.

\section{Noch einmal zum Konzept der Wissenschaftstypen: Idealtypen mit Anschlussstellen. Ein modulares Konzept interdisziplinärer Zusammenarbeit}

Wissenschaftstypen als historisch gewachsene Motivkomplexe zu analysieren, bedeutet auch, sie jeweils als eigenständig und eigenwertig zu betrachten. In ihnen selbst liegt das Potential, gemäßihrer Motive validiertes Wissen zu produzieren, also solches, das sich an Wertungskriterien der jeweiligen Wissenschaft bemisst. Schaut man aber auf Wissenschaftstypen vor dem Hintergrund der Interdisziplinaritätsthematik, dann stellt sich die Frage, ob Wissenschaftstypen Solitäre sind, die sich der Zusammenarbeit untereinander entziehen. Einen Gedanken zur Auflösung der Spannung zwischen Autonomie und Zusammenhang haben wir im Kapitel 20 angesprochen: das Wittgensteinsche Theorem der Familienähnlichkeiten, mit der Metapher des Taus, in dem sich viele einzelne Fasern überlappen und zu einem starken Tau verbinden, ohne dass eine einzelne Faser als die sallgemeine das gesamte 
Tau durchzieht. Dieses Konzept, das einen neuartigen Begriff vom Begriff hervorbrachte, hat befreiende Wirkung: Es entbindet von der Aufgabe, die allgemeinen definitorischen Eigenschaften von Wissenschaft aufzusuchen, ein Unterfangen, das in sich stets die Tendenz birgt, partielle Sichtweisen mit hegemonialen Ansprüchen hervorzubringen.

Eine anti-hierarchische Sicht auf die Pluralität der Wissenschaften findet im Theorem der Familienähnlichkeit eine gute Grundlage. Was allerdings in diesem Theorem noch nicht ausgedrückt ist, ist die Antwort auf die Frage, wie die Verknüpfung der Einzeltypen vonstattengehen kann. An dieser Stelle steht im Wittgensteinschen Kontext das Konzept der Analogie. Analogie (ursprünglich ein Begriff der Aristotelischen Metapherntheorie) bezeichnet im Kern, dass sich durch die Entdeckung gemeinsamer Eigenschaften sehr unterschiedlich erscheinende Gegenstände miteinander ins Verhältnis stellen lassen und sich damit zeigt, wie sie zueinanderstehen.

Die unterschiedlichen Wissenschaftstypen sind ein Anwendungsfall analogischer Verknüpfung. In ihren Eigenschaften treten unterschiedliche Gemeinsamkeiten und partielle Überlappungen auf. Diese jeweils anderen Gemeinsamkeiten bilden, entdeckt man sie und legt sie frei, die Andockstellen für wissenschaftliche Kooperation.

Um diesen Gedanken zu verdeutlichen, ist es hilfreich, ein weiteres Theorem aufzurufen: Max Webers Konzept der Idealtypen. Idealtypen lassen sich als paradigmatische, also mustergültige Fälle begreifen, die tatsächlich vorkommen, aber selten in Reinform, und die vor allem als ein Vergleichsobjekt dienen, um eine Vielfalt von Phänomenen beschreiben zu können - kontrastiv. Wissenschaftstypen sind solche Idealtypen. Es gibt Fälle, in denen wissenschaftliche Forschungsprojekte vollständig innerhalb eines wissenschaftlichen Typus angelegt sind. Für gewöhnlich ist Forschungspraxis aber ein Tätigkeitskomplex, in dem sich unterschiedliche typische Modalitäten kombiniert finden. Dies geschieht unter einem Leitmotiv, sodass sich verschiedenartige wissenschaftstypische Fragen und Motive häufig im Gesamtrahmen eingeschachtelt finden.

Und es scheint fast so, als ob solche Einschachtelungen von Wissenschaftstypen sich restlos als Stationen in wissenschaftlichen Forschungsprozessen verstehen lassen (vgl. Abb. 14). Spielen wir das einmal durch: (1) Man beobachtet, vermisst, beschreibt einen Gegenstand, sodann (2) vergleicht man ihn, ordnet ihm einen Typus, einem Genre, einer Epoche zu, klassifiziert ihn, bestimmt (3) die Häufigkeit seines Auftretens in Bezug auf be- 
stimmte Korrelationen, somit die empirische Vorkommensverteilung; setzt dann mit einer Interpretation an, in der das Phänomen im Zusammenhang von Teil und Ganzem rekonstruiert wird, wobei der Schwerpunkt (4) auf der Synthese von Anzeichen, Spuren, Relikten oder (5) auf der Analyse möglicher Bedingungen liegen kann, (6) arbeitet man daran, Begriffe, Interpretationen, Modelle konzeptuell anzupassen, (7) werden kritisch die Bedingungen reflektiert, unter denen Gegenstandsbestimmungen, Typisierung, Statistik und Interpretation zustande gekommen sind, was mit Aussagen über Reichweite der Erkenntnisse und über notwendige weitere Forschungen und veränderte Programme verbunden ist; (8) reduziert man den interpretierten Gesamtzusammenhang auf ein universales Regelwerk, das sich idealiter in einer formalen Sprache ausdrücken lässt, oder versucht (9) das verhandelte Phänomen unter Laborbedingen zu reproduzieren (um schließlich zu einer praktisch-technischen Anwendung zu kommen).

Man könnte so zu einem Bild kommen, demgemäß die Wissenschaftstypen nicht wirklich separate und autonome Forschungseinheiten darstellen, sondern unterschiedliche Schritte oder Stationen in einem einheitlichen Forschungszusammenhang. Ein Verlaufsdiagramm von Forschung könnte demgemäß so aussehen:

Betrachten wir aber die Rhetorik, in der wir dieses Ablaufschema entworfen haben und die Bildlogik, in der wir es dargestellt haben, dann zeigt sich die Suggestion, mit der ein solches Schema stets verbunden ist: Das Handeln scheint hier von anfänglichen, elementaren Schritten zu hochkomplexen und subtil-reflektierten Tätigkeiten fortzuschreiten. Es scheint, als ob wir von der Kärrnerarbeit im Heizkeller der Forschung immer höher in die sauberen Tätigkeiten der Weißkittel-Etagen aufsteigen. Wenn diese shöheren< Tätigkeiten dann auch noch in hoch-artifiziellen und technisch voraussetzungsreichen Sprachen wie etwa der shöheren Mathematik oder der poststrukturalistischen Philosophie ausgedrückt werden, dann scheint es, als ob es sich in diesem Prozess um einen Gang von primitiven Vorarbeiten zu elaborierter Erkenntnis handelt.

Doch das ist, wie gesagt: alles Suggestion; geschuldet bestimmten Vorurteilen darüber, was wissenschaftliche Erkenntnis im Kern letztlich ausmacht; Quelle all der Status- und Deutungskämpfe, die sich durch die Geschichten der Wissenschaften ziehen.

Dass die Verschachtelung der Wissenschaftsmotive und Fragestellungen nicht als ein lineares Aufstiegsschema verstanden werden kann, dass es sich 


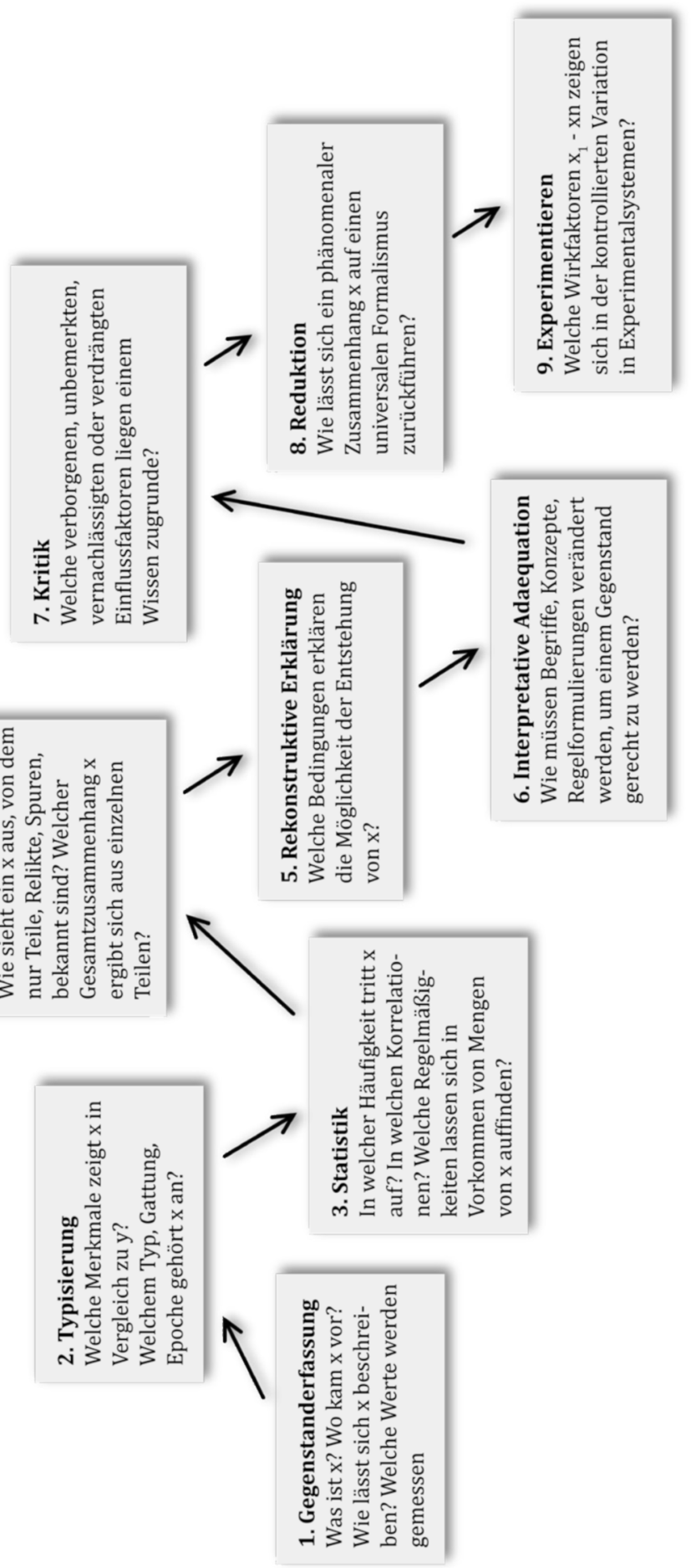


vielmehr um ein modulares System handelt, in dem sich Forschungsprozesse auf verschiedene Weisen kombiniert finden, offenbart sich nämlich in der Tatsache, dass man die Richtungen umkehren kann (vgl. Abb. 15). So gibt es Forschungsprojekte, in denen Bedingungen rekonstruiert werden (5), um zu einer Typisierung (2) zu gelangen; Laborversuche (6) werden angestellt, um Phänomene z.B. chronologisch oder geographisch (1) verorten zu können; unbefragte Voraussetzungen von Forschungsprogrammen werden befragt (7), was zu einer Neubeschreibung des Gegenstandes (1) führt.

Um der Suggestion zu entkommen, ist es wiederum hilfreich, die Darstellungs- und Bildlogik in eine konsequent-flächige zu transformieren, in der auf einer Ebene unterschiedliche Verknüpfungsformen gleichwertig erscheinen.

Schon diese Andeutungen weisen darauf hin, dass jeder Wissenschaftstyp das Erkenntnisziel eines konkreten Forschungsprogramms bestimmen kann. Das Motiv eines jeden Typus kann die Zielstellung der Arbeit ausmachen, sodass wir zu einem Bild der Kombinierbarkeit kommen, eine egalitäre Modularität sozusagen.

\section{Egalitäre Modularität: Was ist damit gemeint?}

Es bedeutet, dass typische wissenschaftliche Praxis-Motiv-Komplexe sich als Module auffassen lassen, das heißt als selbständige Funktionseinheiten, die in einem komplexen Ganzen integriert sind. Das komplexe Ganze ist hier ein konkretes Forschungsprojekt. Solche Forschungsprojekte sind typischerweise modular aufgebaut. Allerdings wird dieser modulare Aufbau selten als solcher wahrgenommen. In den Projektbeschreibungen verschwindet er unter Rubriken wie Vorarbeiten und Forschungsprogramm. Was wäre, wenn dies nicht geschähe? Wie sähe dies aus?

Wären Forschungsprojekte so zu entwerfen, dass ihr modularer Aufbau expliziert werden würde, dann hätte dies eine ganze Reihe von Effekten zur Folge:

1. Der Eigenwert der Forschungsschritte wird sichtbar, das heißt jedes solches Element wird als eigener Typus von Wissenschaft Beachtung finden, was die Sorgfalt der Planung, Ergebnissicherung usw. befördern kann;

2. es wird sichtbar, dass bestimmte modulare Einheiten, wie z.B. eine kritische Reflexion oder eine typisierende Einordnung in einem Projekt fehlt, obwohl es für die Gesamtfragestellung erforderlich sein könnte; 


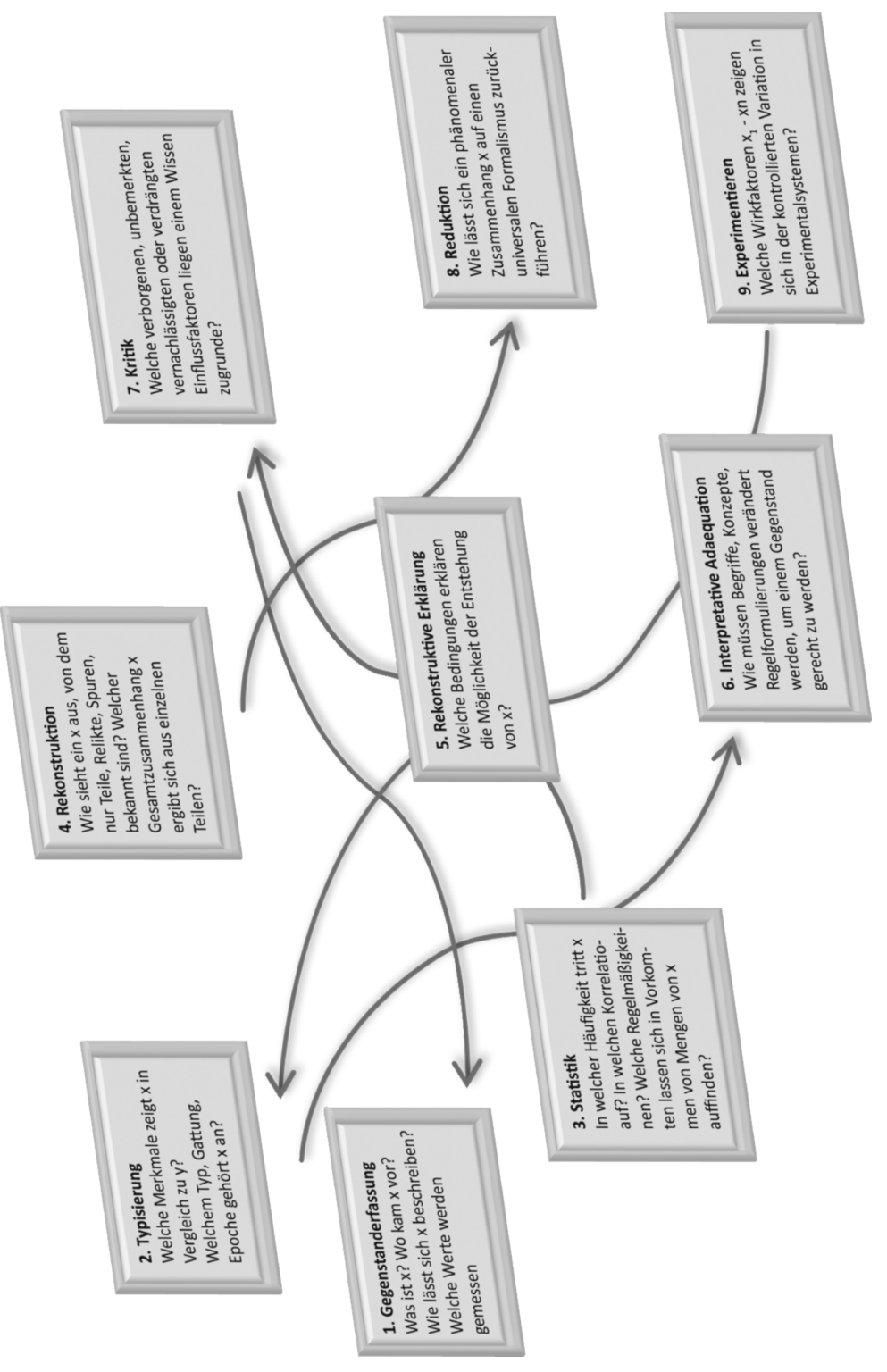




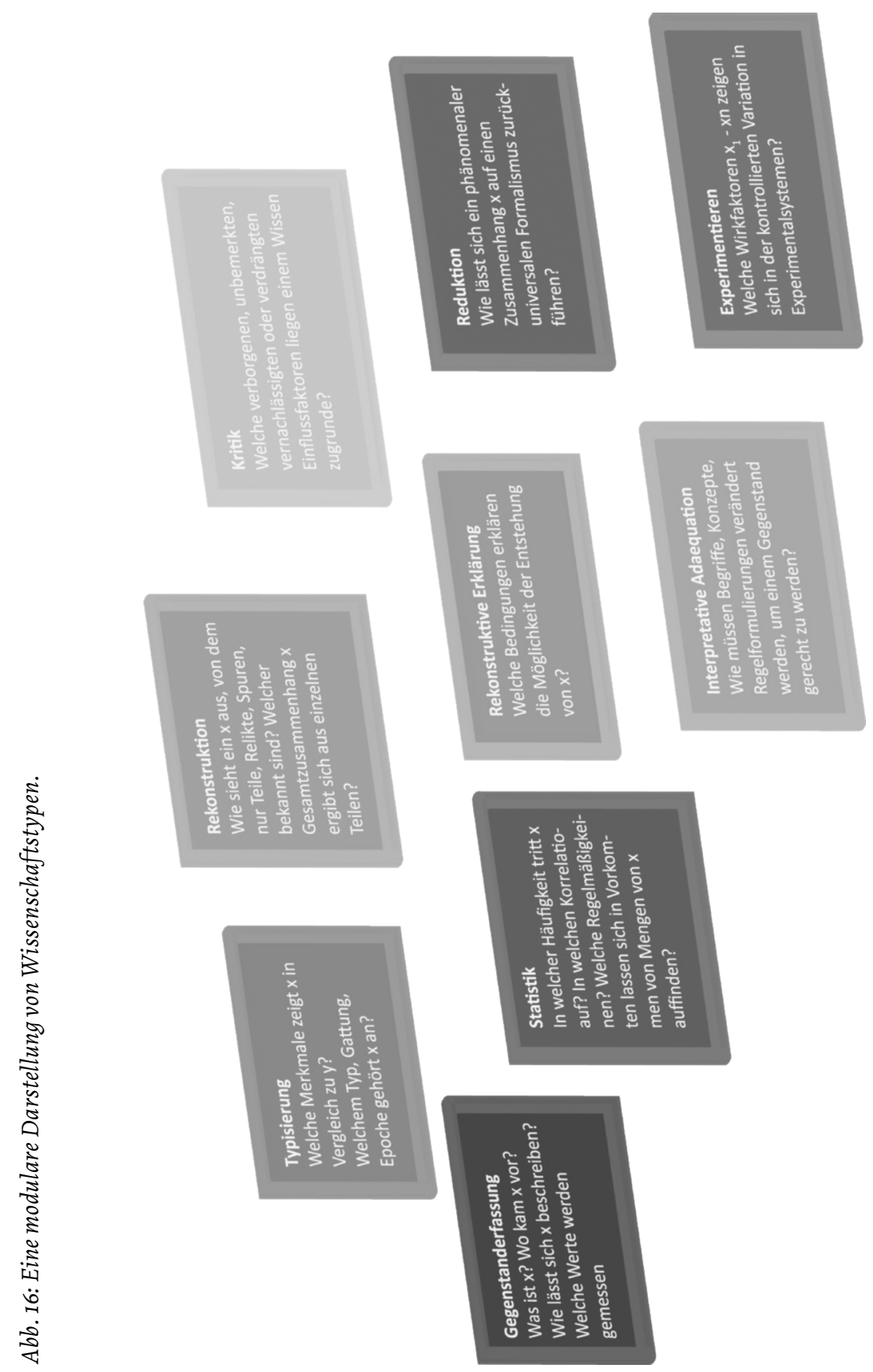


3. Begründungen, welche Module wie miteinander verknüpft werden, werden explizit, damit reflektier- und verhandelbar.

Insgesamt würde eine solche modulare Sichtweise dazu führen, Forschungsprojekte weitaus bewusster $\mathrm{zu}$ entwerfen. Man könnte sagen: die Gestaltungstiefe von Forschungsprojekten reicht dann viel tiefer; und zwar bis zur Ebene der Strukturen, in denen ihre Praxen wurzeln und motiviert sind.

Der Aufbau von Forschungsprojekten aus eigenständigen Modulen impliziert eine ganz neue Tätigkeit im Forschungsdesign: Planung bedeutet hier auch eine elementare Kombinatorik. Es geht um die Auswahl der Modulelemente - welche sind wichtig? - und um ihre Anordnung. Dass Forschungsprozesse motiviert sind, und dass sie Zielstellungen enthalten, die jeweils durch die übergreifende Fragestellung eines Wissenschaftstypus geprägt sind, dieser Aspekt bleibt erhalten. Was sich ändert, ist zweierlei: zum einen wird klar, dass jede übergreifende wissenschaftstypische Leitfragestellung eine optionale Wahl ist. Das heißt, sie ist nicht dadurch bedingt, dass ein wissenschaftliches Ergebnis diese oder jene typische Form hat. Die Starrheit, die die wissenschaftlichen Deutungskämpfe in die Forschungen tragen, wird somit überwunden. Zum anderen steht stets infrage, und erfordert eine Begründung, ob bestimmte Module in ein Forschungsprojekt eingebaut oder nicht eingebaut werden. Nicht jedes Forschungsprojekt muss aufgeblasen werden, um die Gesamtheit der modularen Elemente zu integrieren. Aber in der Forschungsplanung die Liste der Module durchzugehen und darzulegen, welche Rolle sie jeweils im konkreten Projekt spielt oder nicht spielt, das kann zu einer entscheidend vertieften und weit umfassender reflektierten Forschungsplanung führen. Das schließt Fragen ein wie diejenige, welche Forschungsansätze in Ergänzung mit- und zueinander stehen sollten und wie unterschiedliche Forschungsansätze aufeinander abgestimmt werden.

Schauen wir uns exemplarisch drei sehr einfache Beispiele für die modulare Anlage von Forschungsprojekten an. (Abb. 17)

Diese einfachen zweischritten Anlagen von Forschungsprojekten sind der Übersichtlichkeit der Darstellung geschuldet. Reale Forschungsprojekte werden im Durchschnitt eine komplexere Modularität aufweisen, also aus der Kombinatorik von mehreren Wissenschaftstypus-Bausteinen bestehen.

Eine modulare Anlage von Forschungsplanung bedeutet auch ein neu ausgerichtetes Programm von Interdisziplinarität. Denn wenn in der Forschungsplanung bestimmte Module als sinnvoll erachtet und eingebaut 


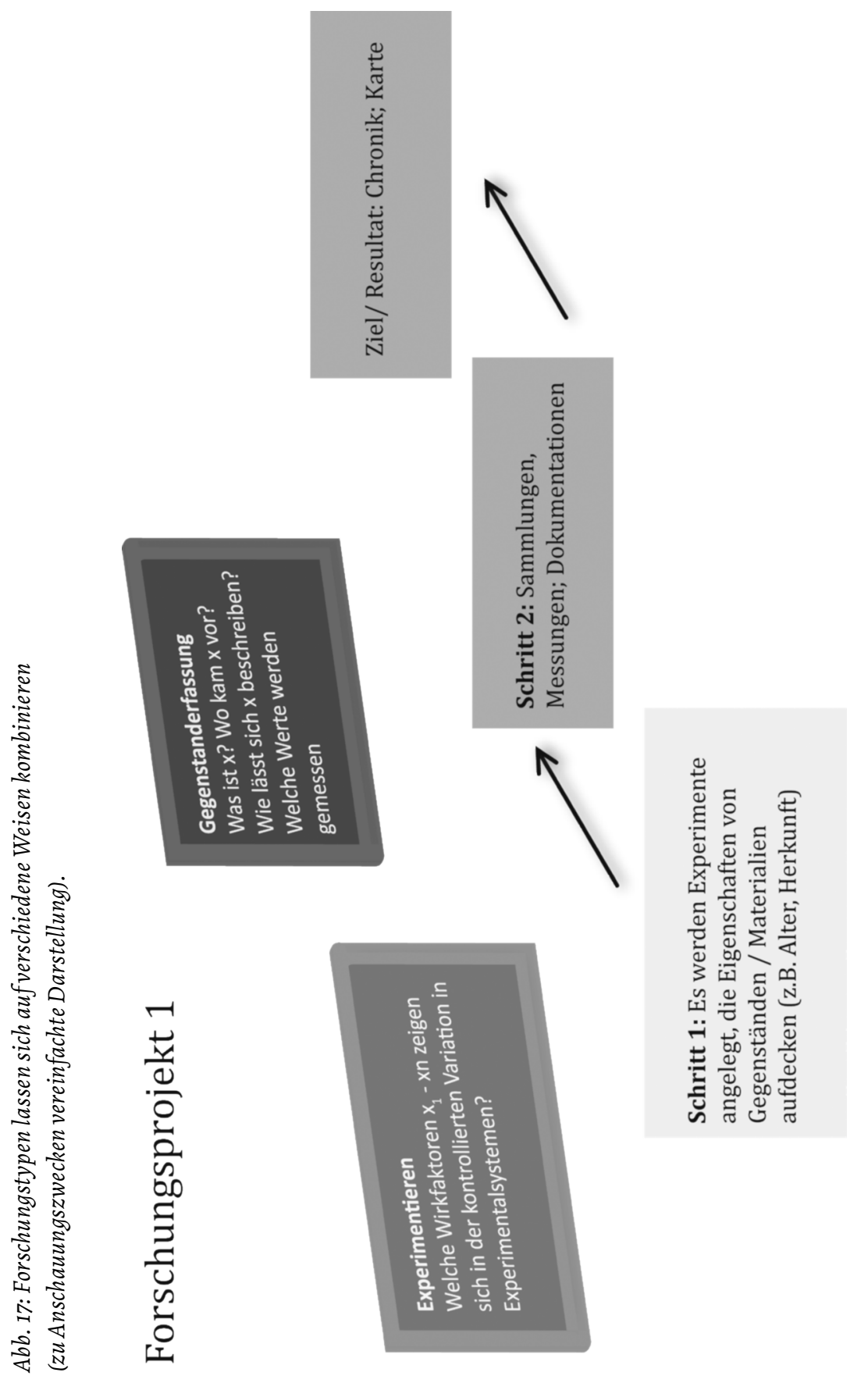




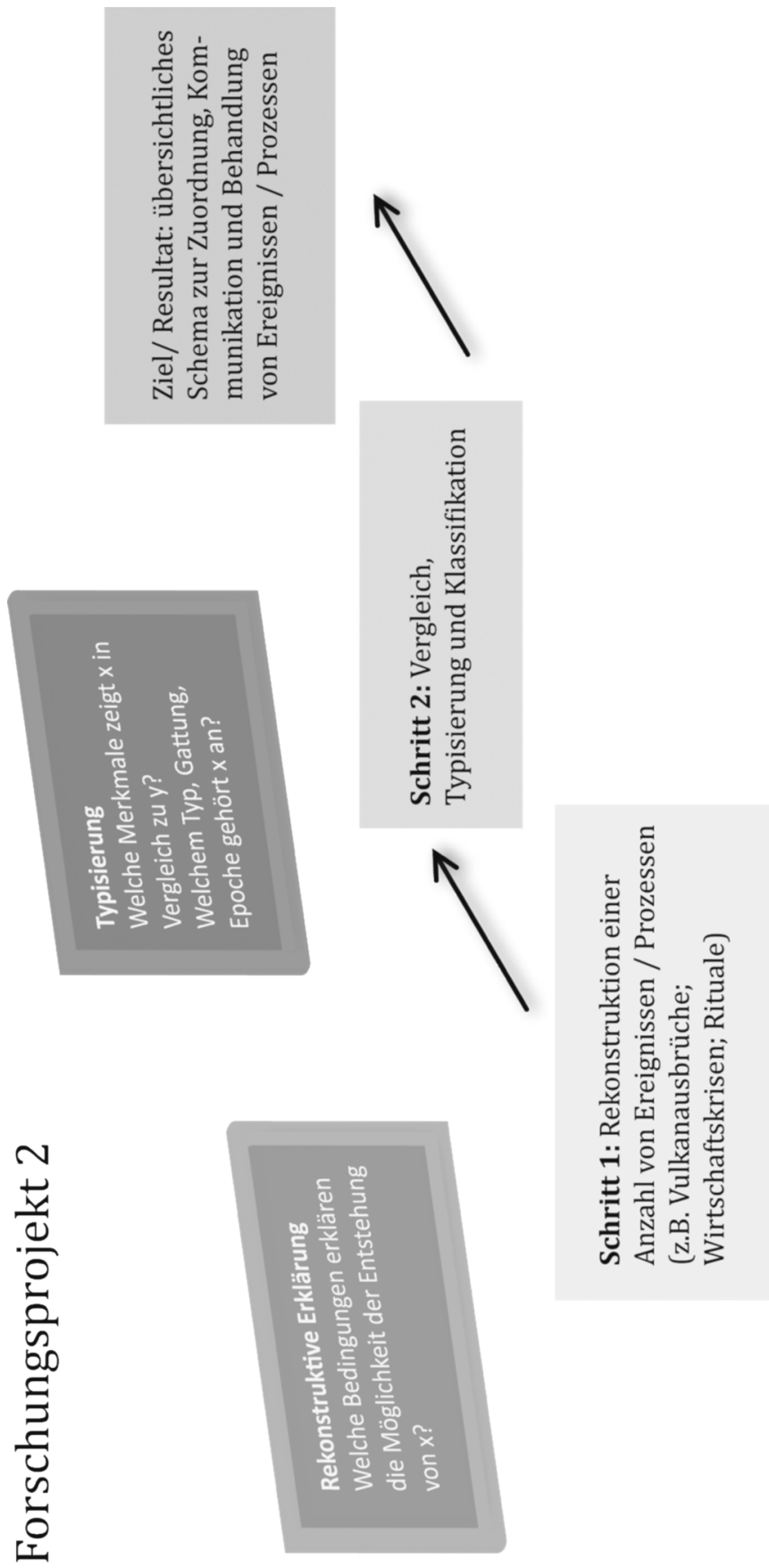




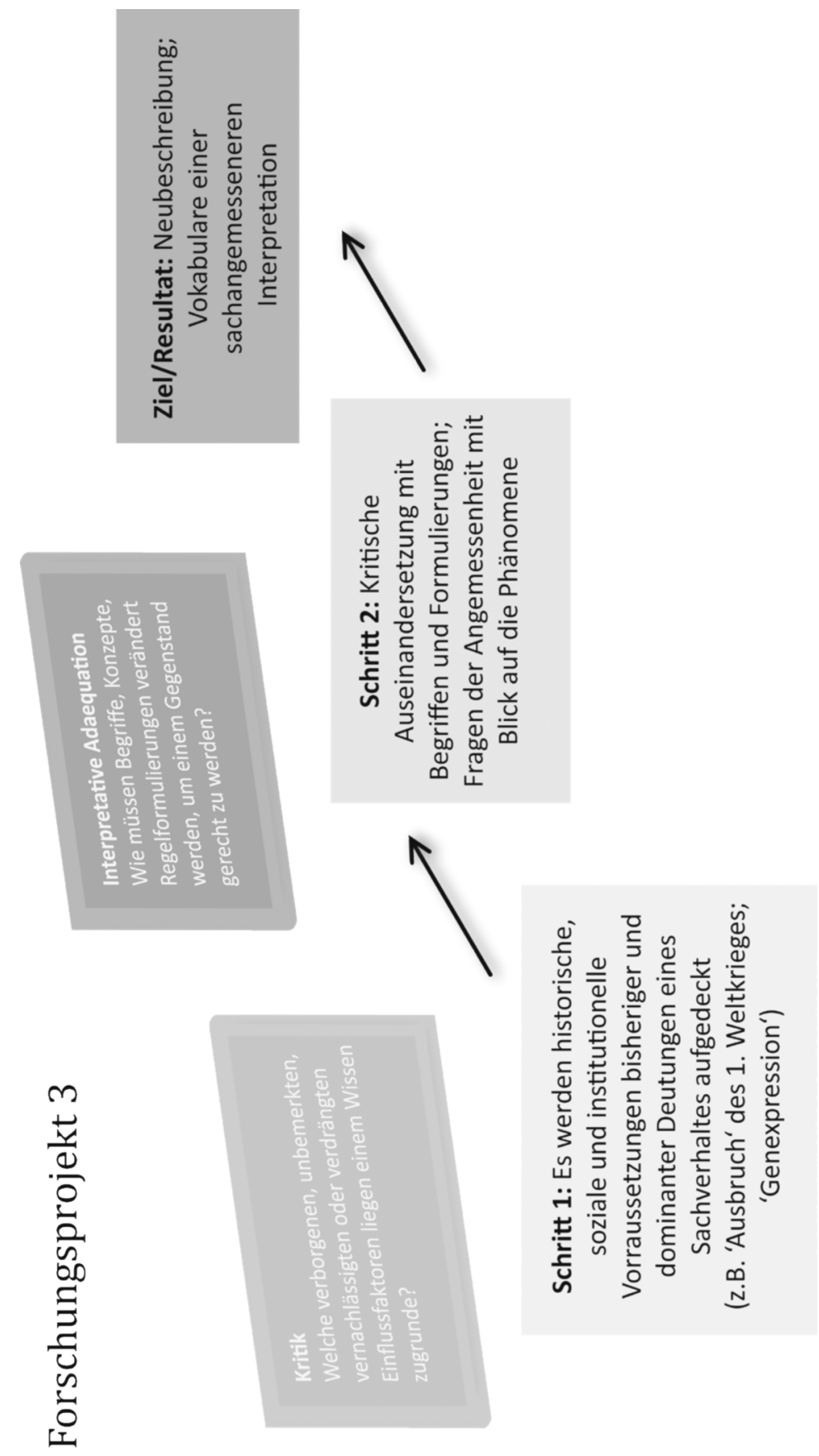


werden, dann lässt sich zielgenau danach fragen, in welchen Disziplinen die geforderten Kompetenzen zu finden sind. Die interdisziplinäre Integration wird hier, anders als bisher, nicht durch eine äußerliche Problemstellung oder durch Strategien der mehr oder weniger verdeckten Reduktion auf spezifische Objektklassen, Theorien oder Methoden vollzogen. Modulare Interdisziplinarität ist radikal nicht reduktiv. Zwar bleibt die Integration in eine übergreifende Leitfragestellung erhalten, doch behalten zum einen Module als komplette Funktionseinheiten ihren Eigenwert und zum anderen lässt sich die Leitfragestellung austauschen. Das heißt, dass die gleiche Forschungsprogrammatik in unterschiedlicher Ausrichtung mehrfach durchlaufen werden kann. Das könnte außerordentlich interessante Ergebnisse liefern und böte zudem die Chance, die Effektivität von Forschungsprogrammen zu erhöhen.

Was in der Logik dieser Überlegungen nicht möglich erscheint, ist eine Forschungsprogrammatik ohne Zielstellung. Ein solch anarchisches Forschen mag als Idee attraktiv erscheinen, ${ }^{15}$ aber es widerspricht der Grundannahme, dass Forschung als Praxis eingebettet in Motivationen arbeitet. Es liegt tief in den historisch gewachsenen Wissenschaftstypen motiviert, was, wie und woraufhin etwas untersucht wird. Motivation bedeutet Ausrichtung. Und wie es zwar immer möglich ist, nacheinander in unterschiedliche

\section{Abb. 18: Aspektwechsel-der Hasen-Enten-Kopf.}

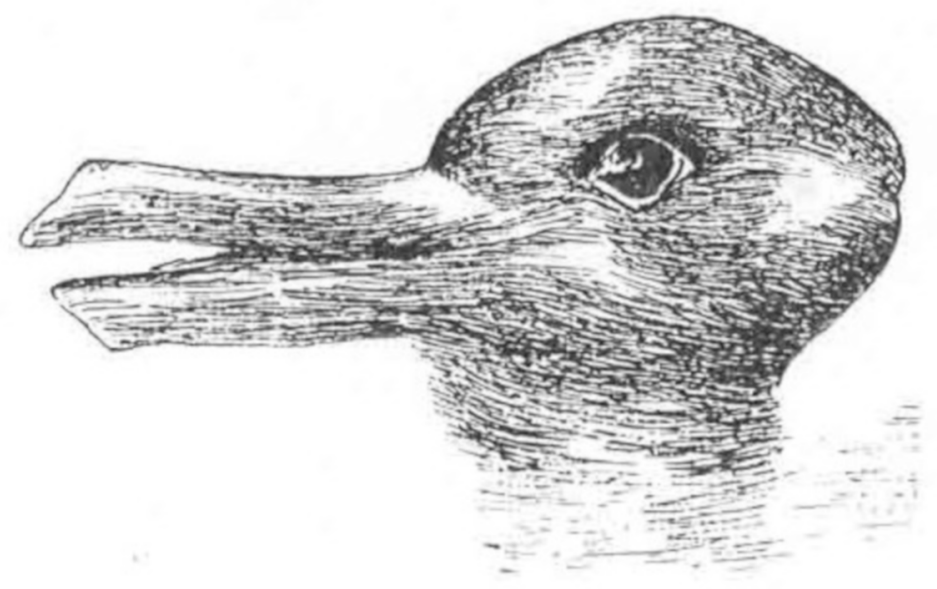

15 Vgl. Paul Feyerabend: Wider den Methodenzwang, Frankfurt a.M. 1983 (1975). 
Richtungen zu gehen und unterschiedliche Ziele anzusteuern, aber nicht zugleich, so bedeutet der modulare Aufbau die Möglichkeit einer alternierenden Zielstellung, nicht aber die Auflösung in ein ranything goes zu jeder Zeit. Wir haben es hier mit der phänomenalen Struktur des Aspektwechsels zu tun. Etwas lässt sich auf die eine und auf andere Arten ansehen; aber nicht zur gleichen Zeit. Die Blickweise kann einmal in der einen Weise ausgerichtet werden, dann wieder in der anderen. Jeweils wird etwas sichtbar, was in der anderen Blickweise verschwindet. Beide Blickweisen betrachten das Ganze, keine ist reduktiv, keine setzt bestimmte Elemente als Letzteinheiten oder bestimmte Strukturen als >die wahre Gestalt‘.

Die Analogie zum visuellen Aspektwechsel trägt noch einen Schritt weiter: Aspektwechsel kann nicht technisch erlernt werden - es gibt dazu keine Methode -; er kann aber geübt werden. ${ }^{16}$ So schult auch eine modulare Form von Interdisziplinarität auf der Tiefenstrukturebene von Wissenschaftstypen eine bestimmte Fähigkeit: Die Fähigkeit, die Blickrichtung zu ändern, bedeutet hier die Kompetenz, Forschung auf verschiedene Weisen anlegen zu können. Auf diese Weise kann erreicht werden, dass Forschungsplanung kreativer und freier und zugleich strukturell tiefer angelegt werden kann.

Spannungen zwischen den Wissenschaftstypen verschwinden auch in diesem modularen Bild wissenschaftlicher Kooperation nicht ganz. Die Auseinandersetzung um Fragen der Art, ob hinter allen Phänomenen eine formal erfassbare Weltordnung steht oder ob jedes individuelle Geschehen eine eigene Ordnung darstellt, die nur rekonstruiert oder beschrieben werden kann; der Streit, ob wir durch alle Bedingtheit unserer Erkenntnisse auf die Realität hindurchsehen können oder ob wir überhaupt nur so viel wahrnehmen, wie wir in seinen Bedingtheiten reflektieren; solche Konflikte werden nicht einfach verschwinden. Doch auch solche Konflikte erscheinen in einem anderen Licht, wenn unterschiedliche Wissenschaftstypen als gleichberechtigt anerkannt werden, und wenn Zusammenarbeit heißt: die unterschiedlichen Typen stark zu machen - stark als eigenständige Module in integrierter Forschung.

16 Vgl. dazu Werner Kogge: Das tätige Auge des Denkens: Aspektwechsel bei Wittgenstein und Fleck. In: Birgit Griesecke (Hrsg.): Werkstätten des Möglichen 1936. L. Fleck, E. Husserl., R. Musil, L. Wittgenstein, Würzburg 2008, S. 59-75. 


\section{Literaturverzeichnis}

Akademie der Wissenschaften zu Berlin (Bernd Grafräth / Huber, Renate/ Uhlemann, Brigitte): Einheit, Interdisziplinarität, Komplementarität. Orientierungsprobleme der Wissenschaft heute, Berlin/New York 1991.

Al-Fārābī: Über die Wissenschaften. De scientiis. Nach der lateinischen Übersetzung Gerhards von Cremona, mit einer Einl. u. kommentierenden Anmerkungen hrsg. und übers. v. Franz Schupp, Hamburg 2005.

Ambrus, Valer: Vom Neopositivismus zur nachanalytischen Philosophie, 2002.

Anscombe, Gertrude E. M.: Causality and Determination. In: Metaphysics and the Philosophy of Mind (The Collected Philosophical Papers: 2), Oxford 1981.

Aristoteles: Metaphysik, Neubearb. d. Übers. v. Hermann Bonitz, hrsg. v. Horst Seidl, Hamburg 1989.

Aristoteles: Über die Seele, auf Grundl. d. Übers. v. W. Theiler hrsg. v. Horst Seidl, Hamburg 1995.

Aristoteles: Nikomachische Ethik, übers. u. hrsg. v. Ursula Wolf, Reinbek bei Hamburg 2006.

Aristoteles: Lehre vom Beweis oder Zweite Analytik (Organon IV), übers., mit einer Einl. u. Anm. hrsg. v. Wolfgang Detel, Hamburg 2011.

Aristoteles-Handbuch. Leben - Werk - Wirkung, hrsg. v. Christof Rapp u. Klaus Corcilius, Stuttgart 2011.

Augustinus: Die christliche Bildung (De doctrina christiana) [426/427], Stuttgart 2002.

Bacon, Francis: Neues Organon, Hamburg 1999.

Bayertz, Kurt: Das Problem der Inkommensurabilität. In: Wissenschaftstheorie und Paradigmabegriff, Stuttgart 1981.

Binder, Harald / Blettner, Maria: Big data in medical science - a biostatistical view. Deutsches Ärzteblatt International 2015, 112, S. 137-42. DOI: 10.3238/ arztebl.2015.0137 
Bloch, Marc: Aus der Werkstatt des Historikers. Zur Theorie und Praxis der Geschichtswissenschaften, Frankfurt/M. u.a. 2000 (im Orig. 1930).

Bock, Henning: Bürgerliche Sammlungen im 18. Jahrhundert. Bildung durch Anschauung. In: Annemarie Gethmann-Siefert/Elisabeth Weisser-Lohmann/Bernadette Collenberg-Plotnikov (Hrsg.): Kunst als Kulturgut. 2, »Kunst« und »Staat«, München 2011, S. 33-50. Open Content Fernuni Hagen: https://www.fernuni-hagen.de/KSW/opencontent/musealisierung/ pdf/Bock_Buergerliche_Sammlungen.pdf

Boden, Margaret A.: What is interdisciplinarity? In: R. Cunningham (Hrsg.): Interdisciplinarity and the organization of knowledge in Europe, Office for Official Publications of the European Communities, Luxembourg 1999.

Boeckh, August: Encyclopädie und Methodologie der philologischen Wissenschaften, hrsg. v. Ernst Bratuscheck, Wiesbaden 1886.

Boethius, Anicius Manlius Severinus: Consolatio philosophiae III, 7. Prosa - 10. Prosa. In: Trost der Philosophie. Übers. u. hrsg. v. Karl Büchner. Mit einer Einf. v. Friedrich Klingner, Stuttgart 1971.

Böhl, Meinrad / Reinhard, Wolfgang / Walter, Peter (Hrsg.): Hermeneutik. Die Geschichte der abendländischen Textauslegung von der Antike bis zur Gegenwart, Wien u.a. 2013, S. 442-548.

Borst, Arno: Das Buch der Naturgeschichte: Plinius und seine Leser im Zeitalter des Pergaments (Abhandlungen der Heidelberger Akademie der Wissenschaften / Philosophisch-historische Klasse), Heidelberg 1994.

Brendecke, Arndt: Information in tabellarischer Disposition. In: Frank Grunert / Anette Syndikus (Hrsg.): Wissensspeicher der Frühen Neuzeit: Formen und Funktionen, Berlin 2015, S. 43-59.

Bruun, Henrik / Hukkinen, Janne / Huutoniemi, Katri / Kleim, Julie Thompson: Promoting Interdisciplinary Research: The Case of the Academy of Finland. Publications of the Academy of Finland, 8 (2005).

Cartwright, Nancy: How the Laws of Physics Lie, New York 1983.

Chladenius, Johann Martin: Einleitung zur richtigen Auslegung vernünftiger Reden und Schriften (Leipzig 1742), Düsseldorf 1969.

Cicero, Marcus Tullius: De re publica. Vom Staat. Übers. u. hrsg. v. Michael von Albrecht, Stuttgart 2013.

Cohen, Benjamin R.: Science and humanities: across two cultures and into science studies. In: Endeaver I (2001), S. 8-12.

Collingwood, Robin G. et al.: Are History and Science Different Kinds of Knowledge? In: Mind 124 (1922), S. 443-466. 
Collini, Stefan: Introduction. In: C. P. Snow: The two Cultures, Cambridge University Press 1998, S. vii-lxxii.

Comte, Auguste: Rede über den Geist des Positivismus, übers., eingel. u. hrsg. v. Iring Fletcher, Hamburg 1994.

Corpus Iuris Civilis. Die Institutionen, übers. v. Okko Behrends, Rolf Knütel, Berthold Kupisch u. Hermann Seiler; hrsg. v. Rolf Knütel, Berthold Kupisch, Sebastian Lohsse u. Thomas Rüfner, Heidelberg u.a. 2013 (4. Aufl.).

Crapanzano, Vincent: Tuhami. Porträt eines Marokkaners, Stuttgart 1983.

Crombie, Alistair Cameron: Commitments and Styles of European Scientific Thinking. History of Science 33 (2) 1995, S. 225-238.

Crombie, Alistair Cameron: Robert Grosseteste and the Origins of Experimental Science. 1100-1700, Oxford 1953.

Crombie, Alistair Cameron: Styles of Scientific Thinking in the European Tradition. A history of argument and explanation especially in the mathematical and biomedical sciences and arts, Bd. 1, London 1994.

d'Alembert, Jean: Abhandlung über Dynamik.; in welcher die Gesetze des Gleichgewichts und der Bewegung der Körper auf die kleinstmögliche Zahl zurückgeführt und in neuer Weise abgeletet werden, und in der ein allgemeines Princip zur Auffindung der Bewegung mehrerer Körper, die in beliebiger Weise aufeinander wirken, gegeben wird. Übers. u. Anm. v. A. Korn, Thun/ Frankfurt/M. 1997 (im Orig. 1743).

Defila, Rico / Di Giulio, Antonietta / Scheuermann, Michael (Hrsg.): Management von Forschungsverbünden - Möglichkeiten der Professionalisierung und Unterstützung, DFG Standpunkte, Weinheim 2008.

Descartes, René: Regulae ad directionem ingenii. Regeln zur Ausrichtung der Erkenntniskraft, krit., revid., übers. u. hrsg. v. Heinrich Springmeyer, Lüder Gäbe, Hans Günter Zekl, Hamburg, 1973.

Descartes, René: Discours de la méthode, Hamburg 1990.

Detel, Wolfgang: Einleitung zu: Aristoteles: Lehre vom Beweis oder Zweite Analytik, Hamburg 2011.

Dilthey, Wilhelm: Einleitung in die Geisteswissenschaften. Versuch einer Grundlegung für das Studium der Gesellschaft und der Geschichte. Bd. 1. Leipzig 1883.

Dilthey, Wilhelm: Der Aufbau der geschichtlichen Welt in den Geisteswissenschaften, Frankfurt/M. 1993 (im Orig. 1910).

Dreyer, Mechthild: More Mathematicorum. Rezeption und Transformation der antiken Gestalten wissenschaftlichen Wissens im 12. Jahrhundert, Münster 1996. 
Droysen, Johann Gustav: Historik. Rekonstruktion der ersten vollständigen Fassung der Vorlesungen (1857), Grundriß der Historik in der ersten handschriftlichen (1857/1858) und in der letzten gedruckten Fassung. Textausgabe v. Peter Leyh, Stuttgart/Bad Cannstatt 1977.

Droysen, Johann Gustav: Beilage: Erhebung der Geschichte zum Rang einer Wissenschaft, In: Ders.: Historik, hrsg. v. Peter Leyh, Stuttgart/Bad Cannstatt 1977, S. 451-469.

Duhem, Pierre: Ziel und Struktur der physikalischen Theorien, Hamburg 1998 (im Orig. 1906).

Eggert, Manfred K. H.: Prähistorische Archöologie. Konzepte und Methoden, Tübingen / Basel 2001.

Esfeld, Michael: Wissenschaft, Erkenntnis und ihre Grenzen. In: Spektrum der Wissenschaft (8) 2017, S. 12-18.

Felt, Ulrike / Nowotny, Helga / Taschwer, Klaus: Wissenschaftsforschung. Eine Einführung, Frankfurt/M. u. a. 1995.

Feyerabend, Paul: Wider den Methodenzwang, Frankfurt/M. 1983 (1975).

Fidora, Alexander: Aristotelische Wissenschaft als Netzwerk von Wissenschaften. Die Rezeption der aristotelischen Wissenschaftstheorie bei alFārābī und Domenicus Gundissalinus. In: Ludger Honnefelder (Hrsg.): Albertus Magnus und der Ursprung der Universitätsidee. Die Begegnung der Wissenschaftskulturen im 13. Jahrhundert und die Entdeckung des Konzepts der Bildung durch Wissenschaft, Weilerswist 2017, S. 77-96.

Fisher, Ronald A.: Statistische Methoden für die Wissenschaft, Edinburgh/London 1956 (12. neu bearb. u. erw. Aufl.) (im Orig. 1925).

Flacius, Matthias Illyricus: De ratione cognoscendi sacras literas. Über den Erkenntnisgrund der Heiligen Schrift, übers., eingel. u. m. Anm. vers. v. Lutz Geldsetzer, [es handelt sich bei dieser Textausgabe um den Anfang des zweiten Teils der Clavis scripturae sacris von 1567, vgl. das Vorwort des Herausgebers], Düsseldorf 1968.

Flasch, Kurt: Geschichte der Philosophie in Text und Darstellung. Mittelalter, Stuttgart 1982.

Fleck, Ludwik: Entstehung und Entwicklung einer wissenschaftlichen Tatsache. Einführung in die Lehre vom Denkstil und Denkkollektiv, Frankfurt/M. 1980.

Fleck, Ludwik: Über die wissenschaftliche Beobachtung und die Wahrnehmung im allgemeinen. In: Ders.: Erfahrung und Tatsache. Gesammelte Aufsätze. Mit einer Einl. hrsg. v. Lothar Schäfer u. Thomas Schnelle, Frankfurt/M. 1983 (1935). 
Foucault, Michel: Die Ordnung der Dinge. Eine Archäologie der Humanwissenschaften [Les mots et les choses], Frankfurt/M. 1971 [1966].

Foucault, Michel: Die Geburt der Biopolitik Geschichte der Gouvernementalität II, Frankfurt/M. 2006.

Frethlöh, Sigrid: Relativismus vs. Universalismus. Zur Kontroverse über Verstehen und Übersetzen in der angelsächsischen Sprachphilosophie: Winch, Wittgenstein, Quine, Aachen 1989.

Fritz, Kurt von: Die Rolle des voṽs. In: Um die Begriffswelt der Vorsokratiker, hrsg. v. Hans Georg Gadamer, Darmstadt 1968.

Fuhrmann, Manfred: Interpretatio. Notizen zur Wortgeschichte. In: Sympotica Franz Wieacker, Göttingen 1970, S. 80-110.

Gähde, Ulrich: Modelle der Struktur und Dynamik wissenschaftlicher Theorien. In: Andreas Bartels/ Manfred Stöckler (Hrsg.): Wissenschaftstheorie, Paderborn 2007.

Galilei, Galileo: Unterredungen und mathematische Demonstrationen über zwei neue Wissenszweige, die Mechanik und die Fallgesetze betreffend, Erster bis sechster Tag, hrsg. von Arthur Oettingen, Darmstadt 1964.

Galilei, Galileo: Dialog über die Weltsysteme. In: Ders., hrsg. u. eingel. v. Hans Blumenberg: Sidereus Nuncius. Nachricht von neuen Sternen, Frankfurt/M. 1965.

Galison, Peter: Die Ontologie des Feindes. Norbert Wiener und die Vision der Kybernetik. In: Michael Hagner (Hrsg.): Ansichten der Wissenschaftsgeschichte, Frankfurt/M. 2001, S. 433-485.

Galton's problem. Wikipedia contributors. (2021, January 1). Galton's problem. In: Wikipedia, The Free Encyclopedia. Retrieved 07:49, June 5, 2021, from https://en.wikipedia.org/w/index.php?title=Galton\%27s_problem \&oldid=997619625

Geertz, Clifford: Dichte Beschreibung. Beiträge zum Verstehen kultureller Systeme, Frankfurt/M. 1991 (im Orig. 1973).

Gehring, Petra / Resch, Michael M. / Kaminski, Andreas (Hrsg.): The Science and Art of Simulation. Exploring - Understanding - Knowing, Heidelberg/ New York 2017.

Geldsetzer, Lutz: Einleitung zu Matthias Flacius Illyricus: De ratione cognoscendi sacras literas / Über den Erkenntnisgrund der Heiligen Schrift, aus der Clavis Scripturae Sacrae. Ausg. von 1719. Instrumenta Philosophica, Series hermeneutica III, Düsseldorf 1968. 
Gigerenzer, Gerd / Swijtink, Zeno / Porter, Theodore / Daston, Lorraine / Beatty, John / Krüger, Lorenz (Hrsg.): Das Reich des Zufalls: Wissen zwischen Wahrscheinlichkeiten, Häufigkeiten und Unschärfen, Heidelberg 1999.

Gigerenzer, Gerd: Das Einmaleins der Skepsis. Über den richtigen Umgang mit Zahlen und Risiken, München/Berlin 2015.

Ginzburg, Carlo: Spurensicherung. Der Jäger entziffert die Fährte, Sherlock Holmes nimmt die Lupe, Freud liest Morelli - Die Wissenschaft auf der Suche nach sich selbst. In: Ders.: Spurensicherung. Über verborgene Geschichte, Kunst und soziales Gedächtnis, München 1988, S. 78-125 (im Orig.: Spie. Radici di un paradigma indiziaro, Torino 1979).

Ginzburg, Carlo: Spuren einer Paradigmengabelung: Machiavelli, Galilei und die Zensur der Gegenreformation. In: Sybille Krämer/ Werner Kogge/ Gernot Grube (Hrsg.): Spur. Spurenlesen als Orientierungstechnik und Wissenskunst, Frankfurt/M. 2007, S. 257-280.

Goodman, Nelson: Ways of Worldmaking, Indianapolis 1978.

Gramelsberger, Gabriele: Simulation and Systems Understanding. In: Hanne Andersen/ Dennis Dieks/ Wenceslao J. Gonzalez/ Thomas Uebel/ Gregory Wheeler (Hrsg.): New Challenges to Philosophy of Science. The Philosophy of Science in a European Perspective, Bd. 4, Dordrecht 2013, S. 151-161.

Griesecke, Birgit / Kogge, Werner: Was ist eigentlich ein Gedankenexperiment? Mach, Wittgenstein und der neue Experimentalismus. In: Marcus Krause/Nicolas Pethes (Hrsg.), Literarische Experimentalkulturen. Poetologien des Experiments im 19. Jahrhundert, Würzburg 2005.

Griesecke, Birgit: Der Innenraum der Erfahrung. Selbstbezug, Selbstobjektivierung und die Genese des Selbstexperiments. In: Nicolas Pethes/ Birgit Griesecke/Marcus Krause/Katja Sabisch: Menschenversuche. Eine Anthologie 1750-2000, Frankfurt/M. 2008.

Griesecke, Birgit: Japan dicht beschreiben. Produktive Fiktionalität in der ethnographischen Forschung, München 2001.

Gundissalinus, Dominicus: De divisione philosophiae. Über die Einteilung der Philosophie. Hrsg., übers., eingel. und mit Anm. vers. von Alexander Fidora u. Dorothée Werner, Freiburg 2007.

Hackett, Jeremiah: Robert Grosseteste and Roger Bacon on the Posterior Analytics. In: Pia Antolic-Piper/Alexander Fidora/Matthias Lutz-Bachmann (Hrsg.): Erkenntnis und Wissenschaft/ Knowledge and Science: Probleme der Epistemologie in der Philosophie des Mittelalters/ Problems of Epistemology in Medieval Philosophy, Berlin 2004, S. 161-212. 
Hacking, Ian: The Accumulation of Styles and Scientific Reasoning. Companion Piece to >Language, Truth and Reason<. In: Steven Lukes/ Martin Hollis: Rationality and Relativism, Oxford 1982.

Hacking, Ian: Einführung in die Philosophie der Naturwissenschaften, Stuttgart 1996 (Orig. 1983 [Representing and Intervening]).

Hacking, Ian: Do Thought Experiments Have a Life of Their Own? Comments on James Brown, Nancy Nersessian and David Gooding. In: PSA: Proceedings of the Biennal Meeting of the Philosophy of Science Association. Vol. Two: Symposia and Invited Papers, Chicago 1992, S. 302-308.

Hacking, Ian: 'Style for Historians and Philosophers. In: Studies in History and Philosophy of Science, 23 (1) 1992, S. 1-20.

Hagner, Michael / Hörl, Erich (Hrsg.): Die Transformation des Humanen, Frankfurt/M. 2008.

Hampe, Michael: Eine kleine Geschichte des Naturgesetzbegriffs, Frankfurt/M. 2007.

Harding, Sandra: Whose Science? Whose Knowledge? Thinking from Women's Lives, Ithaca 1991 (deutsch: Das Geschlecht des Wissens: Frauen denken die Wissenschaft neu, Frankfurt/M. 1994).

Harding, Sandra: »Strong Objectivity«: A Response to the New Objectivity Question. In: Synthese 3 (1995), S. 331-349.

Heidelberger, Michael: Die Erweiterung der Wirklichkeit im Experiment. In: Michael Heidelberger/Friedrich Steinle (Hrsg.): Experimental Essays = Versuche zum Experiment, Baden Baden 1998, S. 71-92.

Heidelberger, Michael: Das Experiment und die Wissenschaften. In: Wissenschaftstheorie. Ein Studienbuch, Paderborn 2007, S. 155-176.

Henrich, Dieter (Hrsg.): Kant oder Hegel? Über Formen der Begründung in der Philosophie, Stuttgart 1983, S. 453-465.

Hertz, Heinrich: Die Prinzipien der Mechanik, Leipzig 1894.

Hilbert, David: >Axiomatisches Denken< (1918). In: David Hilbert: Hilbertiana. FünfAufsätze von David Hilbert, Darmstadt 1964, S. 1-11.

Hobbes, Thomas: Leviathan, Frankfurt/M. 1994.

Höffe, Otfried: Einführung in die Wissenschaftstheorie der Zweiten Analytik. In: Aristoteles: Lehre vom Beweis oder Zweite Analytik (Organon IV), übers. u. m. Anm. vers. v. Eugen Rolfes; m. neuer Einl. u. Bibliographie v. Otfried Höffe, Hamburg 1990, S. VII-XLII. 
Horstmann, Axel: Das Fremde und das Eigene - >Assimilation< als hermeneutischer Begriff. In: Archiv für Begriffsgeschichte, Bd. 30, Bonn 1986/87, S. 7-43.

Hoyningen-Huene, Paul: Die Wissenschaftsphilosophie des Thomas S. Kuhns. Rekonstruktion und Grundlagenprobleme, Braunschweig 1989.

Hoyningen-Huene, Paul: Inkommensurabilität bei Kuhn und Theorienvergleich In: E. Agazzi (Hrsg.): Die Vergleichbarkeit wissenschaftlicher Theorien, Freiburg 1990.

Husserl, Edmund: Ideen zu einer reinen Phänomenologie und phänomenologischen Philosophie (1913), Ges. Schriften Bd. 5, hrsg. v. Elisabeth Ströker, Hamburg 1992, (Husserliana Bd. III,1, V).

Hüttemann, Andreas: Naturgesetze. In: Andreas Bartels/ Manfred, Stöckler (Hrsg.): Wissenschaftstheorie, Paderborn 2007, S. 135-154.

Ihde, Don: Instrumental Realism: The Interface between Philosophy of Science and Philosophy of Technology, Bloomington/Indianapolis 1991.

Jansen, Martin / Wedig, Ulrich: Das Teil im Ganzen - Missverständnisse um chemische Konzepte. In: Angewandte Chemie 2008, 120, S. 10176-10180.

Jung, Matthias / Moritz, Arne / Schlette, Magnus: Philosophie. In: Meinrad Böhl/ Wolfgang Reinhard/Peter Walter: Hermeneutik. Die Geschichte der abendländischen Textauslegung von der Antike bis zur Gegenwart, Wien u.a. 2013, S. 442-548.

Kälble, Hartmut / Schriewer, Jürgen (Hrsg.): Vergleich und Transfer. Komparatistik in den Sozial-, Geschichts- und Kulturwissenschaften, Frankfurt/M. u.a. 2003.

Kant, Immanuel: Der Streit der Fakultäten (1798). In: Ders.: Schriften zur Anthropologie, Geschichtsphilosophie, Politik und Pädagogik 1, Werkausgabe Bd. XI, hrsg. v. Wilhelm Weischedel, Frankfurt/M. 1977, S. 261-393.

Kant, Immanuel: Kritik der reinen Vernunft 2. Werkausgabe Bd. 4, hrsg. v. Wilhelm Weischedel, Frankfurt/M. 1974.

Kay, Lily E.: Das Buch des Lebens: Wer schrieb den genetischen Code?, München 2002.

Keller, Evelyn Fox: Das Leben neu Denken. Metaphern der Biologie im 20. Jahrhundert, München 1998.

Kirchhoff, Gustav: Vorlesungen über mathematische Physik. Mechanik, Leipzig 1876. 
Klein, Julie Thompson: A taxonomy of interdisciplinarity. In: Robert Frodeman/ Julie Thompson Klein/ Carl Mitcham/ J. Brott Holbrook (Hrsg.): The Oxford Handbook of Interdisciplinarity, Oxford 2010, S. 15-30.

Klein, Ursula: Experiments, Models, Papertools. Cultures of Organic Chemistry in the Nineteenth Century, Stanford 2003.

Klipp, Edda / Herwig, Ralf / Kowald, Axel / Wierling, Christoph / Lehrach, Hans (Hrsg.): Systems Biology in Practice. Concepts, Implementation and Application, Weinheim 2004.

Knorr-Cetina, Karin: Die Fabrikation von Erkenntnis. Zur Anthropologie der Wissenschaft, Frankfurt/M. 1987.

Kogge, Werner: Verstehen und Fremdheit in der philosophischen Hermeneutik: Heidegger und Gadamer, Hildesheim 2001.

Kogge, Werner: Die Grenzen des Verstehens. Kultur - Differenz-Diskretion, Weilerswist 2002.

Kogge, Werner: Das tätige Auge des Denkens: Aspektwechsel bei Wittgenstein und Fleck. In: Birgit Griesecke (Hrsg.): Werkstätten des Möglichen 1936. L. Fleck, E. Husserl., R. Musil, L. Wittgenstein, Würzburg 2008, S. 59-75.

Kogge, Werner: Technologie des 21. Jahrhunderts. Perspektiven der Technikphilosophie. In: Deutsche Zeitschrift für Philosophie, 6 (2008), S. 935-956.

Kogge, Werner / Griesecke, Birgit: Ein Arbeitsprogramm, kein Abgesang. Wittgensteins grammatische Methode als Verfahren experimentellen Denkens. In: Stefan Tolksdorf/Holm Tetens: In Sprachspiele verstrickt, oder, Wie man der Fliege den Ausweg zeigt: Verflechtungen von Wissen und Können, Berlin/New York 2010, S. 101-126.

Köhler, Theodor W.: Sachverhaltsbeobachtung und axiomatische Vorgaben. Zur Struktur wissenschaftlicher Erfassung konkreter Äußerungsweisen des Menschlichen im 13. Jahrhundert. In: Pia Antolic-Piper/ Alexander Fidora/ Matthias Lutz-Bachmann (Hrsg.): Erkenntnis und Wissenschaft/ Knowledge and Science: Probleme der Epistemologie in der Philosophie des Mittelalters/ Problems of Epistemology in Medieval Philosophy, Berlin 2004, S. 125-150.

Krämer, Sybille: Symbolische Maschinen. Die Geschichte der Formalisierung im historischen Abriß, Darmstadt 1988.

Kuhn, Thomas S.: Die Entstehung des Neuen: Studien zur Struktur der Wissenschaftsgeschichte, Frankfurt/M. 1984.

Kuhn, Thomas S.: Die Struktur wissenschaftlicher Revolutionen, Frankfurt/M. 1988 (1962). 
Kuhn, Thomas S.: Postskriptum. In: Ders.: Die Struktur wissenschaftlicher Revolutionen, Frankfurt a.M. 1988, S. 186-221.

Kurz, Gerhard: Hermeneutische Künste. Die Praxis der Interpretation, Stuttgart 2018.

Lambdin, Charles: Significance tests as sorcery: Science is empirical - significance tests are not. In: Theory \& Psychology 22,1 (2012), S. 67-90.

Lao, Eugenia: Taxonomic Organization in Pliny's Natural History. In: Papers of the Langford Latin Seminar, 16 (2016), S. 209-246.

Latour, Bruno: Science in Action: How to Follow Scientists and Engineers through Society, Cambridge MA 1987.

Ledford, Heidi: Team Science. In: Nature, 525, 7569 (2015), S. 308-311.

Lepenies, Wolf: Das Ende der Naturgeschichte: Wandel kultureller Selbstverständlichkeiten in den Wissenschaften des 18. und 19. Jahrhunderts, München 1976.

Lévi-Strauss, Claude: Das Ende des Totemismus, Frankfurt/M. 1965.

Lévi-Strauss, Claude: Das wilde Denken, Frankfurt/M. 1973.

Locke, John: Versuch über den menschlichen Verstand, Hamburg 1988.

Mach, Ernst: Die Mechanik in ihrer Entwicklung. Historisch-kritisch dargestellt. Hrsg. u. m. einem Anh. vers. v. Renate Wahsner u. Horst-Heino von Borzeszkowski, Berlin 1988 (1883).

Mach, Ernst: Über Gedankenexperimente. In Ders.: Erkenntnis und Irrtum. Skizzen zur Psychologie der Forschung, Leipzig 1905, S. 183-201.

Mach, Ernst: Die Vergleichung als wissenschaftliches Princip. In: Die Principien der Wärmelehre. Historisch-kritisch entwickelt von Ernst Mach, Leipzig 1896 (3. Aufl. 1919), S. 396-405.

Mante, Gabriele: Spuren lesen. Die Relevanz kriminalistischer Methoden für die archäologische Wissenschaft. In: Ulrich Veit/Tobias L. Kienlin/ Christoph Kümmel/ Sascha Schmidt (Hrsg.): Spuren und Botschaften. Interpretationen materieller Kultur. New York/München/Berlin 2003, S. 157-172.

Matthes, Joachim: The Operation Called >Vergleichen<. In: Ders.: Zwischen den Kulturen? Die Sozialwissenschaften vor dem Problem des Kulturvergleichs, (Soziale Welt Sonderband 8), Göttingen 1992, S. 75-102.

Merton, Robert K.: Die normative Struktur von Wissenschaft. In: Ders.: Entwicklung und Wandel von Forschungsinteressen. Aufsätze zur Wissenschaftssoziologie, Frankfurt/M. 1995 (1942), S. 86-99.

Mill, John Stuart: System der deduktiven und induktiven Logik. Eine Darlegung der Prinzipien wissenschaftlicher Forschung, insbesondere der Naturforschung, o. O. 2017. 
Misch, Georg: Zur Entstehung des französischen Positivismus, Darmstadt 1969.

Mitchell, Sandra D.: Ceteris Paribus: An Inadequate Representation for Biological Contingency. In: Erkenntnis. An International Journal of Analytic Philosophy, 57 (2002), S. 329-350.

Nuzzo, Regina: Fehlerschätzung. Der Fluch des P-Wertes. In: Spektrum der Wissenschaft, Spezial (3) 2017 (zunächst in Heft 9/ 2014).

Oppenheim, Paul / Putnam, Hilary: Unity of science as a working hypothesis. In: Herbert Feigl (Hrsg.): Concepts, theories and the mind-body problem. Minnesota studies in the philosophy of science. Minneapolis: University of Minnesota Press, 1958, S. 3-36.

Pickering, Andrew: The Mangle of Practice: Time, Agency, and Science, Chicago 1995.

Planck, Max: Die Einheit des physikalischen Weltbildes. Vortrag, gehalten am 9. Dezember 1908 in der naturwissenschaftlichen Fakultät des Studentenkorps an der Universität Leiden, Leipzig 1909.

Platon: Politikos. Übers. v. Friedrich Schleiermacher. Sämtliche Werke in zehn Bänden, Frankfurt/M. u. Leipzig 1991.

Plinius, Secundus Gaius: Naturkunde (lateinisch - deutsch), hrsg. u. übers. v. Roderich König in Zus. m. Joachim Hopp u. Wolfgang Glöckner, Düsseldorf/ Zürich 1997 (2. Aufl.).

Polanyi, Michael: Implizites Wissen. Frankfurt/M. 1985 [1966].

Pollmann, Karla: Nachwort. In: Aurelius Augustinus: Die christliche Bildung (De doctrina christiana). Übersetzung, Anmerkung und Nachwort v. Karla Pollmann, Stuttgart 2013.

Quételet, Adolphe: Soziale Physik oder Abhandlung über die Entwicklung der Fähigkeiten des Menschen; n. d. Ausg. letzter Hand (1869) übers. v. Valentine Dorn u. eingel. v. Heinrich Waentig, 1. Bd., Jena 1914.

Ranke, Leopold von: Englische Geschichte vornehmlich im siebzehnten Jahrhundert. Zweiter Band. In: Sämmtliche Werke Band 15, Leipzig 1870.

Rheinberger, Hans-Jörg: Experiment. Differenz. Schrift: Zur Geschichte epistemischer Dinge, Marburg an der Lahn 1992.

Rheinberger, Hans-Jörg: Experimentalsysteme und epistemische Dinge. Eine Geschichte der Proteinsynthese im Reagenzglas, Göttingen 2001.

Rheinberger, Hans-Jörg: Epistemologica: Präparate. In: Anke von Heesen/ Petra Lutz (Hrsg.): Dingwelten. Das Museum als Erkenntnisort. Schriften des deutschen Hygiene-Museums Dresden, Köln/Weimar/Wien 2005, S. 65-76. 
Roesner, Martina: Amor Scientiae und Studium generale. Die Geburt der Idee der Universität im 12./ 13. Jahrhundert. In: Ludger Honnefelder (Hrsg.): Albertus Magnus und der Ursprung der Universitätsidee. Die Begegnung der Wissenschaftskulturen im 13. Jahrhundert und die Entdeckung des Konzepts der Bildung durch Wissenschaft, Weilerswist 2017, S. 51-76.

Rouse, Joseph: Engaging Science: How to Understand its Practices Philosophically, Ithaca/Cornell 1996.

Saint-Mont, Uwe: Statistik im Forschungsprozess. Eine Philosophie der Statistik als Baustein einer integrativen Wissenschaftstheorie, Heidelberg u.a. 2011.

Sarnowsky, Jürgen: Expertus - experientia - experimentum. Neue Wege der wissenschaftlichen Erkenntnis im Spätmittelalter. In: Das Mittelalter 17, 2: 47-59. doi: https://doi.org/10.1524/mial.2012.0021.

Saussure, Ferdinand de: Grundlagen der allgemeinen Sprachwissenschaft, Berlin 1967 (1906-1911).

Scherpe, Klaus R.: Beschreiben, Nicht Erzählen! Beispiele Zu Einer Ästhetischen Opposition. In: Zeitschrift für Germanistik, 6 (2) 1996, S. 368-383.

Schleiermacher, Friedrich: Über den Begriff der Hermeneutik mit Bezug auf F. A. Wolfs Andeutungen und Asts Lehrbuch. In: Hermeneutik und Kritik, hrsg. u. eingel. v. Manfred Frank, S. 309-346.

Schmidt, Jan C.: Towards a philosophy of interdisciplinarity. An attempt to provide a classification and clarification. In: Poiesis and Praxis 5 (2008), S. 53-69.

Schmidt, Jan C.: What is a problem? On problem-oriented interdisciplinarity, Poiesis and Praxis 4 (2011), S. 249-274.

Schupp, Franz: Einleitung. In: Al-Fārābī: Über die Wissenschaften. De scientiis. Nach der lateinischen Übersetzung Gerhards von Cremona, mit einer Einl. u. kommentierenden Anmerkungen hrsg. und übers. v. Franz Schupp, Hamburg 2005.

Schurz, Gerhard: Wissenschaftliche Erklärung. In: Wissenschaftstheorie. Ein Studienbuch, hrsg. v. Andreas Bartels u. Manfred Stöckler, Paderborn 2007, S. 69-88.

Sokal, Alan / Bricmont, Jean: Eleganter Unsinn. Wie die Denker der Postmoderne die Wissenschaften mißbrauchen, München 1999.

Speer, Andreas: Scientia demonstrativa et universaliter ars faciens scire. Zur methodischen Grundlegung einer Wissenschaft von der Natur durch Robert Grosseste. In: Erfahrung und Beweis. Experience and Demonstration, 
hrsg. v. Alexander Fidora u. Matthias Lutz-Bachmann, Berlin 2007, S. 25-40.

Spektrum der Wissenschaft. Physik, Mathematik, Technik, Spezial: Vorsicht Statistik! Vom Gesetz der großen Zahlen bis zu Klimarekorden, Heft 3 (2017).

Stadler, Friedrich: Studien zum Wiener Kreis. Ursprung, Entwicklung und Wirkung des Logischen Empirismus im Kontext, Frankfurt/M. 1997.

Sterman, J. D.: A Skeptic's Guide to Computer Models. In: G. O. Barneyet et al. (Hrsg.): Managing a Nation: The Microcomputer Software Catalog. Boulder, CO 1991, S. 209-229.

Sticker, Bernhard: Alexander von Humboldt und die Einheit der Wissenschaft. Studia Leibnitiana, Bd. 2, H. 4 (1970), S. 241-261.

»Systema Naturae«. In: Wikipedia - Die freie Enzyklopädie. Bearbeitungsstand: 16. Juli 2021, 19:37 UTC. URL: https://de.wikipedia.org/w/index. php?title=Systema_Naturae\&oldid=213940312 (Abgerufen: 9. November 2021, 18:48 UTC)

Szabo, Árpád: Anfänge der griechischen Mathematik, Wien 1969.

Tetens, Holm: Experimentelle Erfahrung, Hamburg 1987.

Thieme, Hartmut (Hrsg.): Die Schöninger Speere - Mensch und Jagd vor 400.000 Jahren, Stuttgart 2007.

Toulmin, Stephen I.: Einführung in die Philosophie der Wissenschaft, Göttingen 1969 (im Orig. 1953).

Toulmin, Stephen: Voraussicht und Verstehen. Ein Versuch über die Ziele der Wissenschaft, Frankfurt/M. 1968.

Verein Ernst Mach (Hrsg.): Wissenschaftliche Weltauffassung. Der Wiener Kreis, Wien 1929.

Wasserstein, Ronald L. / Lazar, Nicole A.: The ASA Statement on p-Values: Context, Process, and Purpose. In: The American Statistician, 2 (2016), S. 129-133.

West, David M. / Teravis, Larry E.: From Society to Landscape. Alternative Metaphors für Artificial Intelligence. In: AI Magazine, 12 (2) 1991; im Orig.: .

Wilholt, Torsten: Zahl und Wirklichkeit. Eine philosophische Untersuchung über die Anwendbarkeit der Mathematik, Paderborn 2004.

Winch, Peter: The Idea of a Social Science, London 1958.

Windelband, Wilhelm: Geschichte und Naturwissenschaft, Straßburger Rektoratsrede 1894, Straßburg 1904. 
Wittgenstein, Ludwig: Bemerkungen über die Grundlagen der Mathematik, Werkausgabe Bd. 6, Frankfurt/M. 1984.

Wittgenstein, Ludwig: Das Blaue Buch. Werkausgabe Bd. 5, Frankfurt/M 1984. Wittgenstein, Ludwig: Philosophische Untersuchungen. Werkausgabe Bd. I, Frankfurt/M. 1982.

Wright, Georg H. von: Erklären und Verstehen, Frankfurt/M. 1993 (3. Aufl. (im Orig. 1971)).

Wright, Georg H. von: Laws of Nature. In: Truth, Knowledge and Modality, Philosophical Papers 3 (1984), S. 135-149.

\section{Lexika}

Etymologisches Wörterbuch des Deutschen. Erarbeitet von einem Autorenkollektiv des Zentralinstituts für Sprachwissenschaft und der Leitung von Wolfgang Pfeifer, Berlin 1989.

Historisches Wörterbuch der Philosophie, hrsg. v. Joachim Ritter/ Karlfried Gründer/ Gottfried Gabriel, Basel 1971-2007.

Lexikon der Sprachwissenschaften. Hrsg. v. Hadumod Bussman, Stuttgart 2008. Routledge Encyclopedia of Philosophy. Hrsg. v. Edward Craig, London/New York 1998. 


\section{Abbildungsverzeichnis}

Abb. 1: Die Zeichnung befindet sich im zitierten Text auf S. 3. Euklid: Die Elemente. Buch I-VIII. Nach Heibergs Text aus dem Griechischen übersetzt und herausgegeben von Clemens Thaer, Darmstadt 1973.

Abb. 2: Aus Steven Toulmin: Einführung in die Philosophie der Wissenschaft, Göttingen 1969 (im Orig. 1953), S. 26.

Abb. 3: Aus Adolphe Quételet: Soziale Physik oder Abhandlung über die Entwicklung der Fähigkeiten des Menschen; n. d. Ausg. letzter Hand (1869) [im Orig. 1835] übers. v. Valentine Dorn u. eingel. v. Heinrich Waentig, 1. Bd., Jena 1914, S. 106.

Abb. 4: Aus Tyler Vigen: Spurious Correlations. Correlation does not equal causation, New York 2015, S. 61.

Abb. 5: Aus Adolphe Quételet: Soziale Physik oder Abhandlung über die Entwicklung der Fähigkeiten des Menschen; n. d. Ausg. letzter Hand (1869) [im Orig. 1835] übers. v. Valentine Dorn u. eingel. v. Heinrich Waentig, 1. Bd., Jena 1914, S. 145.

Abb. 6: Aus Adolphe Quételet: Soziale Physik oder Abhandlung über die Entwicklung der Fähigkeiten des Menschen; n. d. Ausg. letzter Hand (1869) [im Orig. 1835] übers. v. Valentine Dorn u. eingel. v. Heinrich Waentig, 1. Bd., Jena 1914, S. 146.

Abb. 7: Aus Ronald A. Fisher: Statistische Methoden für die Wissenschaft, Edinburg/London 1956 (12. neu bearb. u. erw. Aufl.) (im Orig. 1925), S. 31.

Abb. 8: Darstellung des p-Werts aus: Wikipedia-Commons: User: Repapetilto @ Wikipedia \& User:Chen-Pan Liao @Wikipedia, CC BY-SA 3.o, https:// creativecommons.org/licenses/by-sa/3.0, via Wikimedia Commons.

Abb. 9: Laerd Statistics - https://statistics.laerd.com/statistical-guides/ pearson-correlation-coefficient-statistical-guide.php, CC BY-SA 4.0, https://commons.wikimedia.org/w/index.php?curid=84255641. 
Abb. 10: Aus Auguste Comte: Cours de Philosophie Positive, 1. Band. Tableau Synoptique, Paris 1949, (nach S. LII).

Abb. 11: Paul Oppenheim/Hilary Putnam: The Unity of Science as a Working Hypothesis. In: Minnesota Studies in the Philosophy of Science, 1958, S. 3-35; die graphische Darstellung der »levels« befindet sich auf S. 9.

Abb. 12: Synopse von Wissenschaftstypen und ihrer Ausprägungen. (c) bei Werner Kogge.

Abb. 13: Wissenschaftstypen in nicht-serieller Darstellung. (c) bei Werner Kogge.

Abb. 14: Verlaufsdiagramm typischer wissenschaftlicher Arbeitsschritte. (c) bei Werner Kogge.

Abb. 15: Darstellung der Varianz möglicher Verläufe im wissenschaftlichen Arbeiten. (c) bei Werner Kogge.

Abb. 16: Modulare Darstellung von Wissenschaftstypen. (c) bei Werner Kogge. Abb. 17: Drei zu Anschauungszwecken stark vereinfachte Beispiele modularer Kombination von Forschungstypen. (c) bei Werner Kogge.

Abb. 18: Hasen-Enten-Kopf. Abb. aus: Public Domain, https://commons.wikimedia.org/w/index.php?curid=667017. 


\section{Namensregister}

A

Al-Fārābī 19, 134-135

Ambrus, Valer 58

Ampère, André-Marie 66

Anscombe, Gertrude E. M. 67

Aquin, Thomas von 56, 77

Aristoteles 18-25, 31, 41-46, 56-57, 72-73, 77, 81, 87, 94-96, 105, 109, 114,133-137, 155,167

Arnold, Matthew 30

Augustinus 18-20, 32-35, 74, 83,

B

Bachelard, Gaston 100

Bacon, Francis 32, 52-54, 60, 137-139

Bacon, Roger 44, 56

Bayertz, Kurt 185

Beatty, John 119

Binder, Harald 128

Blettner, Maria 128

Bloch, Marc 110

Bock, Henning 38-39

Boden, Margaret A. 185

Boeckh, August 78

Boethius, Anicius Manlius Severinus 80-84, 87

Böhl, Meinrad 31-32, 70, 77

Borst, Arno 37 
Brendecke, Arndt 39
Bricmont, Jean 160
Bruun, Henrik 185
Buffon, Louis Leclerc 38
Bussman, Hadumod 103

C

Cartwright, Nancy 153, 67-68

Chladenius, Johann Martin 89-90

Chomsky, Noam 103

Cicero, Marcus Tullius 32, 87

Cohen, Benjamin R. 29

Collingwood, Robin G. 150-151

Collini, Stefan 29

Comte, Auguste 140-143

Corcilius, Klaus 42

Crapanzano, Vincent 79

Cremona, Gerhard von 19, 134-135

Crombie, Alistair Cameron 44, 165-169

D

d'Alembert, Jean 98, 138,

Darwin, Charles 29

Daston, Lorraine 85, 119

Defila, Rico 180-182

Descartes, René 14, 25-26, 45, 53-55, 73-74, 137-138, 154-155

Detel, Wolfgang 24-25

Di Giulio, Antonietta 182

Dilthey, Wilhelm 80, 145-147, 154

Dreyer, Mechthild 20, 43

Droysen, Johann Gustav 27-28, 33, 70-71, 74-78, 89, 143-147, 164

Duhem, Pierre 66, 69

E

Eggert, Manfred K. H 105

Esfeld, Michael 55

Euklid 44-47, 72 


\section{F}

Felt, Ulrike 158-159

Feyerabend, Paul 160, 201

Fidora, Alexander 19, 44, 135-137

Fisher, Ronald A. 118, 122-124

Flacius, Matthias Illyricus 34-35

Flasch, Kurt 81-82

Fleck, Ludwik 90, 95, 151-154, 202

Foucault, Michel 39, 90-91, 154, 157

Frethlöh, Sigrid 109

Fritz, Kurt von 26

Fuhrmann, Manfred 31

\section{G}

Gähde, Ulrich 68

Galilei, Galileo 27, 45, 52-54, 137-138, 164

Galison, Peter 85, 189

Galton, Francis 106

Gauß, Carl Friedrich 121

Geertz, Clifford 33, 78-79, 99-100

Gehring, Petra 62, 113

Geldsetzer, Lutz 34-35

Gigerenzer, Gerd 111, 119

Ginzburg, Carlo 75, 163-164

Goodman, Nelson 154-155

Grafräth, Bernd 14

Gramelsberger, Gabriele 62, 73

Griesecke, Birgit 79, 83, 94, 150, 202

Grosseteste, Robert 44, 56

Gundissalinus, Dominicus 19, 135-136

\section{H}

Hackett, Jeremiah 44

Hacking, Ian 58-59, 153, 166

Hagner, Michael 187

Hampe, Michael 55, 64-65

Harding, Sandra 85-86 
Heidelberger, Michael 52-53, 58

Henrich, Dieter 166

Herder, Johann G. 15

Hertz, Heinrich 97-98

Herwig, Ralf 61

Hilbert, David 41, 154-155

Hobbes, Thomas 26, 89, 96

Höffe, Otfried 17

Holmes, Sherlock 60, 75

Hörl, Erich 188

Horstmann, Axel 77-78

Hoyningen-Huene, Paul 76, 182

Huber, Renate 14

Hukkinen, Janne 185

Humboldt, Alexander von 15, 38

Hume, David 29, 88

Husserl, Edmund 92, 202

Hüttemann, Andreas 63, 65

Huutoniemi, Katri 185

Huxley, Thomas H. 29

\section{I}

Ihde, Don 59, 153

Iustitian 32

J

Jansen, Martin 92

Jung, Matthias 77

$\mathbf{K}$

Kälble, Hartmut 106

Kaminski, Andreas 62

Kant, Immanuel 14, 73, 88, 139-140, 146, 155, 166

Kay, Lily E. 187

Keller, Evelyn Fox 91, 187

Kepler, Johannes 27, 64, 66

Kirchhoff, Gustav 96 
Klein, Julie Thompson 185

Klein, Ursula 66-67

Klipp, Edda 61

Knorr-Cetina, Karin 15

Knütel, Rolf 32

Kogge, Werner 73, 79, 83, 94, 109, 164, 186, 202

Köhler, Theodor W. 56-57

Kowald, Axel 61

Krämer, Sybille 49, 164

Krüger, Lorenz 119

Kues, Nikolaus von 77

Kuhn, Thomas S. 58, 67-68, 76, 90, 152, 157, 160, 182

Kupisch, Berthold 32

Kurz, Gerhard 32

\section{L}

Lambdin, Charles 111

Lao, Eugenia 39

Latour, Bruno 59, 154

Lazar, Nicole A. 126

Ledford, Heidi 187

Lehrach, Hans 61

Leibniz, Gottfried W. 14, 119, 138

Lepenies, Wolf 39

Lévi-Strauss, Claude 108,

Linné, Carl von 38, 109

Locke, John 88

Lohsse, Sebastian 32

M

Mach, Ernst 66, 97-98, 147-150

Malinowski, Bronislaw 78

Mante, Gabriele 99

Matthes, Joachim 103

Maturana, Humberto 91, 154

Merton, Robert K. 159

Mill, John Stuart 140-143, 168 
Misch, Georg 97-98

Mitchell, Sandra D. 63

Moritz, Arne 77

Morley, Daniel von 19

\section{$\mathbf{N}$}

Newton, Isaac 27, 64, 66, 142

Nowotny, Helga 158-159

Nuzzo, Regina 128

\section{O}

Oppenheim, Paul 15, 24, 148-149

$\mathbf{P}$

Pfeifer, Wolfgang 21

Pickering, Andrew 59, 153

Planck, Max 15

Platon 20, 77, 81, 87, 104-105, 154-155, 167

Plinius, Secundus Gaius 36-38

Poincaré, Henri 66

Polanyi, Michael 95, 152

Pollmann, Karla 32

Porphyrios 77

Porter, Theodore 119

Putnam, Hilary 15, 148-149

Q

Quételet, Adolphe 113-121

Quintilian 87

$\mathbf{R}$

Ramus, Petrus 25, 87-88

Ranke, Leopold von 78

Rapp, Christof 42

Reinhard, Wolfgang 31, 70, 77

Resch, Michael M. 62

Rheinberger, Hans-Jörg 59-60, 100-101, 153 
Roesner, Martina 18-21

Rouse, Joseph 59, 153

Rüfner, Thomas 32

\section{S}

Saint-Mont, Uwe 111-112

Sarnowsky, Jürgen 44

Saussure, Ferdinand de 107-108

Scherpe, Klaus R. 100

Scheuermann, Michael 180

Schleiermacher, Friedrich 33, 35, 70, 74, 77, 87, 90

Schlette, Magnus 77

Schmidt, Jan C. 185-186

Schriewer, Jürgen 106

Schupp, Franz 19, 134-136

Schurz, Gerhard 24-25

Seiler, Hermann 29

Snow, Charles P. 160

Sokal, Alan 160

Speer, Andreas 44

Stadler, Friedrich 148

Sterman, J. D. 62

Sticker, Bernhard 15

Swijtink, Zeno 119

Szabo, Árpád 42

$\mathrm{T}$

Taschwer, Klaus 158-159

Teravis, Larry E. 92

Tetens, Holm 59, 67, 94, 153

Thieme, Hartmut 172

Toulmin, Stephen 49-51, 128, 162-163

Tylor, Edward 106

U

Uhlemann, Brigitte 14 
226 Einführung in die Wissenschaften

V

Varela, Francisco 91, 154, 188

W

Walter, Peter 70, 77

Wasserstein, Ronald L. 126

Wedig, Ulrich 92

West, David M. 92

Wierling, Christoph 61

Wilholt, Torsten 49

Winch, Peter 108-109

Windelband, Wilhelm 28, 146-147

Wittgenstein, Ludwig 46, 94, 109, 148-155, 171, 202

Wright, Georg H. 67, 171 


\section{Sachregister}

A

Ähnlichkeit 56, 110, 138, 153, 171

Allgemeinheit; Verallgemeinerung 28, 50, 64, 65, 68, 75, 99, 140, 164

Analyse; analytisch 13, 40, 69, 71-74, 78, 87, 144, 165, 167-168, 183, 192

anpassen; Anpassung 33, 76-79, 94

Anthropologie 36-37, 78, 108, 172, 187

Antike 15, 21-22, 33-36, 42-43, 64, 80, 88, 186

Astronomie 18, 33, 36, 41, 54, 128, 135, 140, 142

Atomismus 188, 55

auslegen; Auslegung 31-32, 74, 89, 137, 176, 178

Axiom; Axiomatik 20, 41-46, 137, 154

\section{B}

Bedingungen, Bedingungsgefüge 29, 48-49, 60, 65, 71, 86-93, 99, 122, 127, 147, $153,155,160-162,172,175-177,192-195$

Begriffsgeschichte 46,80

beobachten; Beobachtung 52-57, 60, 65, 92-93, 96, 100-101, 118-119, 128, 148, $151,158,163-164,174,177-178$

beschreiben; Beschreibung; description 36, 39, 51, 64-65, 76-79, 92-100, 115, 136, 146, 167, 172, 176-177, 179-180, 183, 191, 194

Beweis; Beweislehre; Beweisführung; apodeixis; demonstratio 17-20, 24-27, 36, $41,45-49,53-64,72-74,86,98,104,124,134-136,140-143,163-166$

Bildung 29-30, 34

Biochemie 61

Biologie 39, 91, 156, 187-188

Botanik 36-39, 101-102, 115, 163

brute facts; rohe Tatsachen 93, 100, 173 
C
Cartesianismus 58, 103-104
Ceteris-Paribus-Gesetze 63, 65
Chemie 30, 39, 60, 66, 92, 142, 172, 175
Computer; Computermodell 61-62, 188

D

Darstellungstechnik; Darstellungsmittel; Darstellungsform 50, 121, 132, 176, 179

Deduktion; deduktiv; Ableitung 20, 25, 27, 40, 45, 54, 64, 141-145, 162

Deduktiv-nomologisches (DN-) Modell 24

Definition 45-46, 49, 96, 181

Detail 29, 60, 74-76, 79, 92, 99-100, 164

deuten; Deutung 31, 71, 151, 175

Diagramm 50, 122, 124, 153, 174

Dialektik 19, 33, 87

Divination 35,70

\section{E}

einfühlen; Einfühlung 27, 70, 76, 78

Einsicht; nous 22, 26, 28, 36, 48, 56, 133

Empirie 38, 50, 52, 56-57, 64-69, 94-98, 104, 119-124, 137, 162, 192

entdecken; Entdeckung 25, 36, 50, 63, 142, 150, 163, 168, 177, 178, 191

Entwicklungsgeschichte 9, 28, 37

Episteme; scientia 17-18, 21-26, 31-36, 40, 45, 54-57, 62-63, 69, 74, 87, 133-134, 138, 167

Erfahrenheit 56, 95-96

Erfahrung; empeiría 23, 26, 35, 48, 52-56, 60-67, 83, 88, 92-96, 124, 133-137, 148, 151, 153, 162-163

erinnern, Erinnerung 26, 56, 96

erkennen; Erkenntnis 14, 18, 22-25, 31-32, 36, 42, 51-59, 72, 75-77, 80-83, 87-96, $114-116,134-139,153-159,164,167,172,176,181-183,189-194,202$

Erkenntnistheorie 69, 72-73, 80, 84, 88, 90-91, 105, 134, 154-157

erklären; Erklärung 24-27, 30, 34-36, 70-71, 74, 92, 144, 149, 168, 175-177

Ethnologie 78-79, 107, 176

Evidenz 83, 25, 57, 166

Evolution 9, 37, 104-106, 154

Exaktheit 27, 41, 96, 112, 142, 


\section{Expedition 36-38}

experimentieren; Experiment 26, 40, 52-63, 67, 95, 119, 129, 137

\section{$\mathbf{F}$}

Fakten 10, 36, 39, 40, 93-95, 100

Fakultät; facultas 21, 139-140

Fertigkeit 18, 58, 139, 151

Folgerungstechnik 50-51, 62-63, 68-69, 104, 162

formal; Formalismus; symbolischer F.; mathematischer F. 35, 49-51, 54-56, 95, $137,148,151,163,166,172,177-178,189,192,202$

Formel; Formelschreibweise 66, 172

forschen; Forschung; research 9-11, 35, 51, 61-63, 69, 74-75, 79, 89, 124, 127-128$132,137,143,151,164,169,172,175-177,180-194,197,201-202$

G

Galileisches Paradigma 51, 58, 64, 75, 103-105, 163

Ganze, das; katholou 37, 40, 56, 70, 76, 90, 118, 133, 172-175, 197, 202

Geisteswissenschaften 26-27, 30, 69-70, 139, 145-147

Geographie 36, 92, 183, 195

Geometrie; more geometrico 18, 41-50, 64, 72, 135, 140-142, 162

Geowissenschaften 30

Geschichtswissenschaften 69-70,75, 101-102, 110, 143, 146, 164, 168

Geschicklichkeit 48, 53

Gewissheit 84-87

$\mathbf{H}$

Heilige Schrift 34,-35, 74

Hermeneutik 31-35, 69-80, 89, 99, 131-132, 137, 149-150, 162-164, 167

Historia 37

HO-Schema; Hempel-Oppenheim-Schema 24

humanities 29

\section{I}

ideographisch 28, 146

Individuelles 28, 75-79, 85, 113-114, 145, 164, 203

Indiz 71, 75, 164, 174-175

Indizienparadigma 75, 163 
Induktion; inductio; epagogé 10, 40, 48, 52-57, 94, 136, 141-144, 163

Instrument 48, 58, 124, 128-129, 153

Interdisziplinarität 10-11, 14-15, 171-205

Interpretation 31-35, 40, 71, 75-79, 84, 89, 92-94, 98, 129, 134, 143, 150-151, 176, 192

Intuition $25,26,35,70$

J

Juristik; Jurisprudenz 31-33, 139,

Juristische Hermeneutik 33

K

Kausalität 116-117, 123, 127-128, 166, 179

Keplersche Gesetze 64, 66

klassifizieren; Klassifikation 10, 36, 39, 102-103, 106, 110-111, 115, 131, 167

Klimaveränderung 62, 112, 175, 183, 185

Klugheit; phronesis 18, 23, 87, 96, 110, 133

Könnerschaft; Kunst; techné, ars 18, 22, 32-33, 60, 71, 74, 87, 115, 133, 139, 152

Konstruktion 45, 48-49, 73, 104-106, 181

Konstruktivismus 30, 105, 109, 132, 153-162

Kosmologie 36, 74, 108, 154

Krankheit 39, 76, 175

Kriminalistik 69, 99

Kritik 40, 74, 82, 85-94, 107, 111, 131, 137, 140, 143, 153-155, 160-161, 172, 176, 178

$\mathbf{L}$

Labor; Laborwissenschaft 60-62, 65, 69, 176-179

Laborbedingungen 60-62, 147

Logifizierung 56-57

Logik 26, 45-47, 57, 69, 88, 95, 99, 134-135, 141-142, 148-150, 156

Logischer Empirismus (logisch-empiristisch) 147-148

Logos 23

M

Material; Materialität 54-55, 62, 95, 98, 101, 131, 157

Mathematik 26, 30, 33, 41-52, 62, 95, 104-105, 111-113, 127, 133, 139-140, 159, 186189, 192

Mechanik 36, 54, 96-98, 104, 127, 136, 140, 145 
Metaphysik; metaphysisch 52, 97, 105, 115, 138-139, 177

Methode; Methodik 13, 20, 27, 32-33, 39-41, 49, 53-54, 69-72, 85-88, 91, 94, 109$113,137,142-146,157-160,180-190,201-202$

modellieren; Modell; Modellierung 50, 60-62, 101, 116, 161, 175, 186-189, 192

Molekularbiologie 61, 91, 101

Musik 18, 54, 133-135

\section{$\mathbf{N}$}

Natur; physis 20, 37-40, 52-53, 60, 64-66, 80, 96, 104-106, 114, 139, 145, 152$154,161,166-168$

Naturgeschichte; histoire naturelle; natural history 37-40, 53, 138, 162,-163, 166

Naturgesetze; natural laws 27, 52, 62-68, 151-153

Naturphilosophie 69

Naturwissenschaften 26-31, 37-38, 50-55, 59, 64, 69, 88, 100-101, 131, 135, 145147, 153-161

Neuzeit 9, 15, 32, 38-39, 45, 56, 64, 72-73, 83, 87-88, 95, 104, 112, 132, 136, 167

Neuer Experimentalismus; new experimentalism 58, 150-157

Newtonsche Dynamik 66

nomothetisch 28, 146

Notwendigkeit 23-25, 45, 65-66, 144, 176, 180

$\mathbf{0}$

Objekt; object 28, 67, 81-85, 105, 185-189

Objektivität; objektiv 79-86, 157, 161, 173

operatives System; operativer Symbolismus 49, 60-61, 66

Optik 50, 64, 135, 162

$\mathbf{P}$

Pandemie 62, 186

phänomenologische Gesetze; phenomenological laws 67

Philologie 31-33, 78, 88-89, 176

Philosophie 19-26, 33, 42-45, 54, 72, 77, 82-87, 92-94, 107, 133-141, 151, 192

Philosophische Hermeneutik 33, 80

Physik 28-30, 51, 66, 96-98, 111, 115-116, 120, 133, 140-149, 161-163

Physikalismus 15, 149, 186

Physiologie 28, 127, 143, 155

Planeten 24,174 
Politik 41, 185

Postmoderne 30, 91, 160

Postulat 45-46, 49, 165

präparieren; Präparat 38, 100-102

Präskription; Vorschrift 45, 48

Praxis; Praktiken 9, 23, 32, 40, 59, 62, 69, 76, 86, 91, 94, 99-102, 109, 119, 131$133,150-154,165,173-183,192-194,201$

Prinzip 23-27, 35, 40-55, 63, 73-74, 97, 133, 136, 142-145, 168

\section{$\mathbf{R}$}

Rationalität 54, 74, 91, 109, 136-138, 154-156, 166

Rätsel; Rätsellösen; puzzle solving 34-36, 71-76, 180

Realismus 132, 153, 157, 162

Realität 48-49, 67, 105, 123, 154, 158, 182, 202

Rechtswissenschaft 69, 102, 134-135

Regelmäßigkeit 40, 65, 111, 114, 118-128, 168

Regelsystem; Regelwerk 14, 48, 103, 106, 132, 151, 177, 187, 192

rekonstruieren; Rekonstruktion 29, 33-40, 69-81, 131-132, 144, 155, 161, 172, 175-178

Relativismus 109, 156-158

Relikt 75, 172-175, 178, 192

repräsentieren; Repräsentation 68-69, 76, 105

reproduzieren; Reproduktion 46, 61-62, 177, 192

Rhetorik 18, 31-33, 135, 192

\section{S}

sammeln; Sammlung 36-39, 112-119, 178

Schöpfergott 64, 73, 83

science war 30,160

Septem Artes 18, 139

simulieren; Simulation 60-62, 175, 179

Sozialwissenschaften; Soziologie 30-33, 58, 85, 90, 101-102, 111-112, 117, 127, 140, 159-161, 175, 183, 189

Sprachwissenschaften; Linguistik 102-104, 107, 134, 177, 187

Spuren 35, 71, 74, 99, 164-165, 174-175, 178, 192

Statistik 40, 107-130, 166-168, 174, 178, 192

sub specie aeternitatis 23

Syllogistik 44, 48, 57, 134, 141-142 
symbolische Übersetzung; symbolische Verallgemeinerung 66-69, 95 Symptom 71

Synthese; synthetisch 40, 69-73, 144, 167-168, 187, 192

Systematik 33-39, 102, 133

Systemtheorie 15, 187-189

\section{$\mathbf{T}$}

Tabelle 39, 174

Technik 30, 36, 49, 100

Teil und Ganzes 33, 69, 70, 76-77, 92, 131, 137, 175, 192

Theologie 18-21, 133-134, 139

Theorie 41, 53, 59-60, 66-68, 99, 131, 151, 162, 175, 185-189

Typ; Typus 28, 62, 102, 169-176, 183-184, 189-194, 197, 201-202

$\mathbf{U}$

übersetzen; Übersetzung 66-69, 79, 95, 149, 176, 180, 182, 184, 186, 189

Umstände 50, 65-67, 90, 96, 116-118, 143, 160

Unity of Sciene; Einheitswissenschaft 15, 147-149, 186

Universität 15-21, 25, 29-31, 139

\section{V}

variieren; Variation 60, 67, 76, 172-174, 176-178

Verstädterung 62

Verstehen 26-27, 30-37, 69-80, 99, 109, 144, 154

Verstehende Soziologie 33

Verstehenslehre 33-40, 69, 74, 88, 137-138

Vorhersage; prediction 61-63, 128

\section{W}

Weisheit; sophía, sapientia 20-22, 54, 133

Wissenschaftsphilosophie 14, 22-26, 52, 55-58, 62, 69, 149, 160, 177

Wissenschaftssoziologie 14, 151, 158-161, 172

Wissenschaftstheorie (pluralistische) 9, 14

\section{$\mathbf{Z}$}

Zoologie 36-37, 102, 163

zuordnen; Zuordnung 36, 104, 174, 178 


\section{Soziologie}
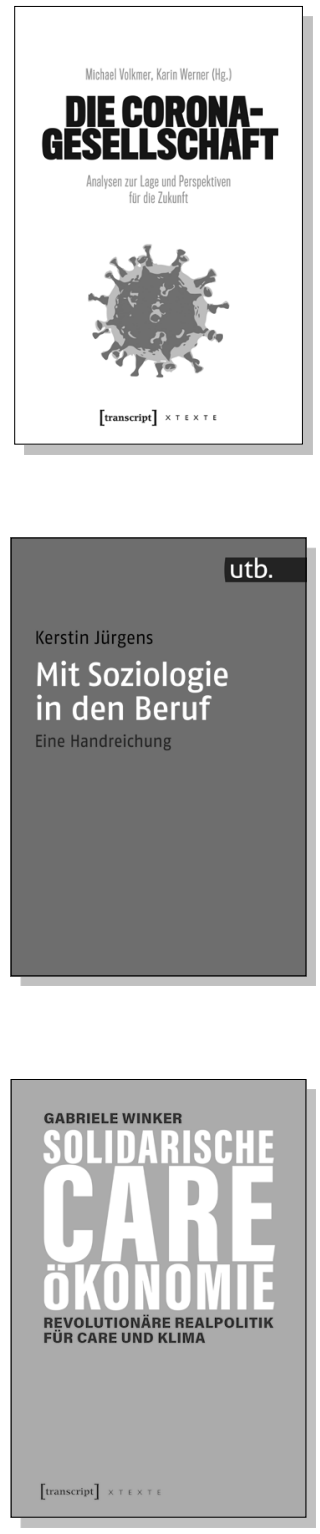

Michael Volkmer, Karin Werner (Hg.)

Die Corona-Gesellschaft

Analysen zur Lage und Perspektiven für die Zukunft

2020, 432 S., kart., Dispersionsbindung, 2 SW-Abbildungen

$24,50 €(D E), 978-3-8376-5432-5$

E-Book:

PDF: 21,99 € (DE), ISBN 978-3-8394-5432-9

EPUB: $21,99 €$ (DE), ISBN 978-3-7328-5432-5

Kerstin Jürgens

Mit Soziologie in den Beruf

Eine Handreichung

September 2021, 160 S., kart., Dispersionsbindung $18,00 €(D E), 978-3-8376-5934-4$

E-Book:

PDF: $15,99 €$ (DE), ISBN 978-3-8394-5934-8

Gabriele Winker

\section{Solidarische Care-Ökonomie}

Revolutionäre Realpolitik für Care und Klima

März 2021, 216 S., kart.

$15,00 €(D E), 978-3-8376-5463-9$

E-Book:

PDF: 12,99€ (DE), ISBN 978-3-8394-5463-3 


\section{Soziologie}

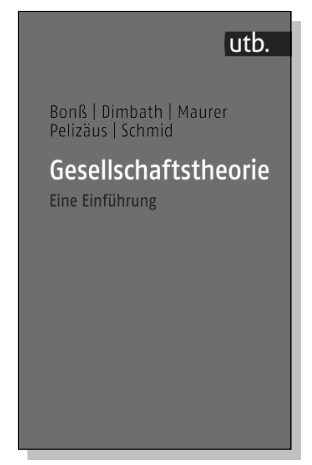

Wolfgang Bonß, Oliver Dimbath,

Andrea Maurer, Helga Pelizäus, Michael Schmid

\section{Gesellschaftstheorie}

Eine Einführung

Januar 2021, 344 S., kart.

25,00€ (DE), 978-3-8376-4028-1

E-Book:

PDF: $21,99 €$ (DE), ISBN 978-3-8394-4028-5

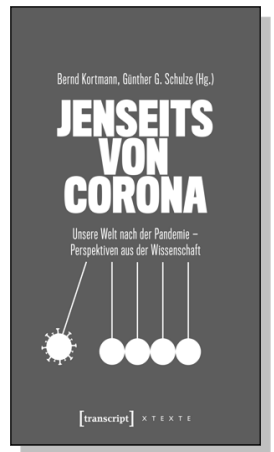

Bernd Kortmann, Günther G. Schulze (Hg.)

\section{Jenseits von Corona}

Unsere Welt nach der Pandemie -

Perspektiven aus der Wissenschaft

2020, 320 S.,

Klappbroschur, Dispersionsbindung, 1 SW-Abbildung

22,50€ (DE), 978-3-8376-5517-9

E-Book:

PDF: $19,99 €$ (DE), ISBN 978-3-8394-5517-3

EPUB: $19,99 €(D E)$, ISBN 978-3-7328-5517-9

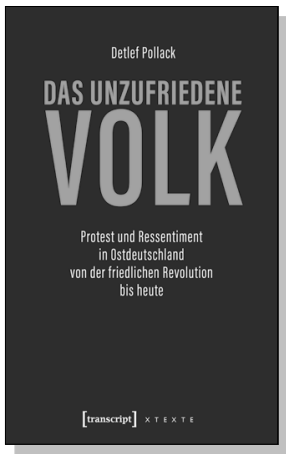

Detlef Pollack

\section{Das unzufriedene Volk}

Protest und Ressentiment in 0stdeutschland von der friedlichen Revolution bis heute

2020, 232 S.,

Klappbroschur, Dispersionsbindung, 6 SW-Abbildungen 20,00€ (DE), 978-3-8376-5238-3

E-Book:

PDF: $17,99 €$ (DE), ISBN 978-3-8394-5238-7

EPUB: $17,99 €(D E)$, ISBN 978-3-7328-5238-3 
